\title{
Hydrologic, Water-Quality, and Meteorological Data for the Cambridge, Massachusetts, Drinking-Water Source Area, Water Year 2005
}

By Kirk P. Smith

Prepared in cooperation with the

City of Cambridge, Massachusetts, Water Department

Open-File Report 2007-1049 


\section{U.S. Department of the Interior DIRK KEMPTHORNE, Secretary}

\section{U.S. Geological Survey \\ Mark D. Myers, Director}

\section{U.S. Geological Survey, Reston, Virginia: 2007}

For product and ordering information:

World Wide Web: http://www.usgs.gov/pubprod

Telephone: 1-888-ASK-USGS

For more information on the USGS — the Federal source for science about the Earth, its natural and living resources, natural hazards, and the environment:

World Wide Web: http://www.usgs.gov

Telephone: 1-888-ASK-USGS

Any use of trade, product, or firm names is for descriptive purposes only and does not imply endorsement by the U.S. Government.

Although this report is in the public domain, permission must be secured from the individual copyright owners to reproduce any copyrighted materials contained within this report.

Suggested citation:

Smith, K.P., 2007, Hydrologic, water-quality, and meteorological data for the Cambridge, Massachusetts, drinkingwater source area, water year 2005: U.S. Geological Survey Open-File Report 2007-1049, 119 p. 


\section{Contents}

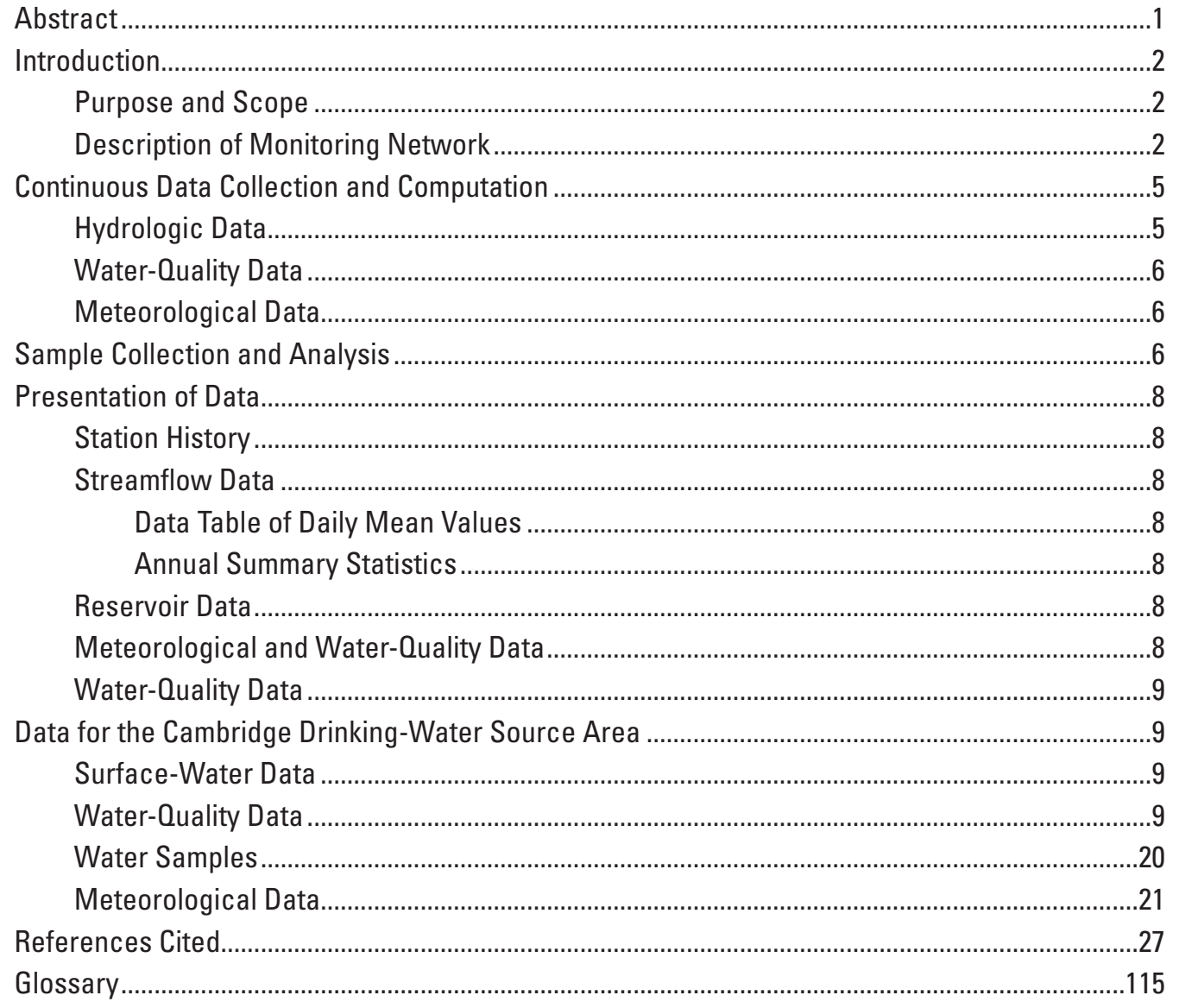

\section{Figures}

1. Map showing the monitoring network for the Cambridge, Massachusetts, drinking-water source area for water year 2005, eastern Massachusetts

2-10. Graphs showing:

2. The percentage of water supplied from the Hobbs Brook basin relative to the total water entering the Stony Brook Reservoir compared to the percentage of water discharged from the Stony Brook Reservoir to the Charles River relative to the total inflow for water year 2005, eastern Massachusetts.

3. Monthly mean reservoir-storage capacities for water year 2005 shown as percent capacity for the Cambridge Reservoir, Stony Brook Reservoir, and Fresh Pond Reservoir, eastern Massachusetts.

4. Monthly mean specific conductance for water year 2005 for USGS station 01104430, Hobbs Brook below Cambridge Reservoir near Kendal Green, Massachusetts, compared to the period-of-record maximum and minimum monthly mean specific conductance, and the median monthly specific conductance for water years 1997-2004. 
5. Monthly mean specific conductance for water year 2005 for USGS station 01104460, Stony Brook at Route 20 at Waltham, Massachusetts, compared to the period-of-record maximum and minimum monthly mean specific conductance, and the median monthly specific conductance for water years 1997-1998 and 2002-2004.

6. Daily mean specific-conductance values for USGS station 422302071083801 , Fresh Pond Reservoir at Cambridge, Massachusetts, for water year 2005

7. (A) Daily total flows greater than 0.01 million gallons per day, and $(B)$ daily maximum and daily minimum specific conductance values for USGS station 01104415, Cambridge Reservoir, unnamed tributary 2, near Lexington, Massachusetts, for water year 2005

8. Monthly mean specific conductance for water year 2005 for USGS station 01104455, Stony Brook, unnamed tributary 1, near Waltham, Massachusetts, compared to the period-of-record maximum and minimum monthly mean specific conductance, and the median monthly specific conductance for water years $1998-2004$

9. Monthly precipitation totals for the Cambridge Reservoir, Stony Brook Reservoir, and Fresh Pond Reservoir in the Cambridge, Massachusetts, drinking-water source area for water year 2005.

10. Monthly mean air temperatures for water year 2005 for the Cambridge Reservoir, compared to the period-of-record maximum and minimum monthly mean air temperatures and the median monthly air temperatures for water years 2002-2004, eastern Massachusetts

\section{Tables}

1. Names, locations, drainage areas, and periods of record for U.S. Geological Survey monitoring stations in the drinking-water source area for Cambridge, Massachusetts, for water year 2005

2. Hydrological, water-quality, and meteorological parameters measured at each continuous-monitoring station during water year 2005, eastern Massachusetts.............5

3. Rating classifications for continuous records of hydrologic, water quality, and meteorological parameters.

4. Extreme measurements of physical parameters for U.S. Geological Survey stations in the drinking-water source area for Cambridge, Massachusetts.

5. Extreme and median constituent concentrations measured in water samples for U.S. Geological Survey monitoring stations in the drinking-water source area for Cambridge, Massachusetts, for the period of record

6. Characteristics of selected pesticides detected in base-flow and stormflow water samples collected in the Hobbs Brook and Stony Brook Reservoir basins, eastern Massachusetts, for water year 2005

7. Frequency of detection and maximum concentration of selected pesticides detected in base-flow and stormflow water samples collected in the Hobbs Brook and Stony Brook Reservoir basins, eastern Massachusetts, for water year 2005

8. Daily, monthly, and annual statistics for discharge for U.S. Geological Survey stations in the drinking-water source area for Cambridge, Massachusetts, for water year 2005 
9. Daily, monthly, and annual statistics for reservoir altitude for U.S. Geological Survey station numbers 01104430, Cambridge Reservoir near Kendal Green, 01104480, Stony Brook Reservoir in Waltham, and 422302071083801, Fresh Pond Reservoir at Cambridge, Massachusetts, for water year 2005.

10. Daily, monthly, and annual statistics for reservoir capacity for U.S. Geological Survey stations 01104430, Hobbs Brook below Cambridge Reservoir near Kendal Green; 01104480, Stony Brook Reservoir in Waltham; and 422302071083801, Fresh Pond Reservoir at Cambridge, Massachusetts, for water year 2005...

11. Daily, monthly, and annual statistics for precipitation for U.S. Geological Survey station numbers 01104430, Cambridge Reservoir near Kendal Green, 01104480, Stony Brook Reservoir in Waltham, and 422302071083801, Fresh Pond Reservoir at Cambridge, water year 2005

12. Daily, monthly, and annual statistics for air temperature for U.S. Geological Survey stations 01104430, Cambridge Reservoir near Kendal Green; 01104480, Stony Brook Reservoir in Waltham; and 422302071083801, Fresh Pond Reservoir at Cambridge, Massachusetts, for water year 2005.

13. Daily, monthly, and annual statistics for water temperature for U.S. Geological Survey stations in the drinking-water source area for Cambridge, Massachusetts, for water year 2005.

14. Daily, monthly, and annual statistics for specific conductance for U.S. Geological Survey stations in the drinking-water source area for Cambridge, Massachusetts, for water year 2005.

15. Physical properties and concentrations of major inorganic constituents, nutrients, trace metals, suspended sediments, Escherichia coli bacteria, polyaromatic hydrocarbons, and polar pesticides and metabolites for base-flow and stormflow water samples collected in four subbasins and the Fresh Pond intake structure in the Cambridge, Massachusetts, drinking-water source area for water year 2005 ....99

16. Concentrations of Escherichia coli for water samples collected during base flow and storms in four subbasins in the Cambridge, Massachusetts, drinking-water source area for water year 2005 . 


\section{Conversion Factors, Datum, and Abbreviations}

\begin{tabular}{lcl}
\hline & Multiply & \multicolumn{1}{c}{ To obtain } \\
\hline inch (in.) & Length & \\
inch (in.) & 2.54 & centimeter $(\mathrm{cm})$ \\
inch (in.) & 25,400 & micron $(\mu \mathrm{m})$ \\
foot (ft) & $25,400,000$ & nanometer $(\mathrm{nm})$ \\
mile (mi) & 0.3048 & meter $(\mathrm{m})$ \\
& 1.609 & kilometer $(\mathrm{km})$ \\
\hline square mile $\left(\mathrm{mi}^{2}\right)$ & Area & \\
\hline & 2.590 & square kilometer $\left(\mathrm{km}^{2}\right)$ \\
\hline million gallons $(\mathrm{Mgal})$ & Volume & \\
\hline & 3785.4 & cubic meter $\left(\mathrm{m}^{3}\right)$ \\
\hline million gallons per day $(\mathrm{Mgal} / \mathrm{d})$ & Flow rate & \\
\hline
\end{tabular}

Temperature in degrees Celsius $\left({ }^{\circ} \mathrm{C}\right)$ may be converted to degrees Fahrenheit ( $\left.{ }^{\circ} \mathrm{F}\right)$ as follows:

$$
{ }^{\circ} \mathrm{F}=\left(1.8 \times^{\circ} \mathrm{C}\right)+32
$$

Vertical coordinate information is referenced to the National Geodetic Vertical Datum of 1929 (NGVD 29).

Horizontal coordinate information is referenced to the North American Datum of 1983 (NAD 83).

Water- and sediment-quality constituents are expressed in milligrams per liter (mg/L), micrograms per liter $(\mu \mathrm{g} / \mathrm{L})$, parts per million (ppm), and parts per billion (ppb). Milligrams per liter is a unit expressing the concentration of chemical constituents in solution as weight (milligrams) of solute per unit volume (liter) of water. One thousand milligrams per liter is equivalent to one gram per liter. One thousand micrograms per liter is equivalent to one milligram per liter. For concentrations less than $7,000 \mathrm{mg} / \mathrm{L}$, the numerical value is the same as for concentrations in ppm.

Specific conductance of water is expressed in microsiemens per centimeter at 25 degrees Celsius $\left(\mu \mathrm{S} / \mathrm{cm}\right.$ at $\left.25^{\circ} \mathrm{C}\right)$.

\section{ABBREVIATIONS}

ASTM American Society for Testing and Materials

COV coefficient of variation

CWD City of Cambridge, Massachusetts, Water Department

EMC event mean concentration

ISO International Organization for Standardization

NIST National Institute of Standards and Technology

PAH polyaromatic hydrocarbons

USEPA U.S. Environmental Protection Agency

USGS U.S. Geological Survey

WY water year 


\title{
Hydrologic, Water-Quality, and Meteorological Data for the Cambridge, Massachusetts, Drinking-Water Source Area, Water Year 2005
}

\author{
By Kirk P. Smith
}

\section{Abstract}

Records of water quantity, water quality, and meteorological parameters were continuously collected from three reservoirs, two primary streams, and four subbasin tributaries in the Cambridge, Massachusetts, drinking-water source area during water year 2005 (October 2004 through September 2005). Water samples were collected during base-flow conditions and storms in the subbasins of the Cambridge Reservoir and Stony Brook Reservoir drainage areas and analyzed for selected elements, organic constituents, suspended sediment, and Escherichia coli bacteria. These data were collected to assist watershed administrators in managing the drinkingwater source area and to identify potential sources of contaminants and trends in contaminant loading to the water supply.

Monthly reservoir capacities for the Cambridge Reservoir varied from about 59 to 98 percent during water year 2005, while monthly reservoir capacities for the Stony Brook Reservoir and the Fresh Pond Reservoir were maintained at capacities greater than 84 and 96 percent, respectively. Assuming a water demand of 15 million gallons per day by the city of Cambridge, the volume of water released from the Stony Brook Reservoir to the Charles River during the 2005 water year is equivalent to an annual water surplus of about 119 percent. Recorded precipitation in the source area for the 2005 water year was within 2 inches of the total annual precipitation for the previous 2 water years.

The monthly mean specific conductances for the outflow of the Cambridge Reservoir were similar to historical monthly mean values. However, monthly mean specific conductances for Stony Brook near Route 20, in Waltham (U.S. Geological Survey station 01104460), which is the principal tributary feeding the Stony Brook Reservoir, were generally higher than the medians of the monthly mean specific conductances for the period of record. Similarly, monthly mean specific conductances for a small tributary to Stony Brook (U.S. Geological Survey station 01104455) were generally higher than the medians of the monthly mean specific conductances for the period of record. The annual mean specific conductance for Fresh Pond Reservoir increased from 514 microsiemens per centimeter $(\mu \mathrm{S} / \mathrm{cm})$ in the 2004 water year to $553 \mu \mathrm{S} / \mathrm{cm}$ for the 2005 water year.
Water samples were collected from four tributaries during base-flow and stormflow conditions in December 2004, and July, August, and September 2005 and analyzed for suspended sediment, 6 major dissolved ions, total nitrogen, total phosphorus, 8 total metals, 18 polyaromatic hydrocarbons (PAHs), 61 pesticides and metabolites, and Escherichia coli bacteria. Concentrations for most dissolved constituents in samples of stormwater were generally lower than the concentrations observed in samples collected during base flow; however, concentrations of total phosphorus, PAHs, suspended sediment, and some total recoverable metals were substantially greater in stormwater samples.

Concentrations of dissolved chloride and total recoverable manganese in water samples collected during base-flow conditions from three tributaries exceeded the U.S. Environmental Protection Agency (USEPA) secondary drinking water standards of 250 and 0.05 milligrams per liter $(\mathrm{mg} / \mathrm{L})$, respectively. Concentrations of total recoverable manganese exceeded the secondary drinking water standard in samples of stormwater from each tributary. Concentrations of total recoverable iron in water samples exceeded the USEPA secondary drinking water standard of $0.3 \mathrm{mg} / \mathrm{L}$ periodically in water samples collected at USGS stations 01104415, 01104455, and 01104475, and consistently in all water samples collected at USGS station 01104433.

Concentrations of Escherichia coli bacteria in water samples collected during base flow ranged from 4 to 1,400 colony-forming units per 100 milliliters (col/100mL). Concentrations of Escherichia coli bacteria in composite samples of stormwater ranged between 1,700 to $43,000 \mathrm{col} / 100 \mathrm{~mL}$ with the highest concentrations measured at USGS station 01104475.

Fluoranthene and pyrene were the most commonly detected PAHs in water samples collected during base flow. Concentrations of PAH compounds observed in composite samples of stormwater were often as much as an order of magnitude or more than concentrations measured in water samples collected during base flow. Fluoranthene, phenanthrene, and pyrene were the only PAH compounds found in a water sample collected from the Fresh Pond Reservoir intake structure. Concentrations of 16 pesticides and caffeine were measured in water samples collected in four subbasins and in Fresh Pond 
Reservoir. Caffeine, imidacloprid, and siduron were the most frequently detected compounds. Each of these compounds also was detected in water collected from the Cambridge water-treatment facility raw-water intake at the Fresh Pond Reservoir. Compounds including 3-ketocarbofuran, carbaryl, MCPA, propoxur, siduron, and triclopyr were only detected in water samples of stormwater.

\section{Introduction}

Hydrologic and water-quality monitoring is important for the effective management and protection of drinking-water supplies. Both the quantity and quality of water are monitored because these factors determine the physical, chemical, and biological state of the water supply. Without accurate information on the past and current condition of the water supply, effective preservation and remediation programs cannot be implemented or evaluated.

The U.S. Geological Survey (USGS) works closely with municipal water suppliers throughout the nation to address specific water problems by conducting hydrologic- and water-quality-monitoring programs and detailed investigations (Patterson, 1997). One such program, conducted from 1997 through 1998 by the USGS in cooperation with the City of Cambridge, Massachusetts, Water Department (CWD), was designed to identify sources of contaminants in the drinkingwater source area for the city (Waldron and Bent, 2001). Subsequently the USGS, in cooperation with the CWD, designed and implemented a water-monitoring network in the drinking-water source area. Data from this network has been published annually in various USGS reports (Smith, 2005; Socolow and others, 1999, 2000, 2001, 2002, 2003, and 2004).

The CWD supplies approximately 15 millions of gallons per $\operatorname{day}^{1}(\mathrm{Mgal} / \mathrm{d})$ to more than 100,000 customers. Most of this water is obtained from three primary storage reservoirs (Cambridge Reservoir-also known as the Hobbs Brook Reservoir, Stony Brook Reservoir, and Fresh Pond Reservoir), in parts of Lexington, Lincoln, Waltham, Weston, and in Cambridge (fig. 1). The drainage basin for the Cambridge Reservoir includes Hobbs Brook and three unnamed tributaries that discharge directly into the reservoir. Water is discharged from the southern end of the Cambridge Reservoir into Hobbs Brook which receives additional water by an unnamed tributary about a half mile below the reservoir. Hobbs Brook joins with Stony Brook about 1.6 miles downstream of the reservoir and flows south to the Stony Brook Reservoir. Two unnamed tributaries flow into Stony Brook about a quarter mile north of the Stony Brook Reservoir. In addition to water from Stony Brook, an unnamed tributary flows directly into the Stony Brook Reservoir on the southwest side of the reservoir. Additional water enters both reservoirs from other minor tributaries and highway and parking-lot storm drains. Water from Stony
Brook Reservoir is piped through an aqueduct by the CWD directly to Fresh Pond Reservoir where it is stored prior to treatment. Overflow and controlled discharges from the Stony Brook Reservoir flow into the Charles River in Waltham.

The drainage basin contributing water to these reservoirs has undergone rapid development in recent years and encompasses major transportation corridors, as well as large areas of industrial, commercial, and high-density residential land use. Because the City of Cambridge owns less than 5 percent of the land in the basin contributing to its water-supply system, the CWD relies heavily on monitoring to provide information for optimizing the management of its reservoirs for water quality and quantity. The USGS monitoring network provides near-real-time information that assists the CWD in responding rapidly to water-quality changes caused by accidental or intentional contamination. This information also benefits the CWD, other municipalities, and state agencies involved with water-resource development and management in the Charles River Basin by enhancing their understanding of the relation between local drinkingwater-management practices and regional issues of water supply and hydrologic-system response.

\section{Purpose and Scope}

This report presents records of water quantity, water quality, and meteorological parameters collected in the Cambridge, Massachusetts, drinking-water source area during water year 2005 (October 2004 through September 2005). It describes the monitoring network, data-collection methods for all types of data, and computation methods. It also describes the chemical characteristics of water samples collected during base-flow conditions and during storms from an unnamed tributary in the Cambridge Reservoir basin and three unnamed tributaries in the Stony Brook Basin, and of water samples collected from the Cambridge water-treatment facility.

\section{Description of Monitoring Network}

Stations installed and operated by the USGS in the drinking-water source area continuously monitored various hydrologic, water-quality, and meteorological parameters including stream stage, stream water temperature, stream specific conductance, reservoir altitude, air temperature, and precipitation. Stations were selected for continuous monitoring on the basis of the necessity for water-supply regulation by the CWD and of information gained in previous USGS investigations that identified specific areas as potentially important sources of contaminants. Attributes of the monitoring stations are listed in table 1; locations of stations selected for continuous monitoring are shown in figure 1.

\footnotetext{
${ }^{1}$ Terms listed in the glossary at the back of this report are in bold type where first used in the text.
} 

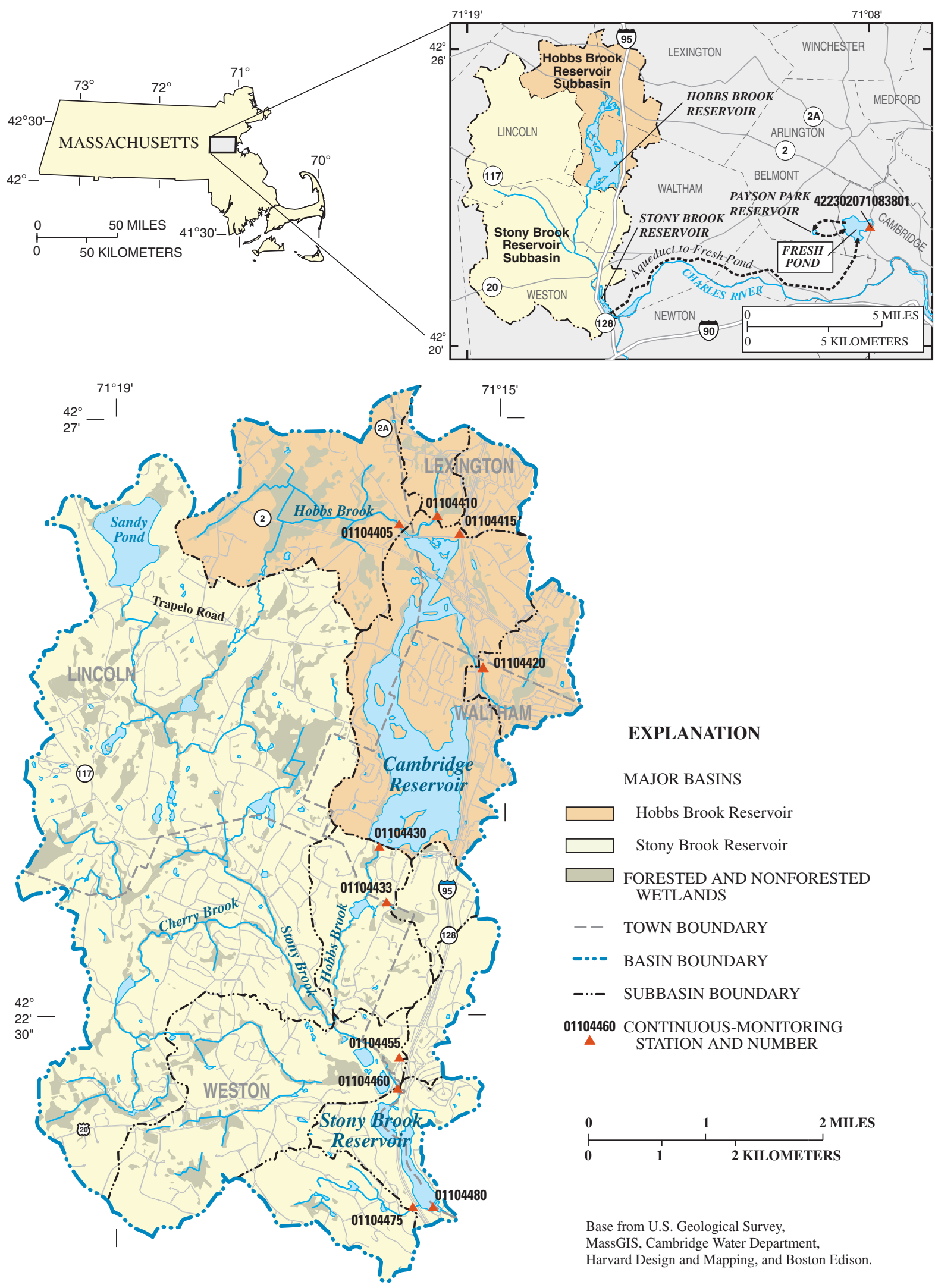

Figure 1. The monitoring network for the Cambridge, Massachusetts, drinking-water source area for water year 2005, eastern Massachusetts. 
Table 1. Names, locations, drainage areas, and periods of record for U.S. Geological Survey monitoring stations in the drinkingwater source area for Cambridge, Massachusetts, for water year 2005.

[USGS, U.S. Geological Survey. ํ, degree; ', minute; ", second; mi², square mile]

\begin{tabular}{|c|c|c|c|c|c|}
\hline Station name & $\begin{array}{l}\text { USGS station } \\
\text { number }\end{array}$ & Latitude & Longitude & $\begin{array}{l}\text { Drainage } \\
\text { area }\left(\mathrm{mi}^{2}\right)\end{array}$ & Period of record \\
\hline Stony Brook at Kendal Green & 01104390 & $42^{\circ} 22^{\prime} 36^{\prime \prime}$ & $71^{\circ} 16^{\prime} 55^{\prime \prime}$ & 10.4 & 03/07/97-09/17/98 \\
\hline $\begin{array}{l}\text { Cambridge Reservoir, unnamed tributary } 1 \text {, } \\
\text { near Lexington }\end{array}$ & 01104410 & $42^{\circ} 26^{\prime} 15^{\prime \prime}$ & $71^{\circ} 15^{\prime} 53^{\prime \prime}$ & 2.1 & 03/05/97-09/16/98 \\
\hline $\begin{array}{l}\text { Cambridge Reservoir, unnamed tributary } 3 \text {, } \\
\text { near Lexington }\end{array}$ & 01104420 & $42^{\circ} 25^{\prime} 11^{\prime \prime}$ & $71^{\circ} 15^{\prime} 29^{\prime \prime}$ & .73 & 04/09/97-09/16/98 \\
\hline $\begin{array}{l}\text { Hobbs Brook below Cambridge Reservoir } \\
\text { near Kendal Green }\end{array}$ & 01104430 & $42^{\circ} 23^{\prime} 53^{\prime \prime}$ & $71^{\circ} 16^{\prime} 26^{\prime \prime}$ & 6.86 & 04/09/97-09/16/98 \\
\hline Stony Brook at Route 20 near Waltham & 01104460 & $42^{\circ} 21^{\prime} 08^{\prime \prime}$ & $71^{\circ} 16^{\prime} 16^{\prime \prime}$ & 22 & $\begin{array}{l}\text { 03/07/97-10/28/98; } \\
\text { 05/14/02-current year }\end{array}$ \\
\hline $\begin{array}{l}\text { Stony Brook Reservoir, unnamed tributary } 1 \\
\text { near Weston }\end{array}$ & 01104475 & $42^{\circ} 21^{\prime} 16^{\prime \prime}$ & $71^{\circ} 16^{\prime} 07^{\prime \prime}$ & .85 & $\begin{array}{l}\text { 12/17/97-09/17/98; } \\
\text { 08/26/04-current year }\end{array}$ \\
\hline Stony Brook Reservoir at dam near Waltham & 01104480 & $42^{\circ} 21^{\prime} 20^{\prime \prime}$ & $71^{\circ} 15^{\prime} 56^{\prime \prime}$ & 23.7 & 03/1997-current year \\
\hline Fresh Pond in gate house at Cambridge & 422302071083801 & $42^{\circ} 23^{\prime} 02^{\prime \prime}$ & $71^{\circ} 08^{\prime} 38^{\prime \prime}$ & 0 & 1998; 10/01/03-current year \\
\hline
\end{tabular}

Stream-stage measurements were recorded at monitoring stations at the outlet of the Cambridge Reservoir, on Stony Brook, and at the outlet of Stony Brook Reservoir (USGS stations 01104430, 01104460 and 01104480). Water-quality measurements at the outlet of the Cambridge Reservoir and Stony Brook were recorded at USGS stations 01104430 and 01104460. Physical parameters monitored at these sites are listed in table 2 . These data were recorded at a frequency of 15 minutes and were uploaded to a USGS database on an hourly basis by phone modem. In addition to measurements made on these streams, stream-stage measurements and waterquality measurements were recorded at monitoring stations on four of the small tributaries (USGS stations 01104415, 01104433, 01104455, and 01104475). Because the drainage areas of these sites are small (less than a square mile) and contain many roadways, parking lots, and other impervious surfaces (Waldron and Bent, 2001), the hydrologic responses, and often the water-quality responses, change rapidly. To document these responses effectively, the monitoring stations recorded stream-stage and water-quality measurements at variable frequencies, as often as 1 minute. These data were uploaded to a USGS database on an hourly basis by digital cellular modem.

Measurements of reservoir altitude, precipitation, and air temperature were recorded at the Cambridge, Stony Brook, and Fresh Pond Reservoirs (USGS stations 01104430, 01104480, and 422302071083801). Water-quality measurements of reservoir water also were recorded at the Fresh Pond Reservoir. Physical parameters monitored at these sites are listed in table 2. These data were recorded at a frequency of 15 minutes and were uploaded to a USGS database on an hourly basis by phone modem. 
Table 2. Hydrological, water-quality, and meteorological parameters measured at each continuous-monitoring station during water year 2005, eastern Massachusetts.

[USGS, U.S. Geological Survey. X, indicates physical parameter was measured]

\begin{tabular}{lcccccccc}
\hline \multirow{2}{*}{$\begin{array}{c}\text { Physical } \\
\text { parameter }\end{array}$} & \multicolumn{8}{c}{ USGS station number } \\
\cline { 2 - 9 } & $\mathbf{0 1 1 0 4 4 1 5}$ & $\mathbf{0 1 1 0 4 4 3 0}$ & $\mathbf{0 1 1 0 4 4 3 3}$ & $\mathbf{0 1 1 0 4 4 5 5}$ & $\mathbf{0 1 1 0 4 4 6 0}$ & $\mathbf{0 1 1 0 4 4 7 5}$ & $\mathbf{0 1 1 0 4 4 8 0}$ & $\mathbf{4 2 2 3 0 2 0 7 1 0 8 3 8 0 1}$ \\
\hline Stream water stage & $\mathrm{X}$ & $\mathrm{X}$ & $\mathrm{X}$ & $\mathrm{X}$ & $\mathrm{X}$ & $\mathrm{X}$ & $\mathrm{X}$ & \\
Reservoir water level & & $\mathrm{X}$ & & & & & $\mathrm{X}$ & $\mathrm{X}$ \\
Precipitation & & $\mathrm{X}$ & & & & & $\mathrm{X}$ & $\mathrm{X}$ \\
Air temperature & & $\mathrm{X}$ & & & & & $\mathrm{X}$ & $\mathrm{X}$ \\
Water temperature & $\mathrm{X}$ & $\mathrm{X}$ & $\mathrm{X}$ & $\mathrm{X}$ & $\mathrm{X}$ & $\mathrm{X}$ & & $\mathrm{X}$ \\
Specific conductance & $\mathrm{X}$ & $\mathrm{X}$ & $\mathrm{X}$ & $\mathrm{X}$ & $\mathrm{X}$ & $\mathrm{X}$ & & $\mathrm{X}$ \\
\hline
\end{tabular}

\section{Continuous Data Collection and Computation}

The monitoring network provides near-real-time information used to manage the quantity and quality of water in the CWD drinking-water source area. At each station, permanently installed analytical equipment automatically measures selected hydrologic, water-quality, and meteorological parameters and relays the information to water managers through telephone voice modems and through the USGS Massachusetts-Rhode Island Water Science Center on the World Wide Web (http://ma.water.usgs.gov).

\section{Hydrologic Data}

Basic data collected at the monitoring stations include records of stream stage and measurements of discharge of streams, and water altitude and capacity of reservoirs. In addition, observations of factors affecting the stage-discharge relation or the altitude-capacity relation, weather records, and other information are used to supplement the basic data in determining the daily flow or capacity of water in storage. Measurements of discharge are made with a current meter or acoustic Doppler current profiler by standard USGS methods (Buchanan and Somers, 1968, 1969; Carter and Davidian, 1968; Kennedy, 1983 and 1984; Oberg and others, 2005; Rantz and others, 1982). The methods are consistent with the American Society for Testing and Materials (ASTM) standards and generally follow the standards of the International Organization for Standards (ISO).
To determine streamflow at each USGS monitoring station in the CWD drinking-water source area, dischargerating tables for any stage are prepared from stage-discharge curves (Rantz and others, 1982). The daily mean discharge is computed from these stage and rating tables, and then the monthly and yearly mean discharges are computed from these daily values. If the stage-discharge relation for a station is temporarily changed by aquatic growth or debris in the control section, the daily mean discharge is computed by the shifting-control method (Rantz and others, 1982).

For the USGS monitoring stations on reservoirs in the CWD drinking-water source area, capacity tables giving the volume for any reservoir water altitude are prepared from water altitude-area relation curves defined by surveys (Fugro East, Inc., 1996). From the tables, the daily, monthly, or yearly changes in volume are computed.

For some stations, recorder or sensor malfunctions can cause gaps in the water-stage record or inaccurate readings, which cannot be used to compute daily discharge. For periods of malfunction, the daily mean discharges are estimated on the basis of the recorded range in stage, prior and subsequent records, discharge measurements, weather records, and comparison with records from other stations in the same or nearby basins. Likewise, reservoir volumes may be estimated on the basis of operator's log, prior and subsequent records, and other information.

The accuracy of hydrologic data depends primarily on (1) the stability of the stage-discharge relation or, if the control is unstable, the frequency of discharge measurements, and (2) the accuracy of observations of stage, measurements of discharge, and interpretations of records. The degree of accuracy of the records is defined in table 3. Different accuracies may be attributed to different portions of a streamflow record. 
Table 3. Rating classifications for continuous records of hydrologic, water quality, and meteorological parameters.

[Modified from Wagner and others, 2003. $\leq$, less than or equal to; \pm , plus or minus value shown; ${ }^{\circ} \mathrm{C}$, degrees Celsius; $>$, greater than; \%, percent; $\mathrm{ft}$, foot]

\begin{tabular}{|c|c|c|c|c|}
\hline \multirow{2}{*}{$\begin{array}{c}\text { Measured } \\
\text { physical } \\
\text { parameter }\end{array}$} & \multicolumn{4}{|c|}{ Rating } \\
\hline & Excellent & Good & Fair & Poor \\
\hline Discharge & $\leq \pm 5 \%$ & $> \pm 5$ to $10 \%$ & $> \pm 10$ to $15 \%$ & $> \pm 15 \%$ \\
\hline $\begin{array}{l}\text { Reservoir } \\
\text { altitude }\end{array}$ & $\leq \pm 0.1 \mathrm{ft}$ & $> \pm 0.1$ to $0.2 \mathrm{ft}$ & $> \pm 0.2$ to $0.3 \mathrm{ft}$ & $> \pm 0.3 \mathrm{ft}$ \\
\hline $\begin{array}{l}\text { Reservoir } \\
\text { capacity }\end{array}$ & $\leq \pm 1 \%$ & $> \pm 1$ to $2 \%$ & $> \pm 2$ to $4 \%$ & $> \pm 4 \%$ \\
\hline $\begin{array}{l}\text { Water } \\
\text { temperature }\end{array}$ & $\leq \pm 0.2^{\circ} \mathrm{C}$ & $> \pm 0.2$ to $0.5^{\circ} \mathrm{C}$ & $> \pm 0.5$ to $0.8^{\circ} \mathrm{C}$ & $> \pm 0.8^{\circ} \mathrm{C}$ \\
\hline $\begin{array}{l}\text { Specific } \\
\text { conductance }\end{array}$ & $\leq \pm 3 \%$ & $> \pm 3$ to $10 \%$ & $> \pm 10$ to $15 \%$ & $> \pm 15 \%$ \\
\hline Precipitation & $\leq \pm 2 \%$ & $> \pm 2$ to $6 \%$ & $> \pm 6$ to $10 \%$ & $> \pm 10 \%$ \\
\hline $\begin{array}{l}\text { Air } \\
\text { temperature }\end{array}$ & $\leq \pm 0.4^{\circ} \mathrm{C}$ & $> \pm 0.4$ to $0.8^{\circ} \mathrm{C}$ & $> \pm 0.8$ to $2^{\circ} \mathrm{C}$ & $> \pm 2^{\circ} \mathrm{C}$ \\
\hline
\end{tabular}

\section{Water-Quality Data}

Water temperature and specific conductance data were collected from continuous water-quality monitors in each stream, tributary, or reservoir except for USGS station 01104480. The accuracy of the water-quality records depends primarily on the rate of sensor drift, sensor fouling, and debris collection. Typically, sensors became fouled by aquatic growth more rapidly in the warmer months. In most cases, corrections for fouling and drift can be applied to the data to improve their accuracy (Wagner and others, 2003). For parameters other than water temperature, such corrections were made on the basis of the performance of the sensor before and after sensor maintenance and by noting the response of the clean sensor after placing it in several standardized solutions. The accuracy of water temperature data is determined by comparing measurements made by the monitoring system and by an independent probe calibrated against a National Institute of Standards and Technology (NIST) traceable thermometer. One of four accuracy classifications ranging from excellent to poor is applied to physical properties measured at each station. The accuracy rating is based on data values recorded before any shifts or corrections are made for fouling and drift. The basis for each rating classification is listed in table 3 .

\section{Meteorological Data}

Precipitation data were collected with heated tippingbucket precipitation gages that measure the volume of rain or melted snow in 0.01-inch increments. The precipitation gages at the Cambridge Reservoir and Stony Brook Reservoir include wind screens that reduce bias generated by precipitation missing the instrument. Precipitation data are summed for each day and then for each month. In general, the accuracy of precipitation data is assured by proper maintenance and calibration of the device. Precipitation measurements, especially when the precipitation is in the form of snow are affected by strong winds and are subject to errors. These errors generally result in underestimating the total precipitation at a station.

Air temperature data were collected with thermistors housed in gill radiation shields. The probes are installed approximately 8 feet above ground surface. The maximum, minimum, and mean temperature values are computed for each day. Monthly statistics are then computed from daily values. The accuracy of air temperature data is determined by comparing measurements made by the monitoring system and by an independent probe calibrated against a NIST-traceable thermometer.

Under rare circumstances, when there were no records of precipitation or air temperature, daily values were estimated on the basis of records from nearby stations. These circumstances may include a recorder malfunction, the plugging of the precipitation gage, or a malfunction of the heating element in the precipitation gage.

\section{Sample Collection and Analysis}

Water samples were collected during base flow in four tributaries in the Cambridge Reservoir and Stony Brook Reservoir basins in December 2004 and August 2005. Water samples were collected during storms in the same four tributaries in July, August, and September 2005. A sample of raw and finished water also was collected in July at the Cambridge water treatment plant. Most samples were analyzed for physical, chemical, and biological constituents.

Water samples were collected during base-flow conditions and during rain storms from four tributaries near USGS monitoring stations $01104415,01104433,01104455$, and 01104475 and analyzed for distributions of particle size and concentrations of suspended sediment, 6 major dissolved ions, total nitrogen, total phosphorus, 8 total metals, 18 polyaromatic hydrocarbons (PAHs), 61 pesticides and metabolites, and Escherichia coli bacteria. Samples of stream water were collected manually (Wilde, and others, 1999) under base-flow conditions with an antecedent dry period of at least 5 days. During storms, water samples for chemical analysis were collected with an automatic sampler controlled by a datalogger. The first sample was collected when flow exceeded a preset flow threshold, and subsequent samples were collected at flow-proportional intervals. Each automatic 
sampler was configured to hold one 20-liter (L) Teflon-lined plastic bottle and fitted with a pre-cleaned 1/2-in. inner diameter Teflon intake tube.

A multi-step process was used to clean all wetted parts associated with the automatic sampler and the processing equipment before collecting trace inorganic and organic constituents. The initial cleaning consisted of washing the interior and exterior with a phosphate-free laboratory grade soap and tap water, scrubbing surfaces with a plastic brush, and rinsing with tap water. Circulating the solution through the tubing cleaned the interior of the sampler tubing. Lint-free wipes were forced hydraulically through the tubing to remove internal deposits or films that were difficult to remove by circulating solution alone. After the components dried, they were placed in a large stainless steel pan in a fume hood and immersed in a 1-to-1 hexane-to-acetone solution. A Teflon diaphragm pump was used to circulate the solution through the sampler tubing. The components were allowed to soak, with occasional agitation, for a period of about 6 hours. After appropriately dispensing the waste solution, all components except the tubing were rinsed with a 1-to-1 hexane-to-acetone solution from a Teflon squeeze bottle and air-dried in a fume hood over night. Because the rate of cleaning-solution volatilization was limited within the sampler tubing, the tubing was purged with purified air for approximately 20 minutes. The final steps involved immersing the components in a 5-percent solution of hydrochloric acid for a period of at least 6 hours. The same solution was slowly circulated through each sampler tube for six hours or more. All components were thoroughly rinsed with deionized water until the specific conductance of the waste rinse water was less that 1 microsiemens per centimeter $(\mu \mathrm{S} / \mathrm{cm})$.

Samples for chemical analysis were processed in the field, usually at the conclusion of each runoff event. Subsamples for the analysis of suspended sediment, and inorganic and organic constituents were split directly from the Teflon-lined bottle. This method eliminated sample contact with additional processing equipment and reduced the potential for contamination. Subsamples were dispensed under low pressure directly from the sample bottle with a specialized cap, which included a 6.35-millimeter (inner diameter) Teflon dispensing tube, a pressure port, and a relief valve. Compressed nitrogen gas applied to the pressure port filled the interior area between the bottle wall and the Teflon bag, compressing the bag and dispensing the sample. Homogenization of the sample was accomplished by fastening the bottle to a cradle assembly capable of rotating 210 degrees. The sample bottle was rocked the full 210 degrees several times prior to dispensing, and the rocking motion was continued throughout dispensing for all samples except for samples analyzed for dissolved constituents. Dissolved constituents were filtered through a 600 -square-centimeter capsule filter with a 0.45 -micrometer pore size.

Flow-proportional water samples for the analysis of bacteria were collected from a second dedicated automatic sampler. Each automatic sampler was configured to hold between one and four 3.75-L glass bottles. Sample lines consisted of silicon pump-head tubing and polyethylene intake tubing. The sample bottles and pump-head tubing were pre-cleaned and sterilized in an autoclave. New polyethylene intake tubing was checked for sterility prior to installation, used once, and discarded. The sampler base was packed with bagged ice prior to each storm. Samples for analysis of bacteria were collected automatically at the same frequency as samples collected for chemical analysis. Samples for bacteria analysis were processed in the field within 6 hours of the time that the automatic sampler was triggered to collect the first sample for each bottle. When possible, event mean concentrations (EMCs) of Escherichia coli bacteria were mathematically determined by calculating the average values for flow-weighted concentrations of sub-composites.

In addition to the environmental samples, water samples also were collected for quality assurance. These quality-assurance samples include a source-solution blank, a field blank, several processing-equipment blanks, and replicate samples. A source-solution blank was prepared from deionized water produced by a laboratory-grade water-purification system that uses ion-exchange packs and reverse osmosis. The blank water was stored in a Teflon-lined bottle until it was subsequently used for a field blank. A field blank was collected during sample preparation for the July storm. A field blank is used to test for positive bias that can result from contamination from any stage of the sample collection and processing phase or analytical process. The field blank was collected by circulating source water through the automatic sampler tubing and bottle, and processed in a manner consistent with the collection of environmental samples of stormwater. Several processing-equipment blanks were collected for bacteria analysis. A processing-equipment blank is used to test for positive bias that can result from contamination from the ambient environment under which the samples are processed, and from contamination of the filters, filter plate, dilution media, agar, Petri dishes, and other equipment that may be used for the processing of water samples for bacteria analysis. Finally, several replicate-split samples were prepared from samples collected during base flow and storms. Replicate-split samples provide a measure of variability introduced during sample processing and analysis.

Samples were analyzed for concentrations of suspended sediment and distribution of particle size at the USGS Kentucky Water Science Center Sediment Lab (Guy, 1970; Sholar and Shreve, 1998). Samples were analyzed for chemical concentrations at the USGS Laboratory in Denver, Colorado (Patton and Kryskalla, 2003; Furlong and others, 2001; American Public Health Association, 1998; Garbarino and Struzeski, 1998; Hoffman and others, 1996; Fishman, 1993; McLain, 1993; Fishman and Friedman, 1989; Wershaw and others, 1987). Samples were processed for bacteria analysis on the basis of the methods described by Myers and Sylvester (1997) and the U.S. Environmental Protection Agency (USEPA) method 1603 (2002b) on site and placed in portable incubators in a mobile field laboratory. 


\section{Presentation of Data}

Data collected at the USGS monitoring stations during water year 2005 are presented in the back of the report in tables 8-16. Where available, each table includes a summary of daily values, monthly statistics, annual statistics, gage datum, and an accuracy rating for each station. The period of record and the period-of-record extremes for each physical parameter are presented in table 4 by USGS station number. The maximum, median, and minimum statistics for selected analytical results for the period of record for each USGS monitoring station are presented in table 5. Analytical results for water samples are presented in the back of the report in tables 15 and 16 .

\section{Station History}

The station history for each USGS station in the Cambridge drinking-water source area is presented in table 4 . This table includes basic information about the period of record and the extremes for the period of record for each physical parameter for current and historical USGS stations in the Cambridge drinking-water source area. The "period of record" is the time during which each type of data has been collected at the station. These data may have been collected manually on an intermittent basis or continuously with a monitoring system. The "extremes for the period of record" refer to the maximum and minimum values measured during the period of record.

\section{Streamflow Data}

Streamflow data are presented in table 8. This table includes the daily mean values of discharge by USGS station number for the 2005 water year, monthly summary statistics, annual summary statistics and data pertaining to annual runoff and flow duration.

\section{Data Table of Daily Mean Values}

The daily table of streamflow records gives the total discharge for each day of the water year. In the monthly summary for the table, the line labeled "Total" gives the sum of the daily streamflows for each month; the line labeled "Mean" gives the arithmetic average of the daily streamflows for the month; the lines labeled "Maximum" and "Minimum" give the maximum and minimum daily streamflows respectively, for each month; and the line labeled "Median" gives the median daily streamflow for each month. Discharge per unit area of the drainage basin for the month is expressed in millions of gallons per day per square mile (line labeled $\mathrm{Mgal} / \mathrm{d} / \mathrm{mi}^{2}$ ); runoff is given in inches (line labeled "Inches"). Values for discharge per unit area and runoff in inches are not calculated for stations affected by reservoir regulation.

\section{Annual Summary Statistics}

Annual summary statistics are presented at the end of the water year following the daily mean values and monthly summaries. These statistics include the annual total, annual mean, annual runoff, and selected streamflow-duration percentiles. In the annual summary for the table, the line labeled "Annual total" gives the sum of all the daily discharges for the water year; the line labeled "Annual mean" gives the arithmetic average of the daily discharges for the water year. The line labeled "Annual $\mathrm{Mgal} / \mathrm{d} / \mathrm{mi}^{2}$ " is the average number of millions of gallons of water flowing per day from each square mile of area drained; the runoff is assumed to be distributed uniformly in time and area annually. The line labeled "Annual inches" indicates the depth to which the drainage area would be covered if all of the runoff for a given time period were uniformly distributed on it. Runoff data are omitted for stations affected by reservoir regulation. The line labeled "10 percent exceeds" indicates the discharge that has been exceeded 10 percent of the time for the designated period (the 10-percent flow-duration discharge). The lines labeled "50 percent exceeds" and "90 percent exceeds" are similarly defined. The line labeled "remarks" indicates the accuracy of the records (table 2 ).

\section{Reservoir Data}

Continuous records of reservoir altitude and capacity for the Cambridge Reservoir, Stony Brook Reservoir, and the Fresh Pond Reservoir are presented in tables 9 and 10, respectively. Data tables for each parameter consist of daily mean values that include monthly and annual minimum, maximum, and mean values, a gage description, and an accuracy rating description. The line labeled "Gage" indicates the value and source of the datum used to monitor reservoir altitudes. The line labeled "Remarks" indicates the accuracy of the records (table 2). The accuracy of reservoir capacity is determined on the basis of the average annual altitude for each reservoir and the difference in capacity associated with the error in the measurements of the reservoir altitude.

\section{Meteorological and Water-Quality Data}

Continuous records of meteorological and water-quality parameters are presented in tables 11 through 14. Data tables for precipitation consist of daily totals that also include monthly and annual totals and maximum values, and an accuracy rating description. Data tables for all other parameters consist of daily maximum, minimum, and mean values that also include monthly and annual minimum, maximum, and mean values, and an accuracy rating description. 


\section{Water-Quality Data}

Analytical results for water samples, including concentrations of Escherichia coli bacteria in samples of water collected during base-flow conditions and estimated concentrations of Escherichia coli bacteria for composites of stormwater are presented in table 15. Analytical results for concentrations of Escherichia coli bacteria for all samples are presented in table 16. Quality-control samples are listed at bottom of each table. The maximum, median, and minimum statistics for concentrations of selected constituents in water samples collected during the period of record for each USGS station in the Cambridge drinking-water source area are presented in table 5.

\section{Data for the Cambridge Drinking-Water Source Area}

The data presented in this report can be used by the water-resource managers in the city of Cambridge, Massachusetts, to optimize the management of the drinking-water-supply reservoirs for water quality and quantity. The data allow clarification of the relation between reservoir management practices and current conditions, and trends in water quantity and quality in the subbasins of the hydrologic system.

\section{Surface-Water Data}

From October 2004 through May 2005, water from the Hobbs Brook Basin accounted for about 8 to 40 percent (estimated by dividing the sum of monthly discharges measured at USGS stations 01104430 and 01104433 by the sum of monthly discharges measured at USGS stations 01104460 and 01104475) of the water entering the downstream Stony Brook Reservoir (fig. 2). During the remainder of the water year (June through September), the amount of water from the Hobbs Brook Basin, most of which was released from the Cambridge Reservoir, steadily increased to about 99 percent of the total inflow of the Stony Brook Reservoir despite somewhat uniform rainfall during the summer. From December 2004 through May 2005, about 70 percent of all water entering the Stony Brook Reservoir (estimated by dividing the sum of monthly reservoir outflows measured at USGS station 01104480 by the sum of monthly discharges measured at USGS stations 01104460 and 01104475) was diverted to the Charles River (fig. 2). The volume of water released from the Stony Brook Reservoir to the Charles River during water year 2005 represents an annual surplus of about 119 percent of the total annual water demand by the city of Cambridge. During the past 5 years, this surplus has ranged from 29 to 155 percent assuming an average demand of $15 \mathrm{Mgal} / \mathrm{d}$.

Monthly reservoir capacities for the Cambridge Reservoir varied from about 59 to 98 percent during water year 2005 (fig. 3). The reservoir was near capacity in the spring, but was subsequently drawn down as additional water was needed to supplement the city of Cambridge water demand when the yield from the Stony Brook basin diminished. Monthly reservoir capacities for the Stony Brook Reservoir and the Fresh Pond Reservoir were maintained at capacities greater than 84 and 96 percent, respectively, during water year 2005 (fig. 3). In January 2005, the CWD installed three stop logs in the Cambridge Reservoir spillway, consequently increasing the spillway altitude by an additional 1.6 feet and the reservoir capacity by an estimated 220 millions gallons (Mgal) or 8.8 percent.

\section{Water-Quality Data}

The monthly mean specific conductances for water discharged from the Cambridge Reservoir were equal to or within the interquartile range of the monthly mean specific conductances for the period of 1997 through 2005 (fig. 4). The monthly mean specific conductances for Stony Brook at USGS station 01104460 were generally higher than medians of the monthly mean specific conductances for the period of 1997 through 1998 and 2002 through 2004, and for 5 months, new maximum monthly mean specific conductances were calculated (fig. 5). The annual mean specific conductance for Fresh Pond Reservoir increased from $514 \mu \mathrm{S} / \mathrm{cm}$ in the 2004 water year (Smith, 2005) to $553 \mu \mathrm{S} / \mathrm{cm}$ during the 2005 water year (table 14). The running average specific conductance for Fresh Pond Reservoir increased by $43 \mu \mathrm{S} / \mathrm{cm}$ or about 8 percent between July 1 and September 30 (fig. 6) as a result of the increasing influx of water from the Cambridge Reservoir, which has a greater specific conductance, compared to water from the principal stream in the Stony Brook Basin.

Many of the small tributaries to the reservoirs differ from the principal streams in that the water quantity, as well as the water quality of the tributaries, responds more rapidly to stormwater runoff. Data illustrating these effects for flow and specific conductance at USGS station 01104415 are shown in figure 7. The daily mean specific conductance values for USGS stations 01104415, 01104433, and 01104455 differed by as much as 661 percent relative to the annual mean values during water year 2005. Daily mean specific conductance values for USGS station 01104475 differed by about $155 \mu \mathrm{S} / \mathrm{cm}$ or about 55 percent of the mean annual value during water year 2005. The coefficient of variation (COV) for monthly mean specific conductance values ranged from about 8 percent to about 35 percent $(01104475>01104415>01104433>01104$ $455)$. On the basis of 7 years of continuous specific conductance measurements at USGS station 01104455 (Smith, 2005; Socolow and others, 1999, 2000, 2001, 2002, 2003, and 2004), new maximum monthly mean values were established for 7 months during water year 2005 (fig. 8). 


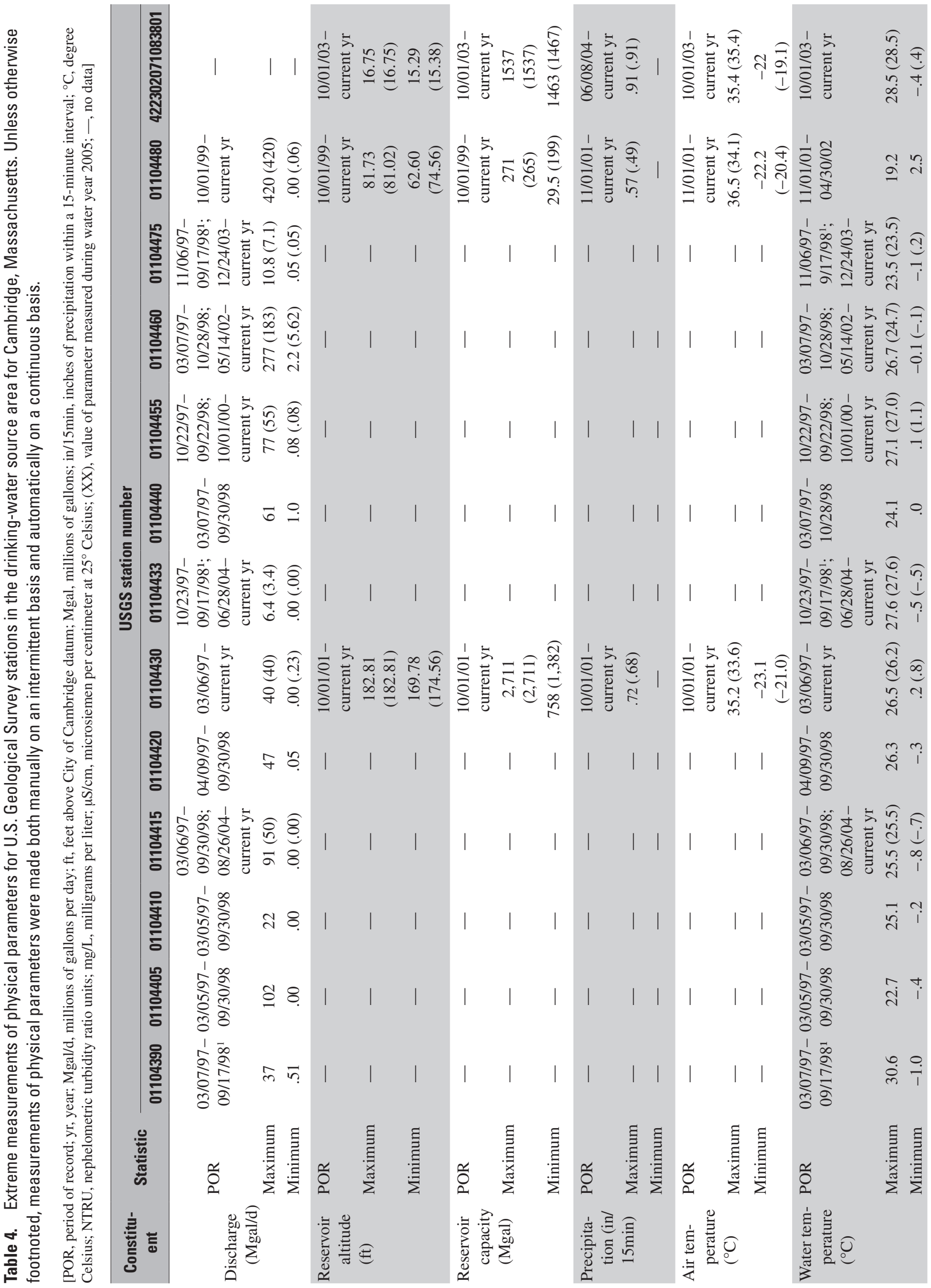




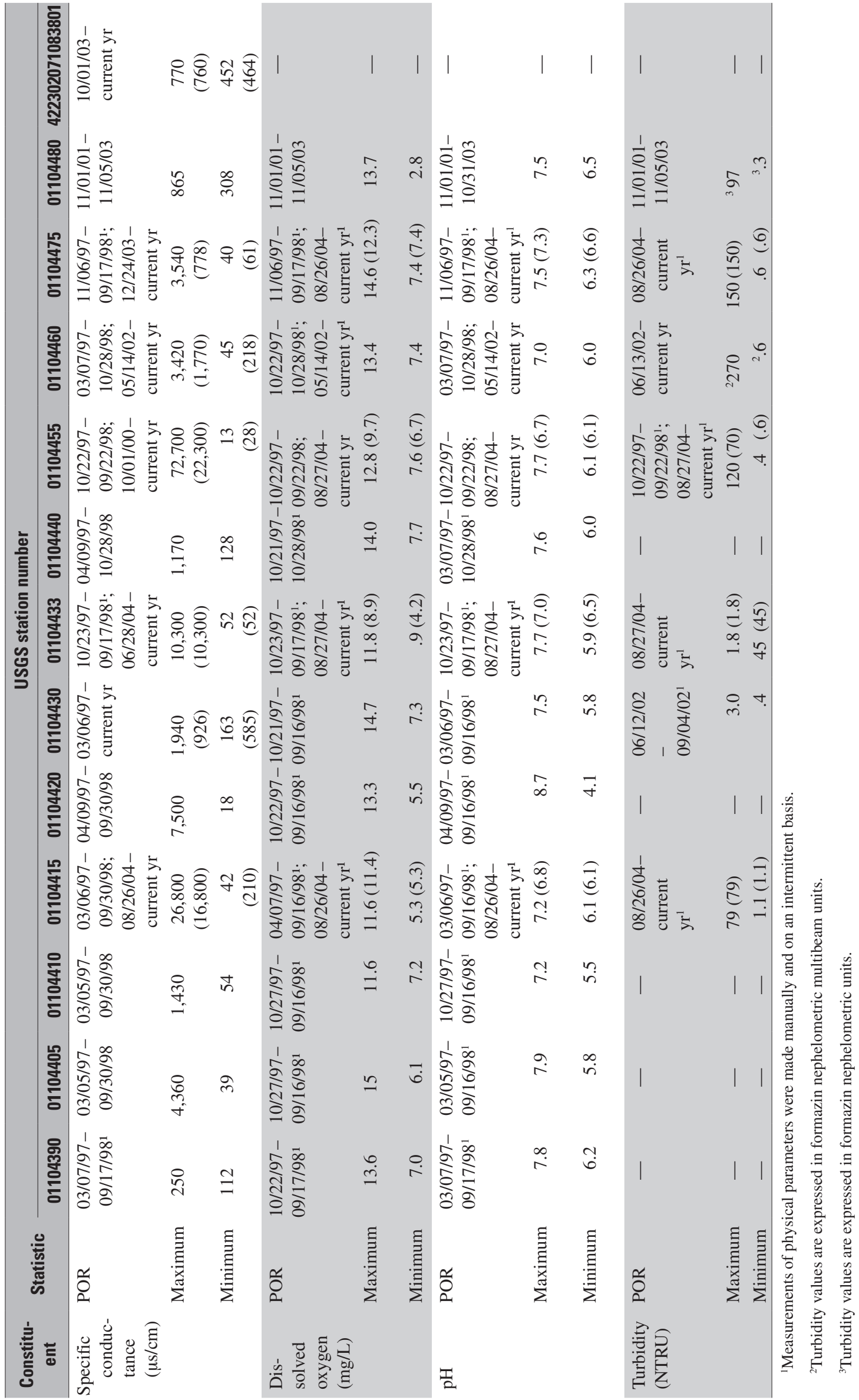




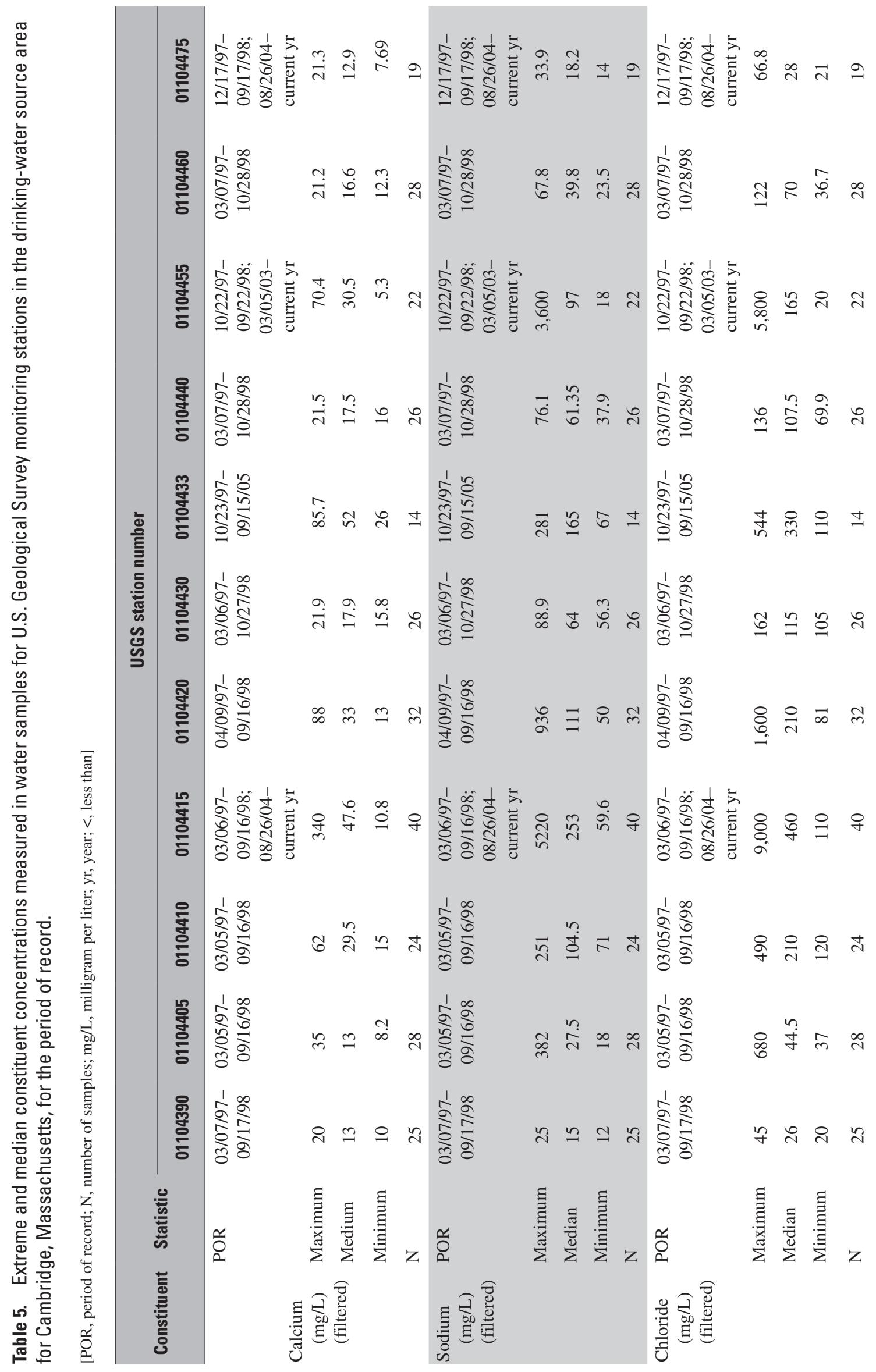




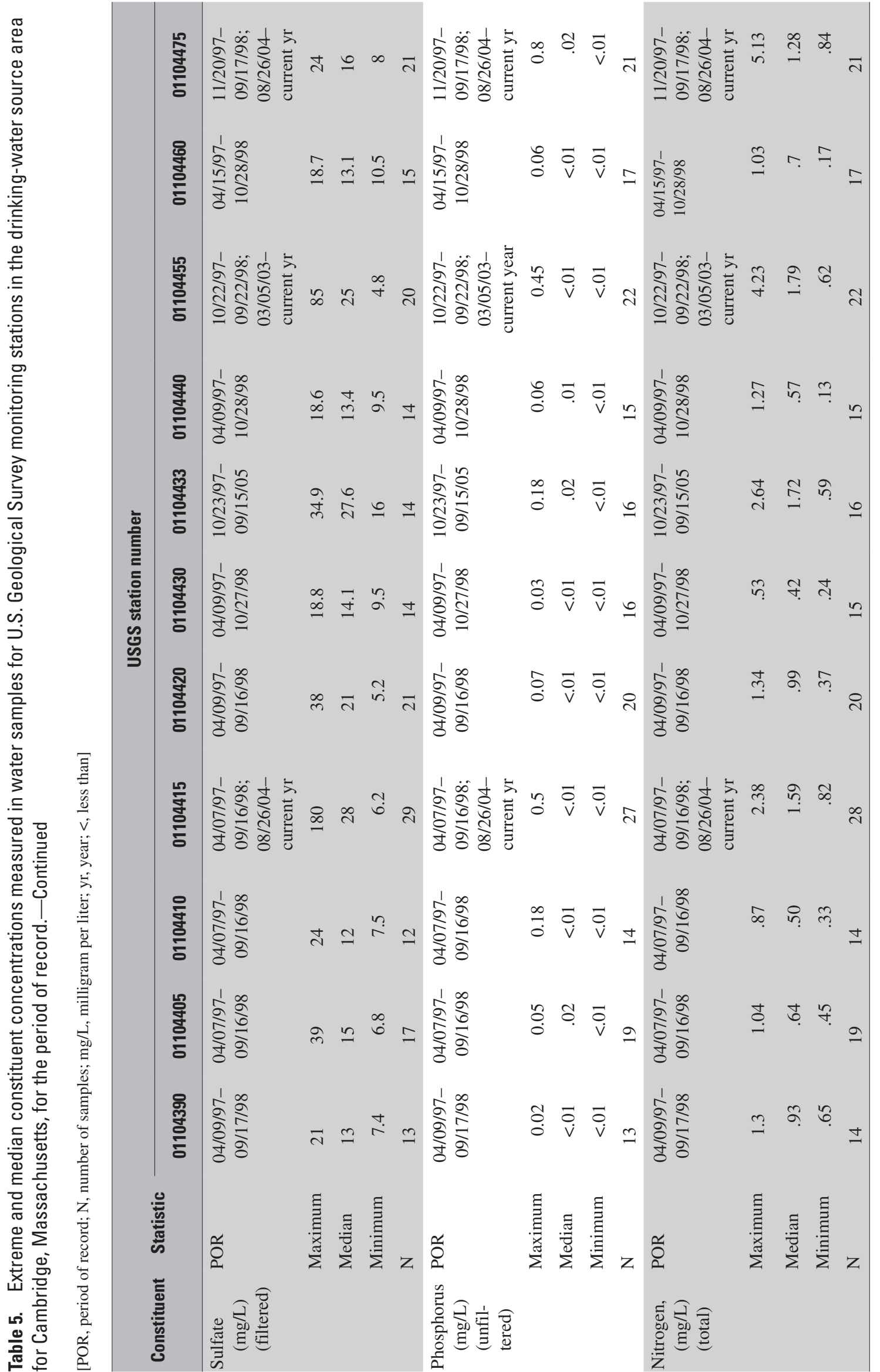




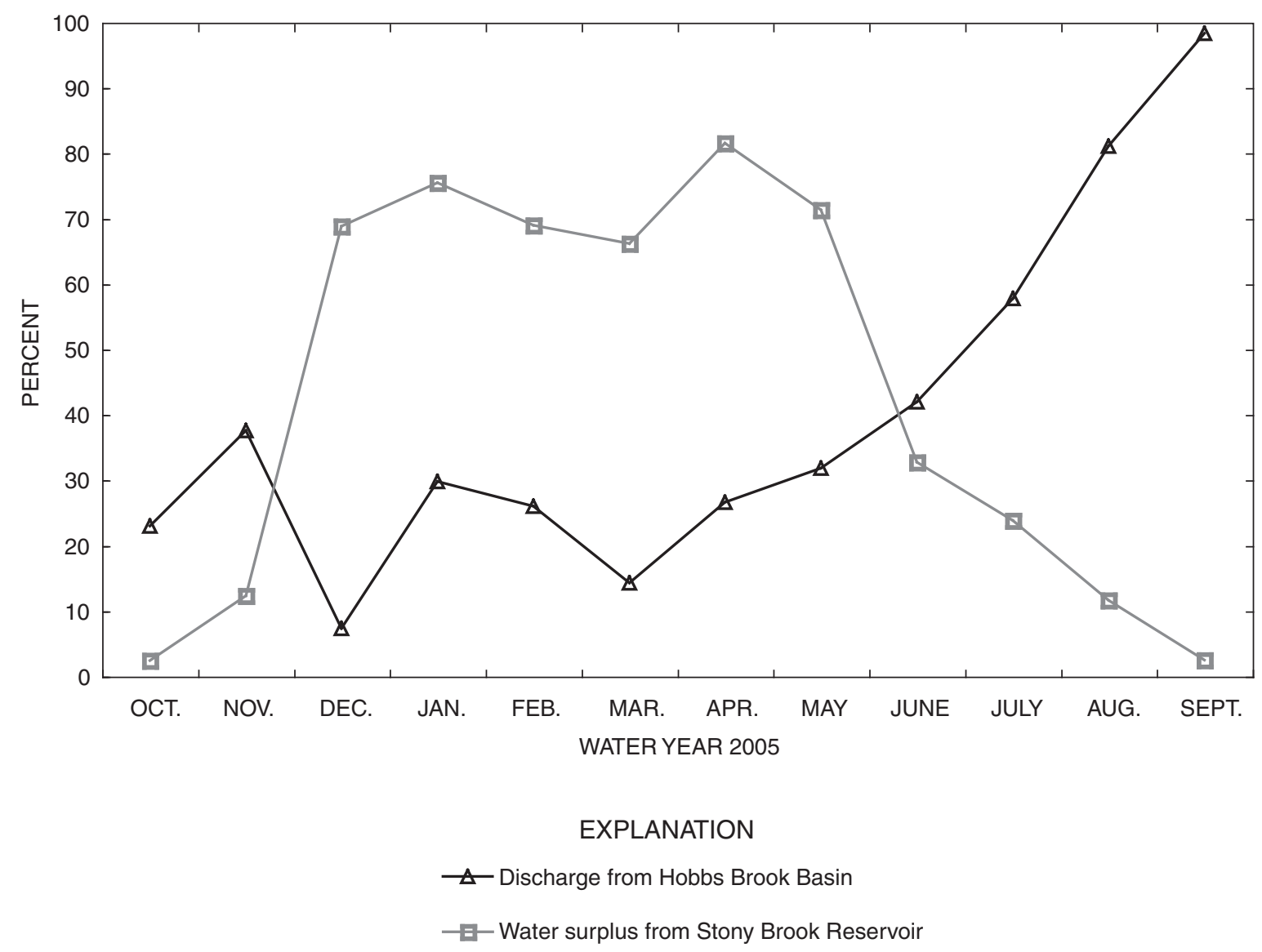

Figure 2. The percentage of water supplied from the Hobbs Brook basin relative to the total water entering the Stony Brook Reservoir compared to the percentage of water discharged from the Stony Brook Reservoir to the Charles River relative to the total inflow for water year 2005, eastern Massachusetts.

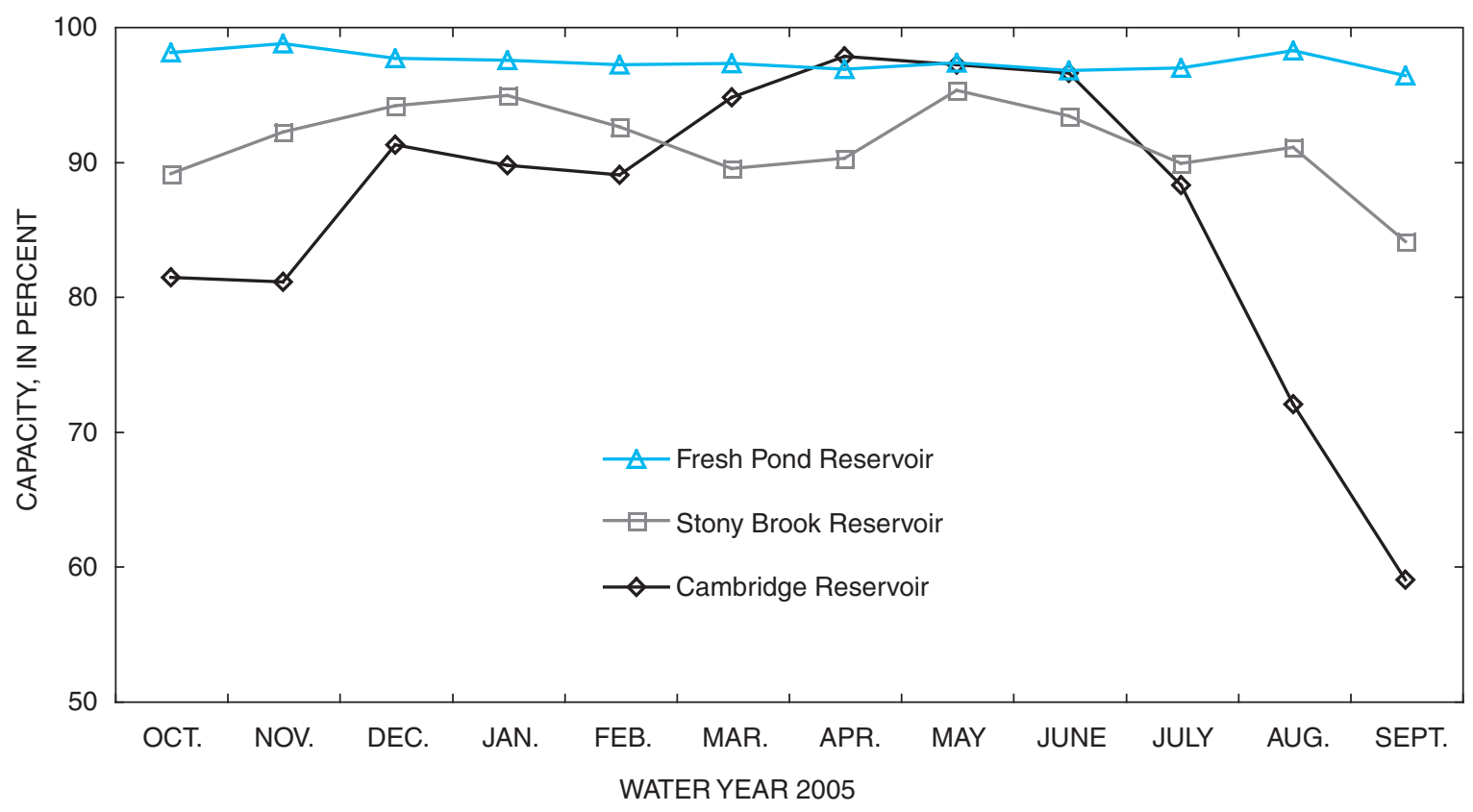

Figure 3. Monthly mean reservoir-storage capacities for water year 2005 shown as percent capacity for the Cambridge Reservoir, Stony Brook Reservoir, and Fresh Pond Reservoir, eastern Massachusetts. 


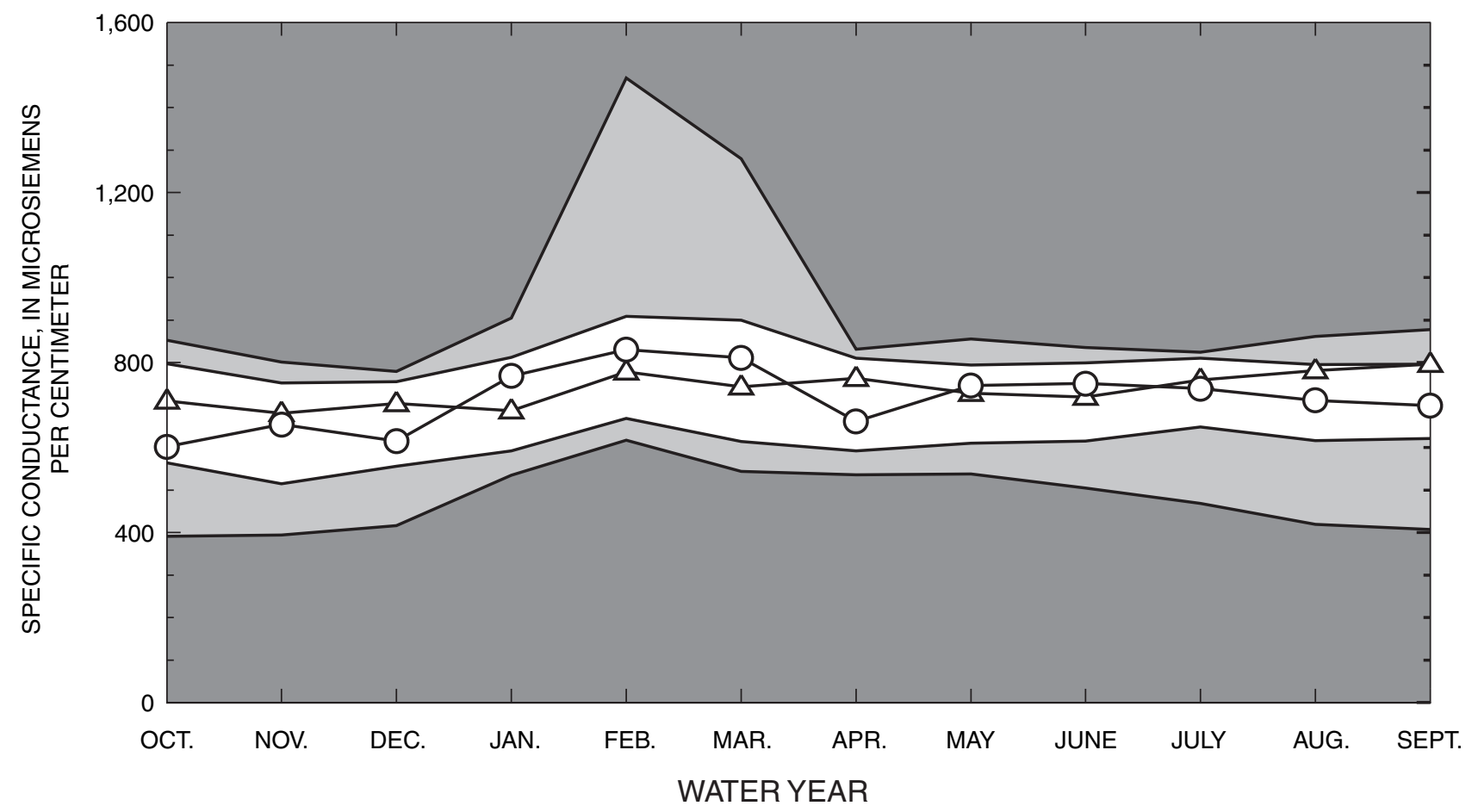

\section{EXPLANATION}

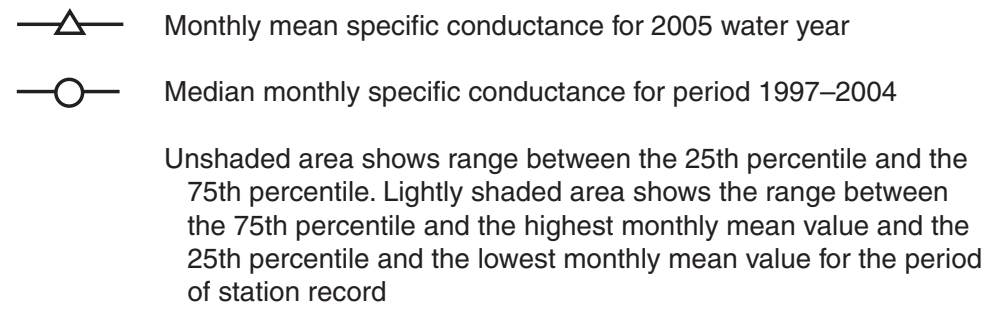

Figure 4. Monthly mean specific conductance for water year 2005 for USGS station 01104430, Hobbs Brook below Cambridge Reservoir near Kendal Green, Massachusetts, compared to the period-of-record maximum and minimum monthly mean specific conductance, and the median monthly specific conductance for water years 1997-2004. 


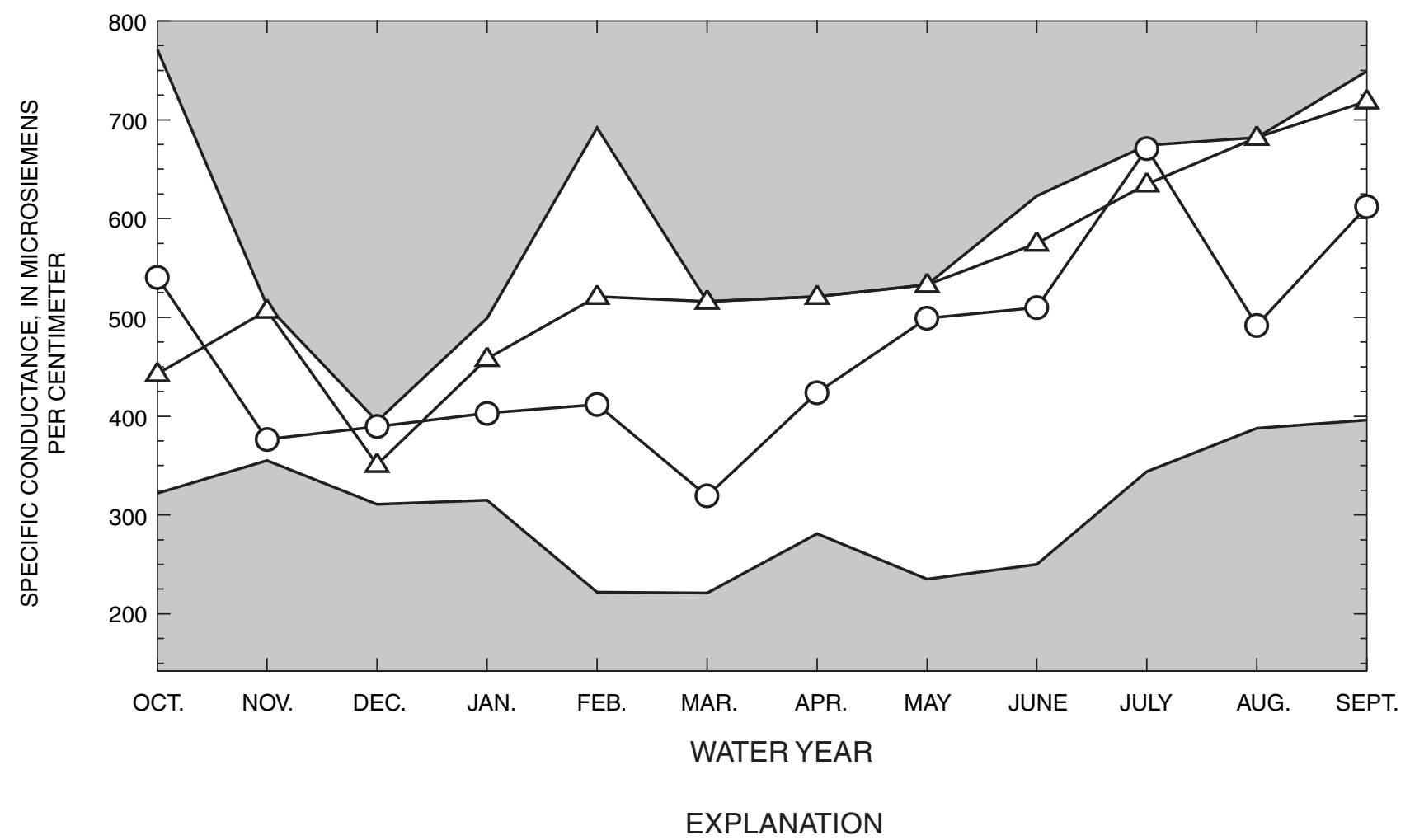

$\triangle \quad$ Monthly mean specific conductance for 2005 water year

- Median monthly specific conductance for period 1997-1998 and 2002-2004

Unshaded area shows range between the highest and lowest monthly mean value for period-of-station record

Figure 5. Monthly mean specific conductance for water year 2005 for USGS station 01104460 , Stony Brook at Route 20 at Waltham, Massachusetts, compared to the period-of-record maximum and minimum monthly mean specific conductance, and the median monthly specific conductance for water years 1997-1998 and 2002-2004. 


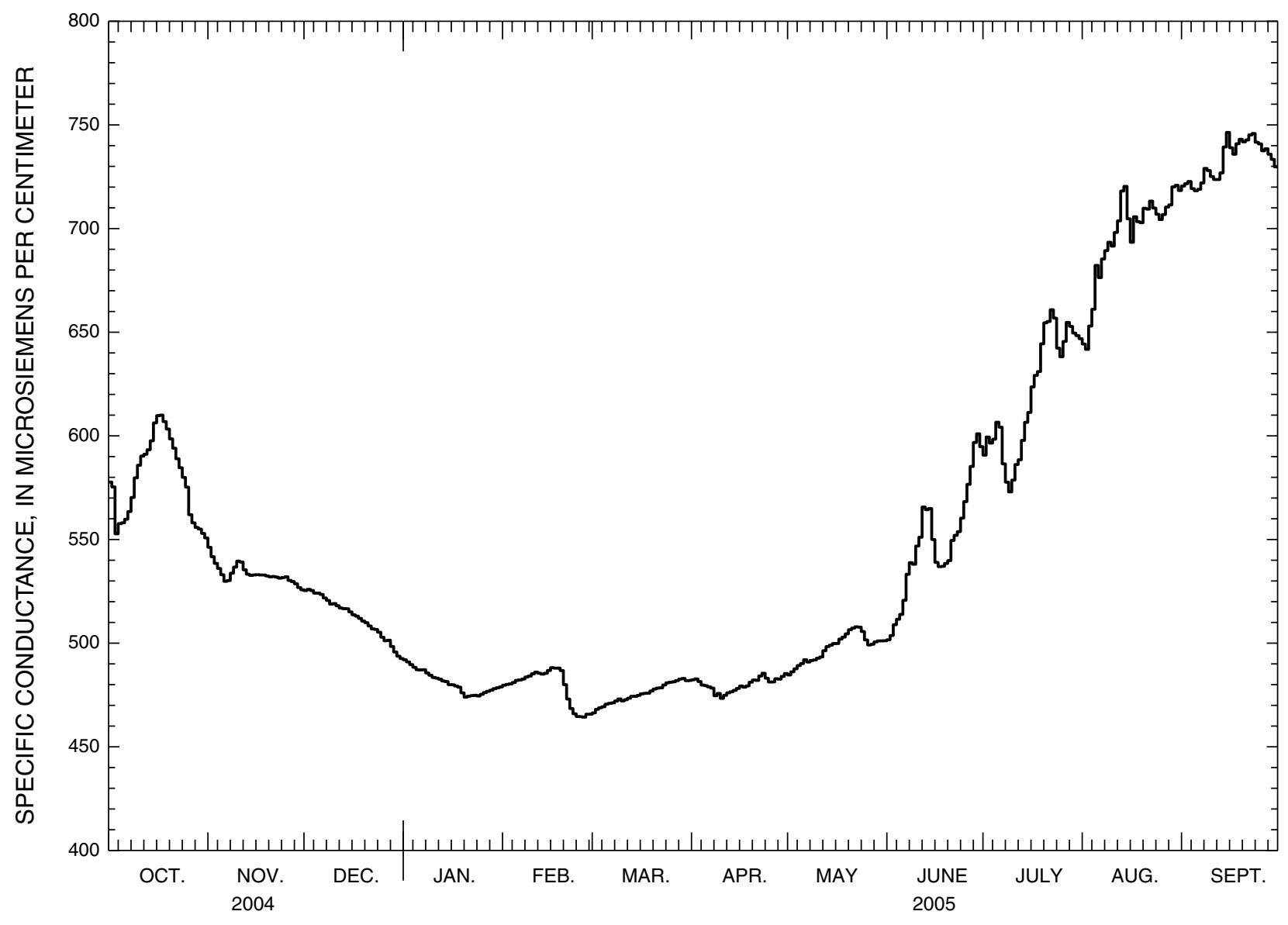

Figure 6. Daily mean specific-conductance values for USGS station 422302071083801, Fresh Pond Reservoir at Cambridge, Massachusetts, for water year 2005. 

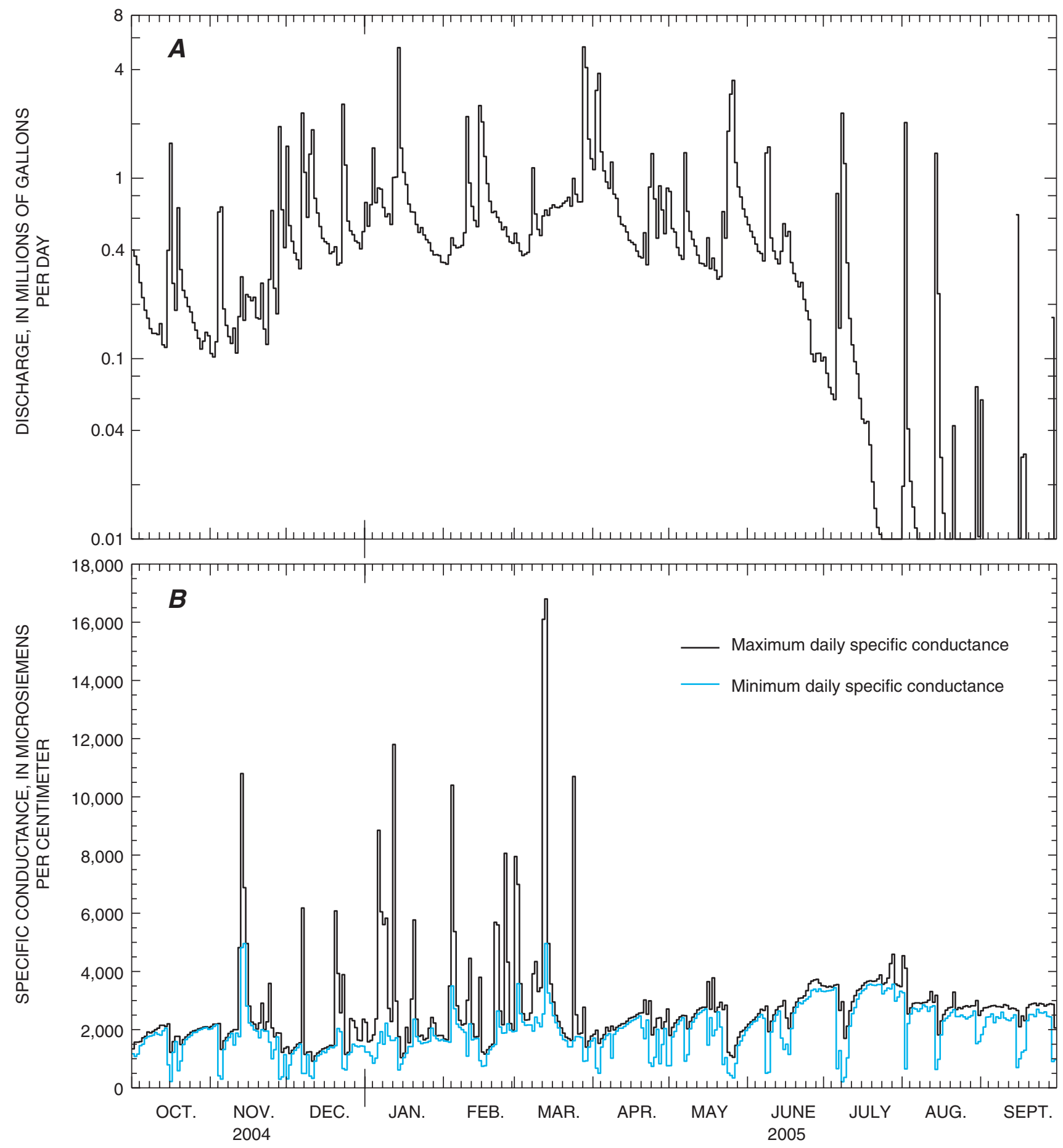

Figure 7. (A) Daily total flows greater than 0.01 million gallons per day, and $(B)$ daily maximum and daily minimum specific conductance values for USGS station 01104415, Cambridge Reservoir, unnamed tributary 2, near Lexington, Massachusetts, for water year 2005. 


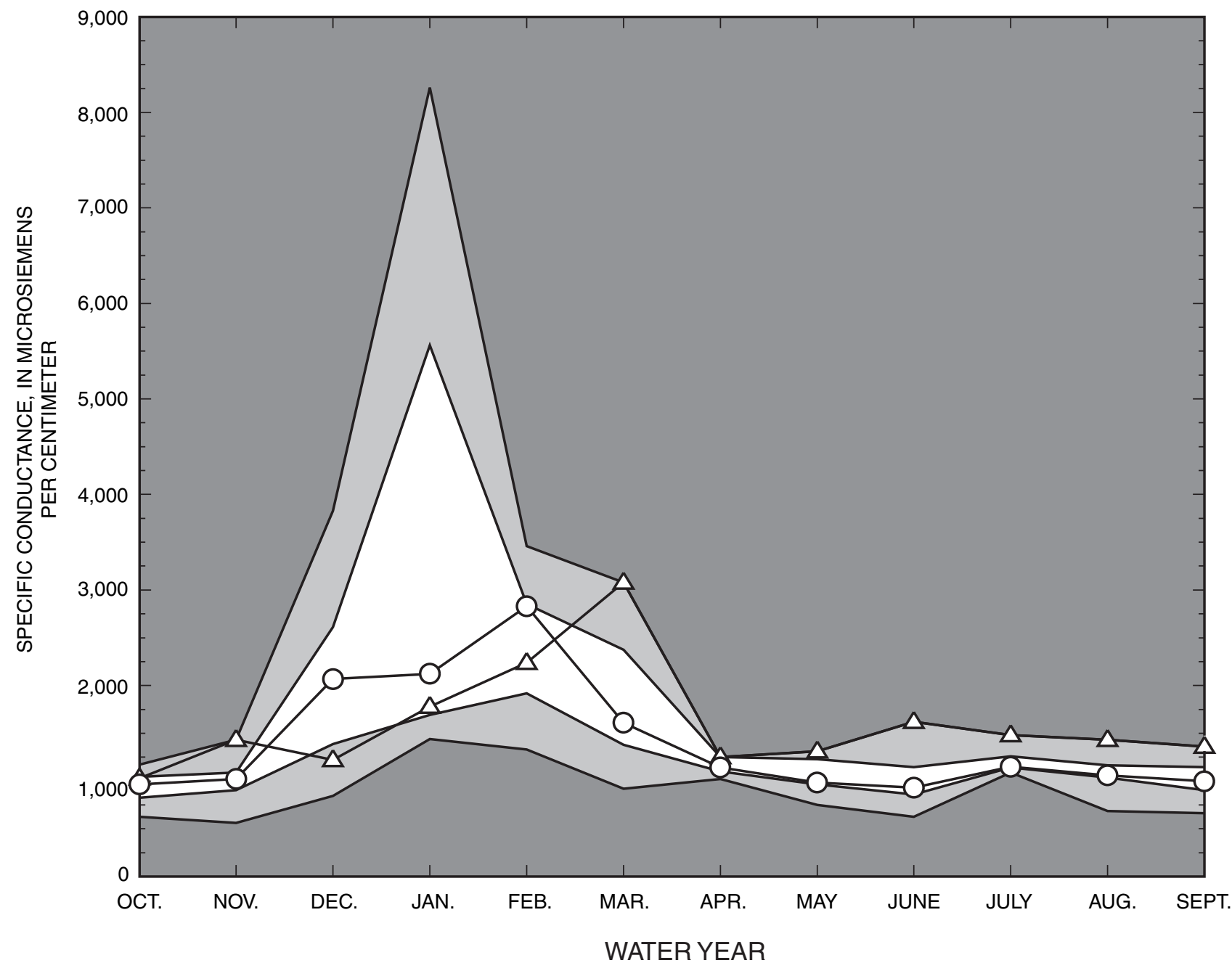

\section{EXPLANATION}

$\triangle \triangle$ Monthly mean specific conductance for 2005 water year

- Median monthly specific conductance for period 1998-2004

Unshaded area shows range between the 25th percentile and the 75th percentile. Lightly shaded area shows the range between the 75th percentile and the highest monthly mean value and the 25th percentile and the lowest monthly mean value for the period of station record

Figure 8. Monthly mean specific conductance for water year 2005 for USGS station 01104455, Stony Brook, unnamed tributary 1, near Waltham, Massachusetts, compared to the period-of-record maximum and minimum monthly mean specific conductance, and the median monthly specific conductance for water years 1998-2004. 


\section{Water Samples}

Concentrations of most dissolved constituents in water samples collected during storms were generally lower than the concentrations observed in water samples collected during base-flow conditions. Nutrient concentrations, including total nitrogen and total phosphorus were similar in samples collected during base-flow conditions for all subbasins; however, phosphorus concentrations were substantially greater in stormwater samples. Most total-recoverable metals were detected in samples of base-flow water as well as in samples of stormwater. Detections of chromium were observed only in samples of stormwater except for one sample collected under base-flow conditions at USGS station 01104415. Lead concentrations were consistently higher in samples of stormwater. With the exception of cadmium and manganese, concentrations of total metals and suspended sediment were generally higher in samples of stormwater than in samples of base-flow water for each of the tributaries.

Iron, manganese, nickel, and zinc were the only metals detected in the field-blank water. Concentrations of iron, manganese, and nickel were at least about an order of magnitude less than the lowest concentration found in the environmental samples collected at the four tributaries. Although the concentration of zinc measured in the field-blank water was low, the results for several environmental samples (particularly those samples collected at USGS station 01104475) also were low and should be viewed with caution. Assuming the concentration of zinc measured in the field-blank water represents the maximum amount of contamination present in any given sample, the potential bias is within the measurement error of the analytical technique (Garbarino and Struzeski, 1998) for environmental samples with concentrations of zinc greater than 20 micrograms per liter $(\mu \mathrm{g} / \mathrm{L})$.

Concentrations of dissolved chloride and total recoverable manganese in samples of base-flow water collected at USGS stations 01104415, 01104433 , and 01104455 during December and August exceeded the USEPA secondary drinking water standards of 250 and $0.05 \mathrm{mg} / \mathrm{L}$ (U.S. Environmental Protection Agency, 2002a), respectively. In samples of stormwater collected at USGS stations 01104415, 01104433, 01104455, and 01104475 in July, August, and September, concentrations of dissolved chloride were below the secondary standard. Conversely, concentrations of total recoverable manganese in all samples of stormwater exceeded the secondary drinking water standard. In half of the water samples collected at USGS stations 01104415, 01104455 and 01104475, concentrations of total recoverable iron exceeded the USEPA secondary drinking water standard of $0.3 \mathrm{mg} / \mathrm{L}$ (U.S. Environmental Protection Agency, 2002a). Concentrations of total recoverable iron in all four samples collected at USGS station 01104433 exceeded the secondary standard. In several cases, the concentrations of total recoverable iron in water samples collected during storms exceeded the standard by more than an order of magnitude.
National secondary drinking water regulations are non-mandatory water-quality standards established by the USEPA. They are guidelines designed to assist public water suppliers in managing their drinking water for aesthetic considerations, such as taste, odor, color, foaming, corrosivity, staining, scaling, and sedimentation. These constituents do not present a risk to human health at the secondary maximum contaminant level (U.S. Environmental Protection Agency, 2002a). Although these standards are typically applied to finished water, the presence of these constituents in concentrations that exceed the respective standards can damage water equipment or reduce the effectiveness of treatment for other contaminants. Some constituents, such as chloride, sodium, and nitrogen, cannot be effectively removed or reduced during the water treatment process (table 15).

Concentrations of Escherichia coli bacteria in water samples of base flow ranged from 4 to 1,400 colony-forming units per $100 \mathrm{~mL}(\mathrm{col} / 100 \mathrm{~mL})$. Bacterial concentrations were lower in water samples of base flow collected in December compared to water samples of base flow collected in July. Concentrations of Escherichia coli bacteria in composite samples of stormwater ranged between 1,700 to 43,000 col/100mL) with the highest concentrations measured at USGS station 01104475. Bacteria concentrations in all equipment-blank samples were less than the detection limit. The difference between concentrations of bacteria in replicate split samples ranged from 5 to 91 percent.

Fluoranthene, $9 \mathrm{H}$-fluorene, acenaphthene, anthracene, phenanthrene, and pyrene were the only PAHs detected in samples of base-flow water. Fluoranthene and pyrene were the most commonly detected compounds in base-flow samples. Concentrations of PAH compounds observed in composite samples of stormwater were higher, often as much as an order of magnitude or more, than concentrations measured in samples of base-flow water. With the exception of nitrobenzene, which was the only compound not detected at a concentration of $2 \mu \mathrm{g} / \mathrm{L}$ in any sample, all PAH compounds were detected at all subbasins in one or both composite samples of stormwater. Fluoranthene, phenanthrene, and pyrene were the only PAH compounds found in the water sample collected from the Fresh Pond Reservoir intake structure. None of these compounds were detected at the respective detection limits (table 15) in the finished drinking water.

Naphthalene was the only PAH compound detected in the field blank. The field blank concentration was about 2 times greater than the environmental sample concentrations, and as a result, the environmental results should be viewed with caution. One possible explanation for the detection of naphthalene in the field blank is that sodium naphthalene is used in the manufacturing of some Teflon products (Benderly, 1962). Because the source water was stored in a bottle lined with a Teflon bag for nearly 14 months, it is possible that the naphthalene leached from the bag and therefore the concentrations found in the environmental samples may be in fact valid.

Sixteen pesticides and caffeine were detected in water samples collected in four tributaries and in raw water collected 
from the Cambridge water-treatment facility intake from the Fresh Pond Reservoir in water year 2005. Most of these pesticides are found in over-the-counter products used for turf management and the treatment of ornamental shrubs (table 6). Caffeine, 2,4-D, imidacloprid, and siduron were the only compounds detected in all four subbasins. Carbaryl and metsulfuron were detected in three of the four subbasins. Caffeine, imidacloprid, and siduron were also detected in water from the Fresh Pond Reservoir. The compounds 3-ketocarbofuran, carbaryl, MCPA, propoxur, siduron, and triclopyr were only detected in water samples collected from storm-water runoff. Caffeine, imidacloprid, and siduron were the most frequently detected compounds (table 7).

\section{Meteorological Data}

The distribution of monthly precipitation totals for the USGS station at the Cambridge Reservoir (fig. 9) was more uniform during the 2005 water year than during any other water year for the period of record (Smith, 2005; Socolow and others, 2002, 2003, and 2004). The COVs for monthly precipitation totals for the three stations that measured precipitation ranged from about 32 to 34 percent. Monthly precipitation totals for USGS stations at the Stony Brook Reservoir and the
Fresh Pond Reservoir differed by -10 to 47 percent compared to the USGS station at the Cambridge Reservoir. Average differences compared to the station at the Cambridge Reservoir were about 19 and 26 percent, respectively. In general, variability between monthly precipitation totals for all stations is attributed to differences in storm tracks, storm intensity patterns, and storm wind intensity. Additionally, variability between monthly precipitation totals during the winter months is compounded by a change in precipitation type, that is, snow is less dense and therefore affected more by wind. The precipitation gages at the Stony Brook Reservoir and Fresh Pond Reservoir are more susceptible to wind bias and therefore the totals are generally lower (fig. 9). The annual precipitation total recorded at the Cambridge Reservoir during water year 2005 was within 2 inches of the totals recorded during the previous two water years.

Mean monthly air temperature values were similar from site to site, differing by no more than about $1.5^{\circ} \mathrm{C}$. Mean monthly air temperature values for the USGS station at the Cambridge Reservoir had the greatest amount of variability in the months of January and February (fig. 10). During the 2005 water year, mean monthly air temperature values were within $1{ }^{\circ} \mathrm{C}$ of the median values for water years 2002 through 2004, except for the months of January, March, May, and June. 


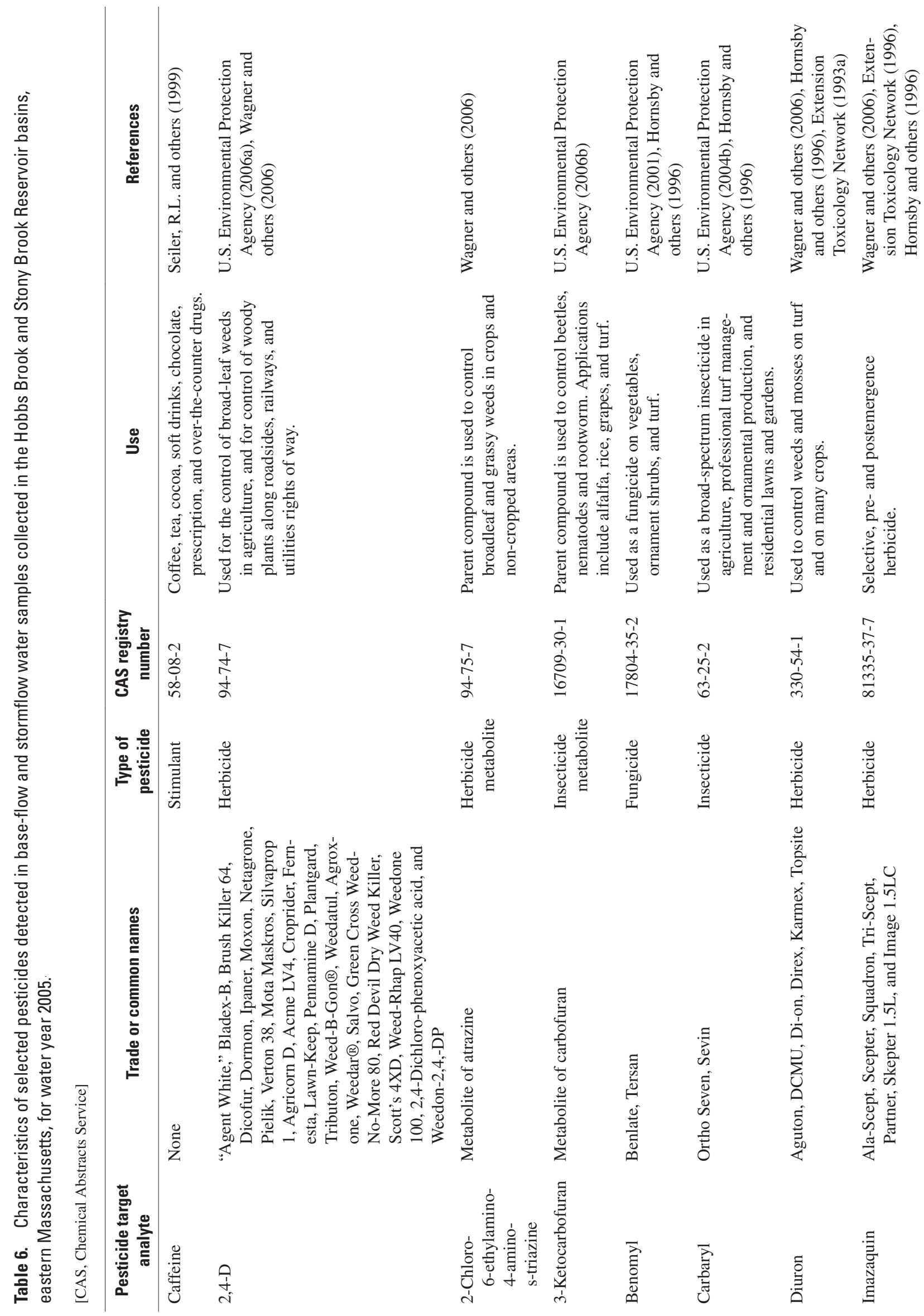



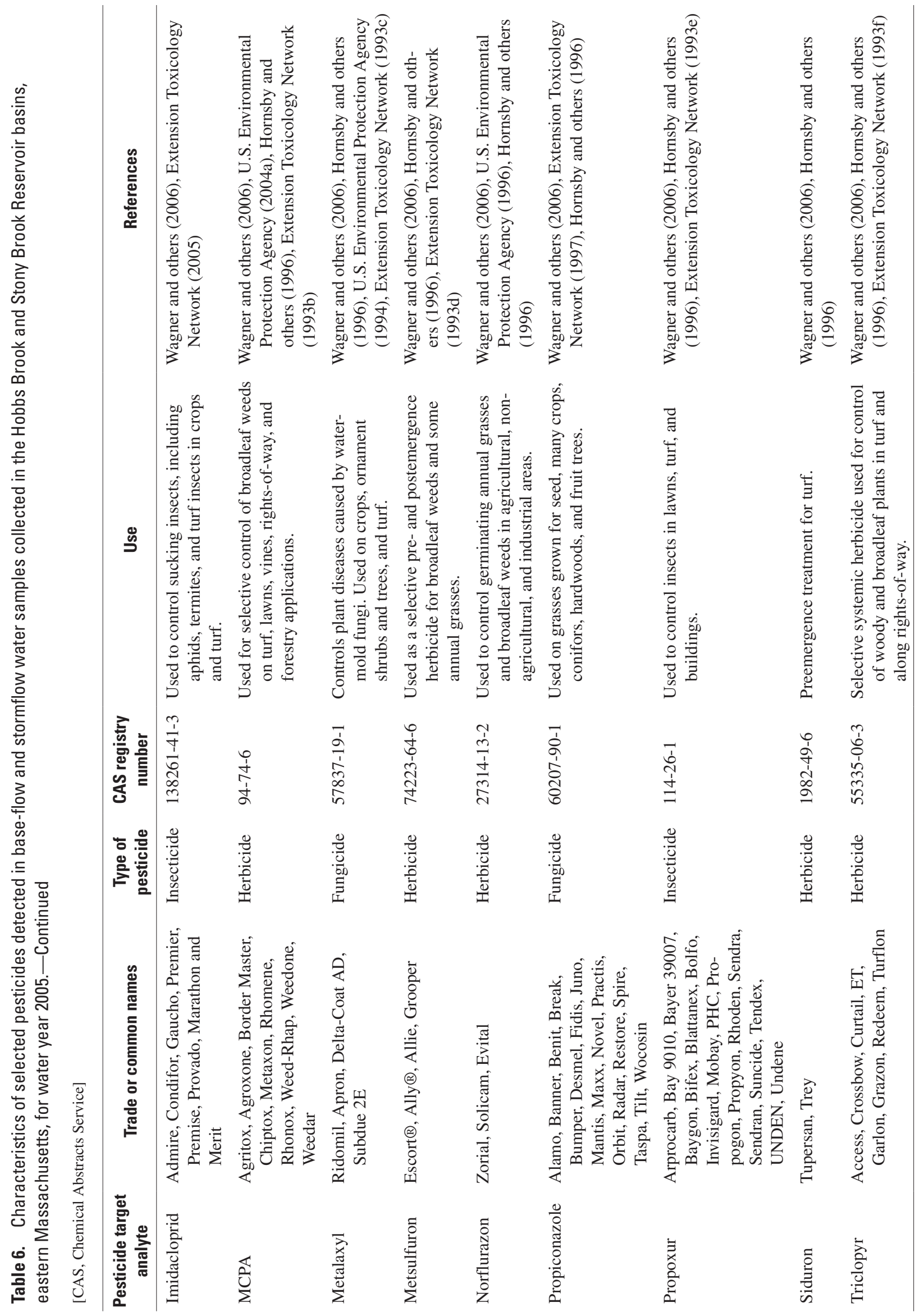
Table 7. Frequency of detection and maximum concentration of selected pesticides detected in base-flow and stormflow water samples collected in the Hobbs Brook and Stony Brook Reservoir basins, eastern Massachusetts, for water year 2005.

[E, the recovery or variation in recovery of the analyte was outside the acceptable range, or concentration reported is less than laboratory reporting level and is qualified as estimated]

\begin{tabular}{lccc}
\hline Pesticide target analyte & $\begin{array}{c}\text { Frequency of detection for } \\
\text { water year 2005 } \\
\text { (percent) }\end{array}$ & $\begin{array}{c}\text { Number of } \\
\text { samples }\end{array}$ & $\begin{array}{c}\text { Maximum concentration } \\
\text { (micrograms per liter) }\end{array}$ \\
\hline Caffeine & 81 & 16 & E1.69 \\
2,4-D & 31 & 16 & E7.92 \\
2-Chloro-6-ethylamino-4-amino-s-triazine & 6 & 16 & E.01 \\
3-Ketocarbofuran & 8 & 12 & E.08 \\
Benomyl & 19 & 16 & E.176 \\
Carbaryl & 25 & 16 & .03 \\
Diuron & 25 & 16 & E.14 \\
Imazaquin & 19 & 16 & E.06 \\
Imidacloprid & 63 & 16 & E1.00 \\
MCPA & 8 & 12 & E.16 \\
Metalaxyl & 19 & 16 & E.04 \\
Metsulfuron & 25 & 16 & E3.90 \\
Norflurazon & 19 & 16 & E.15 \\
Propiconazole & 19 & 16 & E.54 \\
Propoxur & 6 & 16 & E2.46 \\
Siduron & 44 & 16 & E1.44 \\
Triclopyr & 13 & 16 & \\
\hline
\end{tabular}




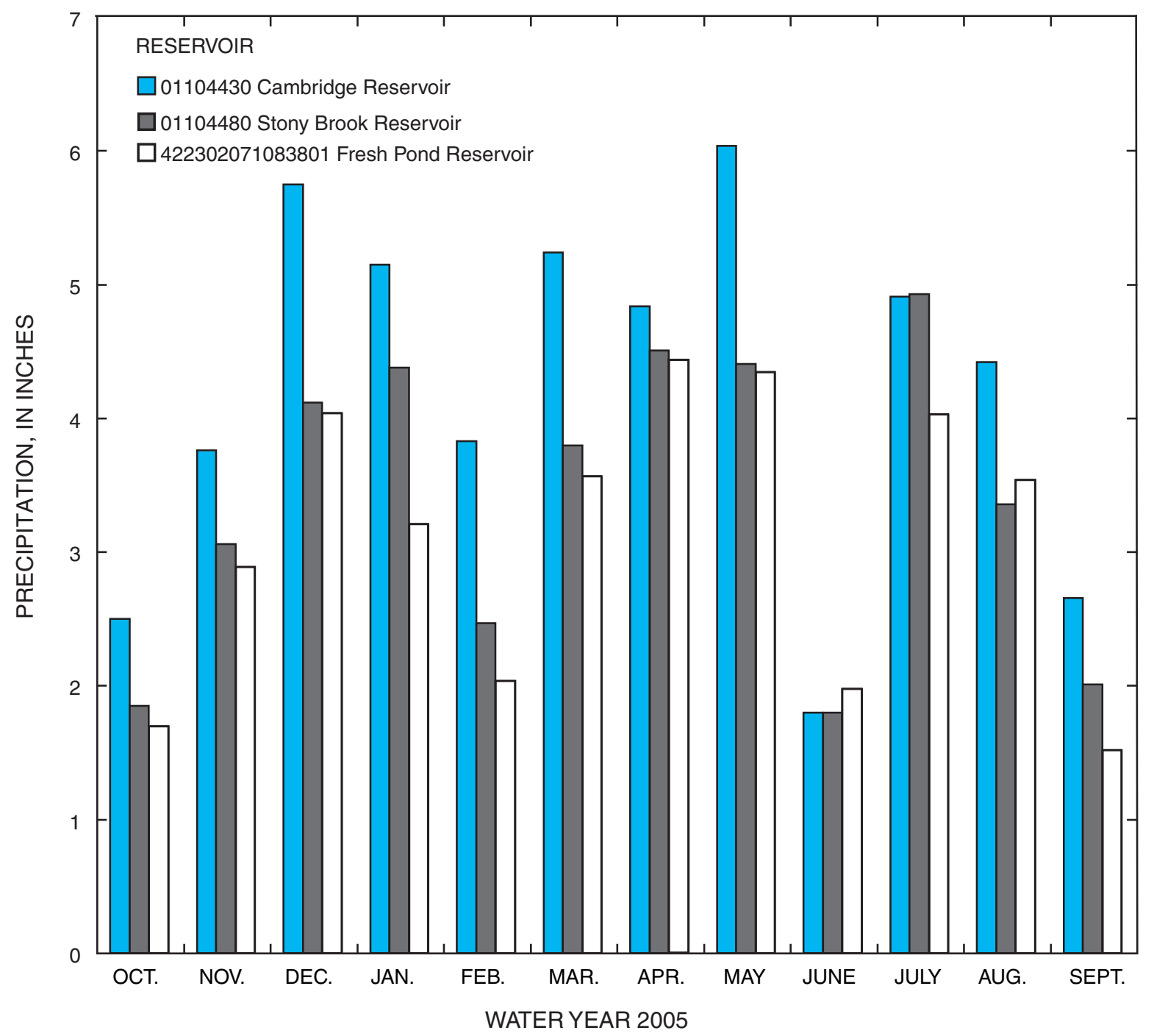

Figure 9. Monthly precipitation totals for the Cambridge Reservoir, Stony Brook Reservoir, and Fresh Pond Reservoir in the Cambridge, Massachusetts, drinking-water source area for water year 2005. 


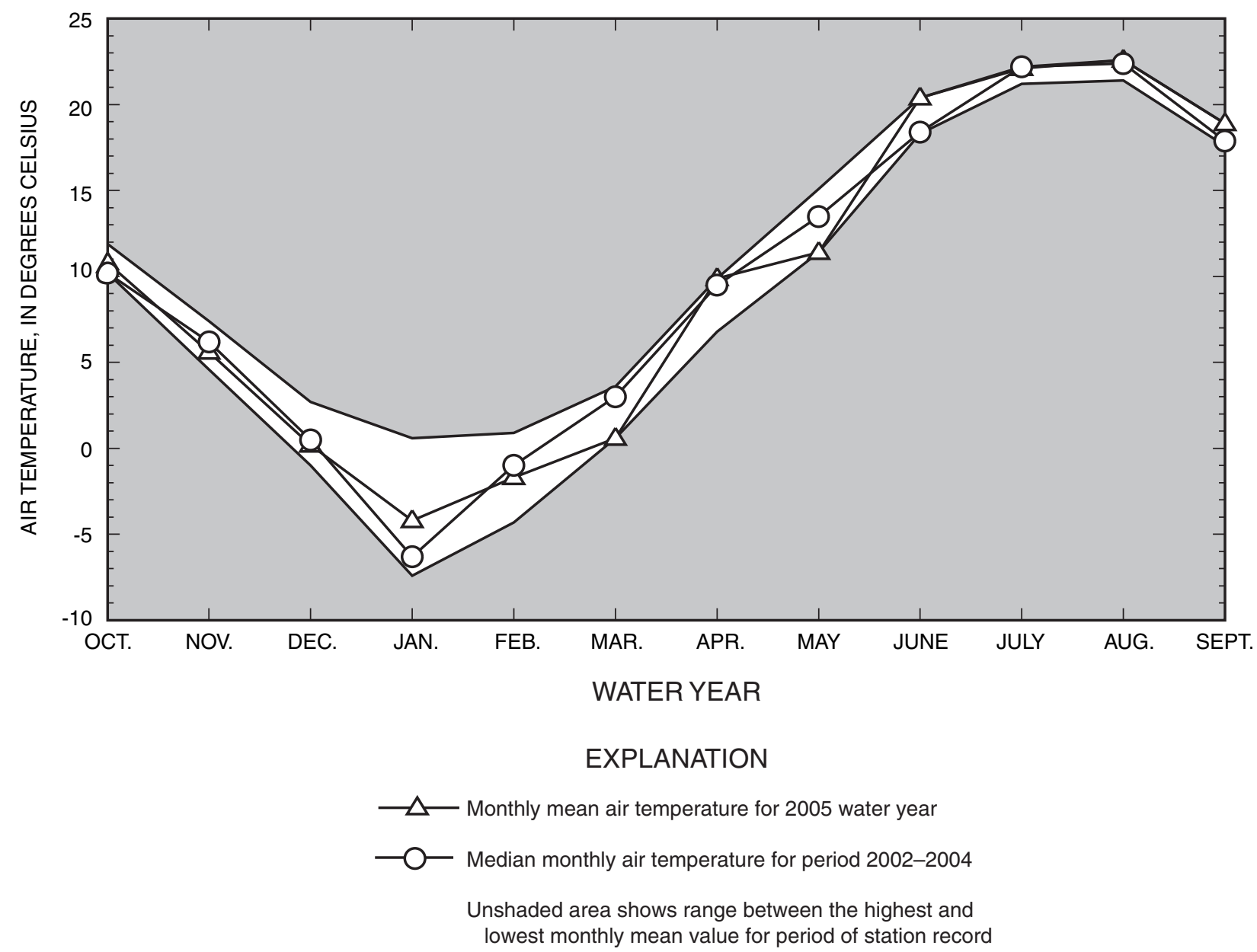

Figure 10. Monthly mean air temperatures for water year 2005 for the Cambridge Reservoir, compared to the period-ofrecord maximum and minimum monthly mean air temperatures and the median monthly air temperatures for water years 2002-2004, eastern Massachusetts. 


\section{References Cited}

American Public Health Association, 1998, Standard methods for the examination of water and wastewater (20th ed.); Washington, D.C., American Public Health Association, American Water Works Association, and Water Environment Federation, p. 3-37-3-43.

Anderson, C.W., 2004 (2d ed.), Turbidity, in Wilde, F.D., and Radtke, D.B., eds., Field measurements: U.S. Geological Survey Techniques of Water-Resources Investigations, book 9, chap. A6.7, 64 p., accessed April 7, 2005, at http://water.usgs.gov/owq/FieldManual/Chapter6/6.7 contents.htm

ASTM International, 2003, D1889-00 Standard test method for turbidity of water, in ASTM International, Annual Book of ASTM Standards, Water and Environmental Technology, v. 11.01: West Conshohocken, Pennsylvania, 6 p.

Benderly, A.A., 1962, Treatment of Teflon to promote bondability: Journal of Applied Polymer Science, v. 6, no. 20, p. 221-225.

Buchanan, T.J., and Somers, W.P., 1968, Stage measurements at gaging stations: Techniques of Water-Resources Investigations of the U.S. Geological Survey, book 3, chap. A7, $28 \mathrm{p}$.

Buchanan, T.J., and Somers, W.P., 1969, Discharge measurements at gaging stations: Techniques of Water-Resources Investigations of the U.S. Geological Survey, book 3, chap. A8, 65 p.

Carter, R.W., and Davidian, Jacob, 1968, General procedure for gaging streams: Techniques of Water-Resources Investigations of the U.S. Geological Survey, book 3, chap. A6, $13 \mathrm{p}$.

Extension Toxicology Network, 2005, Pesticide information profile for imidacloprid, accessed on April 05, 2006, at http://pmep.cce.cornell.edu/profiles/extoxnet/haloxyfopmethylparathion/imidacloprid-ext.html

Extension Toxicology Network, 1997, Pesticide information profile for propiconazole, accessed on April 05, 2006, at http://pmep.cce.cornell.edu/profiles/extoxnet/metirampropoxur/propiconazole-ext.html

Extension Toxicology Network, 1996, Pesticide information profile for imazaquin, accessed on April 05, 2006, at http://pmep.cce.cornell.edu/profiles/extoxnet/haloxyfopmethylparathion/imazaquin-ext.htm

Extension Toxicology Network, 1993a, Pesticide information profile for diuron, accessed on April 05, 2006, at http://pmep.cce.cornell.edu/profiles/extoxnet/dienochlorglyphosate/diuron-ext.htm
Extension Toxicology Network, 1993b, Pesticide information profile for MCPA, accessed on April 05, 2006, at http://pmep.cce.cornell.edu/profiles/extoxnet/haloxyfopmethylparathion/mcpa-ext.htm

Extension Toxicology Network, 1993c, Pesticide information profile for metalaxyl, accessed on April 05, 2006, at http://pmep.cce.cornell.edu/profiles/extoxnet/haloxyfopmethylparathion/metalaxyl-ext.html

Extension Toxicology Network, 1993d, Pesticide information profile for metsulfuron-methyl, accessed on April 05, 2006, at http://pmep.cce.cornell.edu/profiles/extoxnet/metirampropoxur/metsulfuron-methyl-ext.html

Extension Toxicology Network, 1993e, Pesticide information profile for propoxur, accessed on April 05, 2006, at http://pmep.cce.cornell.edu/profiles/extoxnet/metirampropoxur/propoxur-ext.html

Extension Toxicology Network, 1993f, Pesticide information profile for triclopyr, accessed on April 05, 2006, at http://pmep.cce.cornell.edu/profiles/extoxnet/pyrethrinsziram/triclopyr-ext.html

Fishman, M.J., ed., 1993, Methods of analysis by the U.S. Geological Survey National Water Quality LaboratoryDetermination of inorganic and organic constituents in water and fluvial sediments: U.S. Geological Survey OpenFile Report 93-125, 217 p.

Fishman, M.J., and Friedman, L.C., 1989, Methods for determination of inorganic substances in water and fluvial sediments: U.S. Geological Survey Techniques of WaterResources Investigations, book 5, chap. A1, 545 p.

Fugro East, Inc., 1996, Limnological investigations of the Cambridge Reservoir system-Impacts of watershed inputs and alum sludge discharge: Cambridge, Mass., Cambridge Water Department, 77 p., plus appendixes.

Furlong, E.T., Anderson, B.D., Werner, S.L., Soliven, P.P., Coffey, L.J., and Burkhardt, M.R., 2001, Methods of analysis by the U.S. Geological Survey National Water Quality Laboratory-Determination of pesticides in water by graphitized carbon-based solid-phase extraction and high-performance liquid chromatography/mass spectrometry: U.S. Geological Survey Water-Resources Investigations Report 01-4134, 73 p.

Furlong, E.T., Vaught, D.G., Merten, L.M., Foreman, W.T., and Gates, P.M., 1996, Methods of analysis by the U.S. Geological Survey National Water Quality LaboratoryDetermination of semivolatile organic compounds in bottom sediment by solvent extraction, gel permeation chromatographic fractionation, and capillary-column gas chromatography/mass spectrometry: U.S. Geological Survey OpenFile Report 95-719, 67 p. 
Garbarino, J.R., and Struzeski, T.M., 1998, Methods of analysis by the U.S. Geological Survey National Water Quality Laboratory-Determination of elements in whole-water digests using inductively coupled plasma-optical emission spectrometry and inductively coupled plasma-mass spectrometry: U.S. Geological Survey Open-File Report 98-165, $101 \mathrm{p}$.

Guy, H.P., 1970, Fluvial sediment concepts: U.S. Geological Survey Techniques of Water-Resources Investigations, book 3, chap. C1, 55 p.

Hoffman, G.L., Fishman, M.J., and Garbarino, J.R., 1996, Methods of analysis by the U.S. Geological Survey National Water Quality Laboratory-In-bottle acid digestion of whole-water samples: U.S. Geological Survey Open-File Report 96-225, 28 p.

Hornsby, A.G., Wauchope, R.D., and Herner, A.E., 1996, Pesticide properties in the environment: New York, Springer-Verlag, 227 p.

Kennedy, E.J., 1983, Computation of continuous records of streamflow: Techniques of Water-Resources Investigations of the U.S. Geological Survey, book 3, chap. A13, 52 p.

Kennedy, E.J., 1984, Discharge ratings at gaging stations: Techniques of Water-Resources Investigations of the U.S. Geological Survey, book 3, chap. A10, 59 p.

McLain, Betty, 1993, Methods of analysis by the U.S. Geological Survey National Water Quality Laboratory-Determination of chromium in water by graphite furnace atomic absorption spectrophotometry: U.S. Geological Survey Open-File Report 93-449, 16 p.

Myers, D.N., and Sylvester, M.A., 1997, National field manual for the collection of water-quality data-Biological indicators: U.S. Geological Survey Techniques of WaterResources Investigations, book 9, chap. A7, variously paged.

Oberg, K.A., Morlock, S.E., and Caldwell, W.S., 2005, Quality-assurance plan for discharge measurements using acoustic Doppler current profilers: U.S. Geological Survey Scientific Investigations Report 2005-5183, 35 p.

Patterson, Glenn, 1997, The U.S. Geological Survey drinking water initiative: U.S. Geological Survey Fact Sheet 047-97, 2 p.

Patton, C.J., and Kryskalla, J.R., 2003, Methods of analysis by the U.S. Geological Survey National Water Quality Laboratory-Evaluation of alkaline persulfate digestion as an alternative to kjeldahl digestion for determination of total and dissolved nitrogen and phosphorus in water: U.S. Geological Survey Water-Resources Investigations Report 03-4174, 33 p.
Rantz, S.E. and others, 1982, Measurement and computation of streamflow-Volume 1. Measurement of stage and discharge: U.S. Geological Survey Water Supply Paper 2175 , 284 p.

Seiler, R.L., Zaugg, S.D., Thomas, J.M., and Howcroft, D.L., 1999, Caffeine and pharmaceuticals as indicators of waste water contamination in wells: Ground Water, v. 37, no. 3, p. $405-410$.

Sholar, C.J., and Shreve, E.A., 1998, Quality-assurance plan for the analysis of fluvial sediment by the northeastern region, Kentucky District Sediment Laboratory: U.S. Geological Survey Open-File Report 98-348, 20 p.

Smith, K.P., 2005, Hydrologic, water-quality, bed-sediment, soil-chemistry, and statistical summaries of data for the Cambridge, Massachusetts, drinking-water source area, water year 2004: U.S. Geological Survey Open-File Report 2005-1383, 110 p.

Socolow, R.S., Comeau, L.Y., Zanca, J.L., and Ramsbey, L.R., 1999, Water-resources data for Massachusetts and Rhode Island, water year 1998: U.S. Geological Survey WaterData Report MA-RI-98-1, 438 p.

Socolow, R.S., Girouard, G.G., Whitley, J.F., and Ramsbey, L.R., 2003, Water-resources data for Massachusetts and Rhode Island, water year 2002: U.S. Geological Survey Water-Data Report MA-RI-02-1, 339 p.

Socolow, R.S., Leighton, J.F., Whitley, J.F., and Ventetuolo, D.J., 2002, Water-resources data for Massachusetts and Rhode Island, water year 2001: U.S. Geological Survey Water-Data Report MA-RI-01-1, 307 p.

Socolow, R.S., Whitley, J.S., Murino, Jr. D., and Ramsbey, L.R., 2001, Water-resources data for Massachusetts and Rhode Island, water year 2000: U.S. Geological Survey Water-Data Report MA-RI-00-1, 459 p.

Socolow, R.S., Zanca, J.L., Driskell, T.R., and Ramsbey, L.R., 2004, Water-resources data for Massachusetts and Rhode Island, water year 2003: U.S. Geological Survey WaterData Report MA-RI-03-1, 368 p.

Socolow, R.S., Zanca, J.L., Murino, Jr. D., and Ramsbey, L.R., 2000, Water-resources data for Massachusetts and Rhode Island, water year 1999: U.S. Geological Survey WaterData Report MA-RI-99-1, 401 p.

U.S. Environmental Protection Agency, 2006a, Consumer factsheet on 2,4-D, accessed on April 05, 2006, at http://www.epa.gov/safewater/contaminants/dw contamfs/24-d.htm

U.S. Environmental Protection Agency, 2006b, Consumer factsheet on carbofuran, accessed on April 05, 2006, at http://www.epa.gov/safewater/contaminants/dw_contamfs, carbofur.html 
U.S. Environmental Protection Agency, 2004a, RED FACTS MCPA, accessed on April 05, 2006, at http://www.epa. gov/oppsrrdl/REDs/factsheets/mcpa_red_fs.pd]

U.S. Environmental Protection Agency, 2004b, Carbaryl IRED Facts, accessed on April 05, 2006, at http://www.epa.gov/ oppsrrd1/REDs/factsheets/carbaryl_factsheet.pdf

U.S. Environmental Protection Agency, 2002a, List of National Secondary Drinking Water Regulations, accessed on March 14, 2005, at http://www.epa.gov/safewater/mcl. html\#sec

U.S. Environmental Protection Agency, 2002b, Method 1603-Escherichia coli (E. coli) in Water by membrane filtration using modified membrane-thermotolerant $E s c h$ erichia coli agar (Modified mTEC): Washington, D.C., EPA-821-R-02-023, September 2002.

U.S. Environmental Protection Agency, 2001, Benomyl RED Facts: Benomyl Fact Sheet, EPA-738-F-02-001.

U.S. Environmental Protection Agency, 1996, RED FACTS Norflurazon, accessed on April 05, 2006, at http://www.epa. gov/oppsrrd1/REDs/factsheets/0229fact.pd]

U.S. Environmental Protection Agency, 1994, RED FACTS Metalaxyl, accessed on April 05, 2006, at http://www.epa. gov/oppsrrd1/REDs/factsheets/0081fact.pd]
Wagner, R.J., Boulger, R.W., Jr., Oblinger, C.J., and Smith, B.A., 2006, Guidelines and standard procedures for continuous water-quality monitors-Station operation, record computation, and data reporting: U.S. Geological Survey Techniques and Methods 1-D3, 51 p., plus 8 attachments, accessed on April 10, 2006, at http://pubs.water.usgs. gov/tmld3

Wagner, R.J., Frans, L.M., and Huffman, R.L., 2006, Occurrence, distribution, and transport of pesticides in agricultural irrigation return flow from four drainage basins in the Columbia Basin Project, Washington, 2002-2004, and comparison with historical data: U.S. Geological Survey Scientific Investigations Report 2006-5005, 54 p.

Waldron, M.C., and Bent, G.C., 2001, Factors affecting reservoir and stream-water quality in the Cambridge, Massachusetts, drinking-water source area and implications for source-water protection: U.S. Geological Survey WaterResources Investigations Report 00-4262, 89 p.

Wershaw, R.L., Fishman, M.J., Grabbe, R.R., and Lowe, L.E., eds., 1987, Methods for the determination of organic substances in water and fluvial sediments: U.S. Geological Survey Techniques of Water-Resources Investigations, book 5 , chap. A3, $80 \mathrm{p}$.

Wilde, F.D., Radtke, D.B., Gibs, Jacob, and Iwatsubo, R.T., eds., September 1999, Collection of water samples: U.S. Geological Survey Techniques of Water-Resources Investigations, book 9, chap. A4, accessed on November 2, 2006, at http://pubs.water.usgs.gov/twri9A4/ 



\section{Tables 8-16}


Table 8. Daily, monthly, and annual statistics for discharge for U.S. Geological Survey stations in the drinking-water source area for Cambridge, Massachusetts, for water year 2005.

[Units are in millions of gallons. e, estimated; —, no data; $\mathrm{Mgal} / \mathrm{d} / \mathrm{mi}^{2}$, million gallons per day per square mile; \%, percent]

\begin{tabular}{|c|c|c|c|c|c|c|c|}
\hline $\begin{array}{l}\text { Month and } \\
\text { date }\end{array}$ & $\begin{array}{c}\text { Cambridge } \\
\text { Reservoir, } \\
\text { unnamed } \\
\text { tributary 2, } \\
\text { near Lexington } \\
\text { (01104415) } \\
\end{array}$ & $\begin{array}{c}\text { Cambridge } \\
\text { Reservoir near } \\
\text { Kendal Green } \\
\text { (01104430) }\end{array}$ & $\begin{array}{l}\text { Hobbs Brook, } \\
\text { unnamed } \\
\text { tributary 1, near } \\
\text { Kendal Green } \\
\text { (01104433) }\end{array}$ & $\begin{array}{l}\text { Stony Brook, } \\
\text { unnamed } \\
\text { tributary 1, } \\
\text { near Waltham } \\
\text { (01104455) }\end{array}$ & $\begin{array}{l}\text { Stony Brook } \\
\text { at Route } 20 \\
\text { at Waltham } \\
(01104460)\end{array}$ & $\begin{array}{c}\text { Stony Brook } \\
\text { Reservoir, } \\
\text { unnamed } \\
\text { tributary 1, } \\
\text { near Weston } \\
\text { (01104475) }\end{array}$ & $\begin{array}{l}\text { Stony Brook } \\
\text { Reservoir } \\
\text { at dam near } \\
\text { Waltham } \\
\text { (01104480) }\end{array}$ \\
\hline \multicolumn{8}{|c|}{ October } \\
\hline 3 & .33 & .61 & .26 & .22 & 15 & .75 & e. 20 \\
\hline 4 & .26 & .53 & .17 & .19 & 15 & .71 & e. 41 \\
\hline 5 & .22 & .54 & .15 & .16 & 14 & .58 & .16 \\
\hline 6 & .19 & .54 & .14 & .15 & 12 & .54 & .16 \\
\hline 7 & .17 & .54 & .13 & .13 & 11 & .51 & .16 \\
\hline 11 & .14 & 3.5 & .10 & .13 & 13 & .34 & .16 \\
\hline 12 & .16 & .75 & .13 & .14 & 8.8 & .33 & .22 \\
\hline 13 & .12 & .75 & .10 & .13 & 8.0 & .33 & .22 \\
\hline 14 & .12 & 3.6 & .10 & .14 & 8.6 & .35 & .22 \\
\hline 15 & .40 & 5.4 & .37 & .64 & 13 & .41 & .26 \\
\hline 16 & 1.6 & 5.5 & 1.1 & 1.3 & 26 & 1.2 & .43 \\
\hline 17 & .26 & 5.4 & .17 & .15 & 20 & .64 & .34 \\
\hline 18 & .19 & 2.9 & .14 & .13 & 16 & .52 & .26 \\
\hline 19 & .69 & .88 & .87 & .91 & 16 & .76 & .55 \\
\hline 20 & .31 & .88 & .24 & .18 & 15 & .72 & .55 \\
\hline 26 & .14 & 4.4 & .12 & .13 & 12 & .45 & .54 \\
\hline 27 & .13 & 4.5 & .12 & .12 & 11 & .42 & .49 \\
\hline 28 & .11 & 4.4 & .11 & .12 & 11 & .37 & .43 \\
\hline 29 & .13 & 4.3 & .11 & .13 & 11 & .39 & .43 \\
\hline 30 & .14 & 7.4 & .14 & .14 & 12 & .41 & .55 \\
\hline 31 & .13 & 9.2 & .11 & .15 & 16 & .38 & .48 \\
\hline Total & 8.13 & 95.77 & 6.48 & 7.16 & 427.4 & 17.01 & 10.71 \\
\hline Mean & .26 & 3.09 & .21 & .23 & 13.8 & .55 & .35 \\
\hline Maximum & 1.6 & 9.2 & 1.1 & 1.3 & 26 & 1.2 & .55 \\
\hline Minimum & .11 & .53 & .10 & .12 & 8.0 & .33 & .13 \\
\hline Median & .18 & 3.0 & .13 & .14 & 13 & .49 & .34 \\
\hline $\mathrm{Mgal} / \mathrm{d} / \mathrm{mi}^{2}$ & .64 & - & .58 & .48 & - & .65 & - \\
\hline Inches & 1.13 & - & 1.03 & .86 & - & 1.15 & - \\
\hline
\end{tabular}


Table 8. Daily, monthly, and annual statistics for discharge for U.S. Geological Survey stations in the drinking-water source area for Cambridge, Massachusetts, for water year 2005.-Continued

[Units are in millions of gallons. e, estimated; —, no data; $\mathrm{Mgal} / \mathrm{d} / \mathrm{mi}^{2}$, million gallons per day per square mile; \%, percent]

\begin{tabular}{|c|c|c|c|c|c|c|c|}
\hline $\begin{array}{l}\text { Month and } \\
\text { date }\end{array}$ & $\begin{array}{c}\text { Cambridge } \\
\text { Reservoir, } \\
\text { unnamed } \\
\text { tributary 2, } \\
\text { near Lexington } \\
\text { (01104415) }\end{array}$ & $\begin{array}{c}\text { Cambridge } \\
\text { Reservoir near } \\
\text { Kendal Green } \\
\text { (01104430) }\end{array}$ & $\begin{array}{l}\text { Hobbs Brook, } \\
\text { unnamed } \\
\text { tributary 1, near } \\
\text { Kendal Green } \\
\text { (01104433) }\end{array}$ & $\begin{array}{l}\text { Stony Brook, } \\
\text { unnamed } \\
\text { tributary 1, } \\
\text { near Waltham } \\
\text { (01104455) }\end{array}$ & $\begin{array}{c}\text { Stony Brook } \\
\text { at Route } 20 \\
\text { at Waltham } \\
(01104460)\end{array}$ & $\begin{array}{c}\text { Stony Brook } \\
\text { Reservoir, } \\
\text { unnamed } \\
\text { tributary 1, } \\
\text { near Weston } \\
\text { (01104475) }\end{array}$ & $\begin{array}{c}\text { Stony Brook } \\
\text { Reservoir } \\
\text { at dam near } \\
\text { Waltham } \\
\text { (01104480) }\end{array}$ \\
\hline \multicolumn{8}{|c|}{ November } \\
\hline 1 & 0.11 & 9.2 & 0.09 & 0.16 & 15 & 0.31 & 0.55 \\
\hline 4 & .65 & 9.5 & .27 & 1.0 & 16 & .43 & .55 \\
\hline 5 & .69 & 9.6 & .56 & .34 & 24 & .71 & .55 \\
\hline 6 & .19 & 9.8 & .12 & .21 & 20 & .51 & 1.5 \\
\hline 7 & .15 & 9.8 & .10 & .19 & 19 & .47 & 1.5 \\
\hline 11 & .11 & 9.5 & .08 & .16 & 16 & .38 & 1.6 \\
\hline 12 & .17 & 9.5 & .12 & .33 & 16 & .38 & 1.8 \\
\hline 13 & .28 & 9.4 & .17 & .35 & 18 & .44 & 1.9 \\
\hline 14 & .16 & 9.4 & .12 & .17 & 17 & .41 & 2.1 \\
\hline 15 & .23 & 9.4 & .15 & .26 & 17 & .43 & 2.3 \\
\hline 16 & .22 & 9.4 & .14 & .23 & 17 & .45 & 2.3 \\
\hline 17 & .21 & 8.2 & .14 & .24 & 17 & .47 & 2.3 \\
\hline 18 & .22 & 6.2 & .12 & .18 & 16 & .47 & 2.3 \\
\hline 19 & .17 & 3.9 & .08 & .16 & 13 & e. 50 & 2.3 \\
\hline 20 & .17 & 3.0 & .10 & .23 & 11 & e. 47 & 2.5 \\
\hline 27 & .18 & .32 & .09 & .13 & 11 & .53 & 3.0 \\
\hline 28 & 1.9 & .30 & .60 & 2.3 & 18 & 1.1 & 3.0 \\
\hline 29 & .67 & .33 & .96 & .42 & 31 & 1.9 & .78 \\
\hline 30 & .41 & .32 & .21 & .31 & 23 & 1.2 & 7.1 \\
\hline Total & 9.22 & 180.82 & 6.05 & 9.58 & 480.9 & 16.65 & 61.37 \\
\hline Mean & .31 & 6.03 & .20 & .32 & 16 & .56 & 2.05 \\
\hline Maximum & 1.9 & 9.8 & .96 & 2.3 & 31 & 1.9 & 7.1 \\
\hline Minimum & .10 & .30 & .08 & .10 & 9.4 & .31 & .49 \\
\hline Median & .18 & 9.2 & .12 & .19 & 16 & .47 & 2.2 \\
\hline $\mathrm{Mgal} / \mathrm{d} / \mathrm{mi}^{2}$ & .75 & - & .56 & .67 & - & .65 & - \\
\hline Inches & 1.3 & - & .97 & 1.15 & - & 1.13 & - \\
\hline
\end{tabular}


Table 8. Daily, monthly, and annual statistics for discharge for U.S. Geological Survey stations in the drinking-water source area for Cambridge, Massachusetts, for water year 2005.-Continued

[Units are in millions of gallons. e, estimated; —, no data; $\mathrm{Mgal} / \mathrm{d} / \mathrm{mi}^{2}$, million gallons per day per square mile; \%, percent]

\begin{tabular}{|c|c|c|c|c|c|c|c|}
\hline $\begin{array}{l}\text { Month and } \\
\text { date }\end{array}$ & $\begin{array}{c}\text { Cambridge } \\
\text { Reservoir, } \\
\text { unnamed } \\
\text { tributary 2, } \\
\text { near Lexington } \\
\text { (01104415) } \\
\end{array}$ & $\begin{array}{c}\text { Cambridge } \\
\text { Reservoir near } \\
\text { Kendal Green } \\
(01104430)\end{array}$ & $\begin{array}{l}\text { Hobbs Brook, } \\
\text { unnamed } \\
\text { tributary 1, near } \\
\text { Kendal Green } \\
\text { (01104433) }\end{array}$ & $\begin{array}{c}\text { Stony Brook, } \\
\text { unnamed } \\
\text { tributary 1, } \\
\text { near Waltham } \\
\text { (01104455) }\end{array}$ & $\begin{array}{l}\text { Stony Brook } \\
\text { at Route } 20 \\
\text { at Waltham } \\
(01104460)\end{array}$ & $\begin{array}{c}\text { Stony Brook } \\
\text { Reservoir, } \\
\text { unnamed } \\
\text { tributary 1, } \\
\text { near Weston } \\
\text { (01104475) }\end{array}$ & $\begin{array}{c}\text { Stony Brook } \\
\text { Reservoir } \\
\text { at dam near } \\
\text { Waltham } \\
\text { (01104480) }\end{array}$ \\
\hline \multicolumn{8}{|c|}{ December } \\
\hline 1 & 1.5 & 0.29 & 0.84 & 1.6 & 28 & 1.4 & 38 \\
\hline 4 & .38 & .23 & .16 & .36 & 20 & .95 & .90 \\
\hline 5 & .35 & .23 & .14 & .33 & 18 & .88 & 24 \\
\hline 6 & .31 & .23 & .12 & .32 & 15 & .80 & 24 \\
\hline 7 & 2.3 & .27 & .81 & 2.6 & 23 & 1.4 & 11 \\
\hline 11 & 1.9 & .45 & .98 & 1.8 & 48 & 2.3 & 35 \\
\hline 12 & .77 & .49 & .32 & 1.0 & 41 & 1.8 & 36 \\
\hline 13 & .64 & .52 & .24 & .89 & 33 & 1.5 & 36 \\
\hline 14 & .54 & .52 & .20 & .78 & 29 & 1.3 & 20 \\
\hline 15 & .46 & .52 & .15 & .71 & 24 & 1.2 & 20 \\
\hline 16 & .44 & .54 & .13 & .70 & 21 & 1.1 & 20 \\
\hline 17 & .43 & .54 & .12 & .65 & 20 & 1.1 & 20 \\
\hline 18 & .38 & .52 & .10 & .56 & 19 & .98 & 9.7 \\
\hline 19 & .39 & .54 & .11 & .57 & 18 & .95 & .22 \\
\hline 20 & .42 & .56 & .19 & .60 & 19 & 1.1 & .26 \\
\hline 27 & .49 & .85 & e. 15 & e.56 & 25 & 1.4 & 25 \\
\hline 28 & .45 & 4.1 & e. 15 & e.56 & 23 & $\mathrm{e} 1.2$ & 48 \\
\hline 29 & .44 & 9.9 & e. 15 & e.56 & 29 & $\mathrm{e} 1.2$ & 48 \\
\hline 30 & .41 & 14 & e. 15 & e. 55 & 33 & e.89 & 26 \\
\hline 31 & .51 & 15 & e. 27 & e.71 & 35 & $\mathrm{e} 1.0$ & 21 \\
\hline Total & 23.18 & 55.98 & e10.05 & e26.82 & 861 & e41.26 & 621.4 \\
\hline Mean & .75 & 1.81 & e.32 & e.87 & 27.8 & e1.33 & 20 \\
\hline Maximum & 2.6 & 15 & 1.1 & 2.7 & 49 & 2.4 & 48 \\
\hline Minimum & .31 & .23 & .10 & .32 & 15 & e.80 & .22 \\
\hline Median & .49 & .52 & e.19 & e.65 & 25 & e1.2 & 20 \\
\hline Mgal/d/mi ${ }^{2}$ & 1.82 & - & e.90 & e1.8 & - & e1.57 & - \\
\hline Inches & 3.24 & - & e1.6 & e3.2 & - & e2.79 & - \\
\hline
\end{tabular}


Table 8. Daily, monthly, and annual statistics for discharge for U.S. Geological Survey stations in the drinking-water source area for Cambridge, Massachusetts, for water year 2005.-Continued

[Units are in millions of gallons. e, estimated; —, no data; $\mathrm{Mgal} / \mathrm{d} / \mathrm{mi}^{2}$, million gallons per day per square mile; \%, percent]

\begin{tabular}{|c|c|c|c|c|c|c|c|}
\hline $\begin{array}{l}\text { Month and } \\
\text { date }\end{array}$ & $\begin{array}{c}\text { Cambridge } \\
\text { Reservoir, } \\
\text { unnamed } \\
\text { tributary 2, } \\
\text { near Lexington } \\
\text { (01104415) }\end{array}$ & $\begin{array}{c}\text { Cambridge } \\
\text { Reservoir near } \\
\text { Kendal Green } \\
\text { (01104430) }\end{array}$ & $\begin{array}{l}\text { Hobbs Brook, } \\
\text { unnamed } \\
\text { tributary 1, near } \\
\text { Kendal Green } \\
\text { (01104433) }\end{array}$ & $\begin{array}{l}\text { Stony Brook, } \\
\text { unnamed } \\
\text { tributary 1, } \\
\text { near Waltham } \\
\text { (01104455) }\end{array}$ & $\begin{array}{l}\text { Stony Brook } \\
\text { at Route } 20 \\
\text { at Waltham } \\
(01104460)\end{array}$ & $\begin{array}{c}\text { Stony Brook } \\
\text { Reservoir, } \\
\text { unnamed } \\
\text { tributary 1, } \\
\text { near Weston } \\
\text { (01104475) }\end{array}$ & $\begin{array}{c}\text { Stony Brook } \\
\text { Reservoir } \\
\text { at dam near } \\
\text { Waltham } \\
\text { (01104480) }\end{array}$ \\
\hline \multicolumn{8}{|c|}{ January } \\
\hline 1 & 0.73 & 15 & $\mathrm{e} 0.34$ & $\mathrm{e} 0.97$ & 38 & $\mathrm{e} 1.3$ & $\mathrm{e} 23$ \\
\hline 4 & 1.5 & 15 & .83 & 1.7 & 52 & 2.0 & 37 \\
\hline 5 & .73 & 15 & .29 & .98 & 51 & 1.7 & 43 \\
\hline 6 & .88 & 15 & .40 & 1.4 & 48 & 1.7 & 43 \\
\hline 7 & .87 & 15 & .62 & 1.2 & 49 & 1.7 & 43 \\
\hline 11 & .55 & 15 & .18 & .83 & 41 & 1.4 & 28 \\
\hline 12 & 1.0 & 15 & .56 & 1.5 & 43 & 1.6 & 36 \\
\hline 13 & 1.0 & 15 & .64 & 1.4 & 48 & 1.8 & 42 \\
\hline 14 & 5.3 & 15 & 1.5 & 3.9 & 81 & 4.1 & 43 \\
\hline 15 & 1.5 & 15 & .94 & 2.1 & 106 & 3.4 & 52 \\
\hline 16 & 1.1 & 15 & .36 & 1.8 & 84 & 2.8 & 69 \\
\hline 17 & .92 & 15 & .28 & 1.6 & 70 & 2.3 & 60 \\
\hline 18 & .72 & 15 & .19 & $\mathrm{e} 1.3$ & 58 & 1.9 & 51 \\
\hline 19 & .65 & 15 & .17 & $\mathrm{e} 1.2$ & 50 & 1.7 & 44 \\
\hline 20 & .65 & 15 & .15 & 1.2 & 49 & 1.7 & 44 \\
\hline 27 & .40 & 14 & .11 & e. 65 & 40 & 1.1 & 23 \\
\hline 28 & .38 & 14 & .09 & e.64 & 38 & .99 & 23 \\
\hline 29 & .38 & 14 & .09 & e.64 & 37 & .96 & 23 \\
\hline 30 & .37 & 14 & .09 & .58 & 36 & .95 & 33 \\
\hline 31 & .34 & 14 & .07 & .53 & 35 & .91 & 32 \\
\hline Total & 26.11 & 460 & e10.05 & e35.84 & 1,524 & e51.61 & e1,190 \\
\hline Mean & .84 & 14.8 & e.32 & e1.2 & 49 & e1.7 & e38.4 \\
\hline Maximum & 5.3 & 15 & 1.5 & 3.9 & 106 & 4.1 & 69 \\
\hline Minimum & .34 & 14 & e.07 & e.53 & 35 & e.91 & e23 \\
\hline Median & .65 & 15 & .23 & e.97 & 43 & e1.5 & e42 \\
\hline $\mathrm{Mgal} / \mathrm{d} / \mathbf{m i}^{2}$ & 2.05 & - & e.90 & e2.41 & - & e1.96 & - \\
\hline Inches & 3.67 & - & e1.62 & e4.29 & - & e3.49 & - \\
\hline
\end{tabular}


Table 8. Daily, monthly, and annual statistics for discharge for U.S. Geological Survey stations in the drinking-water source area for Cambridge, Massachusetts, for water year 2005.-Continued

[Units are in millions of gallons. e, estimated; —, no data; $\mathrm{Mgal} / \mathrm{d} / \mathrm{mi}^{2}$, million gallons per day per square mile; \%, percent]

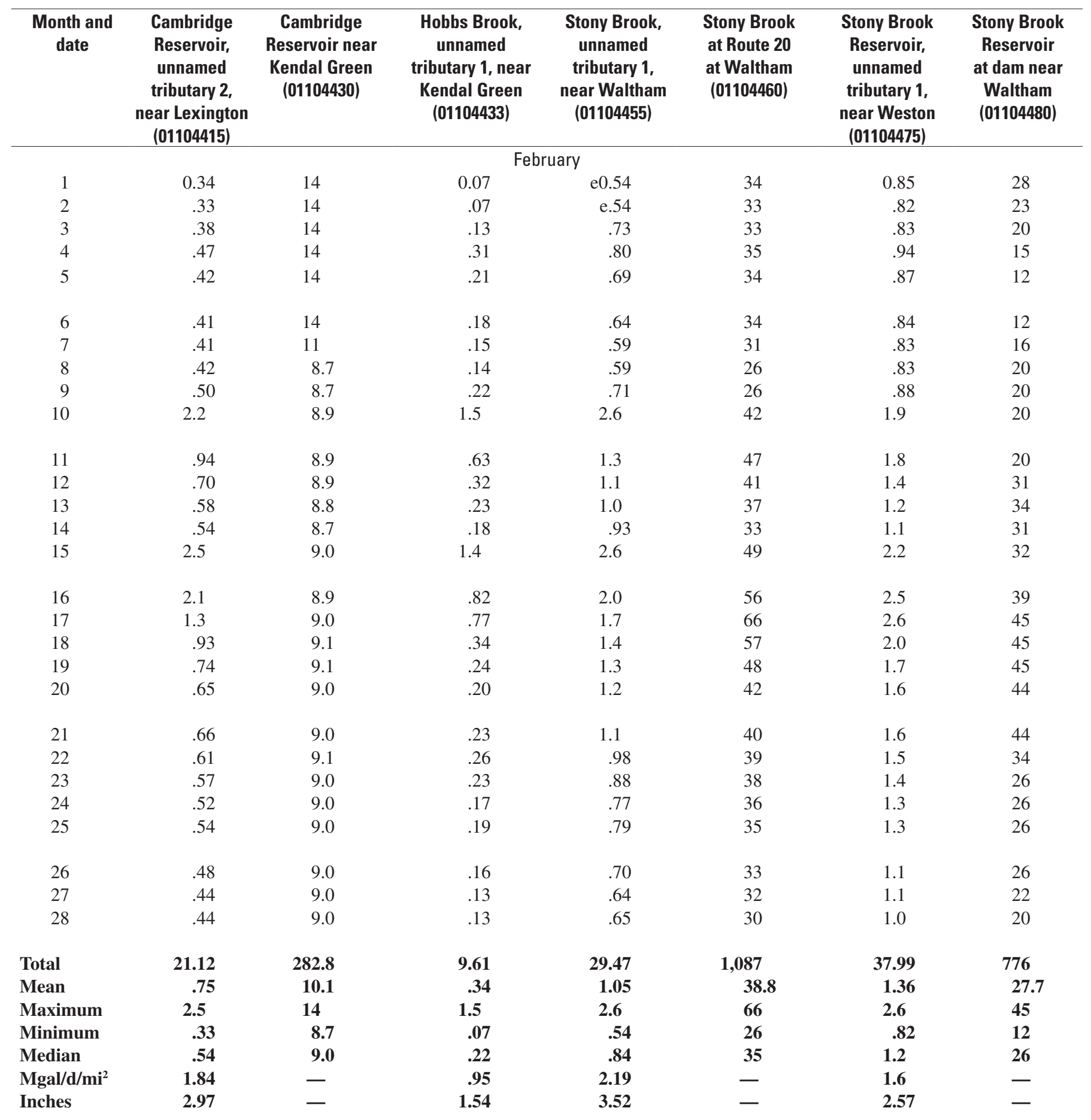


Table 8. Daily, monthly, and annual statistics for discharge for U.S. Geological Survey stations in the drinking-water source area for Cambridge, Massachusetts, for water year 2005.-Continued

[Units are in millions of gallons. e, estimated; —, no data; $\mathrm{Mgal} / \mathrm{d} / \mathrm{mi}^{2}$, million gallons per day per square mile; \%, percent]

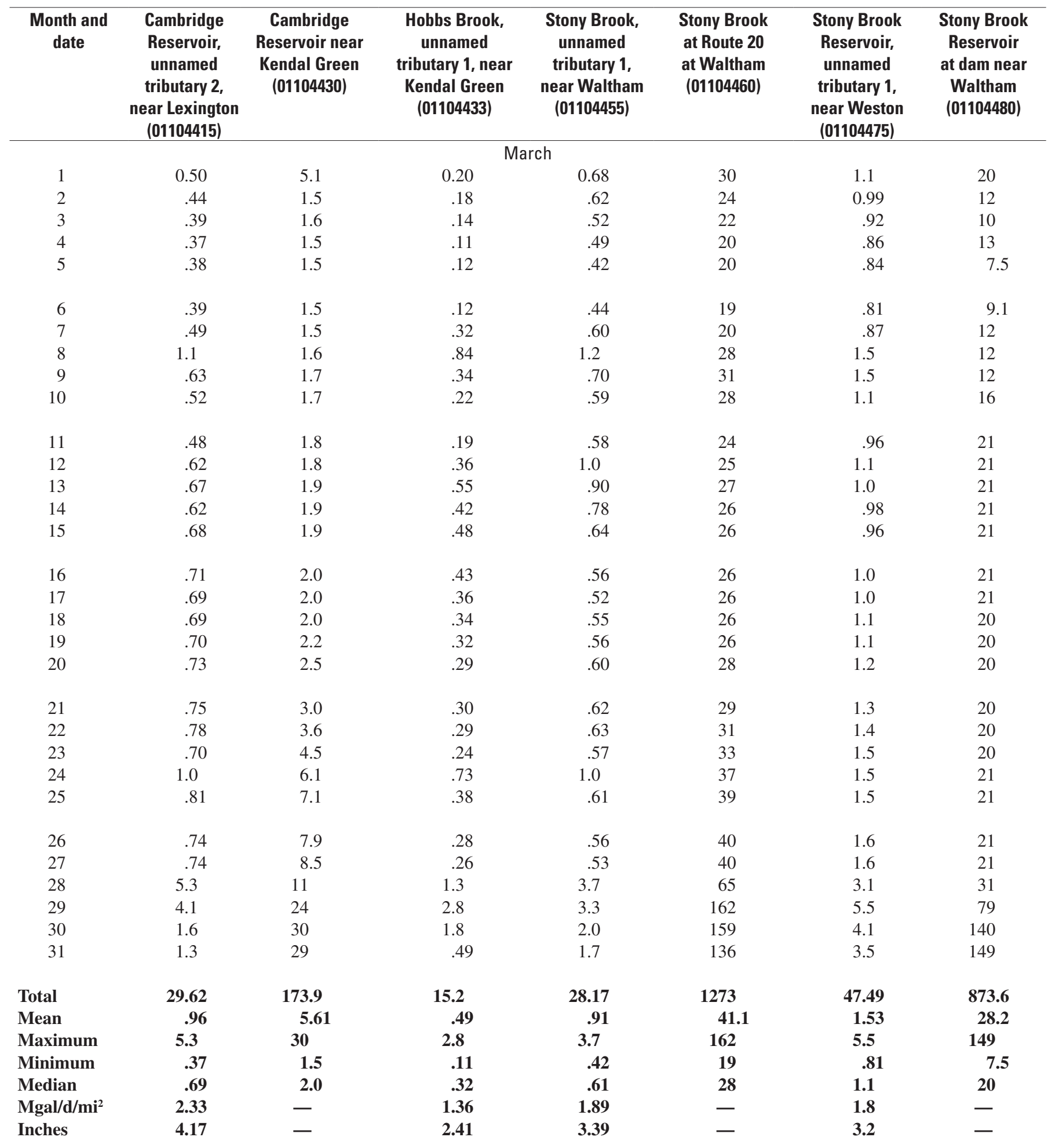


Table 8. Daily, monthly, and annual statistics for discharge for U.S. Geological Survey stations in the drinking-water source area for Cambridge, Massachusetts, for water year 2005.-Continued

[Units are in millions of gallons. e, estimated; —, no data; $\mathrm{Mgal} / \mathrm{d} / \mathrm{mi}^{2}$, million gallons per day per square mile; \%, percent]

\begin{tabular}{|c|c|c|c|c|c|c|c|}
\hline $\begin{array}{l}\text { Month and } \\
\text { date }\end{array}$ & $\begin{array}{c}\text { Cambridge } \\
\text { Reservoir, } \\
\text { unnamed } \\
\text { tributary 2, } \\
\text { near Lexington } \\
\text { (01104415) }\end{array}$ & $\begin{array}{c}\text { Cambridge } \\
\text { Reservoir near } \\
\text { Kendal Green } \\
\text { (01104430) }\end{array}$ & $\begin{array}{l}\text { Hobbs Brook, } \\
\text { unnamed } \\
\text { tributary 1, near } \\
\text { Kendal Green } \\
\text { (01104433) }\end{array}$ & $\begin{array}{l}\text { Stony Brook, } \\
\text { unnamed } \\
\text { tributary 1, } \\
\text { near Waltham } \\
\text { (01104455) }\end{array}$ & $\begin{array}{l}\text { Stony Brook } \\
\text { at Route } 20 \\
\text { at Waltham } \\
(01104460)\end{array}$ & $\begin{array}{c}\text { Stony Brook } \\
\text { Reservoir, } \\
\text { unnamed } \\
\text { tributary 1, } \\
\text { near Weston } \\
\text { (01104475) }\end{array}$ & $\begin{array}{l}\text { Stony Brook } \\
\text { Reservoir } \\
\text { at dam near } \\
\text { Waltham } \\
\text { (01104480) }\end{array}$ \\
\hline \multicolumn{8}{|c|}{ April } \\
\hline 1 & 1.1 & 26 & 0.39 & 1.5 & 114 & 3.1 & 194 \\
\hline 4 & 1.4 & 37 & 1.2 & 1.9 & 160 & 3.9 & 144 \\
\hline 5 & 1.1 & 29 & .44 & 1.7 & 130 & 3.2 & 100 \\
\hline 6 & .95 & 24 & .36 & 1.5 & 100 & 2.6 & 82 \\
\hline 7 & .88 & 22 & .31 & 1.4 & 86 & 2.2 & 67 \\
\hline 11 & .61 & 19 & .20 & 1.0 & 60 & 1.6 & 39 \\
\hline 12 & .55 & 17 & .19 & .98 & 53 & 1.5 & 30 \\
\hline 13 & .54 & 16 & .19 & .96 & 50 & 1.4 & 30 \\
\hline 14 & .50 & 15 & .16 & .93 & 46 & 1.3 & 30 \\
\hline 15 & .45 & 13 & .14 & .89 & 42 & 1.2 & 30 \\
\hline 16 & .44 & 12 & .13 & .88 & 39 & 1.1 & 19 \\
\hline 17 & .43 & 11 & .11 & .88 & 36 & 1.0 & 12 \\
\hline 18 & .39 & 11 & .11 & .88 & 34 & .95 & 12 \\
\hline 19 & .37 & 9.3 & .10 & .87 & 31 & .90 & 12 \\
\hline 20 & .36 & 8.9 & .10 & .91 & 29 & .81 & 12 \\
\hline 27 & .91 & 12 & .87 & 1.4 & 40 & 1.2 & 40 \\
\hline 28 & .67 & 13 & .59 & .82 & 45 & 1.5 & 39 \\
\hline 29 & .49 & 12 & .28 & .71 & 40 & 1.2 & 39 \\
\hline 30 & .88 & 12 & .62 & 1.4 & 37 & 1.2 & 39 \\
\hline Total & 27.07 & 514.4 & 14.65 & 39.51 & 1,933 & 54.55 & 1,621 \\
\hline Mean & .90 & 17.1 & .49 & 1.32 & 64.4 & 1.82 & 54 \\
\hline Maximum & 3.8 & 37 & 2.0 & 4.2 & 175 & 5.2 & 194 \\
\hline Minimum & .33 & 8.7 & .10 & .66 & 27 & .81 & 12 \\
\hline Median & .72 & 13 & .29 & 1.0 & 46 & 1.4 & 39 \\
\hline Mgal/d//mi ${ }^{2}$ & 2.2 & - & 1.36 & 2.74 & - & 2.14 & - \\
\hline Inches & 3.81 & - & 2.34 & 4.74 & - & 3.69 & - \\
\hline
\end{tabular}


Table 8. Daily, monthly, and annual statistics for discharge for U.S. Geological Survey stations in the drinking-water source area for Cambridge, Massachusetts, for water year 2005.-Continued

[Units are in millions of gallons. e, estimated; —, no data; $\mathrm{Mgal} / \mathrm{d} / \mathrm{mi}^{2}$, million gallons per day per square mile; \%, percent]

\begin{tabular}{|c|c|c|c|c|c|c|c|}
\hline $\begin{array}{l}\text { Month and } \\
\text { date }\end{array}$ & $\begin{array}{c}\text { Cambridge } \\
\text { Reservoir, } \\
\text { unnamed } \\
\text { tributary 2, } \\
\text { near Lexington } \\
\text { (01104415) }\end{array}$ & $\begin{array}{c}\text { Cambridge } \\
\text { Reservoir near } \\
\text { Kendal Green } \\
(01104430)\end{array}$ & $\begin{array}{l}\text { Hobbs Brook, } \\
\text { unnamed } \\
\text { tributary 1, near } \\
\text { Kendal Green } \\
\text { (01104433) }\end{array}$ & $\begin{array}{c}\text { Stony Brook, } \\
\text { unnamed } \\
\text { tributary 1, } \\
\text { near Waltham } \\
\text { (01104455) }\end{array}$ & $\begin{array}{c}\text { Stony Brook } \\
\text { at Route } 20 \\
\text { at Waltham } \\
(01104460)\end{array}$ & $\begin{array}{c}\text { Stony Brook } \\
\text { Reservoir, } \\
\text { unnamed } \\
\text { tributary 1, } \\
\text { near Weston } \\
\text { (01104475) }\end{array}$ & $\begin{array}{c}\text { Stony Brook } \\
\text { Reservoir } \\
\text { at dam near } \\
\text { Waltham } \\
\text { (01104480) }\end{array}$ \\
\hline \multicolumn{8}{|c|}{ May } \\
\hline 1 & 0.84 & 13 & 1.2 & 1.1 & 45 & 1.5 & 39 \\
\hline 4 & .41 & 12 & .27 & .73 & 34 & .99 & 21 \\
\hline 5 & .37 & 11 & .22 & .71 & 32 & .92 & 21 \\
\hline 6 & .36 & 9.9 & .20 & .69 & 29 & .87 & 21 \\
\hline 7 & 1.4 & 11 & 1.6 & 2.2 & 37 & 1.5 & 21 \\
\hline 11 & .42 & 11 & .18 & .76 & 33 & .94 & 22 \\
\hline 12 & .37 & 11 & .15 & .74 & 29 & .85 & 12 \\
\hline 13 & .34 & 9.5 & .12 & .73 & 26 & .79 & .32 \\
\hline 14 & .34 & 8.9 & .12 & .72 & 25 & .77 & .34 \\
\hline 15 & .33 & 8.5 & .11 & .72 & 23 & .73 & 2.6 \\
\hline 16 & .47 & 8.6 & .62 & 1.1 & 28 & .89 & 11 \\
\hline 17 & .32 & 8.1 & .14 & .58 & 24 & .80 & 13 \\
\hline 18 & .36 & 8.0 & .11 & .56 & 21 & .60 & 10 \\
\hline 19 & .31 & 7.8 & .10 & .56 & 21 & .68 & 7.3 \\
\hline 20 & .28 & 7.4 & .08 & .53 & 19 & .68 & 19 \\
\hline 27 & 1.2 & 22 & .62 & 1.4 & 87 & 2.1 & 71 \\
\hline 28 & .89 & 21 & .31 & 1.3 & 64 & 1.7 & 53 \\
\hline 29 & .79 & 19 & .38 & 1.5 & 53 & 1.5 & 39 \\
\hline 30 & .68 & 17 & .24 & 1.1 & 48 & 1.4 & 39 \\
\hline 31 & .61 & 16 & .22 & 1.0 & 43 & 1.3 & 39 \\
\hline Total & 23.34 & 371.1 & 14.97 & 33.02 & 1,176 & 36.1 & 864.56 \\
\hline Mean & .75 & 12 & .48 & 1.07 & 37.9 & 1.16 & 27.9 \\
\hline Maximum & 3.5 & 22 & 2.0 & 3.1 & 101 & 3.3 & 94 \\
\hline Minimum & .28 & 7.2 & .08 & .53 & 18 & .60 & .32 \\
\hline Median & .47 & 11 & .24 & .81 & 34 & .99 & 22 \\
\hline $\mathrm{Mgal} / \mathrm{d} / \mathrm{mi}^{2}$ & 1.84 & - & 1.34 & 2.22 & - & 1.37 & - \\
\hline Inches & 3.27 & - & 2.42 & 3.96 & - & 2.44 & - \\
\hline
\end{tabular}


Table 8. Daily, monthly, and annual statistics for discharge for U.S. Geological Survey stations in the drinking-water source area for Cambridge, Massachusetts, for water year 2005.-Continued

[Units are in millions of gallons. e, estimated; —, no data; $\mathrm{Mgal} / \mathrm{d} / \mathrm{mi}^{2}$, million gallons per day per square mile; \%, percent]

\begin{tabular}{|c|c|c|c|c|c|c|c|}
\hline $\begin{array}{l}\text { Month and } \\
\text { date }\end{array}$ & $\begin{array}{c}\text { Cambridge } \\
\text { Reservoir, } \\
\text { unnamed } \\
\text { tributary 2, } \\
\text { near Lexington } \\
\text { (01104415) }\end{array}$ & $\begin{array}{c}\text { Cambridge } \\
\text { Reservoir near } \\
\text { Kendal Green } \\
\text { (01104430) }\end{array}$ & $\begin{array}{l}\text { Hobbs Brook, } \\
\text { unnamed } \\
\text { tributary 1, near } \\
\text { Kendal Green } \\
\text { (01104433) }\end{array}$ & $\begin{array}{c}\text { Stony Brook, } \\
\text { unnamed } \\
\text { tributary 1, } \\
\text { near Waltham } \\
\text { (01104455) }\end{array}$ & $\begin{array}{c}\text { Stony Brook } \\
\text { at Route } 20 \\
\text { at Waltham } \\
(01104460)\end{array}$ & $\begin{array}{c}\text { Stony Brook } \\
\text { Reservoir, } \\
\text { unnamed } \\
\text { tributary 1, } \\
\text { near Weston } \\
\text { (01104475) }\end{array}$ & $\begin{array}{l}\text { Stony Brook } \\
\text { Reservoir } \\
\text { at dam near } \\
\text { Waltham } \\
\text { (01104480) }\end{array}$ \\
\hline \multicolumn{8}{|c|}{ June } \\
\hline 1 & 0.55 & 15 & 0.24 & 0.93 & 39 & 1.2 & 39 \\
\hline 4 & .43 & 12 & .09 & .75 & 29 & .84 & 5.1 \\
\hline 5 & .39 & 11 & .07 & .70 & 26 & .79 & .24 \\
\hline 6 & .38 & 10 & .05 & .66 & 23 & .72 & .25 \\
\hline 7 & .35 & 9.3 & .06 & .63 & 22 & .66 & .28 \\
\hline 11 & .39 & 11 & .12 & .53 & 30 & .83 & 44 \\
\hline 12 & .36 & 9.9 & .11 & .49 & 26 & .72 & 34 \\
\hline 13 & .34 & 9.1 & .10 & .47 & 22 & .65 & 13 \\
\hline 14 & .39 & 8.9 & .41 & .51 & 21 & .64 & 13 \\
\hline 15 & .56 & 8.9 & .27 & .61 & 20 & .66 & 6.3 \\
\hline 16 & .48 & 8.5 & .18 & .72 & 19 & .70 & .21 \\
\hline 17 & .51 & 8.6 & .32 & .54 & 21 & .85 & .23 \\
\hline 18 & .34 & 8.2 & .12 & .39 & 19 & .70 & .21 \\
\hline 19 & .30 & 7.8 & .11 & .36 & 19 & .66 & .22 \\
\hline 20 & .27 & 7.4 & .10 & .33 & 17 & .63 & .24 \\
\hline 27 & .10 & 6.3 & .08 & .25 & 12 & e. 26 & .43 \\
\hline 28 & .11 & 6.2 & .10 & .25 & 11 & e. 26 & .39 \\
\hline 29 & .11 & 7.8 & .07 & .24 & 11 & e. 26 & .44 \\
\hline 30 & .10 & 10 & .07 & .23 & 13 & e. 26 & .49 \\
\hline Total & 11.98 & 274.7 & 4.83 & 16.39 & 646 & e19.47 & 218.08 \\
\hline Mean & .40 & 9.16 & .16 & .55 & 21.5 & e.65 & 7.27 \\
\hline Maximum & 1.5 & 15 & 1.0 & 1.7 & 42 & 1.6 & 44 \\
\hline Minimum & .10 & 6.2 & .05 & .23 & 11 & e.25 & .21 \\
\hline Median & .35 & 8.9 & .11 & .50 & 21 & e.66 & .43 \\
\hline $\mathrm{Mgal} / \mathrm{d} / \mathbf{m i}^{2}$ & .97 & - & .45 & 1.14 & - & e.76 & - \\
\hline Inches & 1.67 & - & .78 & 1.97 & - & e1.32 & - \\
\hline
\end{tabular}


Table 8. Daily, monthly, and annual statistics for discharge for U.S. Geological Survey stations in the drinking-water source area for Cambridge, Massachusetts, for water year 2005.-Continued

[Units are in millions of gallons. e, estimated; —, no data; $\mathrm{Mgal} / \mathrm{d} / \mathrm{mi}^{2}$, million gallons per day per square mile; \%, percent]

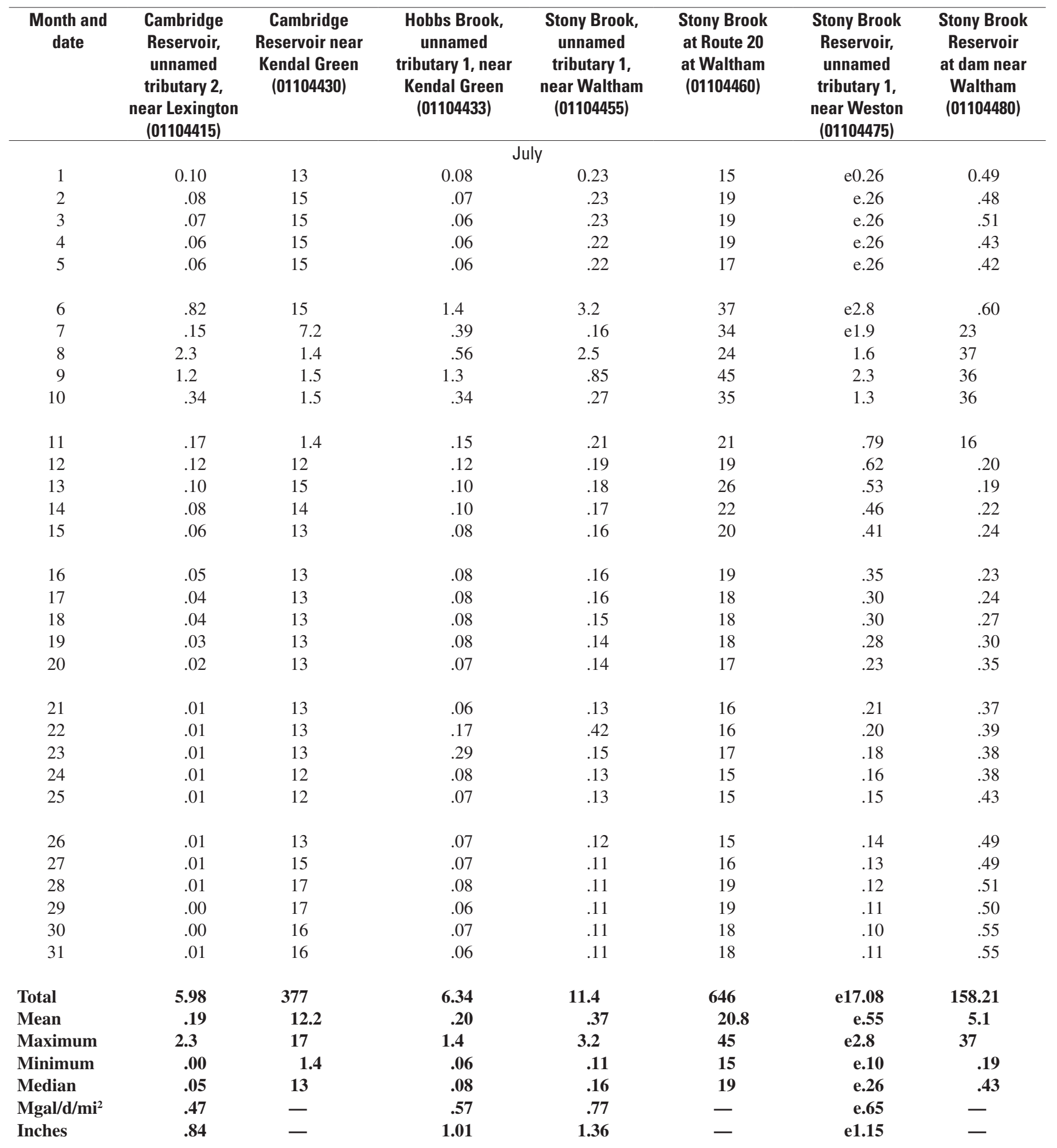


Table 8. Daily, monthly, and annual statistics for discharge for U.S. Geological Survey stations in the drinking-water source area for Cambridge, Massachusetts, for water year 2005.-Continued

[Units are in millions of gallons. e, estimated; -, no data; $\mathrm{Mgal} / \mathrm{d} / \mathrm{mi}^{2}$, million gallons per day per square mile; \%, percent]

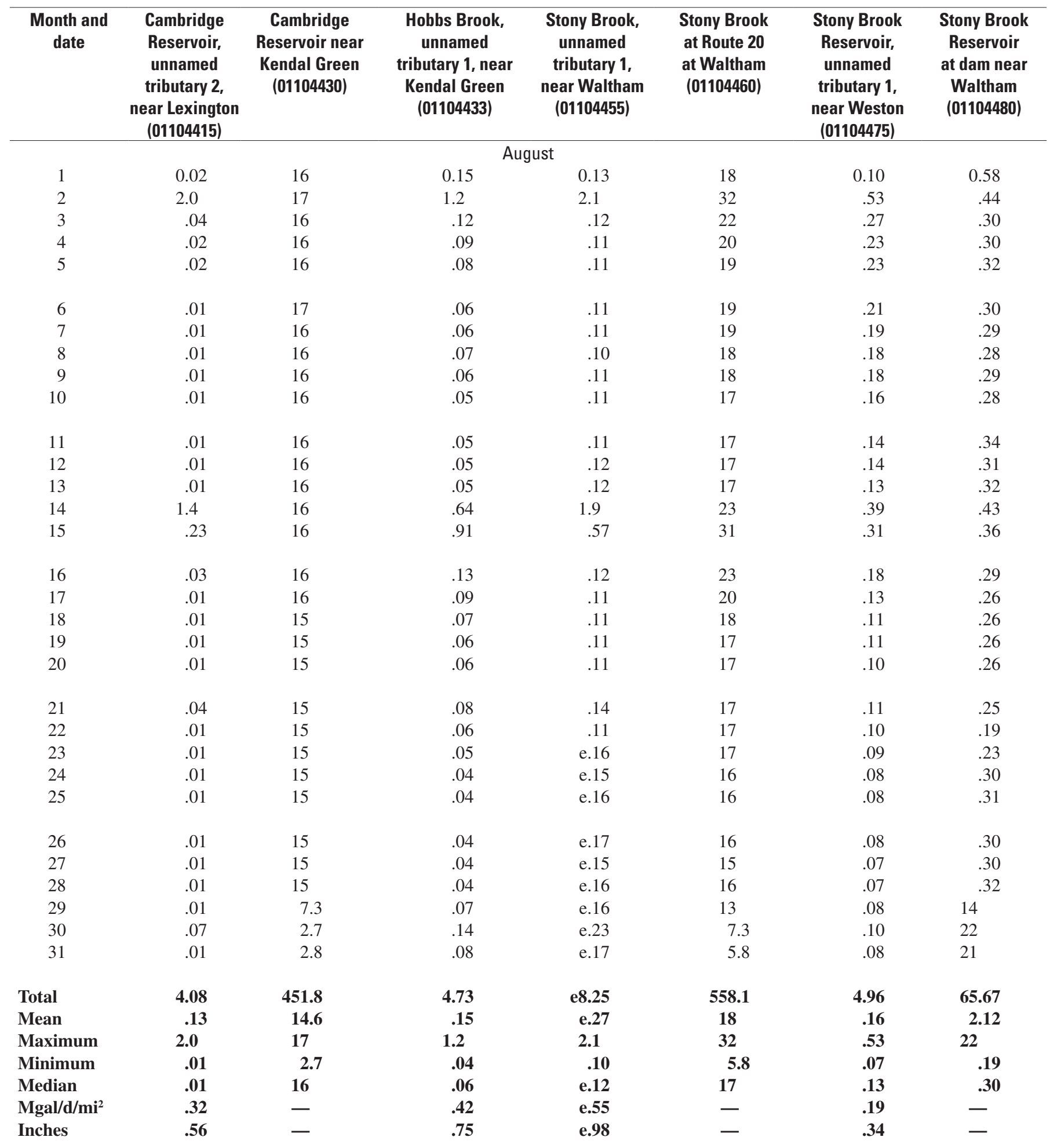


Table 8. Daily, monthly, and annual statistics for discharge for U.S. Geological Survey stations in the drinking-water source area for Cambridge, Massachusetts, for water year 2005.-Continued

[Units are in millions of gallons. e, estimated; —, no data; $\mathrm{Mgal} / \mathrm{d} / \mathrm{mi}^{2}$, million gallons per day per square mile; \%, percent]

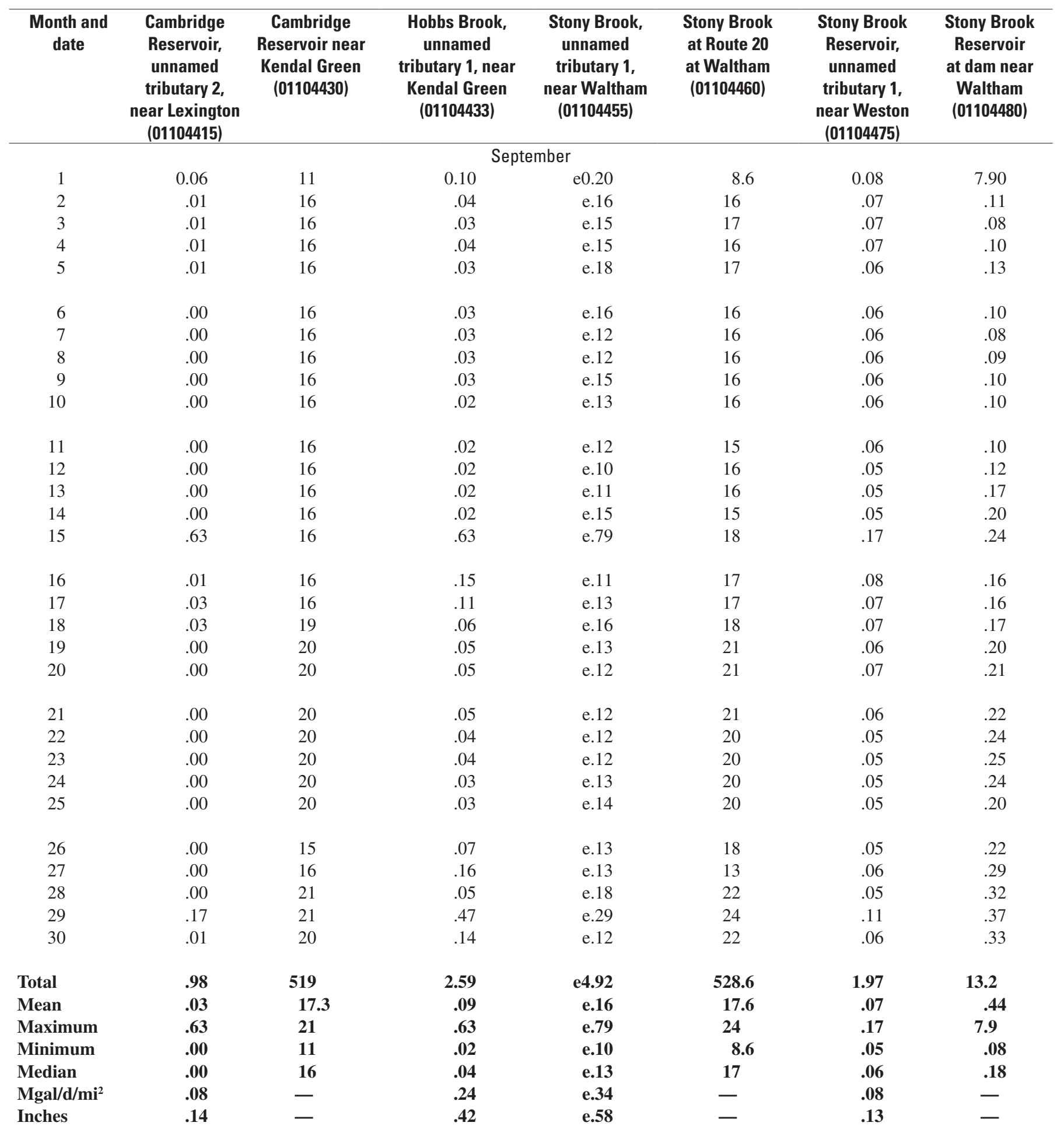


Table 8. Daily, monthly, and annual statistics for discharge for U.S. Geological Survey stations in the drinking-water source area for Cambridge, Massachusetts, for water year 2005.-Continued

[Units are in millions of gallons. e, estimated; —, no data; $\mathrm{Mgal} / \mathrm{d} / \mathrm{mi}^{2}$, million gallons per day per square mile; \%, percent]

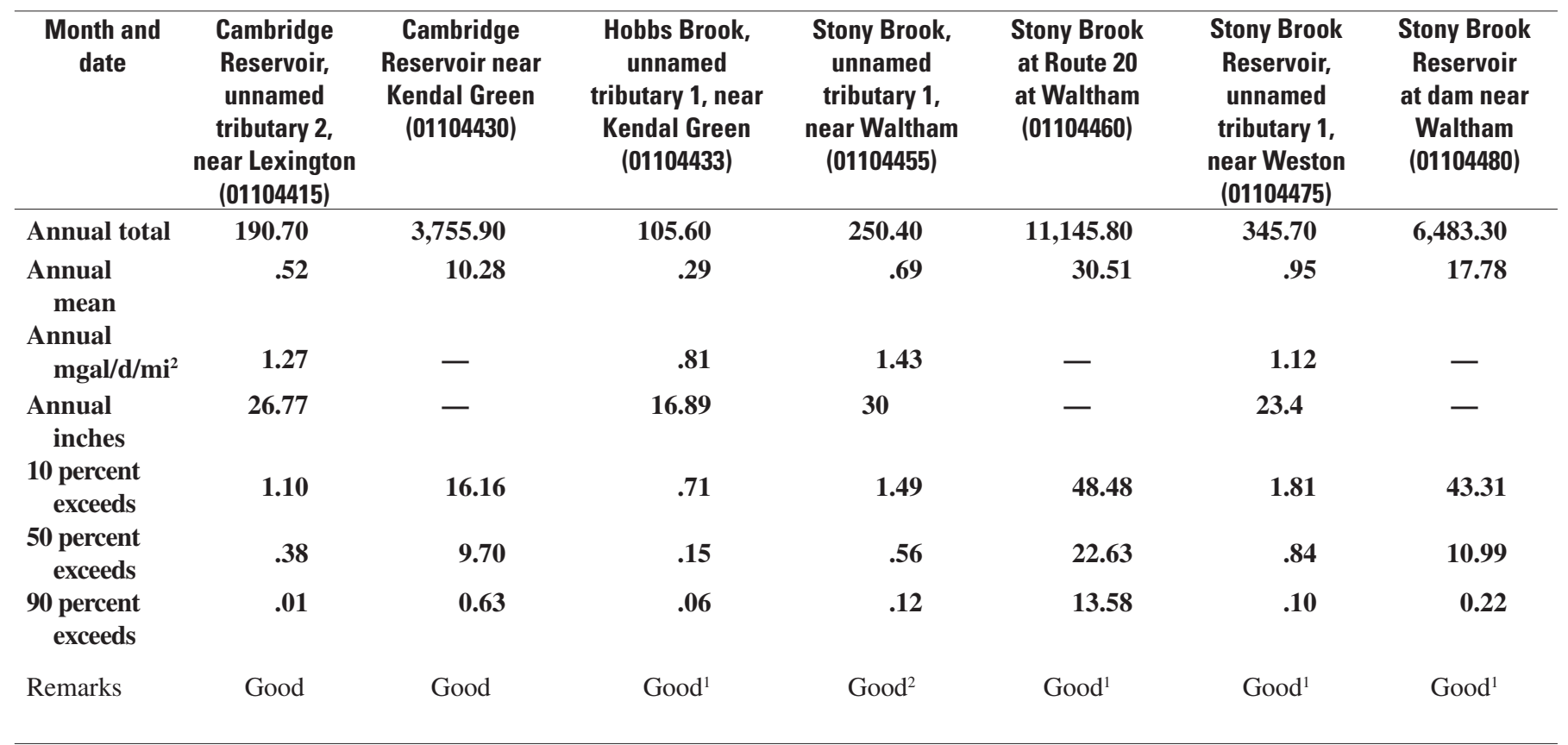

${ }^{1}$ Records that are estimated are rated as poor.

${ }^{2}$ Records that are estimated are rated as fair. 
Table 9. Daily, monthly, and annual statistics for reservoir altitude for U.S. Geological Survey station numbers 01104430, Cambridge Reservoir near Kendal Green, 01104480, Stony Brook Reservoir in Waltham, and 422302071083801, Fresh Pond Reservoir at Cambridge, Massachusetts, for water year 2005.

[Units are in feet relative to the City of Cambridge datum. Add 10.34 feet to altitude to adjust to National Geodetic Vertical Datum of 1929. e, estimated]

\begin{tabular}{cccc}
\hline & Cambridge & $\begin{array}{c}\text { Stony Brook } \\
\text { Reservoir }\end{array}$ & $\begin{array}{c}\text { Fresh Pond } \\
\text { gate house }\end{array}$ \\
$\begin{array}{c}\text { Month and } \\
\text { date }\end{array}$ & $\begin{array}{c}\text { Reservoir } \\
\text { near Kendal Green } \\
\text { (01104430) }\end{array}$ & $\begin{array}{c}\text { at dam near } \\
\text { Waltham } \\
\text { at Cambridge } \\
(01104480)\end{array}$ & $\begin{array}{c}\text { (422302071083801) } \\
\text { (010) }\end{array}$ \\
\end{tabular}

October

178.40

178.42

178.47

178.50

178.55

178.59

178.64

178.64

178.62

178.58

178.55

178.60

178.63

178.65

178.64

178.75

178.78

178.79

178.87

178.95

179.00

179.04

179.08

179.10

179.08

179.06

179.06

179.05

179.04

179.04

179.01

Mean

Maximum

178.78

179.10

178.40
78.50

77.99

77.50

77.49

77.56

77.49

77.35

77.57

77.81

77.94

77.96

77.75

77.40

77.05

76.84

77.19

77.53

77.65

77.66

77.74

77.68

77.52

77.30

77.05

76.86

76.69

76.52

76.33

76.15

76.04

76.11

77.30

78.50

76.04
16.68

16.68

16.68

16.66

16.63

16.59

16.53

16.3

16.12

16.09

16.09

16.09

16.05

16.03

16.06

16.18

16.22

16.23

16.31

16.35

16.4

16.42

16.44

16.52

16.52

16.53

16.56

16.59

16.61

16.62

16.62

16.4

16.68

16.03

\begin{tabular}{|c|c|c|c|}
\hline $\begin{array}{l}\text { Month and } \\
\text { date }\end{array}$ & $\begin{array}{c}\text { Cambridge } \\
\text { Reservoir } \\
\text { near Kendal Green } \\
(01104430)\end{array}$ & $\begin{array}{l}\text { Stony Brook } \\
\text { Reservoir } \\
\text { at dam near } \\
\text { Waltham } \\
\text { (01104480) }\end{array}$ & $\begin{array}{c}\text { Fresh Pond } \\
\text { gate house } \\
\text { at Cambridge } \\
(422302071083801)\end{array}$ \\
\hline
\end{tabular}

November

$\begin{array}{lll}178.94 & 76.24 & 16.59\end{array}$

$\begin{array}{lll}178.90 & 76.31 & 16.57\end{array}$

$\begin{array}{lll}178.86 & 76.40 & 16.58\end{array}$

$\begin{array}{lll}178.83 & 76.46 & 16.58\end{array}$

$\begin{array}{lll}178.88 & 76.88 & 16.59\end{array}$

$\begin{array}{lll}178.86 & 77.21 & 16.58\end{array}$

$\begin{array}{lll}178.84 & 77.44 & 16.59\end{array}$

$\begin{array}{lll}178.81 & 77.63 & 16.59\end{array}$

$\begin{array}{lll}178.78 & 77.74 & 16.58\end{array}$

$\begin{array}{lll}178.75 & 77.81 & 16.59\end{array}$

$\begin{array}{lll}178.70 & 77.87 & 16.58\end{array}$

$\begin{array}{lll}178.69 & 77.94 & 16.58\end{array}$

$\begin{array}{lll}178.73 & 78.09 & 16.64\end{array}$

$\begin{array}{lll}178.70 & 78.21 & 16.66\end{array}$

$\begin{array}{lll}178.67 & 78.31 & 16.68\end{array}$

$\begin{array}{lll}178.63 & 78.44 & 16.72\end{array}$

$\begin{array}{lll}178.61 & 78.58 & 16.74\end{array}$

$\begin{array}{lll}178.59 & 78.79 & 16.71\end{array}$

$\begin{array}{lll}178.58 & 78.93 & 16.66\end{array}$

$\begin{array}{lll}178.58 & 78.96 & 16.61\end{array}$

$\begin{array}{lll}178.59 & 78.97 & 16.58\end{array}$

$\begin{array}{lll}178.59 & 78.98 & 16.55\end{array}$

$\begin{array}{lll}178.59 & 78.91 & 16.51\end{array}$

$\begin{array}{lll}178.60 & 78.77 & 16.48\end{array}$

$\begin{array}{lll}178.67 & 78.82 & 16.48\end{array}$

$\begin{array}{lll}178.72 & 78.98 & 16.48\end{array}$

$\begin{array}{lll}178.73 & 78.99 & 16.55\end{array}$

$\begin{array}{lll}178.79 & 79.04 & 16.55\end{array}$

$\begin{array}{lll}178.95 & 79.88 & 16.63\end{array}$

$\begin{array}{lll}179.01 & 80.29 & 16.62\end{array}$

$\begin{array}{llll}\text { Mean } & 178.74 & 78.20 & 16.59 \\ \text { Maximum } & 179.01 & 80.29 & 16.74 \\ \text { Minimum } & 178.58 & 76.24 & 16.48\end{array}$


Table 9. Daily, monthly, and annual statistics for reservoir altitude for U.S. Geological Survey station numbers 01104430 , Cambridge Reservoir near Kendal Green, 01104480, Stony Brook Reservoir in Waltham, and 422302071083801, Fresh Pond Reservoir at Cambridge, Massachusetts, for water year 2005. - Continued

[Units are in feet relative to the City of Cambridge datum. Add 10.34 feet to altitude to adjust to National Geodetic Vertical Datum of 1929. e, estimated]

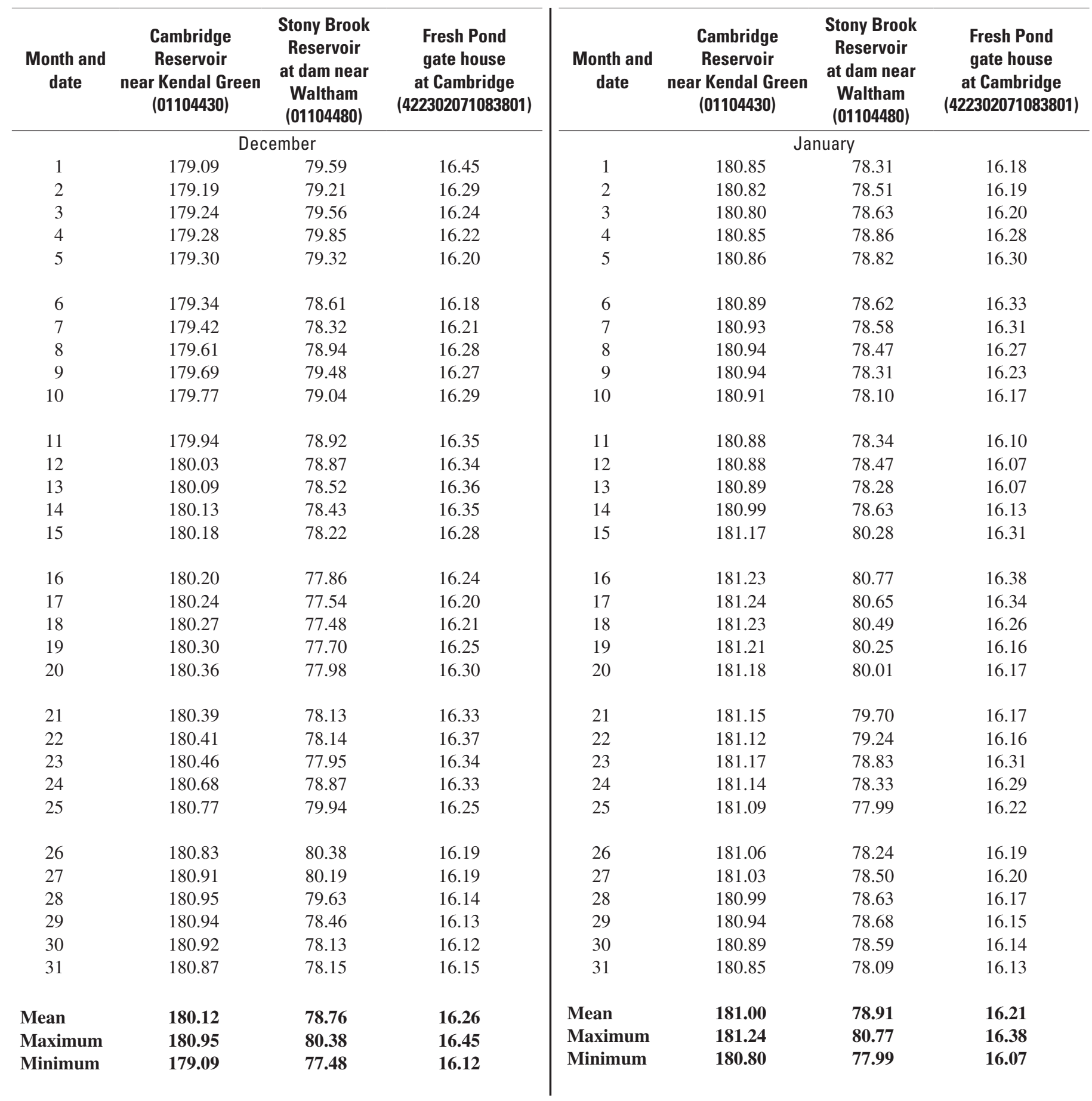


Table 9. Daily, monthly, and annual statistics for reservoir altitude for U.S. Geological Survey station numbers 01104430 , Cambridge Reservoir near Kendal Green, 01104480, Stony Brook Reservoir in Waltham, and 422302071083801, Fresh Pond Reservoir at Cambridge, Massachusetts, for water year 2005.-Continued

[Units are in feet relative to the City of Cambridge datum. Add 10.34 feet to altitude to adjust to National Geodetic Vertical Datum of 1929. e, estimated]

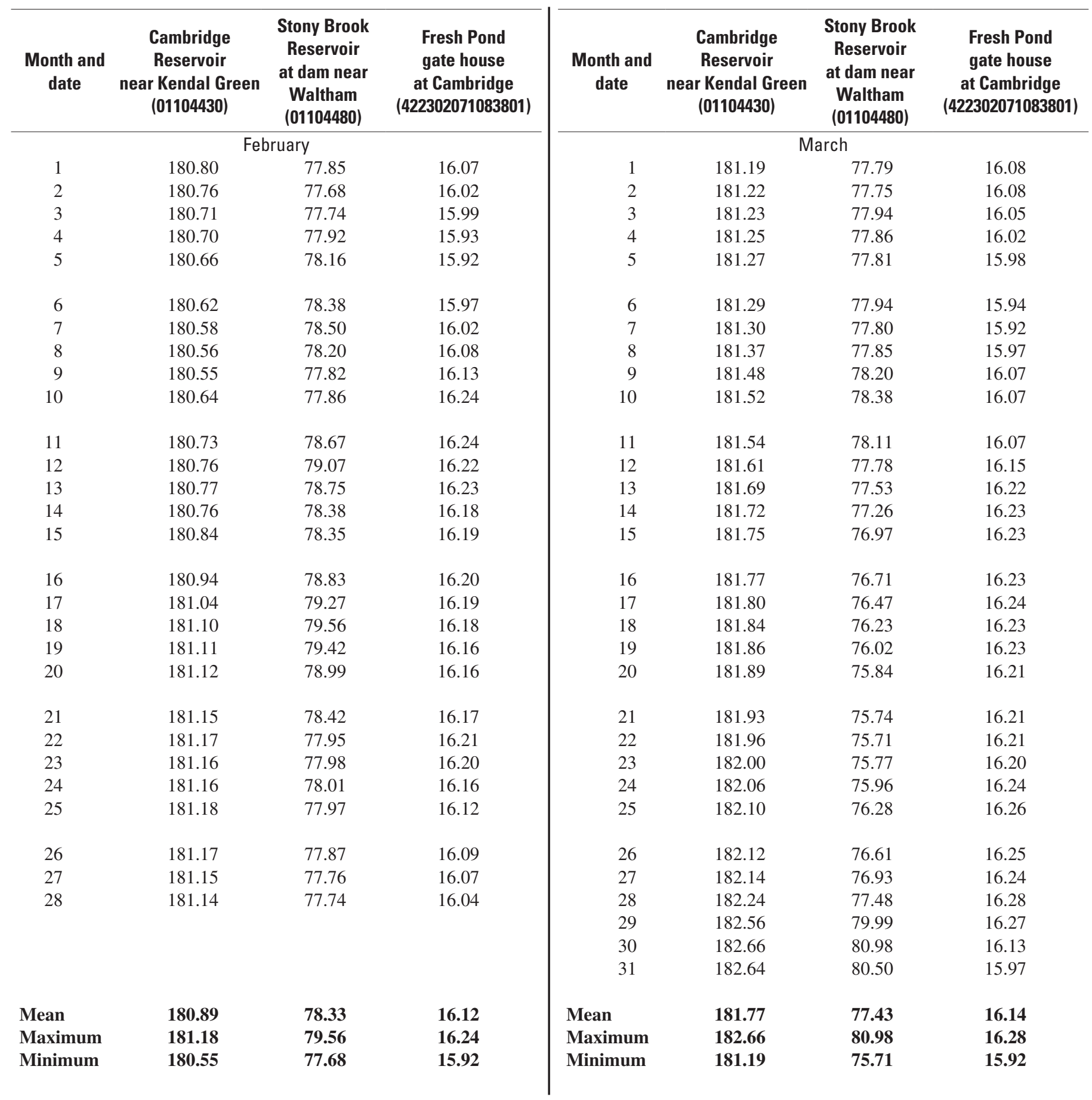


Table 9. Daily, monthly, and annual statistics for reservoir altitude for U.S. Geological Survey station numbers 01104430 , Cambridge Reservoir near Kendal Green, 01104480, Stony Brook Reservoir in Waltham, and 422302071083801, Fresh Pond Reservoir at Cambridge, Massachusetts, for water year 2005. - Continued

[Units are in feet relative to the City of Cambridge datum. Add 10.34 feet to altitude to adjust to National Geodetic Vertical Datum of 1929. e, estimated]

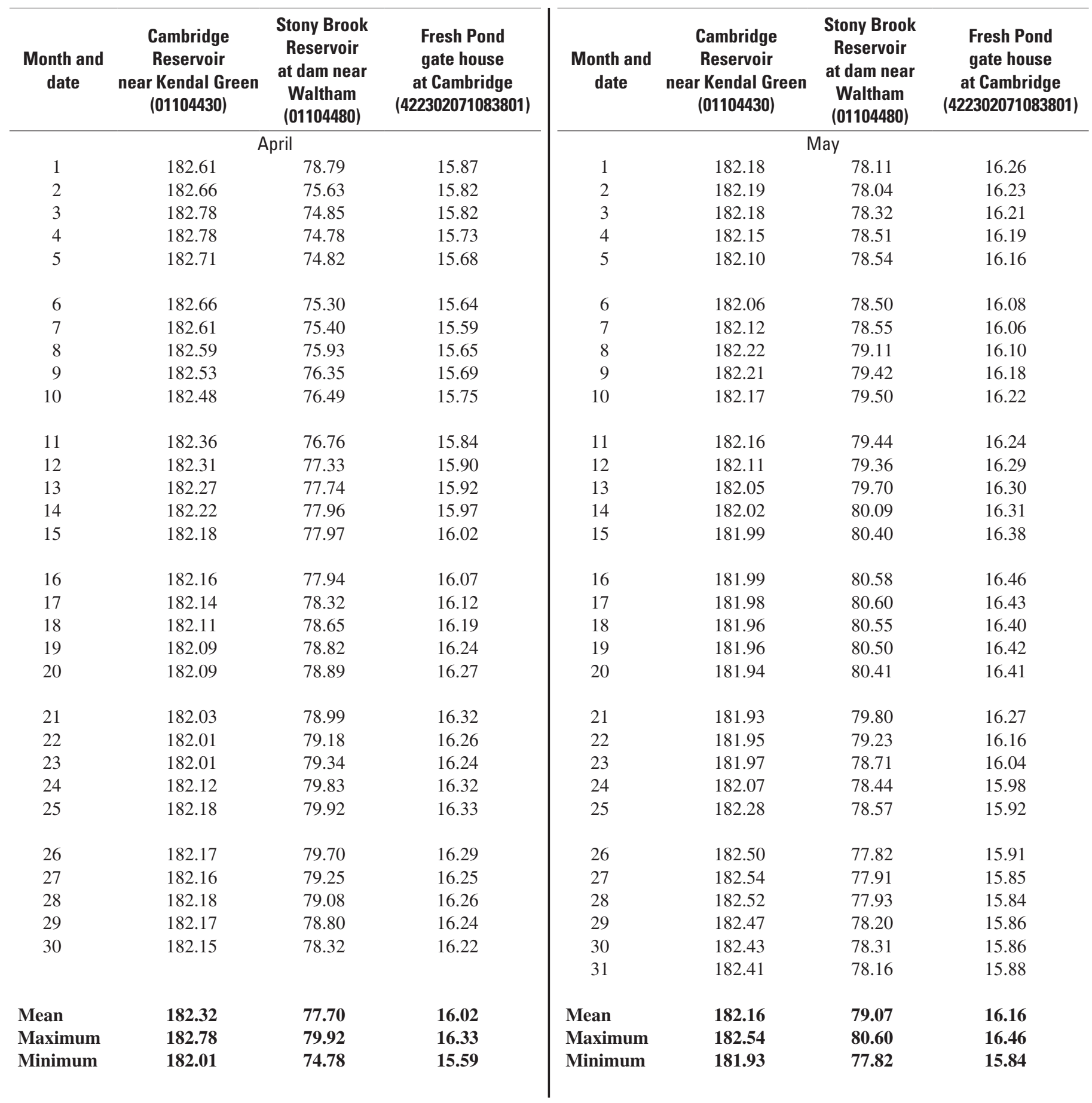


Table 9. Daily, monthly, and annual statistics for reservoir altitude for U.S. Geological Survey station numbers 01104430 , Cambridge Reservoir near Kendal Green, 01104480, Stony Brook Reservoir in Waltham, and 422302071083801, Fresh Pond Reservoir at Cambridge, Massachusetts, for water year 2005.-Continued

[Units are in feet relative to the City of Cambridge datum. Add 10.34 feet to altitude to adjust to National Geodetic Vertical Datum of 1929. e, estimated]

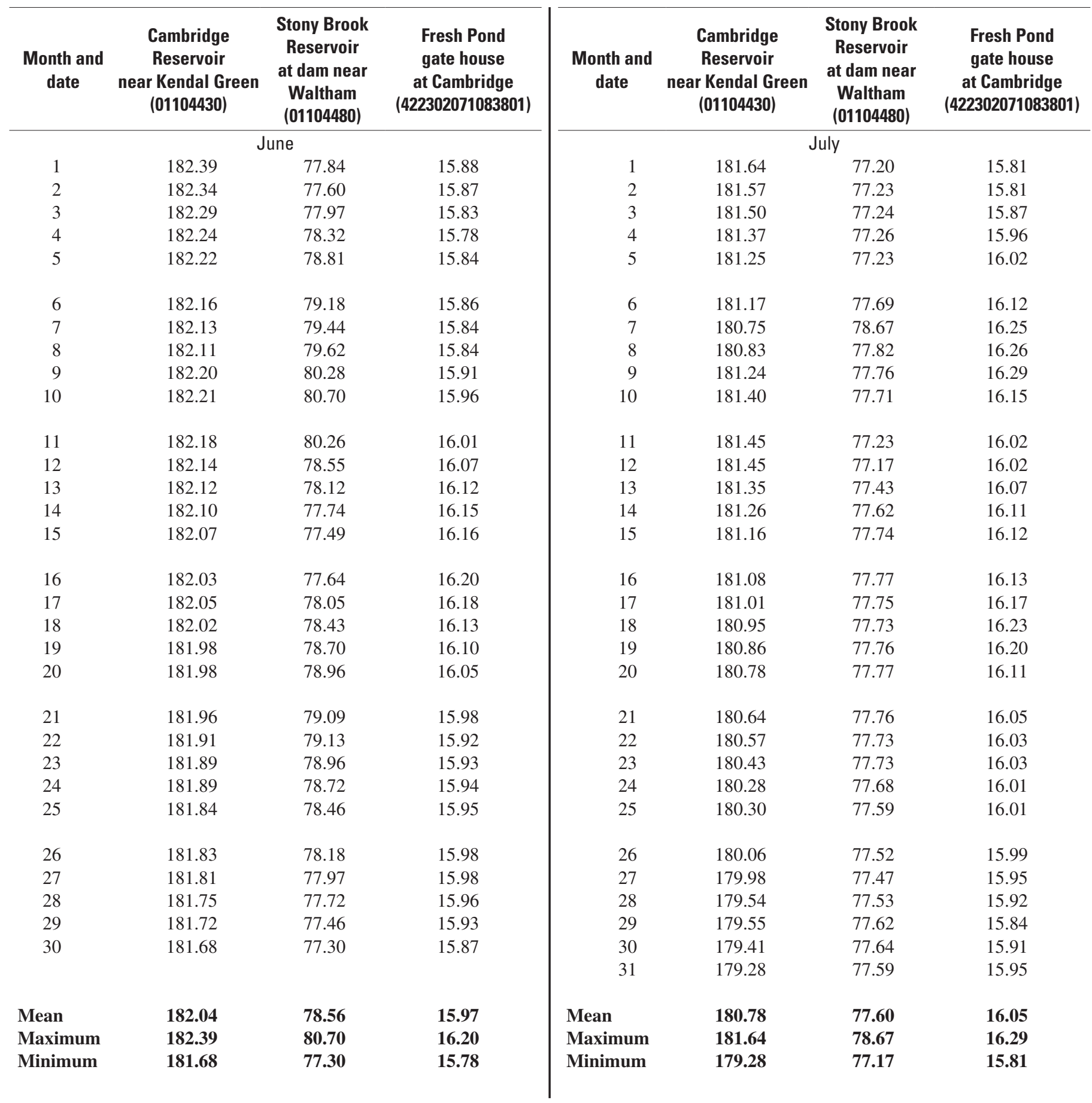


Table 9. Daily, monthly, and annual statistics for reservoir altitude for U.S. Geological Survey station numbers 01104430 , Cambridge Reservoir near Kendal Green, 01104480, Stony Brook Reservoir in Waltham, and 422302071083801, Fresh Pond Reservoir at Cambridge, Massachusetts, for water year 2005. - Continued

[Units are in feet relative to the City of Cambridge datum. Add 10.34 feet to altitude to adjust to National Geodetic Vertical Datum of 1929. e, estimated]

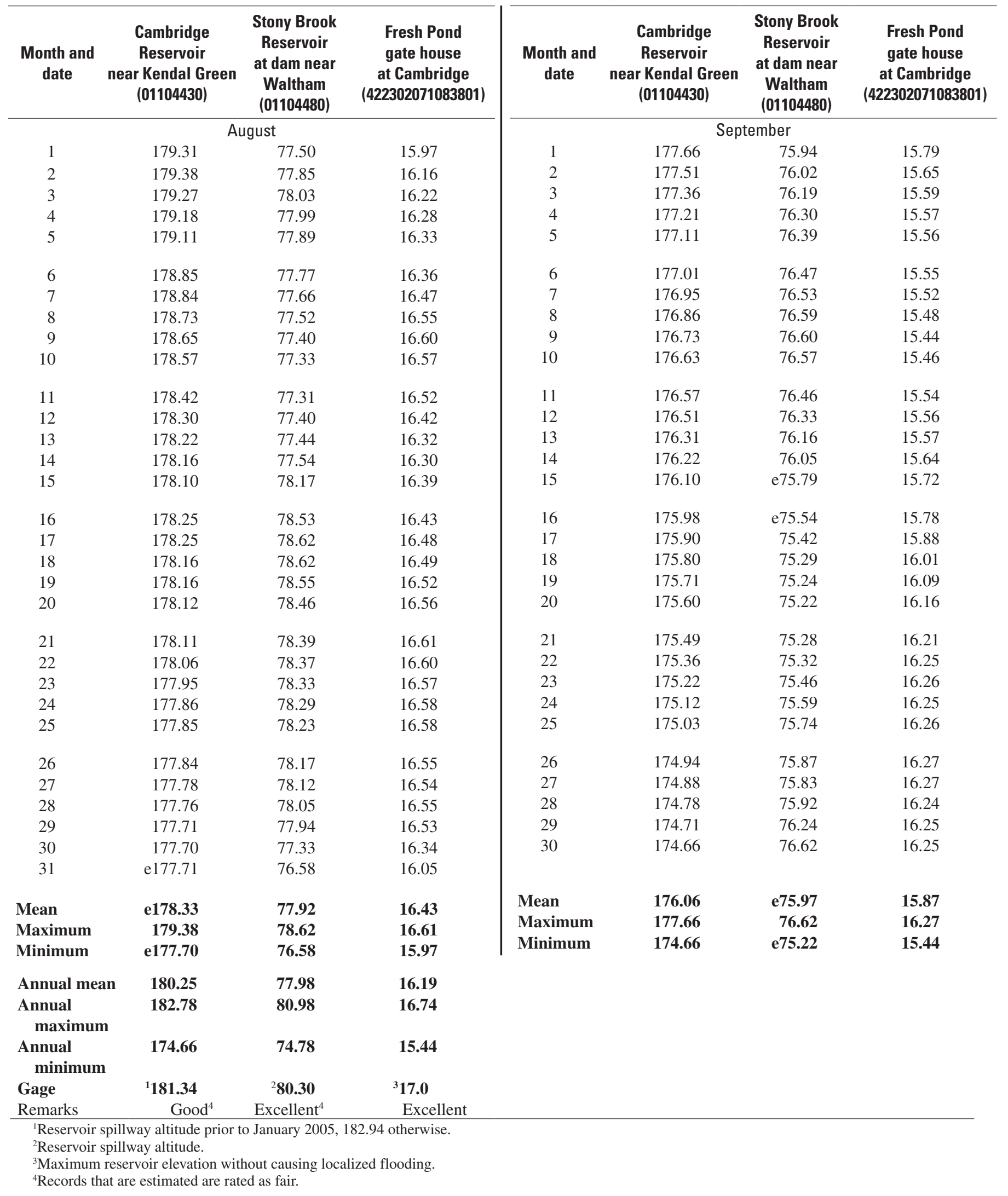


Table 10. Daily, monthly, and annual statistics for reservoir capacity for U.S. Geological Survey stations 01104430, Hobbs Brook below Cambridge Reservoir near Kendal Green; 01104480, Stony Brook Reservoir in Waltham; and 422302071083801, Fresh Pond Reservoir at Cambridge, Massachusetts, for water year 2005.

[Units are in millions of gallons. e, estimated]

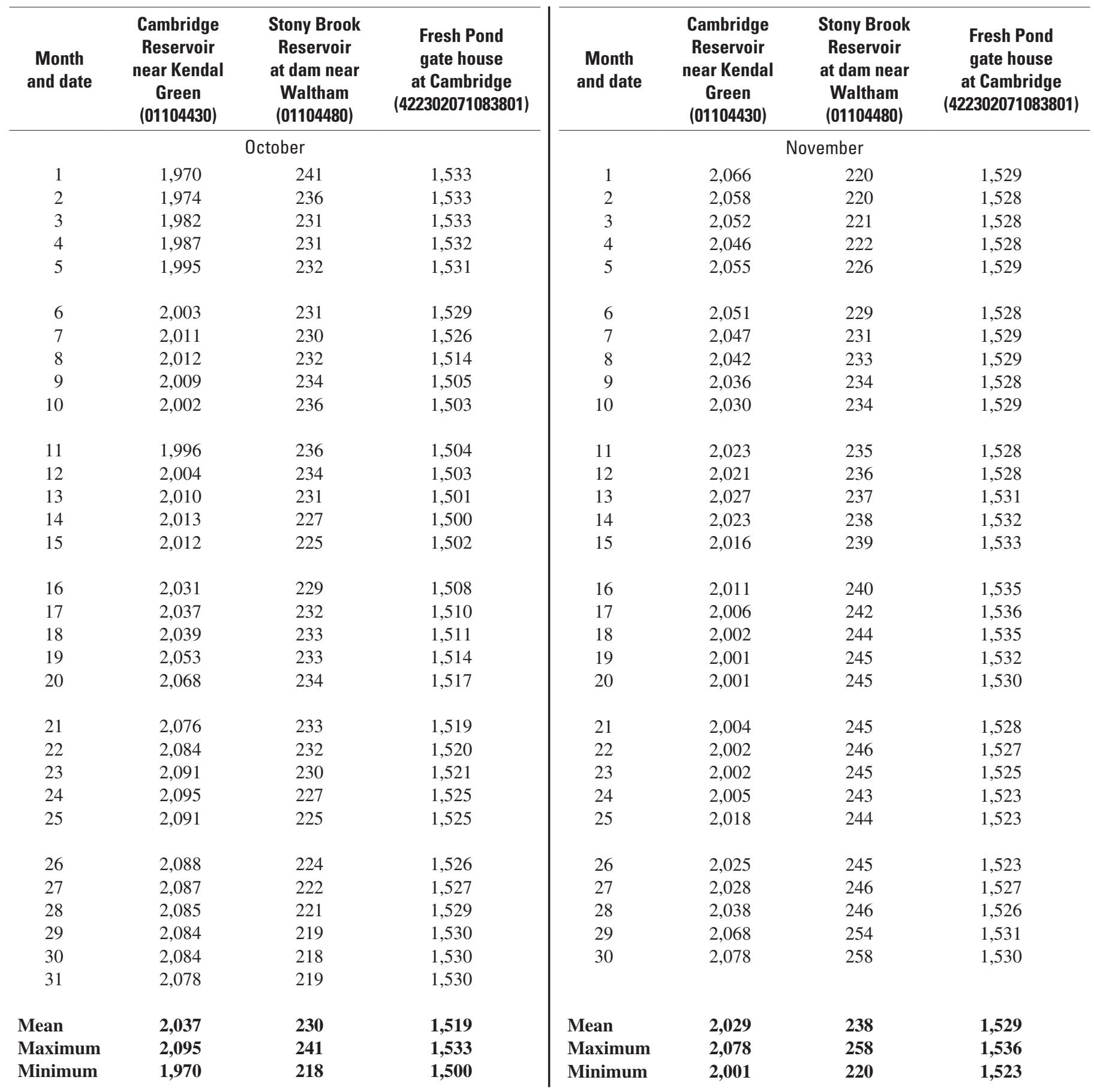


Table 10. Daily, monthly, and annual statistics for reservoir capacity for U.S. Geological Survey stations 01104430 , Hobbs Brook below Cambridge Reservoir near Kendal Green; 01104480, Stony Brook Reservoir in Waltham; and 422302071083801, Fresh Pond Reservoir at Cambridge, Massachusetts, for water year 2005. - Continued

[Units are in millions of gallons. e, estimated]

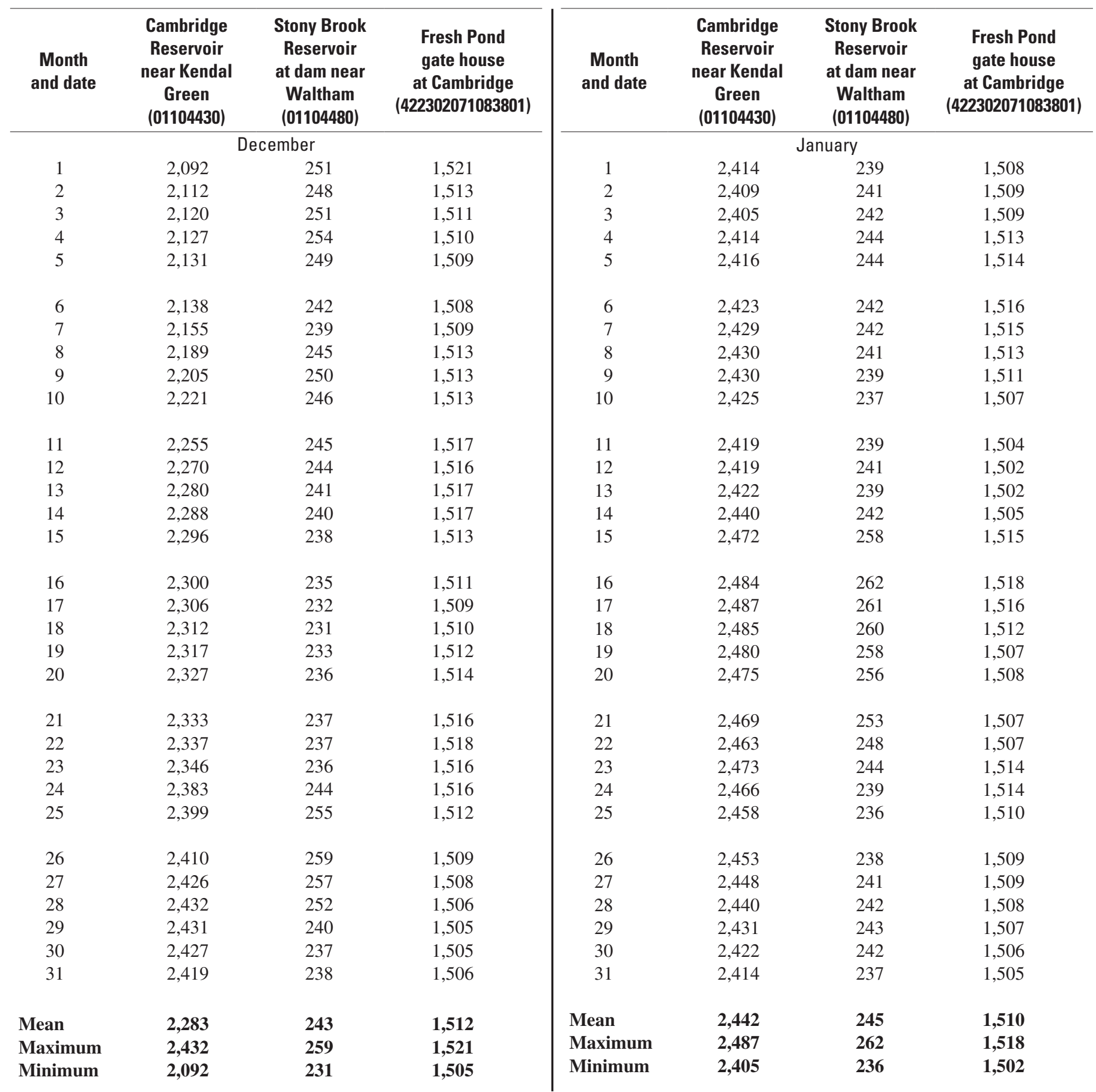


Table 10. Daily, monthly, and annual statistics for reservoir capacity for U.S. Geological Survey stations 01104430, Hobbs Brook below Cambridge Reservoir near Kendal Green; 01104480, Stony Brook Reservoir in Waltham; and 422302071083801, Fresh Pond Reservoir at Cambridge, Massachusetts, for water year 2005.-Continued

[Units are in millions of gallons. e, estimated]

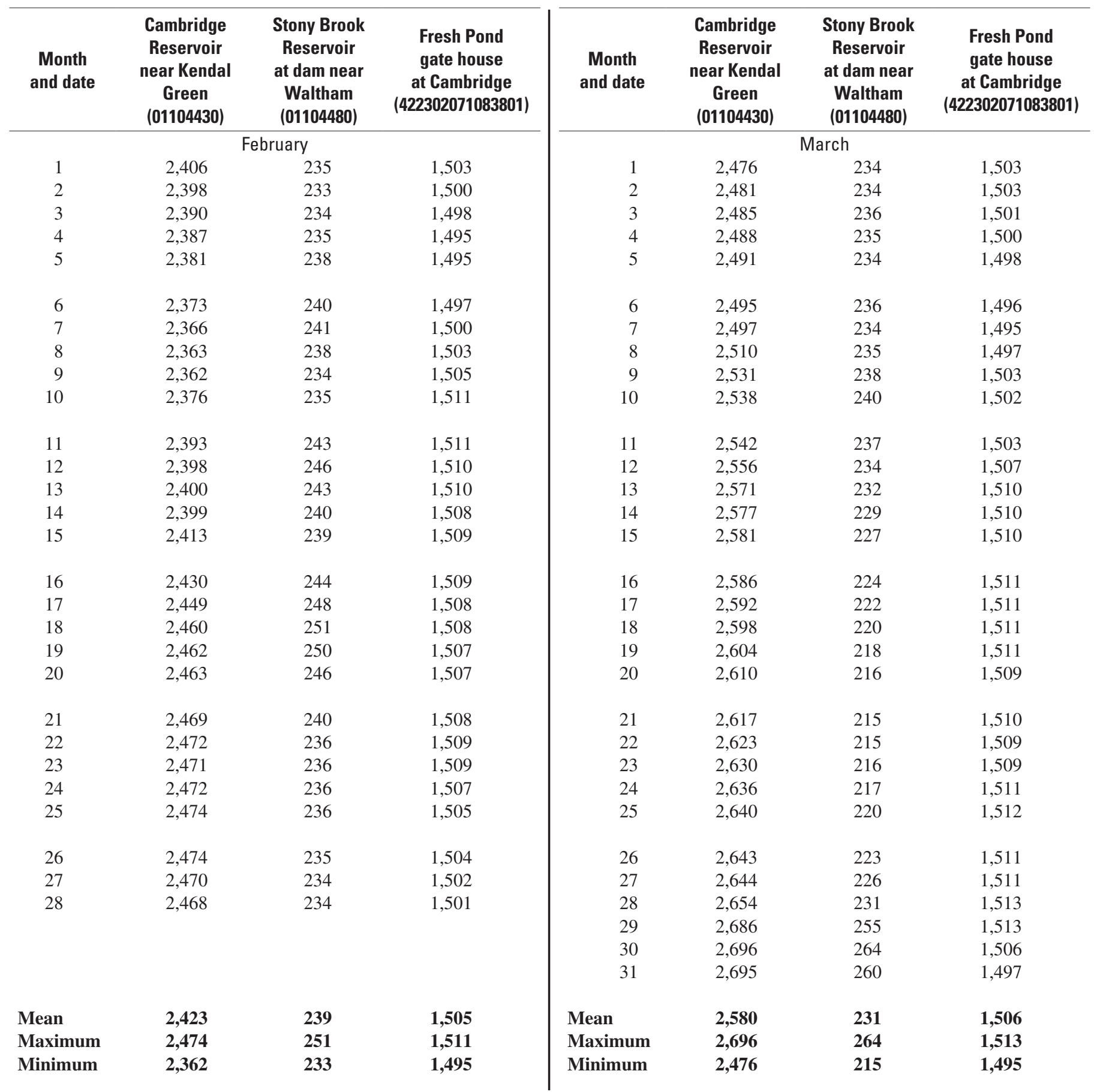


Table 10. Daily, monthly, and annual statistics for reservoir capacity for U.S. Geological Survey stations 01104430 , Hobbs Brook below Cambridge Reservoir near Kendal Green; 01104480, Stony Brook Reservoir in Waltham; and 422302071083801, Fresh Pond Reservoir at Cambridge, Massachusetts, for water year 2005. - Continued

[Units are in millions of gallons. e, estimated]

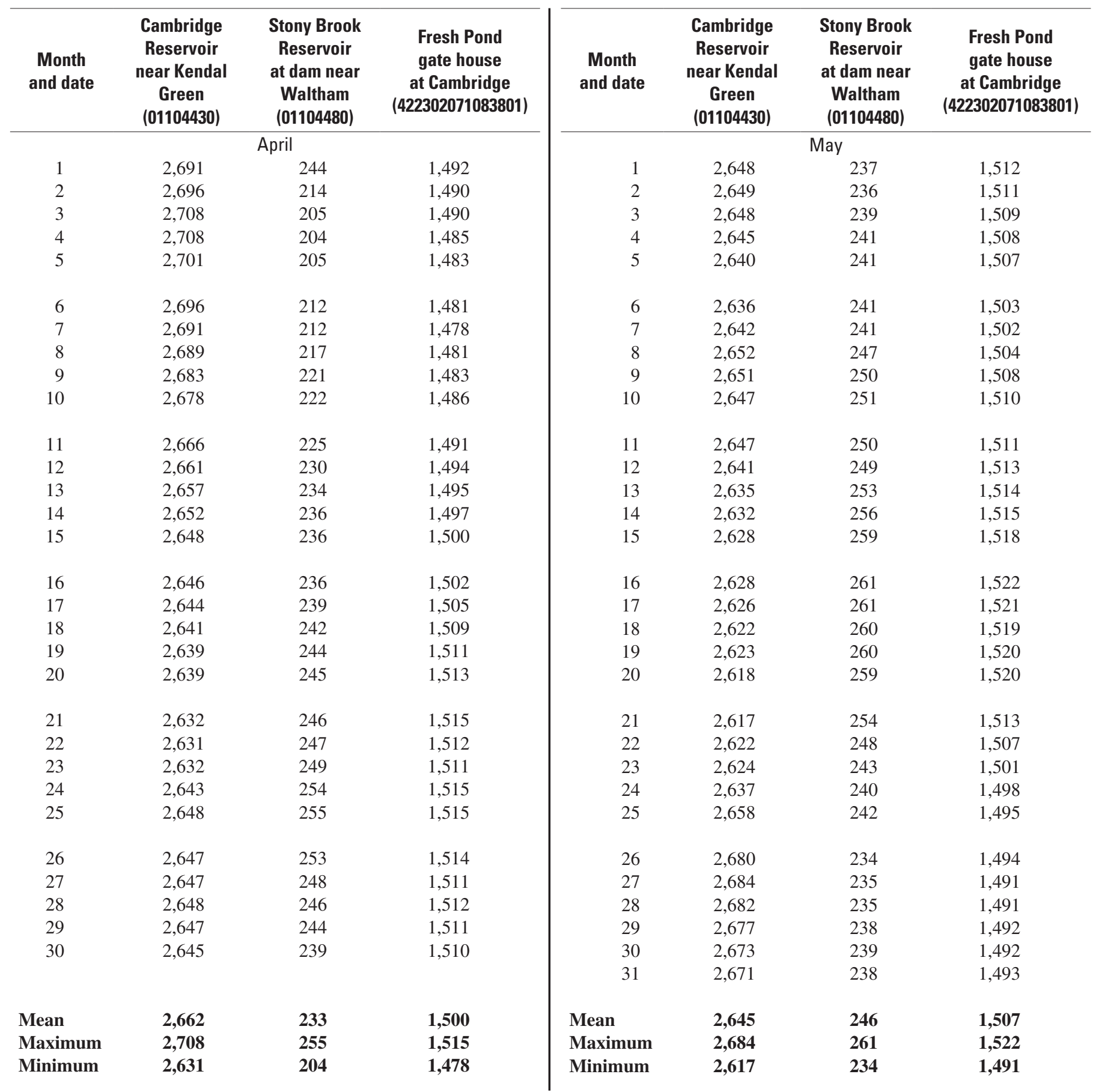


Table 10. Daily, monthly, and annual statistics for reservoir capacity for U.S. Geological Survey stations 01104430, Hobbs Brook below Cambridge Reservoir near Kendal Green; 01104480, Stony Brook Reservoir in Waltham; and 422302071083801, Fresh Pond Reservoir at Cambridge, Massachusetts, for water year 2005.-Continued

[Units are in millions of gallons. e, estimated]

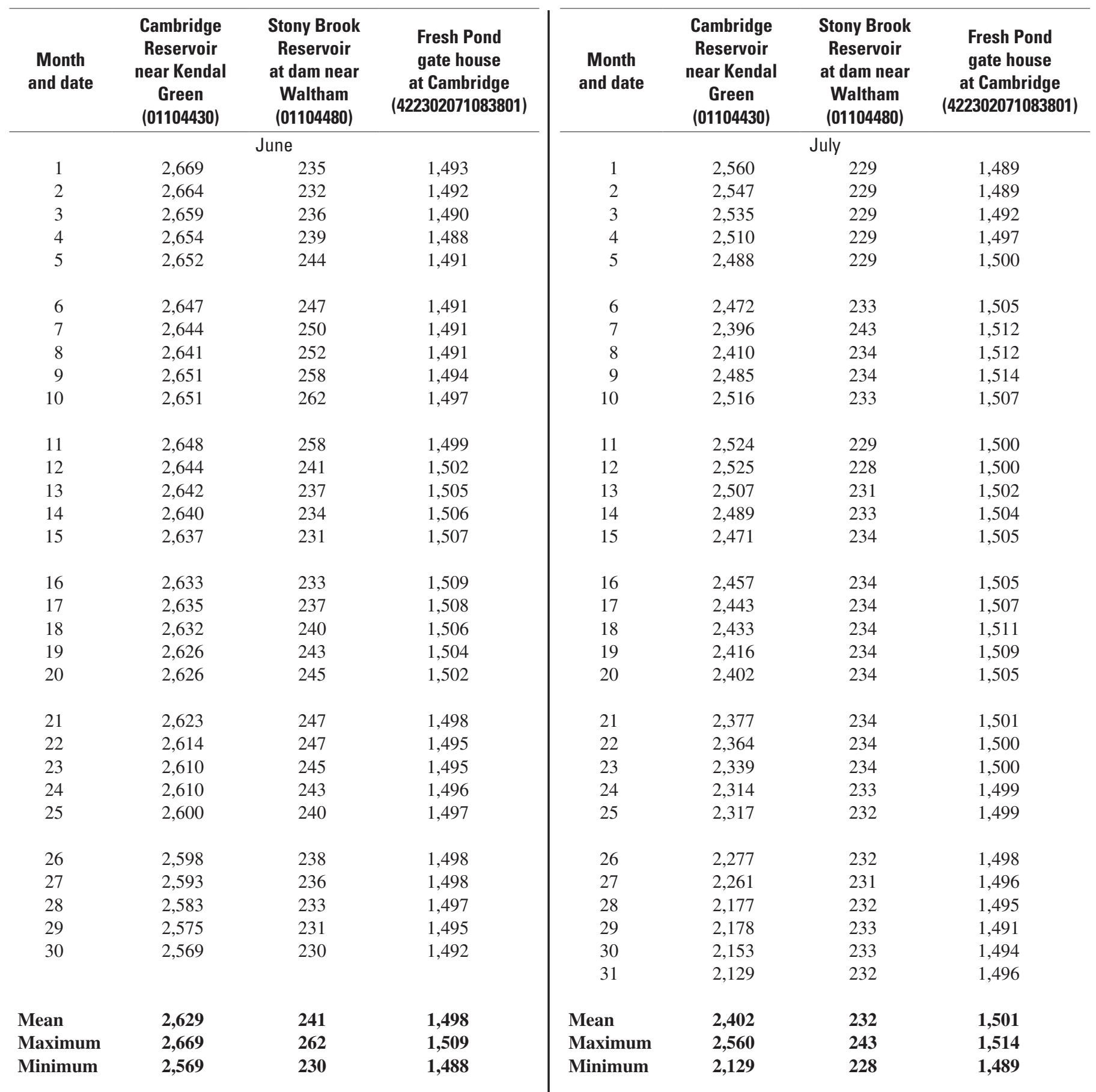


Table 10. Daily, monthly, and annual statistics for reservoir capacity for U.S. Geological Survey stations 01104430, Hobbs Brook below Cambridge Reservoir near Kendal Green; 01104480, Stony Brook Reservoir in Waltham; and 422302071083801, Fresh Pond Reservoir at Cambridge, Massachusetts, for water year 2005. - Continued

[Units are in millions of gallons. e, estimated]

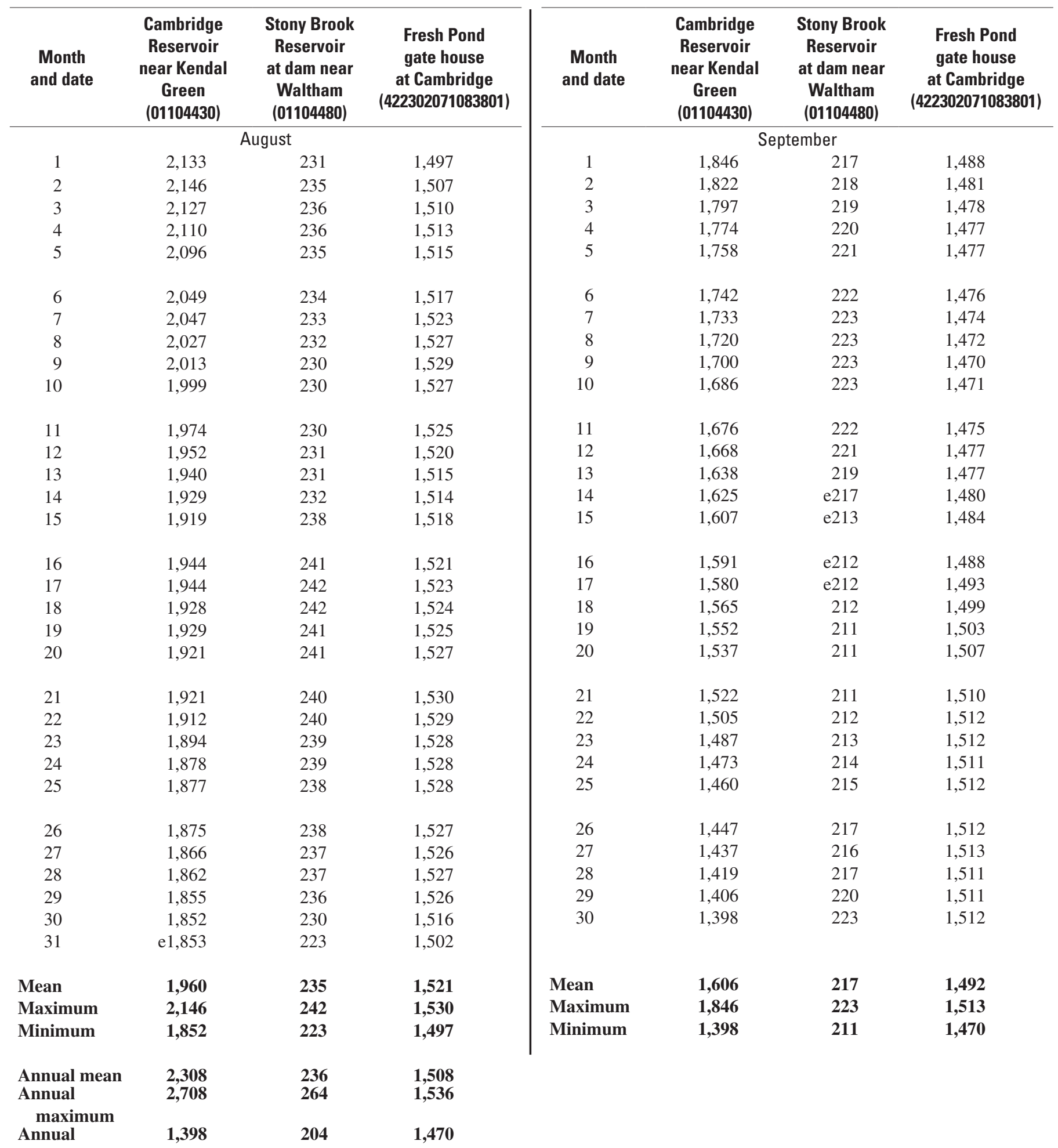
minimum

Remarks

${ }^{1}$ Reservoir storage capacity at spillway altitude is 2,503 million gallons prior to January 2005, otherwise storage capacity is 2,723 million gallons.

${ }^{2}$ Reservoir storage capacity at spillway altitude is 258 million gallons.

${ }^{3}$ Maximum reservoir storage capacity without causing localized flooding is 1,549 million gallons. 
Table 11. Daily, monthly, and annual statistics for precipitation for U.S. Geological Survey station numbers 01104430 , Cambridge Reservoir near Kendal Green, 01104480, Stony Brook Reservoir in Waltham, and 422302071083801, Fresh Pond Reservoir at Cambridge, water year 2005.

[Units are in inches. e, estimated]

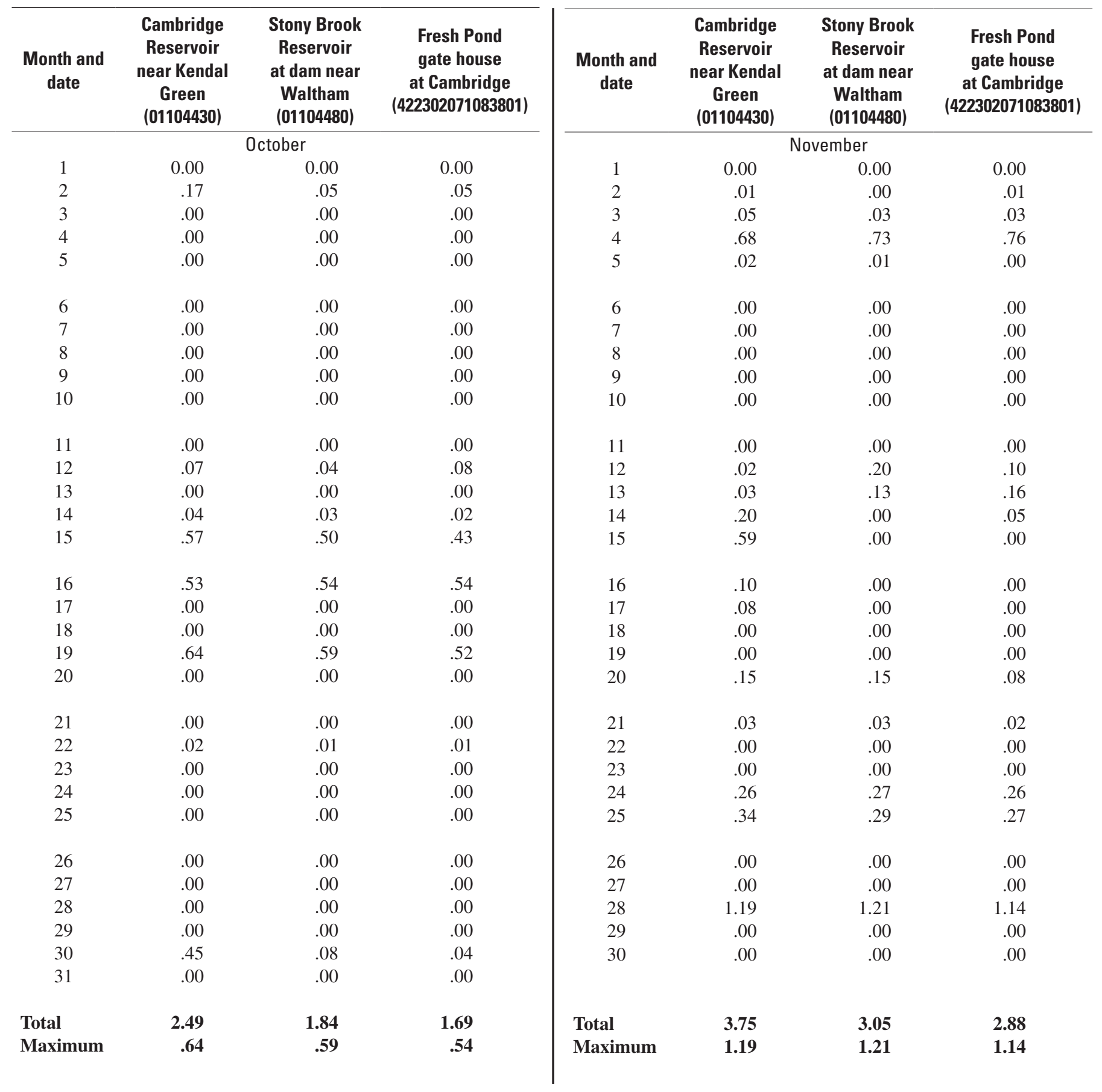


Table 11. Daily, monthly, and annual statistics for precipitation for U.S. Geological Survey stations 01104430 , Hobbs Brook below Cambridge Reservoir near Kendal Green; 01104480, Stony Brook Reservoir in Waltham; and 422302071083801, Fresh Pond Reservoir at Cambridge, Massachusetts, for water year 2005.—Continued

[Units are in inches; e, estimated]

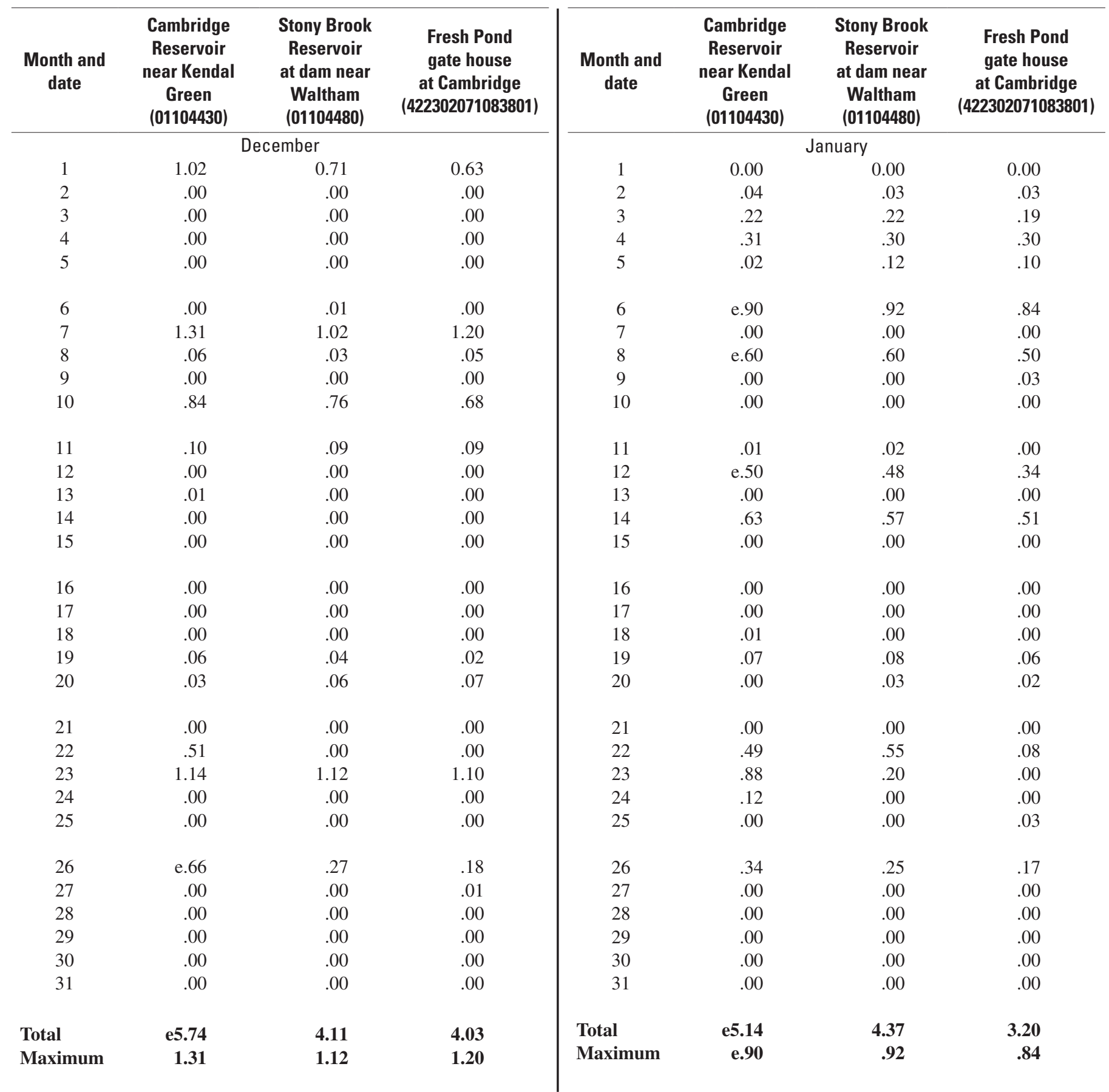


Table 11. Daily, monthly, and annual statistics for precipitation for U.S. Geological Survey stations 01104430 , Hobbs Brook below Cambridge Reservoir near Kendal Green; 01104480, Stony Brook Reservoir in Waltham; and 422302071083801, Fresh Pond Reservoir at Cambridge, Massachusetts, for water year 2005.-Continued

[Units are in inches; e, estimated]

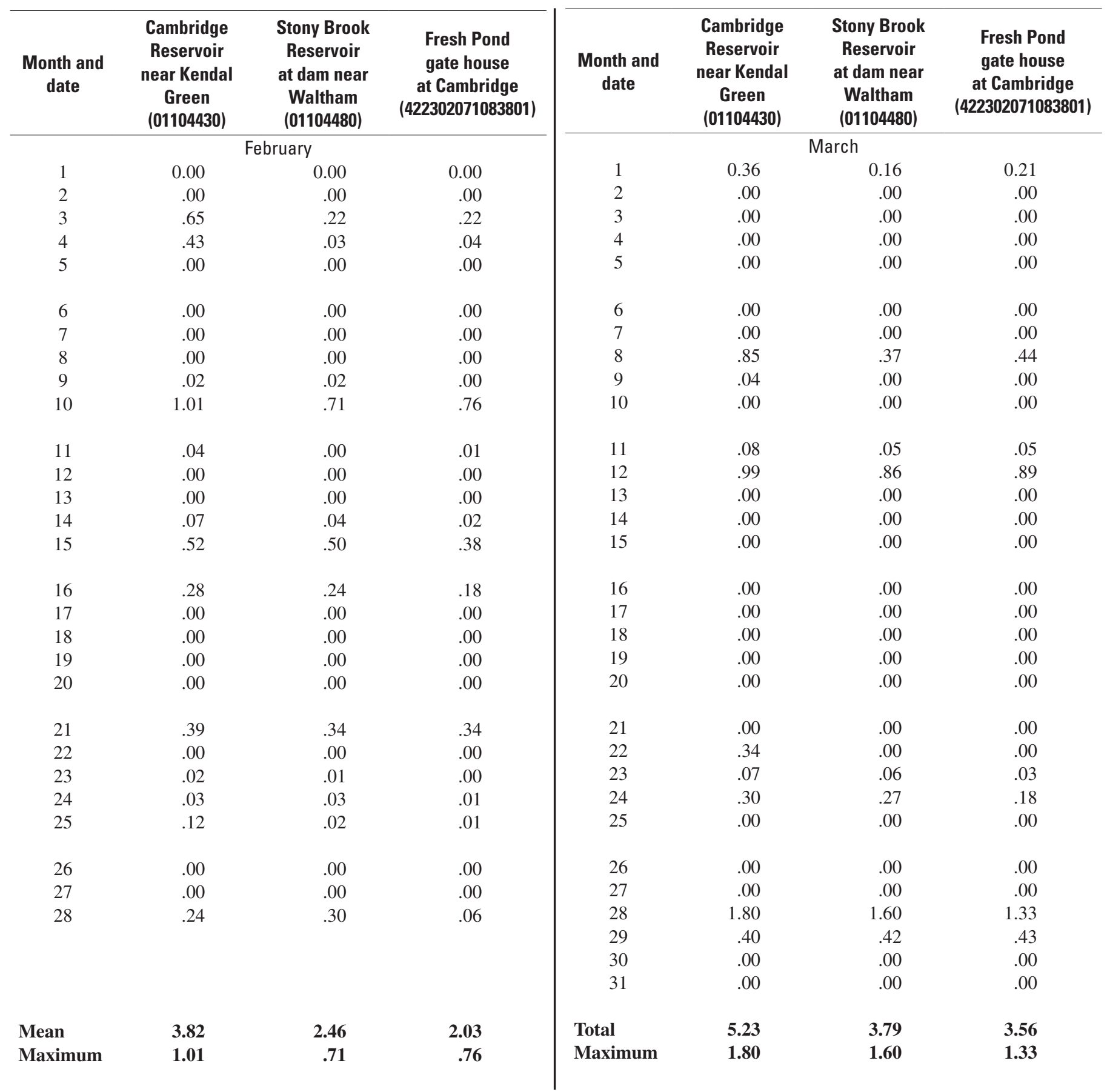


Table 11. Daily, monthly, and annual statistics for precipitation for U.S. Geological Survey stations 01104430 , Hobbs Brook below Cambridge Reservoir near Kendal Green; 01104480, Stony Brook Reservoir in Waltham; and 422302071083801, Fresh Pond Reservoir at Cambridge, Massachusetts, for water year 2005.—Continued

[Units are in inches; e, estimated]

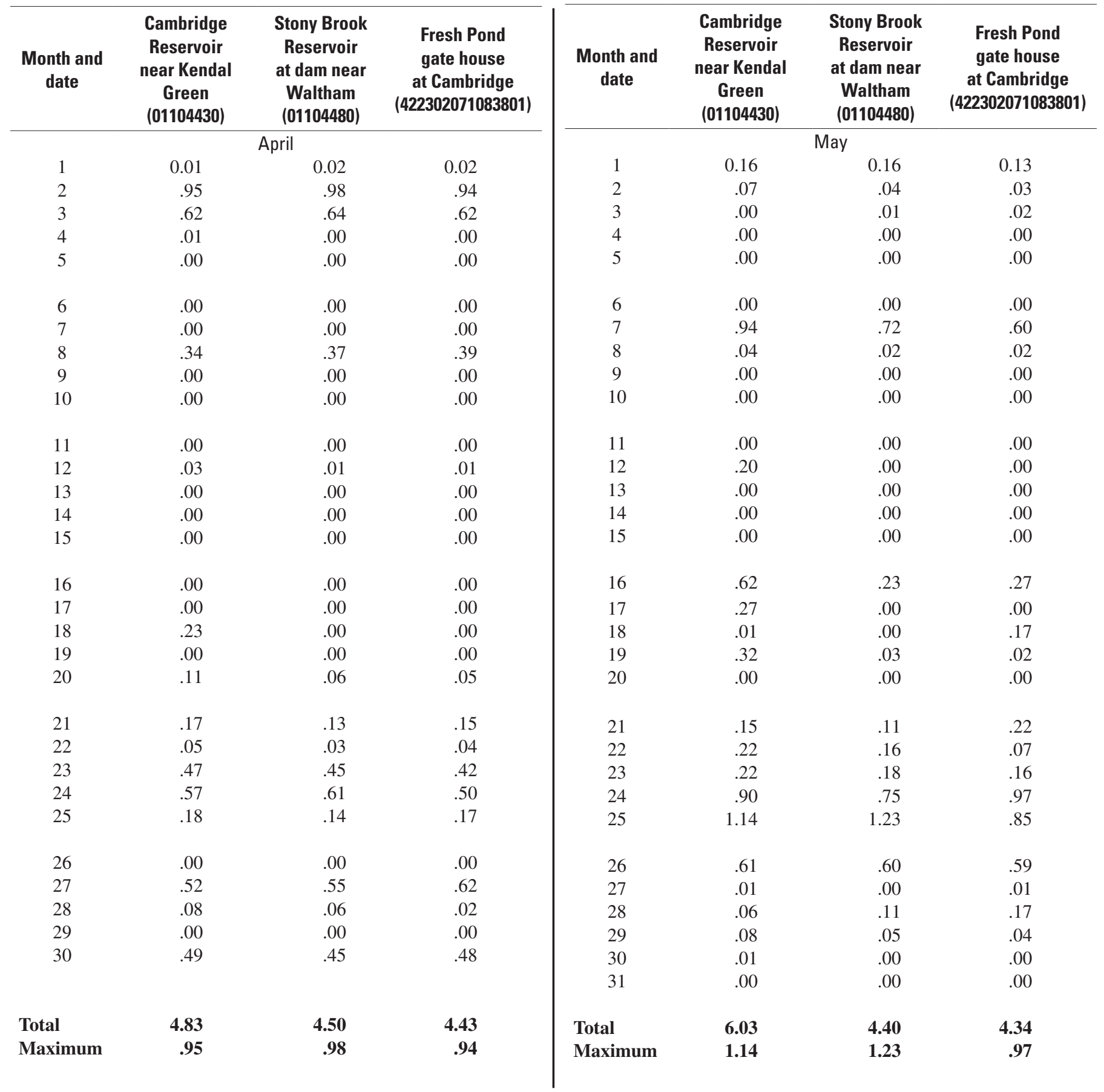


Table 11. Daily, monthly, and annual statistics for precipitation for U.S. Geological Survey stations 01104430 , Hobbs Brook below Cambridge Reservoir near Kendal Green; 01104480, Stony Brook Reservoir in Waltham; and 422302071083801, Fresh Pond Reservoir at Cambridge, Massachusetts, for water year 2005.—Continued

[Units are in inches; e, estimated]

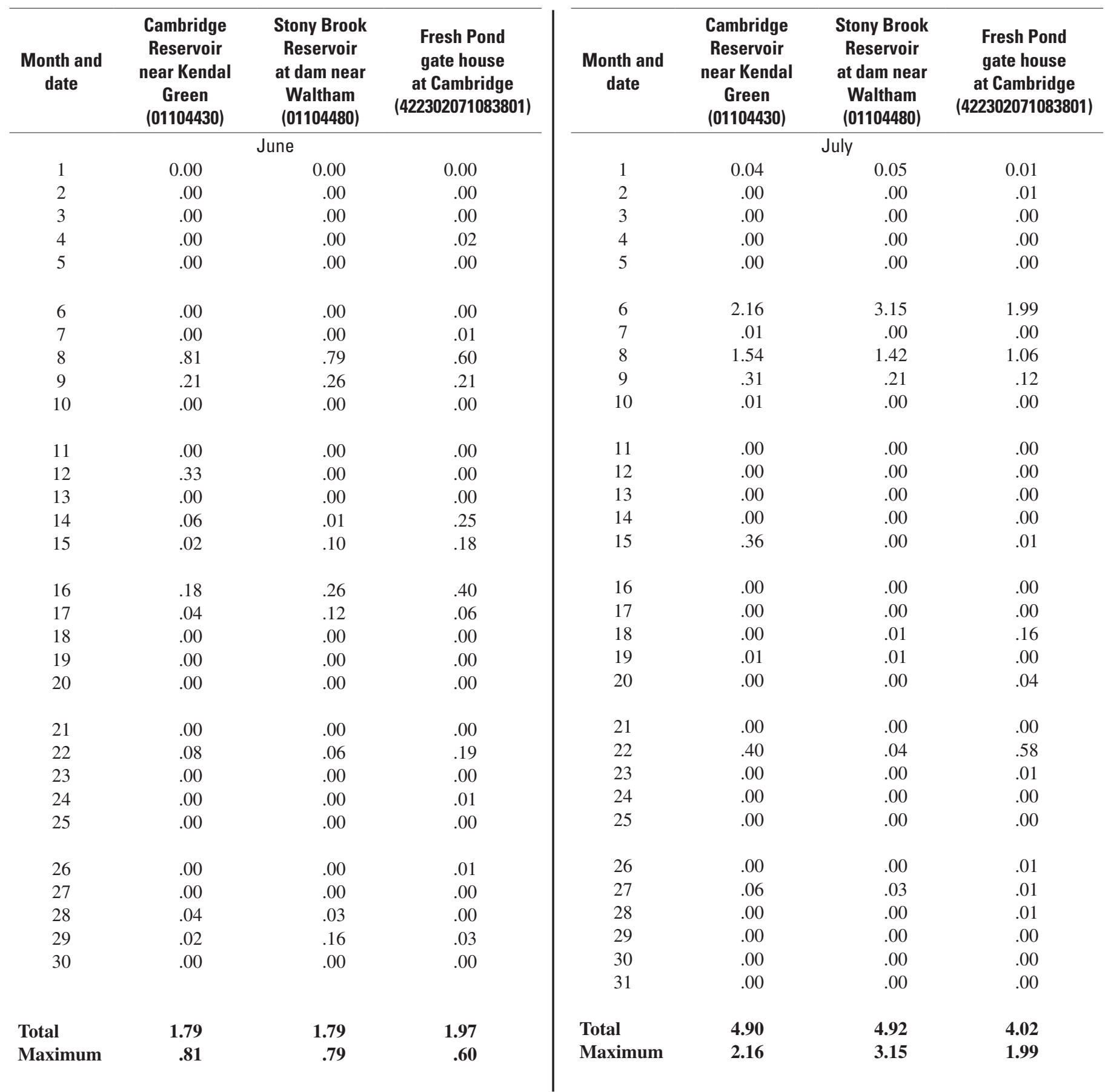


Table 11. Daily, monthly, and annual statistics for precipitation for U.S. Geological Survey stations 01104430 , Hobbs Brook below Cambridge Reservoir near Kendal Green; 01104480, Stony Brook Reservoir in Waltham; and 422302071083801, Fresh Pond Reservoir at Cambridge, Massachusetts, for water year 2005.-Continued

[Units are in inches; e, estimated]

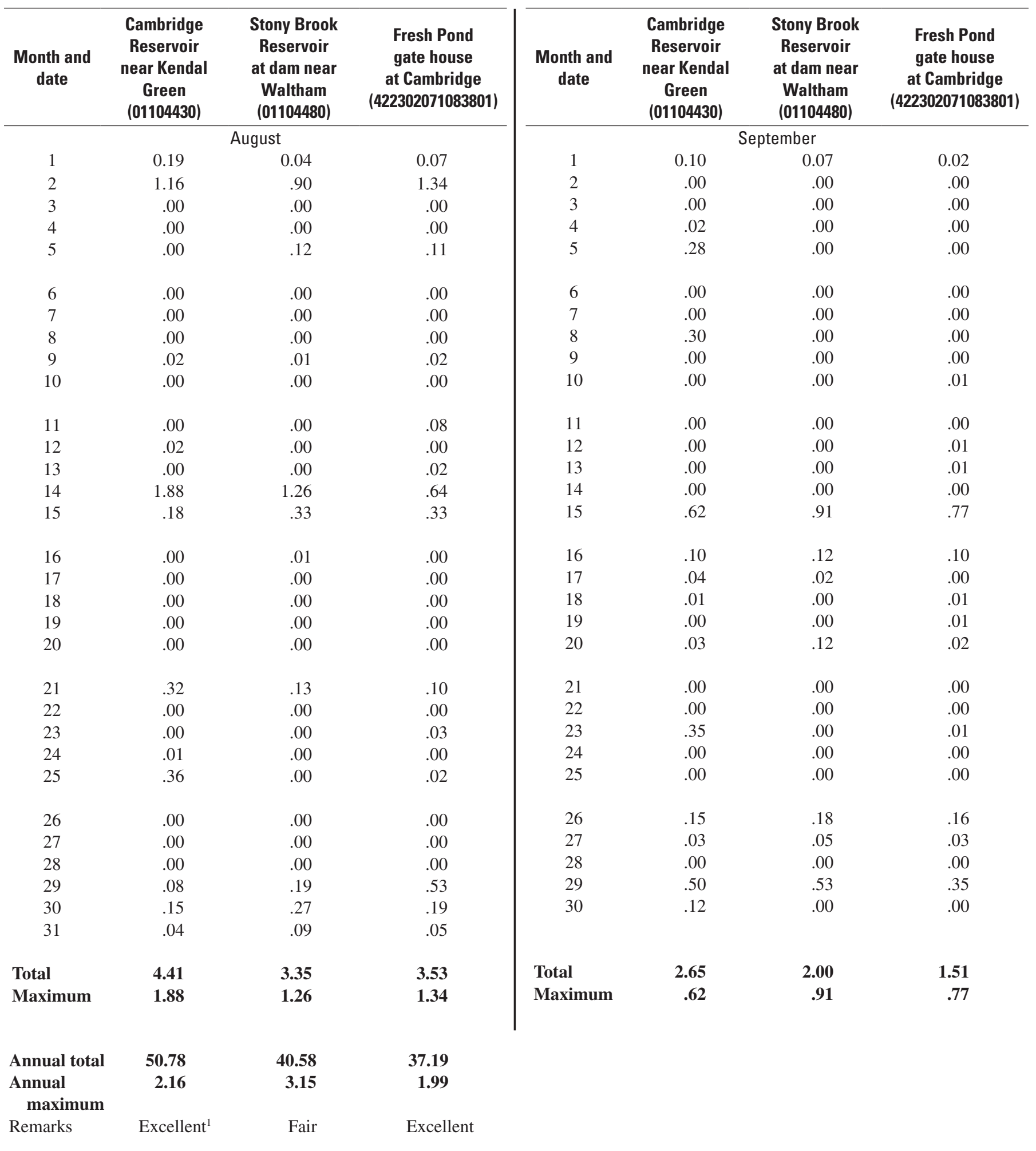

\footnotetext{
${ }^{1}$ Records that are estimated are rated as poor.
} 
Table 12. Daily, monthly, and annual statistics for air temperature for U.S. Geological Survey stations 01104430, Cambridge Reservoir near Kendal Green; 01104480, Stony Brook Reservoir in Waltham; and 422302071083801, Fresh Pond Reservoir at Cambridge, Massachusetts, for water year 2005.

[Units are in degrees Celsius. Max, maximum; Min, minimum; e, estimated]

\begin{tabular}{|c|c|c|c|c|c|c|c|c|c|}
\hline \multirow{2}{*}{$\begin{array}{l}\text { Month } \\
\text { and } \\
\text { date }\end{array}$} & \multicolumn{3}{|c|}{$\begin{array}{c}\text { Cambridge Reservoir } \\
\text { near Kendal Green } \\
\text { (01104430) }\end{array}$} & \multicolumn{3}{|c|}{$\begin{array}{c}\text { Stony Brook Reservoir at } \\
\text { dam near Waltham } \\
(01104480)\end{array}$} & \multicolumn{3}{|c|}{$\begin{array}{c}\text { Fresh Pond gate house } \\
\text { at Cambridge } \\
\text { (422302071083801) }\end{array}$} \\
\hline & Max & Min & Mean & Max & Min & Mean & Max & Min & Mean \\
\hline \multicolumn{10}{|c|}{ October } \\
\hline 1 & 20.8 & 11.5 & 15.2 & 21.6 & 11.4 & 15.3 & 22.6 & 12.6 & 16.2 \\
\hline 2 & 19.2 & 11.9 & 15.0 & 19.6 & 11.6 & 14.8 & 20.1 & 12.4 & 15.7 \\
\hline 3 & 16.6 & 6.9 & 12.7 & 17.1 & 7.4 & 12.8 & 17.0 & 8.6 & 13.6 \\
\hline 4 & 19.3 & 5.0 & 11.9 & 19.8 & 4.9 & 12.5 & 20.4 & 6.8 & 13.9 \\
\hline 5 & 13.9 & 2.9 & 9.8 & 14.3 & 2.9 & 9.9 & 15.4 & 5.5 & 11.0 \\
\hline 6 & 16.1 & .3 & 7.7 & 16.8 & 1.6 & 8.8 & 17.3 & 2.6 & 10.1 \\
\hline 7 & 20.4 & 4.7 & 12.0 & 23.0 & 5.1 & 12.9 & 23.2 & 7.3 & 14.1 \\
\hline 8 & 23.4 & 7.3 & 14.8 & 24.1 & 8.0 & 15.6 & 24.9 & 9.8 & 16.9 \\
\hline 9 & 21.6 & 12.5 & 16.3 & 21.3 & 11.9 & 16.2 & 22.4 & 13.1 & 17.3 \\
\hline 10 & 17.9 & 10.6 & 15.1 & 18.2 & 11.0 & 15.1 & 18.5 & 11.6 & 15.8 \\
\hline 11 & 16.6 & 9.6 & 12.5 & 16.3 & 9.8 & 12.4 & 17.1 & 10.7 & 13.3 \\
\hline 12 & 16.1 & 8.3 & 11.6 & 16.4 & 9.5 & 11.8 & 16.4 & 10.0 & 12.2 \\
\hline 13 & 19.8 & 3.8 & 10.8 & 22.0 & 5.3 & 12.2 & 21.5 & 7.4 & 13.4 \\
\hline 14 & 15.8 & 5.5 & 11.3 & 15.4 & 6.7 & 11.4 & 16.1 & 7.9 & 12.4 \\
\hline 15 & 14.9 & 12.9 & 13.7 & 14.5 & 12.7 & 13.4 & 15.3 & 13.1 & 13.9 \\
\hline 16 & 17.0 & 9.9 & 13.7 & 16.9 & 9.9 & 13.7 & 17.6 & 11.3 & 14.7 \\
\hline 17 & 13.6 & 5.6 & 9.5 & 13.1 & 6.6 & 9.8 & 13.6 & 7.7 & 10.8 \\
\hline 18 & 13.9 & 3.8 & 8.4 & 13.4 & 4.7 & 9.2 & 13.9 & 6.1 & 10.1 \\
\hline 19 & 9.4 & 7.0 & 8.4 & 9.2 & 7.2 & 8.2 & 10.3 & 7.8 & 8.9 \\
\hline 20 & 10.1 & 5.6 & 7.9 & 10.3 & 5.9 & 7.9 & 10.9 & 6.6 & 8.6 \\
\hline 21 & 10.0 & 5.0 & 7.6 & 10.1 & 5.4 & 7.7 & 10.6 & 5.7 & 8.2 \\
\hline 22 & 10.0 & 7.2 & 8.4 & 10.0 & 7.1 & 8.3 & 10.6 & 7.4 & 8.9 \\
\hline 23 & 9.8 & 6.2 & 8.2 & 9.6 & 6.3 & 8.0 & 10.5 & 6.7 & 8.6 \\
\hline 24 & 9.4 & 7.8 & 8.3 & 9.2 & 7.2 & 8.0 & 10.4 & 7.6 & 8.7 \\
\hline 25 & 11.1 & 6.6 & 8.7 & 10.8 & 6.9 & 8.6 & 11.6 & 7.4 & 9.2 \\
\hline 26 & 13.0 & 3.3 & 7.3 & 13.0 & 3.4 & 7.7 & 13.5 & 5.2 & 8.8 \\
\hline 27 & 14.7 & 2.8 & 9.5 & 14.2 & 5.1 & 9.6 & 14.7 & 5.5 & 10.6 \\
\hline 28 & 11.6 & .0 & 6.0 & 12.1 & 1.4 & 6.5 & 11.9 & 2.8 & 7.5 \\
\hline 29 & 14.5 & .0 & 7.0 & 16.0 & .9 & 7.5 & 14.9 & 2.8 & 8.8 \\
\hline 30 & 15.6 & 4.2 & 10.1 & 15.5 & 4.7 & 9.9 & 16.0 & 5.8 & 10.9 \\
\hline 31 & 21.1 & 12.2 & 16.5 & 19.7 & 13.1 & 16.6 & 20.2 & 13.3 & 70.0 \\
\hline Month & 23.4 & .0 & 10.8 & 24.1 & .9 & 11.0 & 24.9 & 2.6 & 11.9 \\
\hline
\end{tabular}


Table 12. Daily, monthly, and annual statistics for air temperature for U.S. Geological Survey stations 01104430, Cambridge Reservoir near Kendal Green; 01104480, Stony Brook Reservoir in Waltham; and 422302071083801, Fresh Pond Reservoir at Cambridge, Massachusetts, for water year 2005.-Continued

[Units are in degrees Celsius. Max, maximum; Min, minimum; e, estimated]

\begin{tabular}{|c|c|c|c|c|c|c|c|c|c|}
\hline \multirow{2}{*}{$\begin{array}{l}\text { Month } \\
\text { and } \\
\text { date }\end{array}$} & \multicolumn{3}{|c|}{$\begin{array}{c}\text { Cambridge Reservoir } \\
\text { near Kendal Green } \\
(01104430)\end{array}$} & \multicolumn{3}{|c|}{$\begin{array}{c}\text { Stony Brook Reservoir at } \\
\text { dam near Waltham } \\
(01104480)\end{array}$} & \multicolumn{3}{|c|}{$\begin{array}{c}\text { Fresh Pond gate house } \\
\text { at Cambridge } \\
(422302071083801)\end{array}$} \\
\hline & Max & Min & Mean & Max & Min & Mean & Max & Min & Mean \\
\hline \multicolumn{10}{|c|}{ November } \\
\hline 1 & 14.8 & 3.5 & 11.0 & 14.0 & 5.4 & 11.2 & 14.6 & 5.8 & 11.4 \\
\hline 2 & 11.3 & 1.0 & 7.3 & 11.6 & 2.2 & 7.8 & 12.5 & 3.4 & 8.8 \\
\hline 3 & 12.4 & 2.1 & 9.1 & 12.6 & 2.0 & 9.2 & 13.1 & 3.1 & 9.2 \\
\hline 4 & 9.2 & -2.3 & 4.6 & 8.9 & -1.4 & 4.7 & 10.0 & .2 & 6.2 \\
\hline 5 & 10.8 & 5.6 & 8.1 & 10.4 & 6.2 & 8.2 & 11.0 & 5.6 & 8.7 \\
\hline 6 & 12.9 & 3.1 & 9.0 & 12.9 & 3.5 & 9.2 & 13.4 & 5.2 & 9.9 \\
\hline 7 & 19.1 & 4.2 & 12.6 & 18.8 & 6.5 & 13.5 & 19.0 & 7.8 & 14.2 \\
\hline 8 & 14.4 & 1.6 & 7.2 & 14.8 & 2.0 & 7.3 & 15.0 & 1.6 & 7.3 \\
\hline 9 & 3.5 & -3.9 & -.1 & 3.4 & -2.8 & .2 & 3.9 & -2.2 & .8 \\
\hline 10 & 4.4 & -5.9 & -.7 & 4.5 & -5.5 & -.3 & 4.6 & -4.3 & .8 \\
\hline 11 & 14.2 & 1.1 & 7.4 & 14.0 & 1.0 & 8.1 & 14.3 & 3.1 & 9.0 \\
\hline 12 & 5.8 & -1.1 & 1.7 & 6.1 & -1.1 & 1.8 & 5.9 & -.9 & 2.2 \\
\hline 13 & 4.6 & -2.2 & .0 & 5.1 & -2.0 & .2 & 5.2 & -1.9 & .7 \\
\hline 14 & 8.2 & -8.3 & .1 & 8.7 & -5.0 & 1.4 & 8.9 & -4.1 & 2.5 \\
\hline 15 & 12.9 & -3.2 & 3.9 & 13.5 & -3.1 & 4.5 & 13.4 & .7 & 7.4 \\
\hline 16 & 7.7 & -1.5 & 2.8 & 8.0 & .5 & 3.7 & 8.5 & 1.9 & 5.6 \\
\hline 17 & 10.0 & -1.6 & 3.7 & 10.6 & -.6 & 4.5 & 10.6 & .3 & 6.2 \\
\hline 18 & 15.2 & 4.3 & 8.9 & 14.9 & 4.9 & 9.7 & 15.0 & 7.0 & 10.9 \\
\hline 19 & 16.1 & 1.3 & 9.6 & 15.4 & 3.5 & 10.1 & 15.8 & 6.4 & 11.6 \\
\hline 20 & 8.6 & -1.1 & 4.4 & 9.0 & .4 & 4.8 & 9.1 & 2.7 & 6.1 \\
\hline 21 & 6.9 & 4.6 & 6.0 & 6.9 & 4.6 & 5.9 & 7.6 & 5.2 & 6.6 \\
\hline 22 & 10.3 & -.8 & 5.3 & 9.7 & .8 & 6.3 & 10.4 & 2.6 & 7.6 \\
\hline 23 & 11.4 & -3.1 & 2.5 & 11.5 & -1.4 & 4.3 & 12.2 & .0 & 5.4 \\
\hline 24 & 15.2 & 1.5 & 8.2 & 15.1 & 2.8 & 8.3 & 16.1 & 5.5 & 9.7 \\
\hline 25 & 18.5 & 2.8 & 13.7 & 18.4 & 3.0 & 13.7 & 18.4 & 4.0 & 14.4 \\
\hline 26 & 4.4 & -4.0 & .3 & 4.1 & -2.0 & 1.0 & 4.7 & -.3 & 2.1 \\
\hline 27 & 9.7 & -5.0 & 2.2 & 9.5 & -3.7 & 3.1 & 10.3 & -1.7 & 4.6 \\
\hline 28 & 13.8 & 3.9 & 9.1 & 14.0 & 1.9 & 8.7 & 13.9 & 6.8 & 10.0 \\
\hline 29 & 9.8 & -.4 & 5.5 & 9.6 & .5 & 5.8 & 10.1 & 2.8 & 6.7 \\
\hline 30 & 8.7 & .6 & 4.3 & 8.4 & .5 & 4.7 & 9.1 & 2.6 & 5.8 \\
\hline Month & 19.1 & -8.3 & 5.6 & 18.8 & -5.5 & 6.1 & 19.0 & -4.3 & 7.1 \\
\hline
\end{tabular}


Table 12. Daily, monthly, and annual statistics for air temperature for U.S. Geological Survey stations 01104430, Cambridge Reservoir near Kendal Green; 01104480, Stony Brook Reservoir in Waltham; and 422302071083801, Fresh Pond Reservoir at Cambridge, Massachusetts, for water year 2005.-Continued

[Units are in degrees Celsius. Max, maximum; Min, minimum; e, estimated]

\begin{tabular}{|c|c|c|c|c|c|c|c|c|c|}
\hline \multirow{2}{*}{$\begin{array}{l}\text { Month } \\
\text { and } \\
\text { date }\end{array}$} & \multicolumn{3}{|c|}{$\begin{array}{c}\text { Cambridge Reservoir } \\
\text { near Kendal Green } \\
\text { (01104430) }\end{array}$} & \multicolumn{3}{|c|}{$\begin{array}{c}\text { Stony Brook Reservoir at } \\
\text { dam near Waltham } \\
(01104480)\end{array}$} & \multicolumn{3}{|c|}{$\begin{array}{l}\text { Fresh Pond gate house } \\
\text { at Cambridge } \\
\text { (422302071083801) }\end{array}$} \\
\hline & Max & Min & Mean & Max & Min & Mean & Max & Min & Mean \\
\hline \multicolumn{10}{|c|}{ December } \\
\hline 1 & 13.9 & 3.7 & 8.8 & 13.9 & 4.7 & 8.9 & 14.7 & 6.6 & 9.7 \\
\hline 2 & 8.4 & -1.6 & 3.5 & 7.7 & .2 & 4.7 & 8.2 & 2.4 & 5.6 \\
\hline 3 & 7.9 & -1.5 & 2.9 & 7.3 & -.8 & 3.1 & 7.7 & 1.5 & 4.3 \\
\hline 4 & 4.0 & -3.5 & .6 & 3.7 & -2.2 & 1.1 & 4.5 & -.7 & 1.9 \\
\hline 5 & 10.2 & -2.3 & 5.0 & 9.8 & -1.8 & 5.2 & 10.0 & -1.5 & 5.5 \\
\hline 6 & -1.2 & -5.1 & -3.0 & -1.2 & -4.8 & -2.7 & -.6 & -4.2 & -2.2 \\
\hline 7 & 7.6 & -2.7 & 2.6 & 7.6 & -2.5 & 3.1 & 8.4 & -1.6 & 4.8 \\
\hline 8 & 11.3 & 1.2 & 5.6 & 10.9 & 1.2 & 6.0 & 11.3 & 2.0 & 6.5 \\
\hline 9 & 5.6 & -1.1 & 2.8 & 5.5 & .4 & 3.6 & 6.6 & 2.3 & 4.8 \\
\hline 10 & 7.0 & 3.9 & 5.6 & 6.9 & 4.0 & 5.5 & 7.5 & 5.5 & 6.2 \\
\hline 11 & 7.0 & 3.0 & 5.1 & 7.0 & 3.5 & 5.3 & 7.7 & 4.0 & 6.1 \\
\hline 12 & 5.9 & 1.3 & 3.5 & 6.2 & 1.6 & 3.9 & 7.1 & 2.8 & 4.6 \\
\hline 13 & 8.7 & 1.6 & 4.3 & 8.2 & 1.7 & 4.5 & 8.5 & 2.8 & 5.4 \\
\hline 14 & 1.8 & -6.8 & -2.3 & 2.4 & -5.9 & -1.9 & 2.8 & -5.8 & -1.4 \\
\hline 15 & -2.7 & -9.3 & -6.3 & -2.6 & -8.2 & -5.9 & -2.3 & -7.7 & -5.2 \\
\hline 16 & 5.5 & -10.1 & -2.6 & 5.7 & -9.7 & -1.9 & 6.1 & -7.4 & -.7 \\
\hline 17 & 5.8 & -6.1 & 1.5 & 5.1 & -4.4 & 1.8 & 5.5 & -2.6 & 2.5 \\
\hline 18 & 2.9 & -9.3 & -3.0 & 2.7 & -8.3 & -2.7 & 2.9 & -6.0 & -1.3 \\
\hline 19 & 5.1 & -3.1 & .6 & 5.0 & -3.5 & .8 & 5.8 & -2.0 & 2.0 \\
\hline 20 & 1.5 & -15.2 & -8.4 & 1.2 & -14.7 & -8.1 & 3.0 & -13.8 & -7.3 \\
\hline 21 & -1.5 & -16.4 & -9.4 & -.9 & -15.6 & -8.3 & -.4 & -14.8 & -7.3 \\
\hline 22 & 8.0 & -9.8 & -1.1 & 8.8 & -8.0 & 1.1 & 9.8 & -4.9 & 2.9 \\
\hline 23 & 16.7 & 1.0 & 9.9 & 16.6 & 1.8 & 9.9 & 17.1 & 3.3 & 11.4 \\
\hline 24 & 10.6 & -2.9 & .7 & 10.7 & -2.4 & .9 & 11.4 & -2.0 & 1.4 \\
\hline 25 & -.6 & -8.1 & -3.8 & -.8 & -7.3 & -3.6 & .0 & -5.5 & -2.6 \\
\hline 26 & -4.3 & -6.4 & -5.2 & -4.1 & -6.0 & -4.9 & -3.0 & -5.9 & -4.4 \\
\hline 27 & -4.4 & -12.8 & -7.5 & -4.0 & -11.1 & -6.8 & -3.4 & -10.4 & -6.4 \\
\hline 28 & -2.3 & -16.5 & -7.3 & -2.3 & -13.0 & -6.6 & -2.1 & -12.4 & -6.1 \\
\hline 29 & 5.1 & -2.7 & .5 & 4.8 & -2.5 & .9 & 5.1 & -2.1 & 1.5 \\
\hline 30 & 3.4 & -6.3 & -.7 & 3.6 & -4.7 & .0 & 4.5 & -2.2 & 1.1 \\
\hline 31 & 8.2 & -6.4 & 2.6 & 8.3 & -5.0 & 2.9 & 8.8 & -3.0 & 4.5 \\
\hline Month & 16.7 & -16.5 & .2 & 16.6 & -15.6 & .6 & 17.1 & -14.8 & 1.5 \\
\hline
\end{tabular}


Table 12. Daily, monthly, and annual statistics for air temperature for U.S. Geological Survey stations 01104430, Cambridge Reservoir near Kendal Green; 01104480, Stony Brook Reservoir in Waltham; and 422302071083801, Fresh Pond Reservoir at Cambridge, Massachusetts, for water year 2005.-Continued

[Units are in degrees Celsius. Max, maximum; Min, minimum; e, estimated]

\begin{tabular}{|c|c|c|c|c|c|c|c|c|c|}
\hline \multirow{2}{*}{$\begin{array}{l}\text { Month } \\
\text { and } \\
\text { date }\end{array}$} & \multicolumn{3}{|c|}{$\begin{array}{c}\text { Cambridge Reservoir } \\
\text { near Kendal Green } \\
(01104430)\end{array}$} & \multicolumn{3}{|c|}{$\begin{array}{c}\text { Stony Brook Reservoir at } \\
\text { dam near Waltham } \\
(01104480)\end{array}$} & \multicolumn{3}{|c|}{$\begin{array}{c}\text { Fresh Pond gate house } \\
\text { at Cambridge } \\
(422302071083801)\end{array}$} \\
\hline & Max & Min & Mean & Max & Min & Mean & Max & Min & Mean \\
\hline \multicolumn{10}{|c|}{ January } \\
\hline 1 & 11.0 & -0.3 & 5.8 & 11.1 & 0.9 & 6.5 & 11.4 & 1.5 & 7.2 \\
\hline 2 & 3.0 & -2.9 & .0 & 3.3 & -2.3 & .3 & 4.5 & -2.3 & .7 \\
\hline 3 & 11.8 & 3.0 & 7.9 & 11.4 & 3.3 & 8.3 & 11.6 & 4.5 & 8.9 \\
\hline 4 & 7.0 & 1.0 & 4.7 & 6.9 & 2.6 & 5.2 & 7.6 & 3.6 & 5.5 \\
\hline 5 & 1.7 & -3.0 & -.6 & 2.7 & -2.7 & -.3 & 3.7 & -2.3 & .3 \\
\hline 6 & 2.0 & -4.8 & -1.6 & 2.0 & -4.7 & -1.5 & 3.1 & -4.3 & -.6 \\
\hline 7 & 3.7 & -4.8 & .1 & 3.4 & -3.4 & .6 & 3.8 & -1.5 & 1.3 \\
\hline 8 & -.2 & -4.8 & -1.7 & -.3 & -3.1 & -1.5 & 1.1 & -1.8 & -.6 \\
\hline 9 & .0 & -7.0 & -2.4 & -.2 & -5.1 & -2.2 & .3 & -4.1 & -1.4 \\
\hline 10 & 4.2 & -1.2 & 1.4 & 3.9 & -1.2 & 1.5 & 4.4 & -.5 & 2.1 \\
\hline 11 & 1.2 & -3.6 & -.6 & 2.0 & -2.5 & -.4 & 1.9 & -1.2 & .4 \\
\hline 12 & 2.8 & -1.7 & .9 & 2.8 & -1.9 & .9 & 3.4 & -.8 & 1.7 \\
\hline 13 & 13.4 & -.6 & 3.3 & 14.0 & -.8 & 3.3 & 14.8 & -.6 & 3.9 \\
\hline 14 & 16.7 & -.2 & 8.1 & 16.9 & .0 & 8.3 & 18.1 & .3 & 9.0 \\
\hline 15 & -.2 & -5.7 & -3.0 & .0 & -5.3 & -2.8 & .3 & -4.1 & -2.1 \\
\hline 16 & -2.4 & -7.3 & -4.7 & -2.3 & -6.6 & -4.6 & -1.5 & -5.8 & -3.9 \\
\hline 17 & -4.4 & -11.5 & -7.0 & -4.6 & -11.0 & -6.7 & -3.9 & -10.8 & -6.4 \\
\hline 18 & -11.0 & -16.8 & -13.8 & -10.4 & -16.2 & -13.2 & -10.4 & -15.0 & -12.7 \\
\hline 19 & -4.4 & -18.2 & -10.7 & -4.6 & -17.6 & -10.5 & -3.5 & -16.2 & -9.6 \\
\hline 20 & -2.1 & -10.0 & -6.3 & -2.9 & -9.6 & -6.3 & -2.3 & -9.9 & -5.8 \\
\hline 21 & -10.0 & -18.1 & -14.8 & -9.6 & -17.5 & -14.5 & -9.9 & -16.6 & -13.9 \\
\hline 22 & -4.3 & -21.0 & -14.0 & -3.9 & -20.1 & -13.5 & -3.3 & -19.1 & -12.5 \\
\hline 23 & -4.4 & -15.9 & -11.7 & -4.2 & -15.4 & -11.1 & -3.7 & -15.0 & -10.7 \\
\hline 24 & -4.5 & -20.3 & -11.9 & -5.0 & -17.0 & -11.0 & -4.4 & -15.2 & -10.1 \\
\hline 25 & -3.2 & -13.3 & -6.9 & -3.5 & -9.3 & -6.4 & -3.0 & -8.8 & -6.1 \\
\hline 26 & -5.9 & -9.5 & -7.6 & -5.5 & -9.3 & -7.4 & -4.8 & -9.3 & -6.9 \\
\hline 27 & -9.2 & -15.6 & -12.0 & -9.1 & -15.3 & -11.8 & -8.6 & -14.9 & -11.5 \\
\hline 28 & -6.1 & -17.8 & -12.9 & -5.8 & -18.0 & -13.0 & -5.8 & -16.8 & -11.4 \\
\hline 29 & 3.0 & -19.8 & -8.4 & 2.9 & -20.4 & -8.0 & 2.7 & -16.4 & -6.1 \\
\hline 30 & 3.2 & -9.6 & -2.5 & 4.5 & -5.7 & -1.8 & 4.2 & -5.4 & -.9 \\
\hline 31 & -1.5 & -13.5 & -6.0 & -1.1 & -13.1 & -5.3 & -1.2 & -11.2 & -5.0 \\
\hline Month & 16.7 & -21.0 & -4.2 & 16.9 & -20.4 & -3.8 & 18.1 & -19.1 & -3.1 \\
\hline
\end{tabular}


Table 12. Daily, monthly, and annual statistics for air temperature for U.S. Geological Survey stations 01104430, Cambridge Reservoir near Kendal Green; 01104480, Stony Brook Reservoir in Waltham; and 422302071083801, Fresh Pond Reservoir at Cambridge, Massachusetts, for water year 2005.-Continued

[Units are in degrees Celsius. Max, maximum; Min, minimum; e, estimated]

\begin{tabular}{|c|c|c|c|c|c|c|c|c|c|}
\hline \multirow{2}{*}{$\begin{array}{l}\text { Month } \\
\text { and } \\
\text { date }\end{array}$} & \multicolumn{3}{|c|}{$\begin{array}{l}\text { Cambridge Reservoir } \\
\text { near Kendal Green } \\
\text { (01104430) }\end{array}$} & \multicolumn{3}{|c|}{$\begin{array}{l}\text { Stony Brook Reservoir at } \\
\text { dam near Waltham } \\
\text { (01104480) }\end{array}$} & \multicolumn{3}{|c|}{$\begin{array}{l}\text { Fresh Pond gate house } \\
\text { at Cambridge } \\
\text { (422302071083801) }\end{array}$} \\
\hline & Max & Min & Mean & Max & Min & Mean & Max & Min & Mean \\
\hline \multicolumn{10}{|c|}{ February } \\
\hline 1 & 3.0 & -16.0 & -7.6 & 4.4 & -16.4 & -6.7 & 3.8 & -13.7 & -5.9 \\
\hline 2 & 2.7 & -14.5 & -5.5 & 3.8 & -13.8 & -4.8 & 3.4 & -12.1 & -3.9 \\
\hline 3 & 3.4 & -9.4 & -2.1 & 4.0 & -7.8 & -1.7 & 3.7 & -5.8 & -.9 \\
\hline 4 & 2.1 & -3.1 & .5 & 2.0 & -2.5 & .6 & 2.6 & -2.2 & 1.0 \\
\hline 5 & 11.5 & -5.0 & 1.8 & 12.0 & -4.8 & 2.2 & 12.7 & -1.9 & 3.5 \\
\hline 6 & 6.6 & -4.6 & .7 & 8.2 & -3.6 & 1.5 & 7.1 & -3.5 & 2.3 \\
\hline 7 & 11.2 & -6.5 & .4 & 11.6 & -5.6 & 1.5 & 12.1 & -5.0 & 2.0 \\
\hline 8 & 10.0 & -3.7 & 2.0 & 11.2 & -3.2 & 3.3 & 11.5 & -1.4 & 5.2 \\
\hline 9 & 10.2 & -1.4 & 4.3 & 11.1 & -.8 & 5.0 & 11.0 & 1.1 & 6.5 \\
\hline 10 & 4.0 & .0 & 2.5 & 4.2 & -.3 & 2.3 & 5.7 & .6 & 3.0 \\
\hline 11 & .0 & -4.7 & -2.6 & -.1 & -4.7 & -2.4 & .8 & -3.5 & -1.6 \\
\hline 12 & 4.1 & -7.2 & -1.6 & 3.1 & -4.5 & -1.0 & 3.9 & -3.1 & .1 \\
\hline 13 & 2.7 & -5.3 & -1.4 & 2.4 & -5.1 & -1.5 & 2.7 & -4.5 & -.6 \\
\hline 14 & 4.2 & -9.3 & -1.6 & 3.9 & -8.7 & -1.6 & 4.8 & -7.0 & -.5 \\
\hline 15 & 10.4 & -.3 & 5.8 & 10.2 & .9 & 6.2 & 10.5 & 2.5 & 7.3 \\
\hline 16 & 12.7 & -1.1 & 5.1 & 12.4 & -1.0 & 5.1 & 13.0 & .9 & 6.6 \\
\hline 17 & 3.9 & -2.4 & .9 & 3.7 & -2.4 & .9 & 4.3 & -1.0 & 1.6 \\
\hline 18 & 1.1 & -8.3 & -3.2 & -.4 & -8.3 & -3.4 & .2 & -7.1 & -2.7 \\
\hline 19 & -2.5 & -11.4 & -7.4 & -2.8 & -11.4 & -7.5 & -2.5 & -10.8 & -6.6 \\
\hline 20 & -.9 & -7.8 & -4.5 & -1.2 & -8.0 & -4.7 & -1.0 & -6.4 & -4.0 \\
\hline 21 & -.4 & -6.2 & -2.8 & -.5 & -5.3 & -2.8 & .5 & -4.9 & -2.0 \\
\hline 22 & -.1 & -5.6 & -3.0 & .0 & -5.4 & -3.0 & .1 & -5.2 & -2.6 \\
\hline 23 & 2.3 & -5.4 & -1.4 & 2.0 & -5.9 & -1.3 & 2.2 & -4.6 & -.5 \\
\hline 24 & -2.8 & -10.9 & -6.5 & -3.2 & -11.2 & -6.5 & -3.5 & -9.8 & -5.9 \\
\hline 25 & -1.9 & -11.4 & -5.7 & -2.4 & -9.1 & -5.5 & -1.3 & -7.5 & -4.8 \\
\hline 26 & 1.1 & -17.1 & -6.3 & .5 & -16.0 & -5.8 & 1.3 & -10.5 & -3.7 \\
\hline 27 & 1.0 & -9.8 & -4.6 & .6 & -8.4 & -4.2 & .9 & -7.2 & -3.0 \\
\hline 28 & 1.1 & -11.4 & -3.8 & 1.2 & -10.4 & -3.7 & 1.6 & -6.8 & -2.0 \\
\hline Month & 12.7 & -17.1 & -1.7 & 12.4 & -16.4 & -1.4 & 13.0 & -13.7 & -.4 \\
\hline
\end{tabular}


Table 12. Daily, monthly, and annual statistics for air temperature for U.S. Geological Survey stations 01104430, Cambridge Reservoir near Kendal Green; 01104480, Stony Brook Reservoir in Waltham; and 422302071083801, Fresh Pond Reservoir at Cambridge, Massachusetts, for water year 2005.-Continued

[Units are in degrees Celsius. Max, maximum; Min, minimum; e, estimated]

\begin{tabular}{|c|c|c|c|c|c|c|c|c|c|}
\hline \multirow{2}{*}{$\begin{array}{l}\text { Month } \\
\text { and } \\
\text { date }\end{array}$} & \multicolumn{3}{|c|}{$\begin{array}{l}\text { Cambridge Reservoir } \\
\text { near Kendal Green } \\
(01104430)\end{array}$} & \multicolumn{3}{|c|}{$\begin{array}{l}\text { Stony Brook Reservoir at } \\
\text { dam near Waltham } \\
(01104480)\end{array}$} & \multicolumn{3}{|c|}{$\begin{array}{c}\text { Fresh Pond gate house } \\
\text { at Cambridge } \\
(422302071083801)\end{array}$} \\
\hline & Max & Min & Mean & Max & Min & Mean & Max & Min & Mean \\
\hline \multicolumn{10}{|c|}{ March } \\
\hline 1 & -1.0 & -4.3 & -2.4 & -0.9 & -4.3 & -2.4 & -0.5 & -3.7 & -1.7 \\
\hline 2 & 1.2 & -5.7 & -2.7 & .8 & -5.0 & -2.4 & 1.1 & -4.4 & -1.9 \\
\hline 3 & -1.6 & -8.8 & -5.0 & -2.0 & -7.5 & -4.9 & -1.6 & -6.6 & -4.2 \\
\hline 4 & 1.2 & -14.4 & -5.9 & .0 & -14.9 & -5.4 & .6 & -8.8 & -3.8 \\
\hline 5 & 5.4 & -11.0 & -2.5 & 5.2 & -7.0 & -.8 & 5.3 & -5.9 & -.3 \\
\hline 6 & 6.1 & -7.7 & .5 & 5.8 & -5.5 & 1.3 & 5.9 & -2.7 & 2.0 \\
\hline 7 & 9.8 & -.7 & 5.5 & 9.7 & 1.8 & 6.0 & 10.2 & 2.5 & 6.5 \\
\hline 8 & 7.1 & -9.8 & .6 & 7.2 & -9.6 & .9 & 8.1 & -8.5 & 1.8 \\
\hline 9 & -5.1 & -12.8 & -8.7 & -5.6 & -12.6 & -8.6 & -4.9 & -11.6 & -7.8 \\
\hline 10 & -.7 & -16.4 & -8.4 & -1.2 & -14.1 & -7.3 & -.8 & -10.3 & -5.6 \\
\hline 11 & 1.1 & -12.7 & -4.0 & .5 & -11.2 & -3.9 & 1.4 & -7.9 & -2.1 \\
\hline 12 & .7 & -1.2 & .3 & .7 & -1.3 & .1 & 1.5 & -.6 & .7 \\
\hline 13 & 4.2 & -5.3 & -.3 & 4.2 & -5.2 & -.1 & 4.3 & -2.5 & .6 \\
\hline 14 & 5.1 & -4.7 & .3 & 4.3 & -4.5 & .1 & 4.8 & -3.5 & .9 \\
\hline 15 & 7.3 & -2.3 & 2.1 & 7.1 & -2.2 & 2.0 & 7.4 & -1.1 & 2.7 \\
\hline 16 & 6.5 & -2.8 & 1.8 & 6.0 & -2.2 & 1.9 & 6.5 & -.5 & 2.8 \\
\hline 17 & 7.5 & -6.5 & .4 & 7.3 & -6.4 & .6 & 7.4 & -2.6 & 2.0 \\
\hline 18 & 6.5 & -4.6 & 1.2 & 6.1 & -4.2 & 1.0 & 6.6 & -2.5 & 2.2 \\
\hline 19 & 8.6 & -4.2 & 1.6 & 8.0 & -2.6 & 1.9 & 8.7 & -1.4 & 3.3 \\
\hline 20 & 10.6 & -5.5 & 2.9 & 9.2 & -5.3 & 2.7 & 9.9 & -1.9 & 4.6 \\
\hline 21 & 6.2 & -2.9 & 2.6 & 6.9 & -2.1 & 2.9 & 6.3 & -.6 & 3.7 \\
\hline 22 & 12.4 & -3.3 & 4.0 & 11.8 & -2.8 & 4.9 & 12.3 & -1.2 & 6.4 \\
\hline 23 & 6.0 & -1.4 & 2.7 & 6.3 & -1.0 & 2.8 & 7.1 & .8 & 4.0 \\
\hline 24 & 4.6 & -2.4 & 1.0 & 4.6 & -2.1 & 1.3 & 5.5 & -1.1 & 2.0 \\
\hline 25 & 9.5 & -2.4 & 4.3 & 8.4 & -2.2 & 3.7 & 8.8 & -.2 & 4.7 \\
\hline 26 & 5.6 & -2.9 & 1.3 & 5.6 & -2.1 & 1.3 & 4.9 & -1.6 & 1.8 \\
\hline 27 & 11.6 & -4.1 & 3.8 & 11.2 & -4.5 & 3.5 & 11.5 & -2.0 & 4.7 \\
\hline 28 & 5.4 & .9 & 3.5 & 5.6 & 1.1 & 3.6 & 6.3 & 2.3 & 4.3 \\
\hline 29 & 10.3 & 3.6 & 6.7 & 10.7 & 3.4 & 6.3 & 11.0 & 4.2 & 7.5 \\
\hline 30 & 13.2 & .7 & 6.5 & 12.6 & 1.0 & 6.1 & 11.4 & 2.1 & 7.0 \\
\hline 31 & 10.0 & -.3 & 5.0 & 9.6 & -.6 & 5.0 & 9.4 & 1.2 & 5.7 \\
\hline Month & 13.2 & -16.4 & .60 & 12.6 & -14.9 & .8 & 12.3 & -11.6 & 1.8 \\
\hline
\end{tabular}


Table 12. Daily, monthly, and annual statistics for air temperature for U.S. Geological Survey stations 01104430, Cambridge Reservoir near Kendal Green; 01104480, Stony Brook Reservoir in Waltham; and 422302071083801, Fresh Pond Reservoir at Cambridge, Massachusetts, for water year 2005.-Continued

[Units are in degrees Celsius. Max, maximum; Min, minimum; e, estimated]

\begin{tabular}{|c|c|c|c|c|c|c|c|c|c|}
\hline \multirow{2}{*}{$\begin{array}{l}\text { Month } \\
\text { and } \\
\text { date }\end{array}$} & \multicolumn{3}{|c|}{$\begin{array}{l}\text { Cambridge Reservoir } \\
\text { near Kendal Green } \\
\text { (01104430) }\end{array}$} & \multicolumn{3}{|c|}{$\begin{array}{l}\text { Stony Brook Reservoir at } \\
\text { dam near Waltham } \\
(01104480)\end{array}$} & \multicolumn{3}{|c|}{$\begin{array}{c}\text { Fresh Pond gate house } \\
\text { at Cambridge } \\
(422302071083801)\end{array}$} \\
\hline & Max & Min & Mean & Max & Min & Mean & Max & Min & Mean \\
\hline \multicolumn{10}{|c|}{ April } \\
\hline 1 & 13.7 & 3.2 & 7.2 & 13.0 & 3.4 & 7.3 & 11.2 & 4.0 & 7.4 \\
\hline 2 & 10.2 & 3.0 & 5.9 & 10.7 & 3.1 & 6.0 & 10.5 & 3.4 & 6.3 \\
\hline 3 & 12.5 & 5.4 & 9.2 & 12.2 & 5.4 & 9.2 & 12.7 & 6.5 & 10.0 \\
\hline 4 & 11.6 & 3.5 & 6.8 & 10.6 & 3.6 & 6.7 & 11.0 & 4.5 & 7.3 \\
\hline 5 & 17.1 & .2 & 9.0 & 16.4 & 1.1 & 9.0 & 16.9 & 3.2 & 9.7 \\
\hline 6 & 19.8 & 2.8 & 11.1 & 19.7 & 4.4 & 11.5 & 17.6 & 6.0 & 11.7 \\
\hline 7 & 20.0 & 6.3 & 13.2 & 21.3 & 7.3 & 13.4 & 19.1 & 8.0 & 12.6 \\
\hline 8 & 15.2 & 6.6 & 10.7 & 16.2 & 6.1 & 10.8 & 14.2 & 7.4 & 11.2 \\
\hline 9 & 14.9 & 2.7 & 9.0 & 14.5 & 3.5 & 8.7 & 12.8 & 5.3 & 9.1 \\
\hline 10 & 21.1 & .1 & 11.6 & 20.7 & 1.0 & 11.6 & 21.2 & 3.1 & 12.6 \\
\hline 11 & 11.8 & 3.7 & 6.9 & 11.9 & 4.2 & 6.6 & 13.4 & 4.8 & 7.0 \\
\hline 12 & 10.3 & -.8 & 3.6 & 9.7 & -.3 & 3.2 & 9.4 & .3 & 3.8 \\
\hline 13 & 10.9 & -1.6 & 4.3 & 11.7 & -.3 & 4.6 & 10.7 & .5 & 5.0 \\
\hline 14 & 13.0 & .4 & 7.2 & 11.8 & 1.5 & 7.0 & 11.8 & 4.1 & 7.8 \\
\hline 15 & 11.2 & -.6 & 5.7 & 9.5 & .8 & 5.8 & 10.4 & 1.9 & 6.2 \\
\hline 16 & 14.2 & -2.3 & 6.3 & 13.4 & -1.2 & 6.6 & 13.0 & .9 & 7.4 \\
\hline 17 & 23.9 & -.9 & 11.7 & 23.9 & .2 & 12.1 & 24.5 & 2.9 & 12.7 \\
\hline 18 & 21.4 & 6.2 & 14.1 & 21.0 & 8.1 & 14.4 & 21.4 & 11.2 & 16.1 \\
\hline 19 & 25.3 & 2.0 & 14.7 & 25.4 & 4.0 & 15.5 & 25.4 & 6.6 & 16.6 \\
\hline 20 & 30.3 & 10.4 & 20.9 & 29.0 & 11.0 & 21.7 & 29.9 & 11.5 & 22.0 \\
\hline 21 & 14.4 & 3.9 & 10.1 & 14.2 & 5.2 & 9.7 & 14.4 & 5.9 & 10.4 \\
\hline 22 & 17.0 & -.7 & 8.7 & 16.5 & .9 & 8.9 & 16.3 & 3.3 & 9.9 \\
\hline 23 & 14.9 & 5.8 & 10.6 & 15.8 & 6.4 & 11.0 & 16.8 & 6.9 & 11.8 \\
\hline 24 & 17.0 & 5.1 & 13.2 & 16.2 & 6.0 & 13.1 & 16.8 & 7.7 & 13.7 \\
\hline 25 & 10.9 & 3.5 & 7.4 & 11.2 & 4.9 & 7.8 & 10.9 & 5.3 & 8.5 \\
\hline 26 & 20.9 & 1.4 & 11.7 & 20.3 & 2.8 & 12.2 & 21.1 & 5.3 & 13.2 \\
\hline 27 & 12.5 & 9.6 & 11.3 & 12.5 & 9.8 & 11.3 & 13.6 & 9.6 & 11.5 \\
\hline 28 & 15.9 & 7.9 & 11.1 & 15.1 & 8.0 & 11.0 & 15.7 & 8.4 & 11.4 \\
\hline 29 & 17.6 & 3.6 & 11.3 & 17.6 & 5.3 & 11.2 & 18.1 & 5.9 & 11.8 \\
\hline 30 & 13.6 & 8.1 & 11.6 & 13.4 & 8.5 & 11.5 & 14.3 & 9.5 & 12.2 \\
\hline Month & 30.3 & -2.3 & 9.9 & 29.0 & -1.2 & 10.0 & 29.9 & .3 & 10.6 \\
\hline
\end{tabular}


Table 12. Daily, monthly, and annual statistics for air temperature for U.S. Geological Survey stations 01104430, Cambridge Reservoir near Kendal Green; 01104480, Stony Brook Reservoir in Waltham; and 422302071083801, Fresh Pond Reservoir at Cambridge, Massachusetts, for water year 2005.-Continued

[Units are in degrees Celsius. Max, maximum; Min, minimum; e, estimated]

\begin{tabular}{|c|c|c|c|c|c|c|c|c|c|}
\hline \multirow{2}{*}{$\begin{array}{l}\text { Month } \\
\text { and } \\
\text { date }\end{array}$} & \multicolumn{3}{|c|}{$\begin{array}{c}\text { Cambridge Reservoir } \\
\text { near Kendal Green } \\
\text { (01104430) }\end{array}$} & \multicolumn{3}{|c|}{$\begin{array}{c}\text { Stony Brook Reservoir at } \\
\text { dam near Waltham } \\
(01104480)\end{array}$} & \multicolumn{3}{|c|}{$\begin{array}{c}\text { Fresh Pond gate house } \\
\text { at Cambridge } \\
(422302071083801)\end{array}$} \\
\hline & Max & Min & Mean & Max & Min & Mean & Max & Min & Mean \\
\hline \multicolumn{10}{|c|}{ May } \\
\hline 1 & 14.2 & 6.6 & 12.3 & 14.1 & 8.8 & 12.6 & 14.7 & 9.5 & 13.4 \\
\hline 2 & 17.4 & 3.1 & 10.5 & 16.6 & 5.8 & 11.1 & 17.2 & 6.2 & 11.8 \\
\hline 3 & 15.1 & 3.8 & 9.4 & 13.8 & 4.6 & 9.5 & 14.3 & 5.6 & 10.3 \\
\hline 4 & 14.7 & 2.6 & 9.8 & 13.3 & 4.5 & 10.0 & 13.7 & 6.0 & 10.9 \\
\hline 5 & 15.7 & .6 & 9.3 & 14.5 & 3.0 & 9.6 & 14.7 & 3.8 & 10.5 \\
\hline 6 & 13.2 & 2.6 & 8.8 & 12.8 & 3.3 & 8.8 & 13.1 & 5.3 & 9.6 \\
\hline 7 & 9.8 & 5.6 & 7.0 & 9.3 & 5.5 & 6.6 & 9.7 & 5.9 & 7.1 \\
\hline 8 & 10.4 & 6.0 & 8.3 & 10.1 & 5.8 & 8.0 & 10.5 & 5.9 & 8.3 \\
\hline 9 & 14.1 & 8.3 & 10.4 & 13.6 & 7.9 & 10.0 & 13.6 & 8.3 & 10.2 \\
\hline 10 & 19.6 & 9.6 & 13.5 & 18.7 & 9.7 & 13.3 & 19.5 & 10.5 & 13.8 \\
\hline 11 & 26.5 & 8.0 & 16.7 & 25.0 & 7.9 & 16.1 & 26.6 & 9.7 & 17.2 \\
\hline 12 & 16.7 & 7.0 & 13.1 & 16.0 & 7.9 & 12.8 & 17.6 & 8.8 & 13.6 \\
\hline 13 & 17.9 & -.1 & 10.1 & 17.7 & 2.2 & 9.9 & 17.8 & 3.8 & 11.1 \\
\hline 14 & 19.3 & 6.9 & 13.4 & 18.8 & 7.6 & 13.2 & 18.4 & 9.6 & 13.9 \\
\hline 15 & 16.3 & 7.1 & 12.0 & 15.6 & 7.1 & 11.8 & 15.9 & 7.3 & 11.5 \\
\hline 16 & 15.4 & 6.4 & 9.7 & 15.6 & 6.3 & 9.5 & 14.5 & 6.6 & 9.6 \\
\hline 17 & 18.6 & 9.5 & 13.1 & 17.9 & 9.6 & 12.8 & 18.2 & 10.3 & 13.0 \\
\hline 18 & 15.5 & 8.6 & 10.8 & 14.7 & 9.0 & 10.8 & 15.5 & 9.0 & 10.5 \\
\hline 19 & 17.3 & 6.3 & 11.9 & 17.7 & 7.3 & 11.9 & 17.6 & 7.9 & 12.6 \\
\hline 20 & 20.1 & 6.3 & 13.3 & 19.4 & 7.1 & 13.1 & 19.7 & 8.7 & 14.2 \\
\hline 21 & 12.9 & 7.5 & 9.9 & 12.8 & 7.5 & 9.6 & 12.5 & 8.5 & 10.1 \\
\hline 22 & 12.8 & 7.4 & 9.9 & 12.6 & 7.6 & 9.8 & 13.2 & 8.0 & 10.2 \\
\hline 23 & 11.1 & 7.5 & 9.1 & 10.8 & 7.6 & 8.8 & 11.4 & 7.8 & 9.2 \\
\hline 24 & 8.8 & 6.9 & 7.8 & 8.3 & 6.5 & 7.3 & 8.7 & 6.9 & 7.8 \\
\hline 25 & 10.2 & 6.6 & 8.1 & 9.5 & 6.0 & 7.4 & 9.9 & 6.6 & 7.9 \\
\hline 26 & 11.6 & 8.8 & 10.0 & 11.3 & 8.0 & 9.5 & 12.1 & 8.2 & 9.9 \\
\hline 27 & 18.7 & 10.7 & 13.9 & 18.1 & 10.6 & 13.6 & 18.1 & 11.0 & 14.0 \\
\hline 28 & 25.4 & 10.9 & 17.7 & 24.9 & 10.4 & 17.6 & 25.9 & 12.1 & 18.7 \\
\hline 29 & 22.8 & 12.9 & 15.9 & 21.7 & 12.4 & 15.4 & 22.0 & 13.4 & 15.9 \\
\hline 30 & 22.9 & 11.7 & 16.1 & 21.8 & 11.6 & 15.8 & 22.5 & 12.6 & 16.6 \\
\hline 31 & 14.2 & 11.1 & 12.8 & 14.1 & 10.8 & 12.4 & 14.5 & 11.1 & 12.7 \\
\hline Month & 26.5 & -.1 & 11.4 & 25.0 & 2.2 & 11.2 & 26.6 & 3.8 & 11.8 \\
\hline
\end{tabular}


Table 12. Daily, monthly, and annual statistics for air temperature for U.S. Geological Survey stations 01104430, Cambridge Reservoir near Kendal Green; 01104480, Stony Brook Reservoir in Waltham; and 422302071083801, Fresh Pond Reservoir at Cambridge, Massachusetts, for water year 2005.-Continued

[Units are in degrees Celsius. Max, maximum; Min, minimum; e, estimated]

\begin{tabular}{|c|c|c|c|c|c|c|c|c|c|}
\hline \multirow{2}{*}{$\begin{array}{l}\text { Month } \\
\text { and } \\
\text { date }\end{array}$} & \multicolumn{3}{|c|}{$\begin{array}{l}\text { Cambridge Reservoir } \\
\text { near Kendal Green } \\
\text { (01104430) }\end{array}$} & \multicolumn{3}{|c|}{$\begin{array}{c}\text { Stony Brook Reservoir at } \\
\text { dam near Waltham } \\
(01104480)\end{array}$} & \multicolumn{3}{|c|}{$\begin{array}{c}\text { Fresh Pond gate house } \\
\text { at Cambridge } \\
(422302071083801)\end{array}$} \\
\hline & Max & Min & Mean & Max & Min & Mean & Max & Min & Mean \\
\hline \multicolumn{10}{|c|}{ June } \\
\hline 1 & 18.8 & 9.2 & 13.7 & 18.5 & 9.2 & 13.6 & 17.8 & 10.3 & 13.9 \\
\hline 2 & 25.2 & 10.5 & 16.9 & 24.4 & 10.3 & 16.4 & 25.6 & 10.9 & 17.2 \\
\hline 3 & 25.4 & 10.6 & 18.1 & 24.9 & 10.6 & 18.0 & 26.1 & 11.2 & 18.7 \\
\hline 4 & 29.1 & 14.2 & 21.6 & 29.5 & 14.4 & 21.7 & 29.7 & 15.8 & 22.5 \\
\hline 5 & 31.2 & 15.6 & 23.7 & 30.7 & 15.9 & 23.5 & 31.0 & 16.9 & 24.5 \\
\hline 6 & 22.6 & 12.4 & 16.2 & 22.6 & 11.9 & 15.9 & 21.2 & 12.3 & 15.7 \\
\hline 7 & 29.8 & 17.3 & 23.4 & 30.3 & 18.0 & 24.1 & 31.1 & 19.4 & 25.0 \\
\hline 8 & 30.4 & 14.8 & 23.2 & 31.9 & 15.7 & 23.7 & 32.3 & 18.7 & 25.2 \\
\hline 9 & 26.1 & 17.5 & 21.8 & 25.7 & 17.8 & 21.7 & 25.3 & 18.9 & 21.8 \\
\hline 10 & 28.3 & 17.1 & 23.2 & 27.6 & 17.2 & 23.3 & 28.6 & 18.0 & 24.4 \\
\hline 11 & 30.8 & 20.8 & 25.4 & 31.4 & 20.7 & 25.6 & 32.5 & 21.3 & 26.5 \\
\hline 12 & 29.9 & 21.1 & 25.3 & 30.3 & 21.0 & 25.5 & 31.8 & 22.0 & 26.5 \\
\hline 13 & 31.4 & 22.5 & 26.3 & 31.9 & 22.7 & 26.7 & 31.8 & 23.8 & 27.6 \\
\hline 14 & 26.0 & 12.6 & 19.6 & 25.7 & 12.7 & 20.0 & 26.5 & 12.5 & 20.1 \\
\hline 15 & 12.9 & 9.9 & 10.9 & 12.8 & 9.4 & 10.4 & 13.4 & 9.9 & 10.9 \\
\hline 16 & 16.7 & 9.7 & 12.6 & 16.4 & 9.7 & 12.2 & 15.9 & 10.0 & 12.4 \\
\hline 17 & 22.4 & 10.9 & 15.0 & 22.1 & 10.8 & 14.5 & 22.8 & 11.0 & 14.5 \\
\hline 18 & 19.9 & 13.1 & 15.3 & 19.9 & 12.5 & 14.8 & 19.0 & 12.5 & 14.9 \\
\hline 19 & 17.2 & 10.0 & 14.3 & 18.4 & 10.1 & 14.1 & 17.8 & 11.9 & 14.6 \\
\hline 20 & 25.0 & 8.2 & 17.3 & 26.0 & 9.1 & 17.1 & 25.5 & 9.8 & 17.9 \\
\hline 21 & 27.8 & 12.1 & 20.3 & 27.8 & 11.5 & 20.7 & 28.7 & 13.2 & 21.7 \\
\hline 22 & 22.0 & 16.4 & 19.0 & 22.4 & 15.9 & 19.2 & 23.3 & 17.4 & 20.0 \\
\hline 23 & 23.6 & 9.6 & 18.1 & 24.4 & 11.1 & 18.0 & 24.7 & 12.5 & 19.0 \\
\hline 24 & 28.7 & 12.5 & 21.7 & 29.0 & 13.3 & 22.1 & 29.9 & 15.5 & 23.1 \\
\hline 25 & 32.9 & 17.9 & 25.2 & 32.6 & 18.6 & 26.0 & 34.0 & 19.5 & 27.0 \\
\hline 26 & 33.6 & 20.2 & 26.6 & 34.1 & 20.3 & 26.7 & 35.4 & 22.3 & 27.9 \\
\hline 27 & 31.3 & 19.6 & 25.2 & 30.8 & 20.5 & 25.3 & 29.5 & 22.4 & 25.4 \\
\hline 28 & 26.4 & 22.5 & 23.9 & 26.4 & 22.5 & 23.9 & 28.4 & 23.8 & 25.1 \\
\hline 29 & 29.0 & 21.2 & 23.9 & 29.0 & 21.0 & 23.9 & 29.6 & 22.5 & 25.0 \\
\hline 30 & 27.6 & 18.5 & 23.0 & 26.1 & 18.5 & 22.5 & 26.9 & 18.4 & 23.4 \\
\hline Month & 33.6 & 8.2 & 20.4 & 34.1 & 9.1 & 20.4 & 35.4 & 9.8 & 21.1 \\
\hline
\end{tabular}


Table 12. Daily, monthly, and annual statistics for air temperature for U.S. Geological Survey stations 01104430, Cambridge Reservoir near Kendal Green; 01104480, Stony Brook Reservoir in Waltham; and 422302071083801, Fresh Pond Reservoir at Cambridge, Massachusetts, for water year 2005.-Continued

[Units are in degrees Celsius. Max, maximum; Min, minimum; e, estimated]

\begin{tabular}{|c|c|c|c|c|c|c|c|c|c|}
\hline \multirow{2}{*}{$\begin{array}{l}\text { Month } \\
\text { and } \\
\text { date }\end{array}$} & \multicolumn{3}{|c|}{$\begin{array}{c}\text { Cambridge Reservoir } \\
\text { near Kendal Green } \\
\text { (01104430) }\end{array}$} & \multicolumn{3}{|c|}{$\begin{array}{c}\text { Stony Brook Reservoir at } \\
\text { dam near Waltham } \\
(01104480)\end{array}$} & \multicolumn{3}{|c|}{$\begin{array}{c}\text { Fresh Pond gate house } \\
\text { at Cambridge } \\
(422302071083801)\end{array}$} \\
\hline & Max & Min & Mean & Max & Min & Mean & Max & Min & Mean \\
\hline \multicolumn{10}{|c|}{ July } \\
\hline 1 & 24.9 & 16.9 & 20.3 & 24.8 & 16.6 & 20.0 & 24.6 & 16.9 & 20.2 \\
\hline 2 & 26.6 & 17.7 & 22.9 & 25.4 & 17.8 & 22.6 & 26.2 & 20.7 & 23.8 \\
\hline 3 & 25.7 & 12.4 & 20.1 & 25.6 & 13.0 & 20.1 & 25.9 & 14.8 & 21.2 \\
\hline 4 & 27.5 & 12.3 & 19.7 & 27.0 & 13.0 & 20.4 & 26.3 & 15.0 & 21.1 \\
\hline 5 & 29.4 & 13.1 & 22.2 & 29.1 & 13.8 & 22.5 & 30.6 & 16.0 & 23.7 \\
\hline 6 & 22.8 & 16.2 & 19.8 & 22.7 & 15.6 & 19.5 & 23.3 & 16.0 & 20.2 \\
\hline 7 & 18.5 & 15.4 & 16.9 & 18.6 & 15.0 & 16.4 & 18.4 & 15.9 & 17.0 \\
\hline 8 & 16.7 & 13.5 & 15.5 & 16.4 & 12.9 & 14.9 & 17.0 & 13.4 & 15.4 \\
\hline 9 & 24.4 & 13.0 & 17.6 & 24.3 & 12.9 & 17.3 & 25.7 & 13.4 & 18.1 \\
\hline 10 & 30.5 & 16.4 & 23.0 & 30.0 & 17.0 & 23.2 & 31.2 & 17.8 & 24.3 \\
\hline 11 & 31.7 & 20.1 & 26.4 & 30.4 & 21.2 & 26.1 & 31.7 & 21.6 & 27.5 \\
\hline 12 & 24.9 & 17.2 & 21.2 & 24.0 & 17.1 & 20.8 & 23.6 & 18.0 & 21.1 \\
\hline 13 & 25.5 & 16.0 & 21.0 & 25.3 & 15.9 & 20.7 & 26.5 & 17.1 & 21.9 \\
\hline 14 & 28.2 & 18.6 & 23.4 & 28.4 & 18.6 & 23.4 & 29.8 & 19.8 & 24.6 \\
\hline 15 & 28.6 & 19.6 & 24.0 & 28.0 & 19.9 & 23.8 & 28.6 & 22.1 & 24.7 \\
\hline 16 & 28.5 & 18.4 & 23.3 & 29.0 & 18.7 & 23.4 & 31.4 & 20.3 & 25.3 \\
\hline 17 & 27.5 & 20.2 & 23.3 & 27.3 & 20.7 & 23.7 & 30.2 & 21.7 & 25.1 \\
\hline 18 & 29.0 & 21.9 & 24.6 & 29.5 & 23.1 & 25.2 & 30.7 & 24.2 & 26.3 \\
\hline 19 & 31.6 & 21.7 & 25.8 & 32.2 & 23.2 & 26.6 & 33.3 & 24.5 & 28.0 \\
\hline 20 & 30.9 & 20.1 & 25.6 & 30.2 & 21.5 & 25.9 & 31.2 & 23.0 & 27.5 \\
\hline 21 & 29.1 & 18.3 & 23.8 & 29.2 & 19.1 & 24.3 & 30.5 & 22.0 & 26.0 \\
\hline 22 & 31.3 & 18.3 & 25.0 & 31.9 & 18.8 & 25.4 & 32.5 & 20.9 & 26.9 \\
\hline 23 & 27.1 & 17.7 & 22.8 & 26.5 & 19.0 & 22.6 & 27.2 & 20.4 & 23.7 \\
\hline 24 & 26.8 & 14.9 & 20.9 & 28.0 & 16.1 & 21.5 & 28.1 & 18.3 & 22.7 \\
\hline 25 & 27.2 & 17.4 & 22.9 & 28.8 & 19.2 & 23.9 & 29.3 & 21.0 & 25.1 \\
\hline 26 & 31.4 & 18.4 & 25.0 & 33.0 & 19.1 & 26.5 & 34.0 & 21.3 & 27.9 \\
\hline 27 & 33.3 & 20.6 & 27.0 & 33.4 & 20.8 & 27.6 & 35.2 & 21.8 & 28.9 \\
\hline 28 & 23.9 & 14.5 & 20.4 & 23.6 & 15.6 & 20.6 & 23.6 & 18.0 & 21.2 \\
\hline 29 & 26.6 & 12.7 & 20.2 & 27.7 & 13.5 & 21.3 & 28.1 & 15.7 & 22.5 \\
\hline 30 & 26.6 & 17.5 & 21.4 & 26.4 & 18.5 & 21.8 & 26.2 & 19.7 & 22.4 \\
\hline 31 & 21.5 & 16.6 & 19.4 & 21.6 & 17.3 & 19.5 & 21.7 & 19.1 & 20.3 \\
\hline Month & 33.3 & 12.3 & 22.1 & 33.4 & 12.9 & 22.3 & 35.2 & 13.4 & 23.4 \\
\hline
\end{tabular}


Table 12. Daily, monthly, and annual statistics for air temperature for U.S. Geological Survey stations 01104430, Cambridge Reservoir near Kendal Green; 01104480, Stony Brook Reservoir in Waltham; and 422302071083801, Fresh Pond Reservoir at Cambridge, Massachusetts, for water year 2005.-Continued

[Units are in degrees Celsius. Max, maximum; Min, minimum; e, estimated]

\begin{tabular}{|c|c|c|c|c|c|c|c|c|c|}
\hline \multirow{2}{*}{$\begin{array}{l}\text { Month } \\
\text { and } \\
\text { date }\end{array}$} & \multicolumn{3}{|c|}{$\begin{array}{c}\text { Cambridge Reservoir } \\
\text { near Kendal Green } \\
\text { (01104430) }\end{array}$} & \multicolumn{3}{|c|}{$\begin{array}{c}\text { Stony Brook Reservoir at } \\
\text { dam near Waltham } \\
(01104480)\end{array}$} & \multicolumn{3}{|c|}{$\begin{array}{c}\text { Fresh Pond gate house } \\
\text { at Cambridge } \\
\text { (422302071083801) }\end{array}$} \\
\hline & Max & Min & Mean & Max & Min & Mean & Max & Min & Mean \\
\hline \multicolumn{10}{|c|}{ August } \\
\hline 1 & 27.2 & 18.0 & 21.5 & 27.7 & 18.2 & 22.2 & 29.1 & 18.9 & 23.1 \\
\hline 2 & 29.5 & 18.7 & 23.7 & 29.8 & 18.2 & 23.9 & 30.3 & 19.0 & 25.0 \\
\hline 3 & 30.1 & 18.7 & 24.3 & 29.6 & 20.6 & 24.8 & 29.2 & 21.9 & 25.4 \\
\hline 4 & 28.9 & 18.9 & 24.0 & 28.9 & 19.0 & 23.9 & 28.1 & 20.7 & 24.4 \\
\hline 5 & 32.3 & 21.1 & 25.3 & 32.7 & 21.0 & 25.1 & 34.5 & 22.2 & 26.2 \\
\hline 6 & 27.6 & 17.3 & 23.0 & 28.5 & 18.3 & 22.8 & 27.6 & 20.5 & 24.0 \\
\hline 7 & 28.4 & 16.1 & 22.3 & 29.0 & 16.2 & 23.0 & 30.2 & 18.1 & 24.2 \\
\hline 8 & 31.6 & 18.1 & 24.7 & 32.0 & 18.5 & 24.7 & 32.8 & 19.9 & 26.0 \\
\hline 9 & 29.0 & 21.1 & 24.4 & 29.1 & 21.3 & 24.7 & 30.7 & 22.8 & 25.9 \\
\hline 10 & 30.6 & 20.1 & 24.8 & 30.6 & 20.5 & 25.2 & 32.3 & 22.0 & 26.4 \\
\hline 11 & 31.0 & 20.6 & 25.5 & 30.7 & 20.5 & 25.3 & 31.7 & 22.1 & 26.0 \\
\hline 12 & 29.2 & 21.4 & 24.7 & 28.7 & 21.4 & 24.9 & 28.2 & 22.1 & 25.2 \\
\hline 13 & 32.6 & 22.8 & 27.3 & 32.8 & 23.7 & 27.8 & 33.7 & 25.2 & 28.9 \\
\hline 14 & 32.0 & 19.1 & 24.5 & 31.2 & 19.3 & 24.3 & 31.9 & 19.9 & 25.3 \\
\hline 15 & 20.4 & 16.0 & 19.1 & 20.2 & 16.3 & 18.7 & 20.9 & 17.7 & 19.4 \\
\hline 16 & 24.5 & 14.2 & 19.7 & 24.7 & 14.0 & 19.5 & 24.2 & 15.4 & 20.0 \\
\hline 17 & 28.4 & 17.7 & 22.7 & 27.0 & 17.2 & 22.3 & 27.8 & 19.0 & 23.6 \\
\hline 18 & 24.2 & 15.1 & 19.8 & 24.7 & 15.2 & 19.8 & 25.9 & 16.4 & 21.1 \\
\hline 19 & 25.3 & 14.3 & 19.8 & 24.9 & 14.6 & 19.9 & 24.4 & 16.5 & 20.7 \\
\hline 20 & 26.1 & 18.9 & 22.2 & 25.9 & 18.6 & 22.1 & 25.8 & 19.3 & 22.1 \\
\hline 21 & 29.7 & 20.2 & 23.9 & 29.7 & 20.9 & 24.5 & 31.7 & 21.8 & 25.7 \\
\hline 22 & 28.2 & 18.0 & 22.5 & 27.7 & 18.0 & 23.1 & 28.2 & 20.6 & 24.4 \\
\hline 23 & 25.6 & 14.9 & 19.7 & 24.7 & 15.6 & 19.9 & 25.4 & 17.2 & 21.2 \\
\hline 24 & 24.0 & 15.6 & 19.3 & 22.8 & 16.0 & 19.1 & 24.1 & 17.3 & 20.3 \\
\hline 25 & 25.3 & 14.4 & 19.3 & 25.3 & 14.2 & 19.2 & 25.9 & 15.7 & 20.1 \\
\hline 26 & 27.3 & 13.1 & 19.8 & 28.9 & 13.9 & 20.7 & 29.9 & 16.0 & 22.1 \\
\hline 27 & 27.5 & 14.0 & 20.9 & 27.8 & 14.4 & 21.4 & 29.2 & 17.0 & 23.0 \\
\hline 28 & 28.1 & 17.5 & 22.2 & 28.0 & 17.1 & 22.8 & 29.9 & 19.5 & 24.5 \\
\hline 29 & 28.9 & 21.9 & 24.0 & 29.3 & 21.4 & 23.9 & 30.8 & 21.9 & 25.0 \\
\hline 30 & 23.1 & 21.6 & 22.2 & 22.9 & 21.1 & 21.8 & 23.5 & 21.9 & 22.7 \\
\hline 31 & 27.3 & 22.2 & 24.6 & 26.9 & 21.9 & 24.3 & 28.2 & 23.0 & 25.6 \\
\hline Month & 32.6 & 13.1 & 22.6 & 32.8 & 13.9 & 22.8 & 34.5 & 15.4 & 23.8 \\
\hline
\end{tabular}


Table 12. Daily, monthly, and annual statistics for air temperature for U.S. Geological Survey stations 01104430, Cambridge Reservoir near Kendal Green; 01104480, Stony Brook Reservoir in Waltham; and 422302071083801, Fresh Pond Reservoir at Cambridge, Massachusetts, for water year 2005.-Continued

[Units are in degrees Celsius. Max, maximum; Min, minimum; e, estimated]

\begin{tabular}{|c|c|c|c|c|c|c|c|c|c|}
\hline \multirow{2}{*}{$\begin{array}{l}\text { Month } \\
\text { and } \\
\text { date }\end{array}$} & \multicolumn{3}{|c|}{$\begin{array}{c}\text { Cambridge Reservoir } \\
\text { near Kendal Green } \\
\text { (01104430) }\end{array}$} & \multicolumn{3}{|c|}{$\begin{array}{c}\text { Stony Brook Reservoir at } \\
\text { dam near Waltham } \\
(01104480)\end{array}$} & \multicolumn{3}{|c|}{$\begin{array}{c}\text { Fresh Pond gate house } \\
\text { at Cambridge } \\
\text { (422302071083801) }\end{array}$} \\
\hline & Max & Min & Mean & Max & Min & Mean & Max & Min & Mean \\
\hline \multicolumn{10}{|c|}{ September } \\
\hline 1 & 27.8 & 18.3 & 22.7 & 27.1 & 19.2 & 23.0 & 27.9 & 20.4 & 24.0 \\
\hline 2 & 28.0 & 15.9 & 21.7 & 28.4 & 15.9 & 22.3 & 28.9 & 17.7 & 23.7 \\
\hline 3 & 26.2 & 15.9 & 21.0 & 25.7 & 17.2 & 21.3 & 26.5 & 19.1 & 22.6 \\
\hline 4 & 25.9 & 15.0 & 19.4 & 24.6 & 14.8 & 19.3 & 24.7 & 17.4 & 20.6 \\
\hline 5 & 23.1 & 12.1 & 17.7 & 23.0 & 12.5 & 17.7 & 23.2 & 15.2 & 18.9 \\
\hline 6 & 25.0 & 11.0 & 17.8 & 24.6 & 12.0 & 18.0 & 25.2 & 12.6 & 19.0 \\
\hline 7 & 27.4 & 12.3 & 19.7 & 28.8 & 12.5 & 20.2 & 29.7 & 14.6 & 21.4 \\
\hline 8 & 28.0 & 13.8 & 21.1 & 28.9 & 14.1 & 21.5 & 30.1 & 15.9 & 22.8 \\
\hline 9 & 23.7 & 15.0 & 19.6 & 23.4 & 16.1 & 19.8 & 23.5 & 17.9 & 20.7 \\
\hline 10 & 21.4 & 11.0 & 16.3 & 21.6 & 11.4 & 16.4 & 22.0 & 13.5 & 17.6 \\
\hline 11 & 22.5 & 8.4 & 16.1 & 23.8 & 9.2 & 16.2 & 24.8 & 11.3 & 17.8 \\
\hline 12 & 30.7 & 16.0 & 22.8 & 30.7 & 16.1 & 23.4 & 31.4 & 17.3 & 24.2 \\
\hline 13 & 29.0 & 18.4 & 22.9 & 27.8 & 18.6 & 22.9 & 26.9 & 19.8 & 23.2 \\
\hline 14 & 29.4 & 19.3 & 23.7 & 30.4 & 19.2 & 23.8 & 31.2 & 20.0 & 24.7 \\
\hline 15 & 23.6 & 21.0 & 22.1 & 23.7 & 20.0 & 21.9 & 24.6 & 20.7 & 22.8 \\
\hline 16 & 21.4 & 18.0 & 19.9 & 21.0 & 17.6 & 19.3 & 22.0 & 18.1 & 20.0 \\
\hline 17 & 21.3 & 17.2 & 18.8 & 20.8 & 16.8 & 18.3 & 21.0 & 17.4 & 18.9 \\
\hline 18 & 23.6 & 15.2 & 19.7 & 23.3 & 15.6 & 19.5 & 23.6 & 17.2 & 20.5 \\
\hline 19 & 26.1 & 13.7 & 19.0 & 26.8 & 13.4 & 19.5 & 28.0 & 14.9 & 21.1 \\
\hline 20 & 24.1 & 15.2 & 20.0 & 24.0 & 15.3 & 20.0 & 24.8 & 17.6 & 21.5 \\
\hline 21 & 24.9 & 13.3 & 19.2 & 25.4 & 15.6 & 19.9 & 26.5 & 17.2 & 21.2 \\
\hline 22 & 27.8 & 13.3 & 20.8 & 28.2 & 13.5 & 21.3 & 29.4 & 15.3 & 22.5 \\
\hline 23 & 25.9 & 15.4 & 20.6 & 24.9 & 15.8 & 20.6 & 26.3 & 16.7 & 21.5 \\
\hline 24 & 19.0 & 8.2 & 13.4 & 19.2 & 8.4 & 13.9 & 19.1 & 10.7 & 15.2 \\
\hline 25 & 18.3 & 6.4 & 13.4 & 19.0 & 6.0 & 13.8 & 19.5 & 7.6 & 15.1 \\
\hline 26 & 22.5 & 16.5 & 19.9 & 22.6 & 16.4 & 20.0 & 23.6 & 17.5 & 20.8 \\
\hline 27 & 20.8 & 9.3 & 17.8 & 20.6 & 11.3 & 17.9 & 21.6 & 13.2 & 19.0 \\
\hline 28 & 20.9 & 6.9 & 14.0 & 22.2 & 7.8 & 14.7 & 22.2 & 9.2 & 15.8 \\
\hline 29 & 23.2 & 8.7 & 15.2 & 23.2 & 9.7 & 15.2 & 24.7 & 11.2 & 17.0 \\
\hline 30 & 15.9 & 6.2 & 10.3 & 17.0 & 7.1 & 10.9 & 18.0 & 8.6 & 12.5 \\
\hline Month & 30.7 & 6.2 & 18.9 & 30.7 & 6.0 & 19.1 & 31.4 & 7.6 & 20.2 \\
\hline Year & 33.6 & -21.0 & 9.8 & 34.1 & -20.4 & 10.0 & 35.4 & -19.1 & 10.8 \\
\hline Remarks & & Good & & & Good & & & Good & \\
\hline
\end{tabular}




\begin{tabular}{|c|c|c|c|c|c|c|c|c|c|}
\hline$\stackrel{\infty}{ \pm}$ & & 离 & 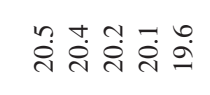 & 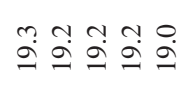 & 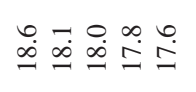 & 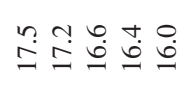 & 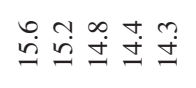 & 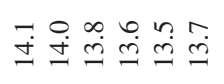 & \\
\hline & & E & 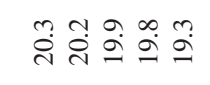 & 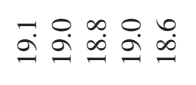 & 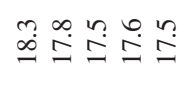 & 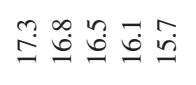 & 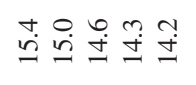 & 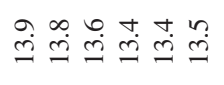 & \\
\hline & & 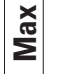 & 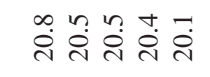 & 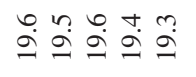 & 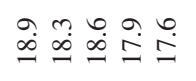 & 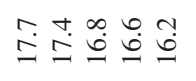 & $\begin{array}{l}\infty \\
\dot{n} \\
\dot{n}\end{array}$ & 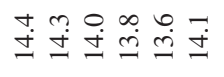 & \\
\hline & & 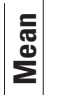 & 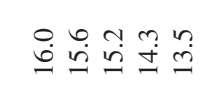 & 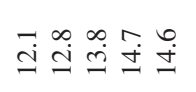 & 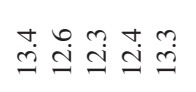 & 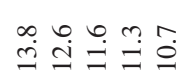 & 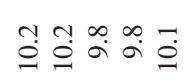 & 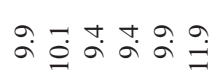 & \\
\hline & & $\mathbf{z}$ & 姜兰导宔 & 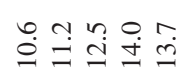 & 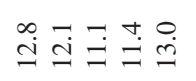 & $\stackrel{m}{=} \stackrel{\infty}{=} \stackrel{\infty}{0} \stackrel{?}{0} \stackrel{0}{0}$ & 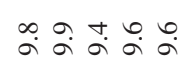 & 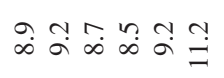 & " \\
\hline & & $\begin{array}{l}x \\
\tilde{n} \\
\tilde{\Sigma}\end{array}$ & 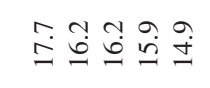 & 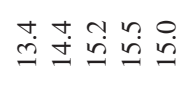 & 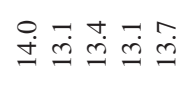 & 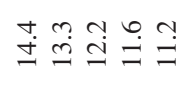 & 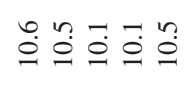 & $\stackrel{\infty}{\ominus} \exists \stackrel{n}{\Xi} \stackrel{n}{0} \stackrel{\sim}{=}$ & \\
\hline & & 离 & 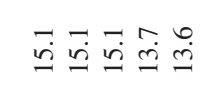 & 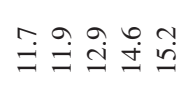 & 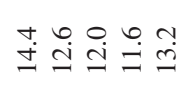 & 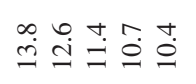 & 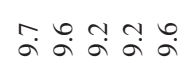 & $\stackrel{\circ}{\circ} \vec{\sigma} a_{\infty}+\dot{\infty} \dot{\infty} \stackrel{+}{=}$ & \\
\hline & & $\underline{\Sigma}$ & 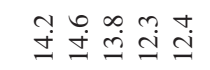 & 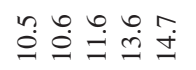 & 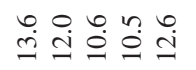 & 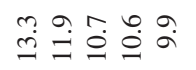 & స̆ & 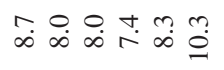 & \\
\hline & & $\mid \begin{array}{l}x \\
\Sigma \\
\Sigma\end{array}$ & 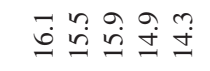 & 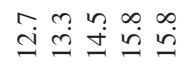 & 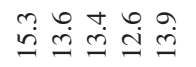 & $\stackrel{+}{\dot{I}} \stackrel{m}{=} \overrightarrow{\mathrm{I}} \stackrel{\circ}{=} \stackrel{\infty}{\dot{O}}$ & 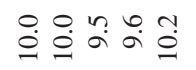 & 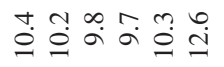 & \\
\hline & & $\mid$ & 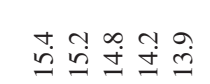 & 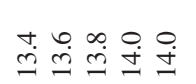 & 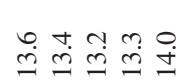 & 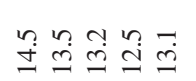 & 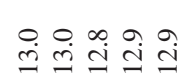 & 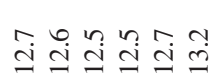 & \\
\hline & & E & 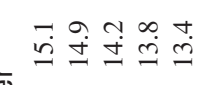 & 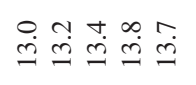 & 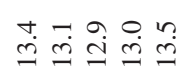 & 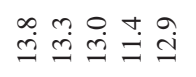 & 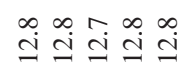 & 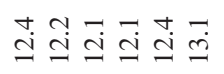 & \\
\hline & & 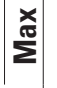 & 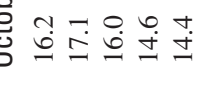 & 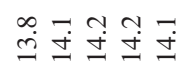 & 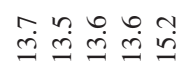 & 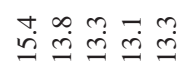 & 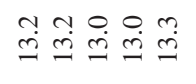 & 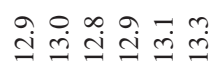 & \\
\hline 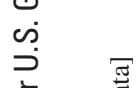 & & 离 & 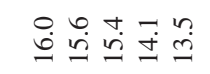 & 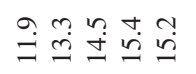 & 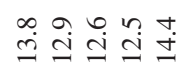 & $\stackrel{m}{\stackrel{9}{ \pm}} \stackrel{\circ}{\mathrm{I}} \stackrel{\circ}{=} \stackrel{\circ}{=} \stackrel{m}{=}$ & 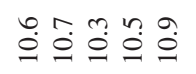 & 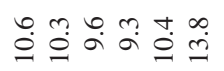 & \\
\hline 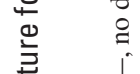 & & E & 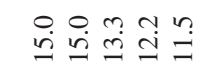 & 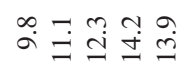 & 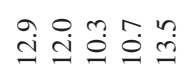 & 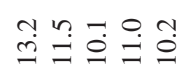 & ลे 동 & 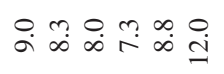 & \\
\hline $\begin{array}{l}\ddot{\mathrm{g}} \\
\ddot{\tilde{g}}\end{array}$ & & $\begin{array}{l}x \\
\text { a } \\
\Sigma\end{array}$ & 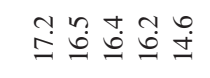 & 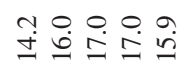 & 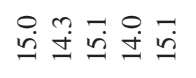 & 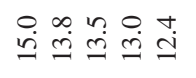 & 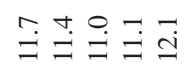 & $\stackrel{M}{\underline{I}} \hat{I} \stackrel{m}{=} \stackrel{\infty}{=} \stackrel{\infty}{=} \stackrel{0}{\underline{I}}$ & \\
\hline i & & $\begin{array}{l}\overline{\tilde{\varpi}} \\
\stackrel{\tilde{\Xi}}{\Sigma}\end{array}$ & 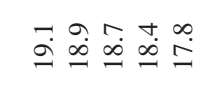 & $\stackrel{m}{=} \underset{I}{I} \stackrel{?}{=} \stackrel{?}{=}$ & 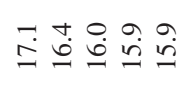 & 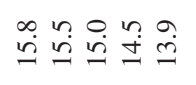 & 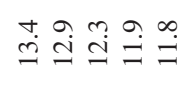 & 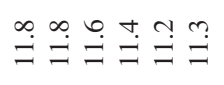 & \\
\hline $\begin{array}{l}\text { 慁 } \\
\dot{g}\end{array}$ & 흘 & $\bar{\Sigma}$ & 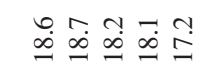 & فे & 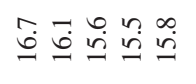 & 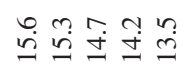 & 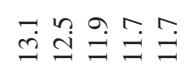 & 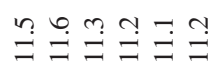 & \\
\hline 蒫 & & 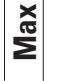 & 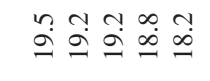 & 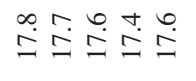 & 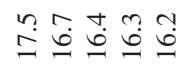 & 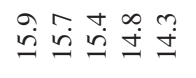 & 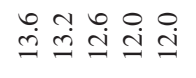 & 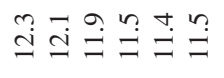 & \\
\hline 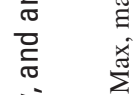 & : & 离 & 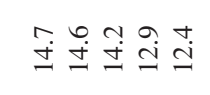 & 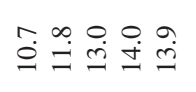 & $\widehat{i} \stackrel{\infty}{=} \stackrel{n}{=} \stackrel{n}{=} \stackrel{\infty}{=}$ & 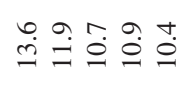 & $\stackrel{\infty}{\circ} \stackrel{0}{\circ} \stackrel{\circ}{\circ} \stackrel{0}{0}$ & 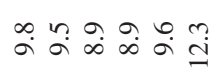 & \\
\hline$\frac{\stackrel{n}{0}}{0}$ & 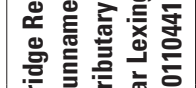 & $\dot{\Sigma}$ & 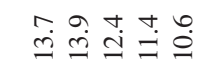 & 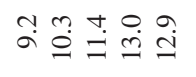 & 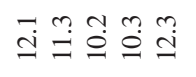 & 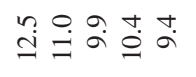 & $\tilde{\sigma} \tilde{\sigma} \sigma \dot{a} \sigma \hat{\sigma}$ & 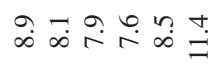 & \\
\hline 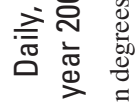 & & $\begin{array}{l}x \\
\tilde{\Sigma} \\
\tilde{\Sigma}\end{array}$ & 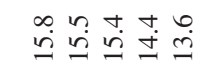 & 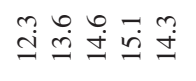 & 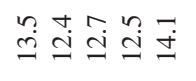 & 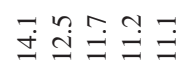 & 苍苍官 苍 & 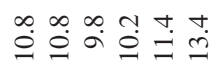 & \\
\hline 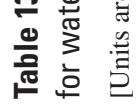 & 吾言 & & & & $=\simeq \cong$ & $\underline{-}=\infty 2$ & 고 & & \\
\hline
\end{tabular}




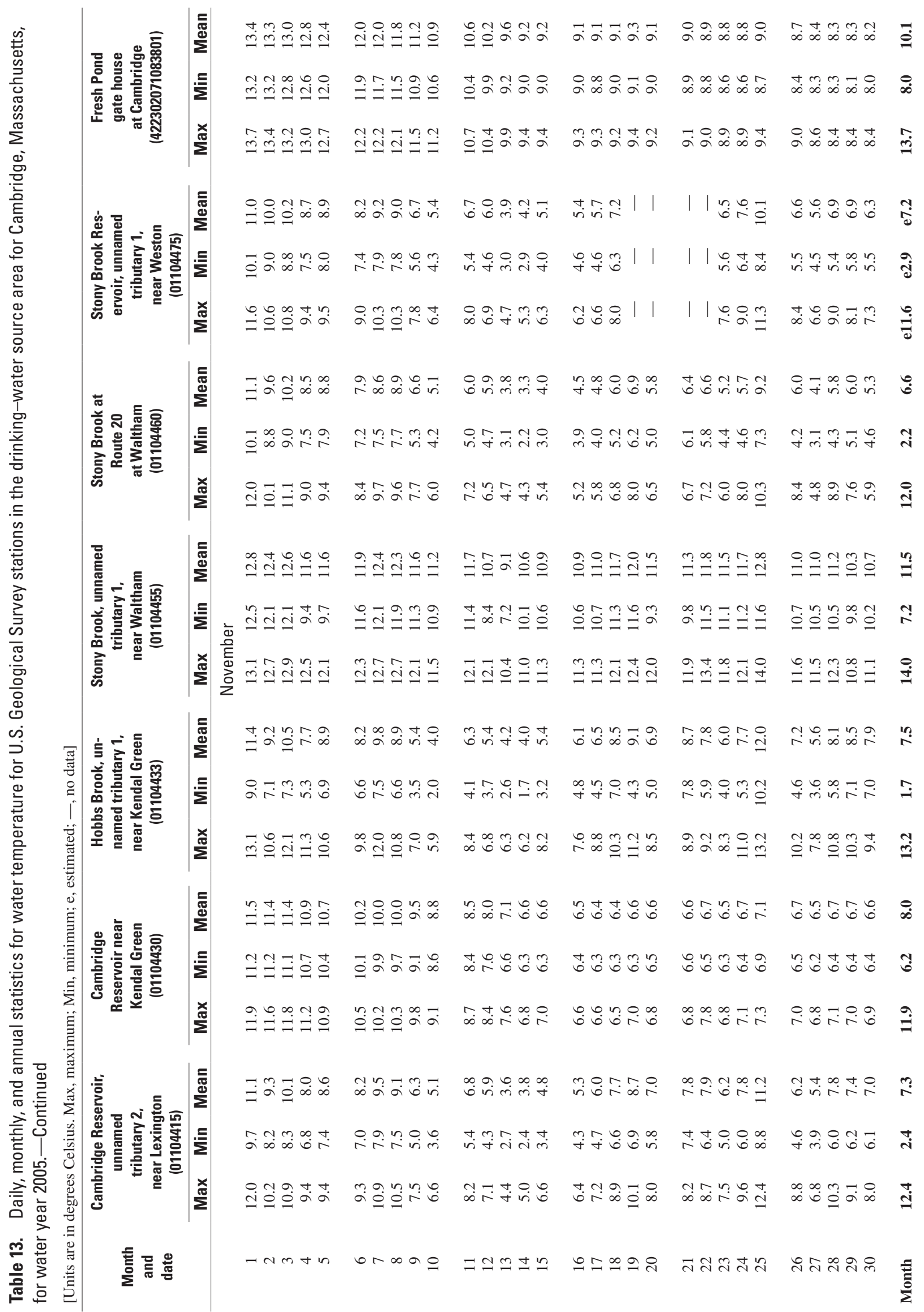




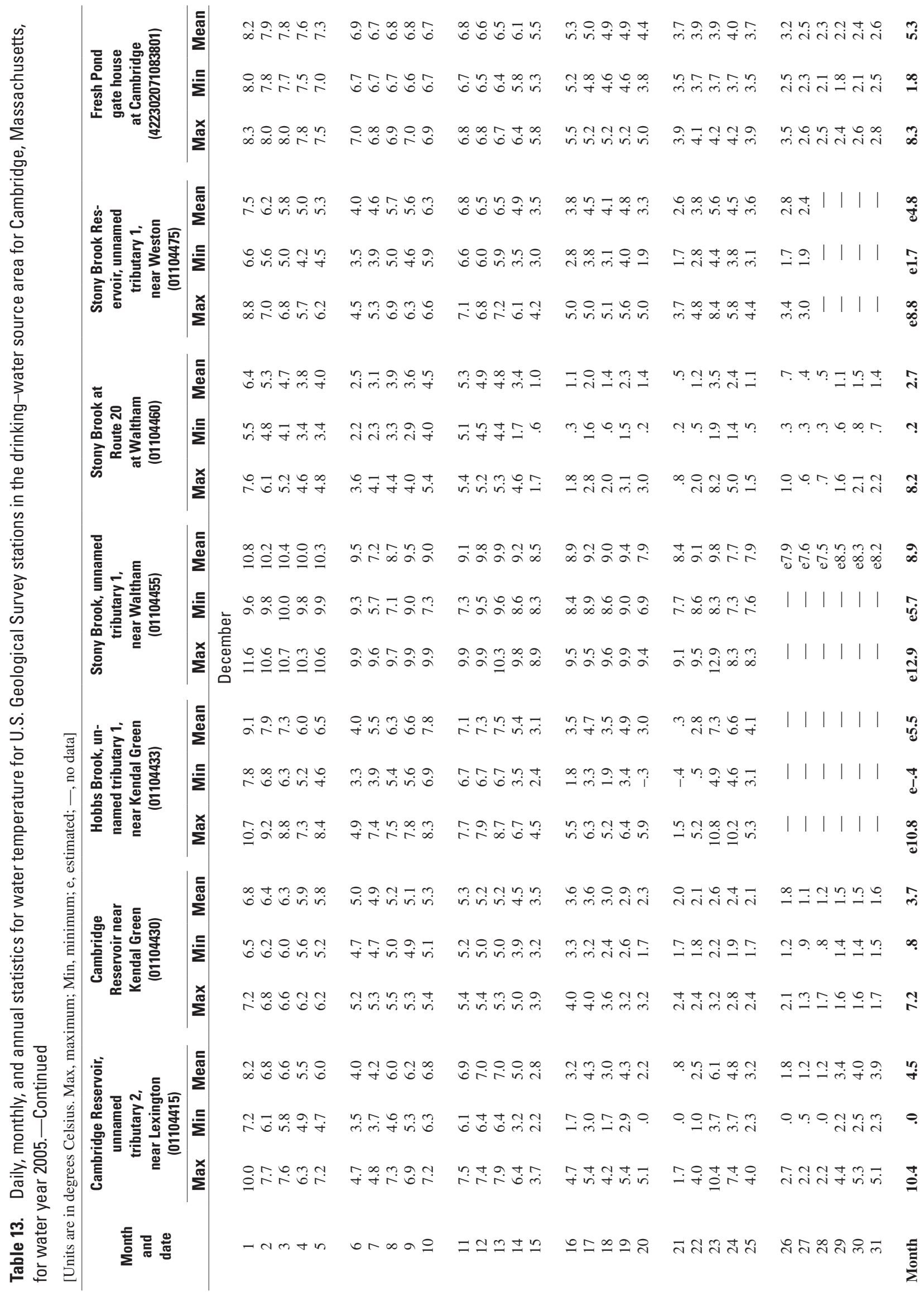




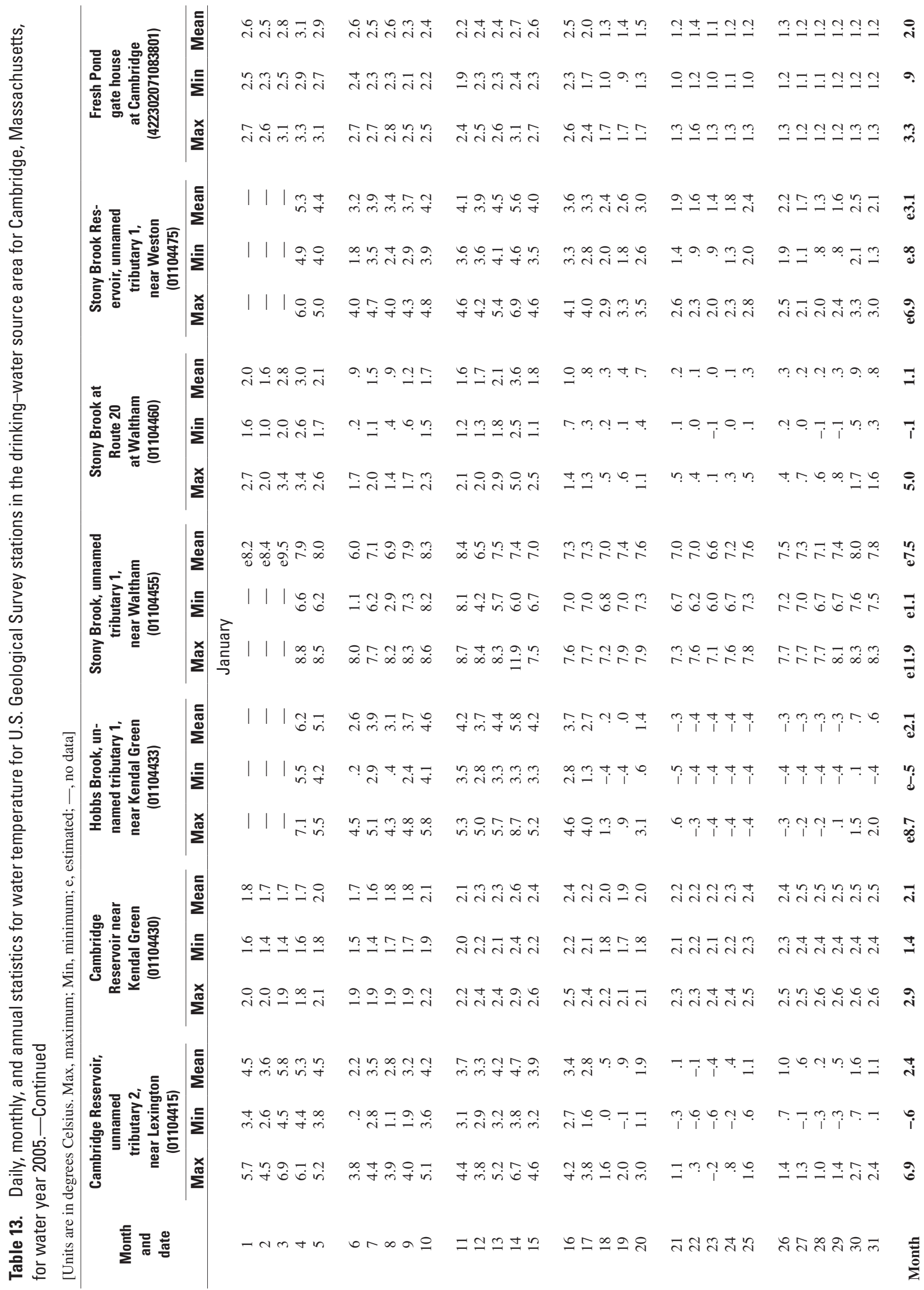




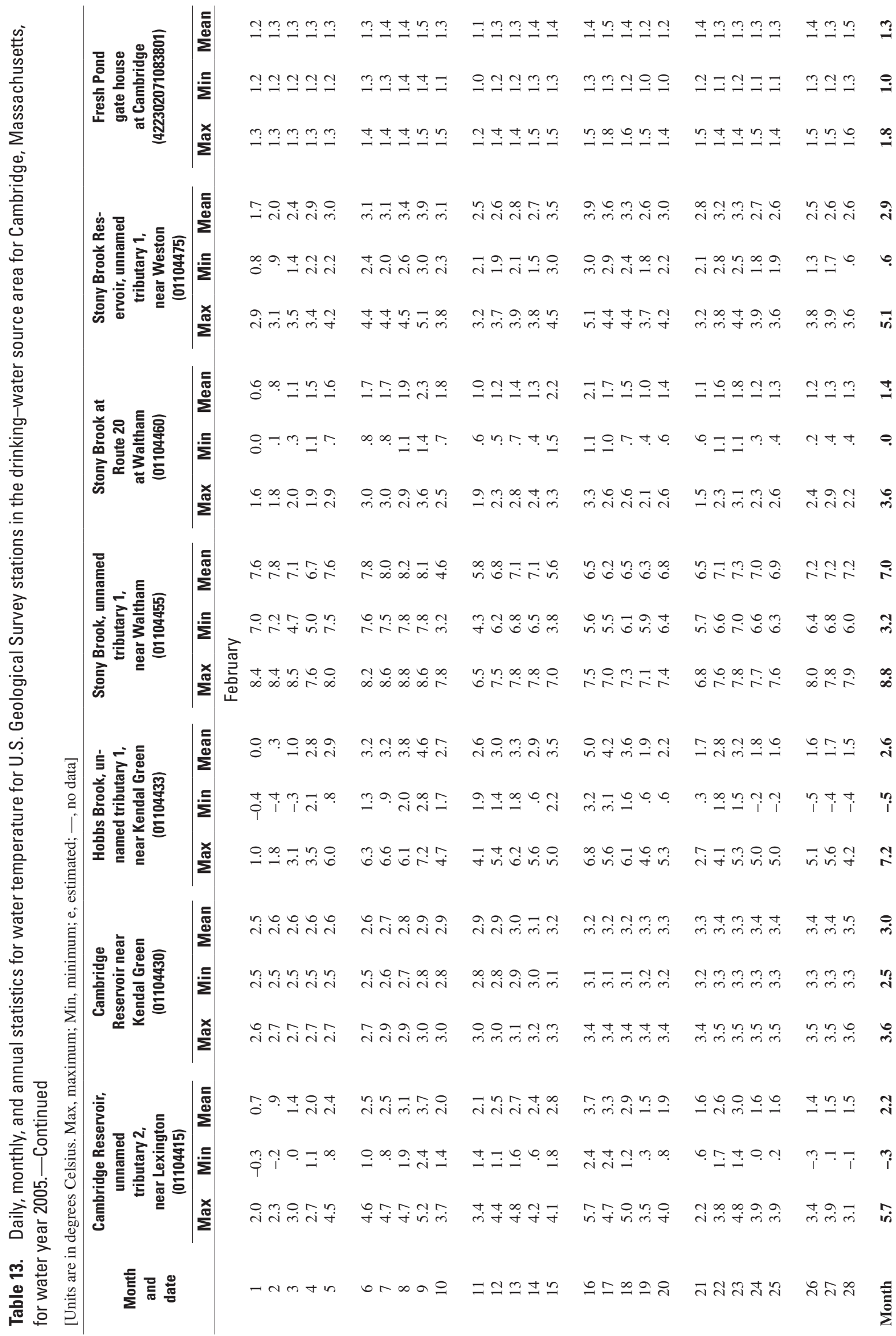




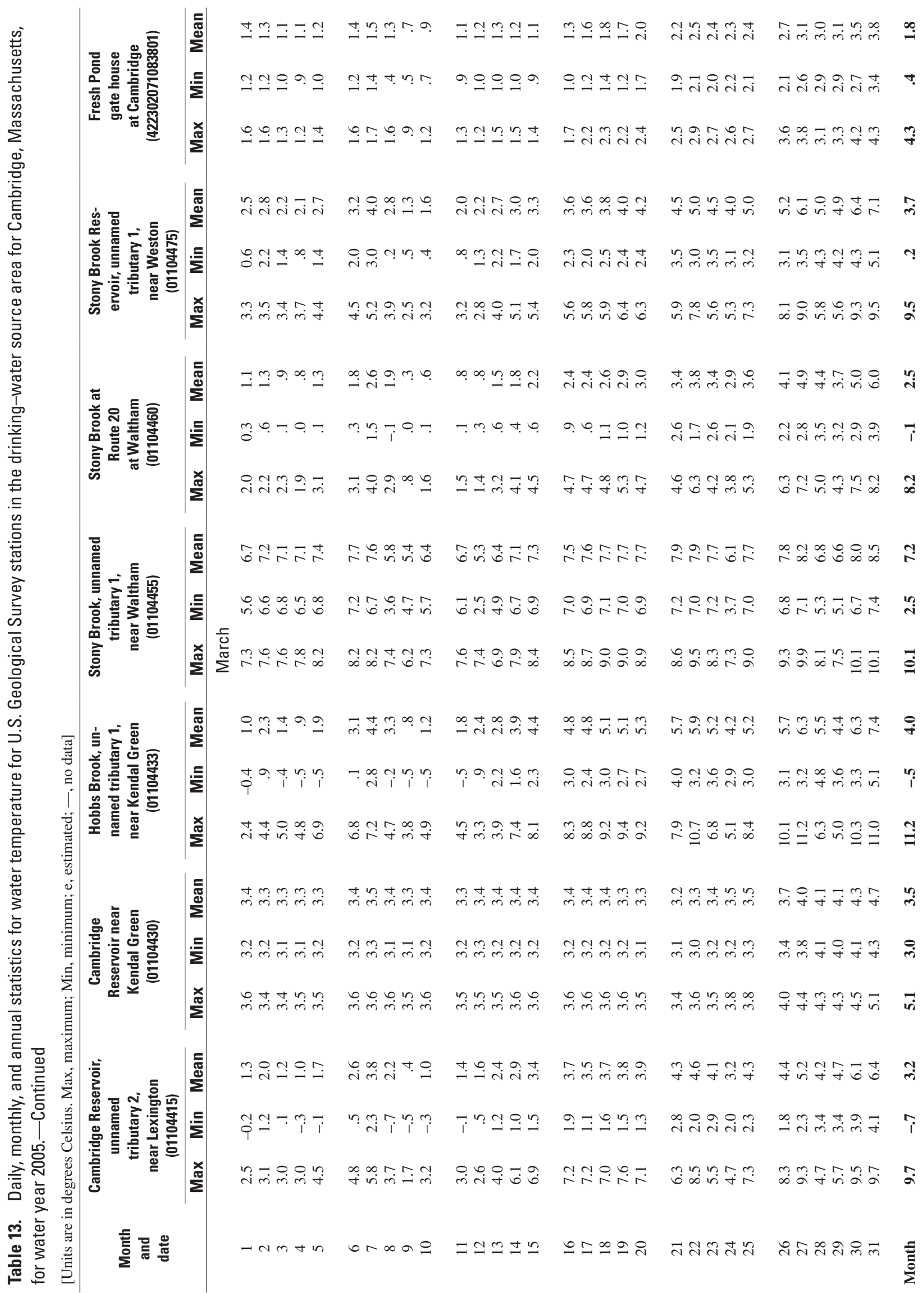




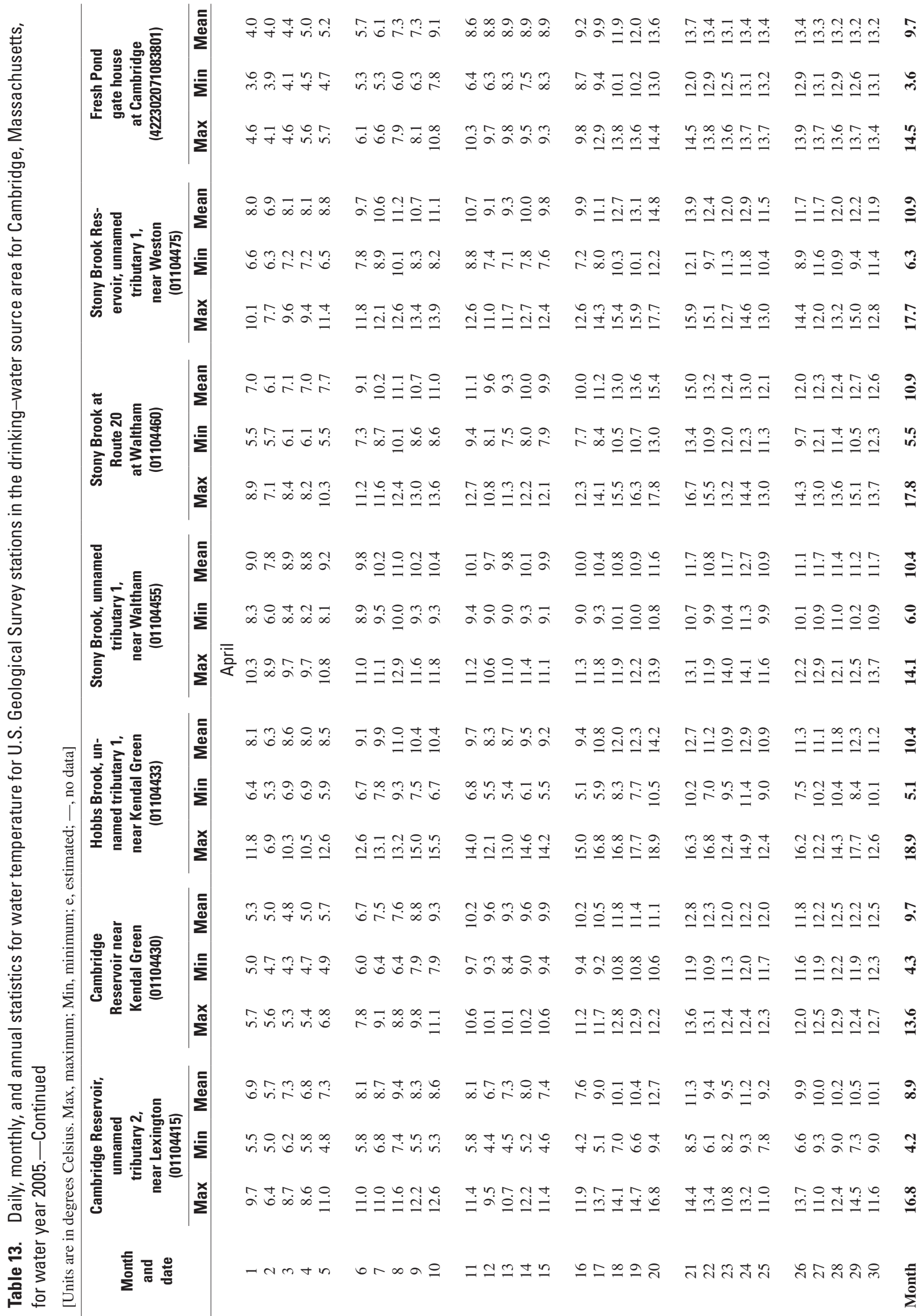




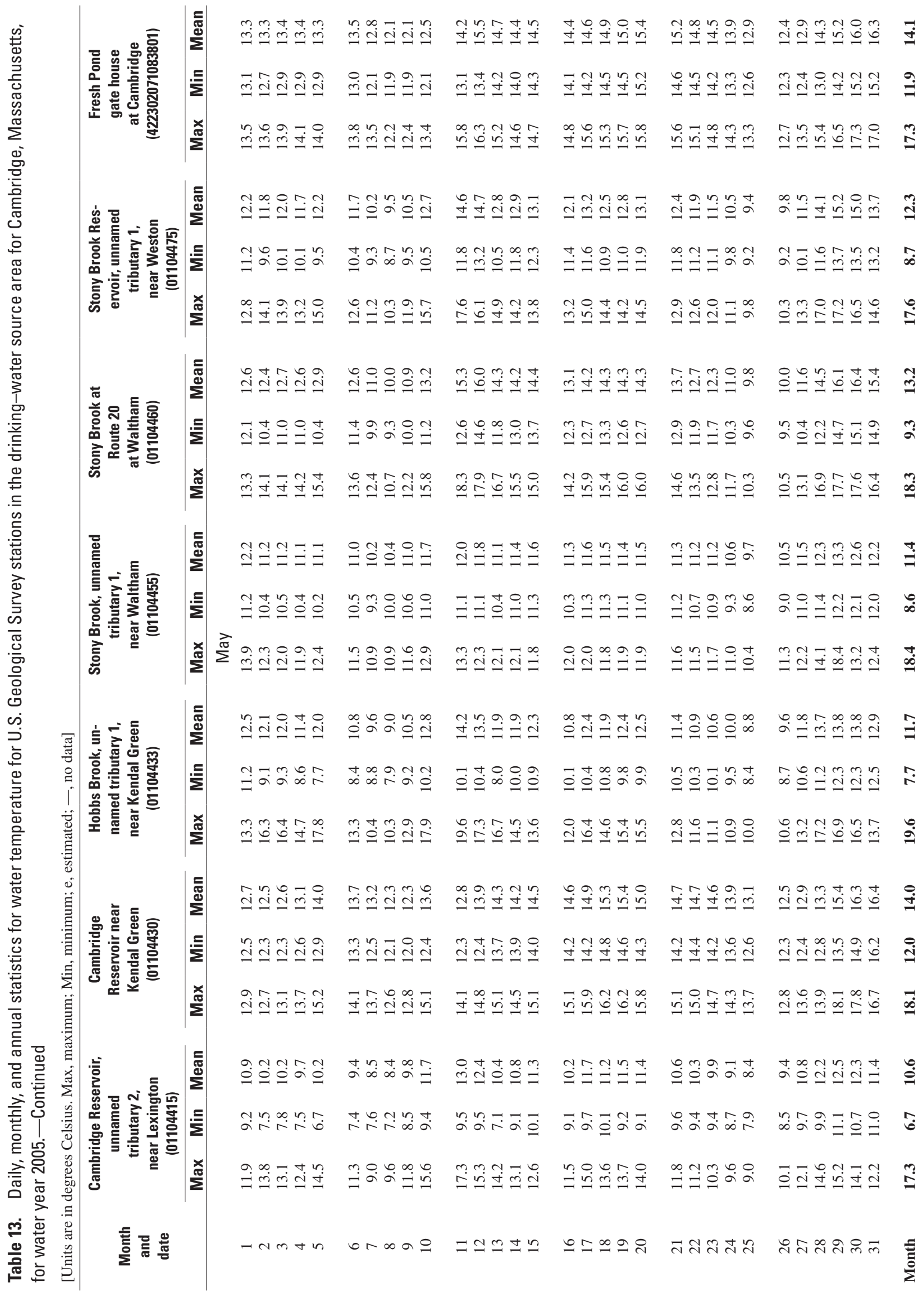




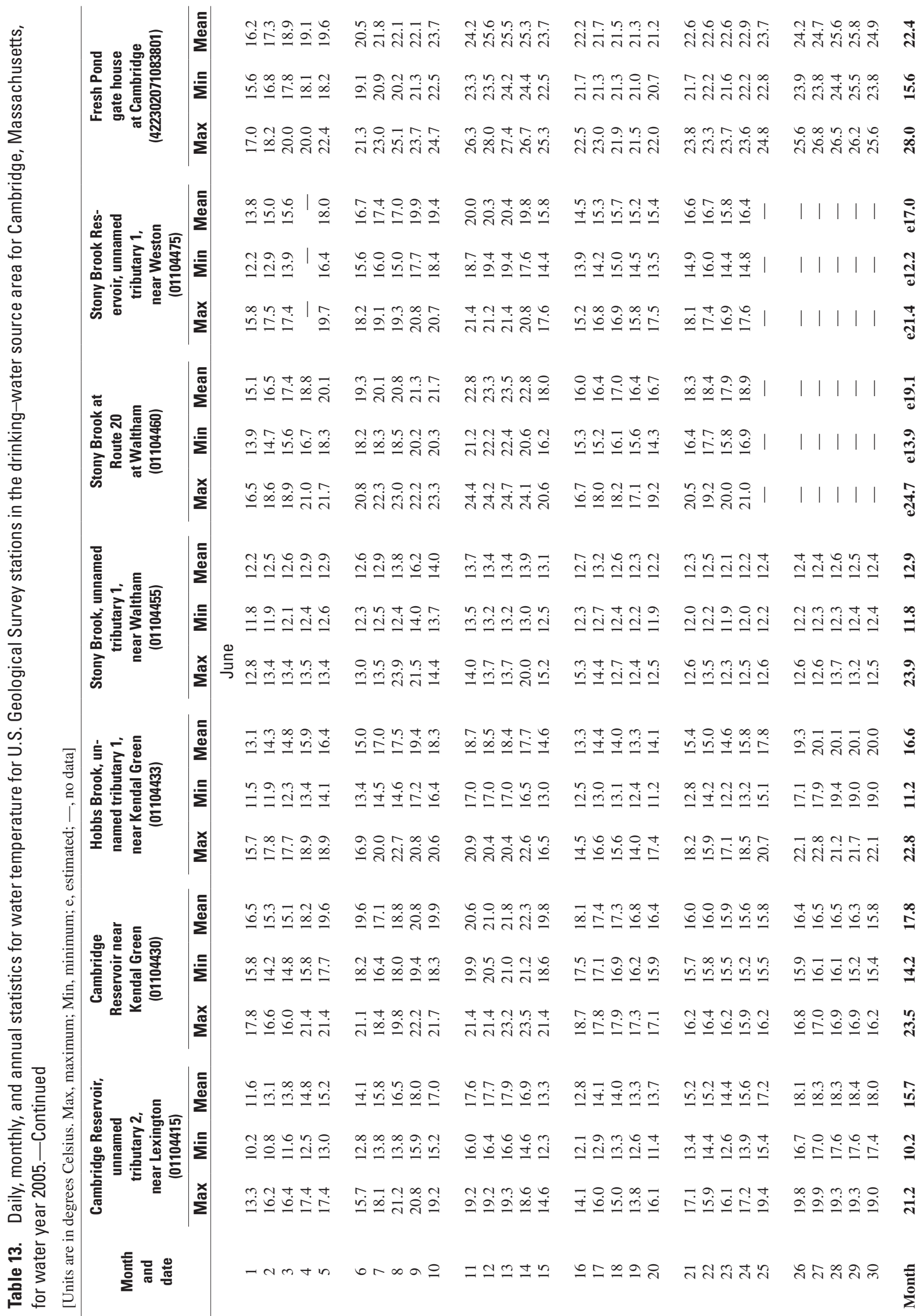




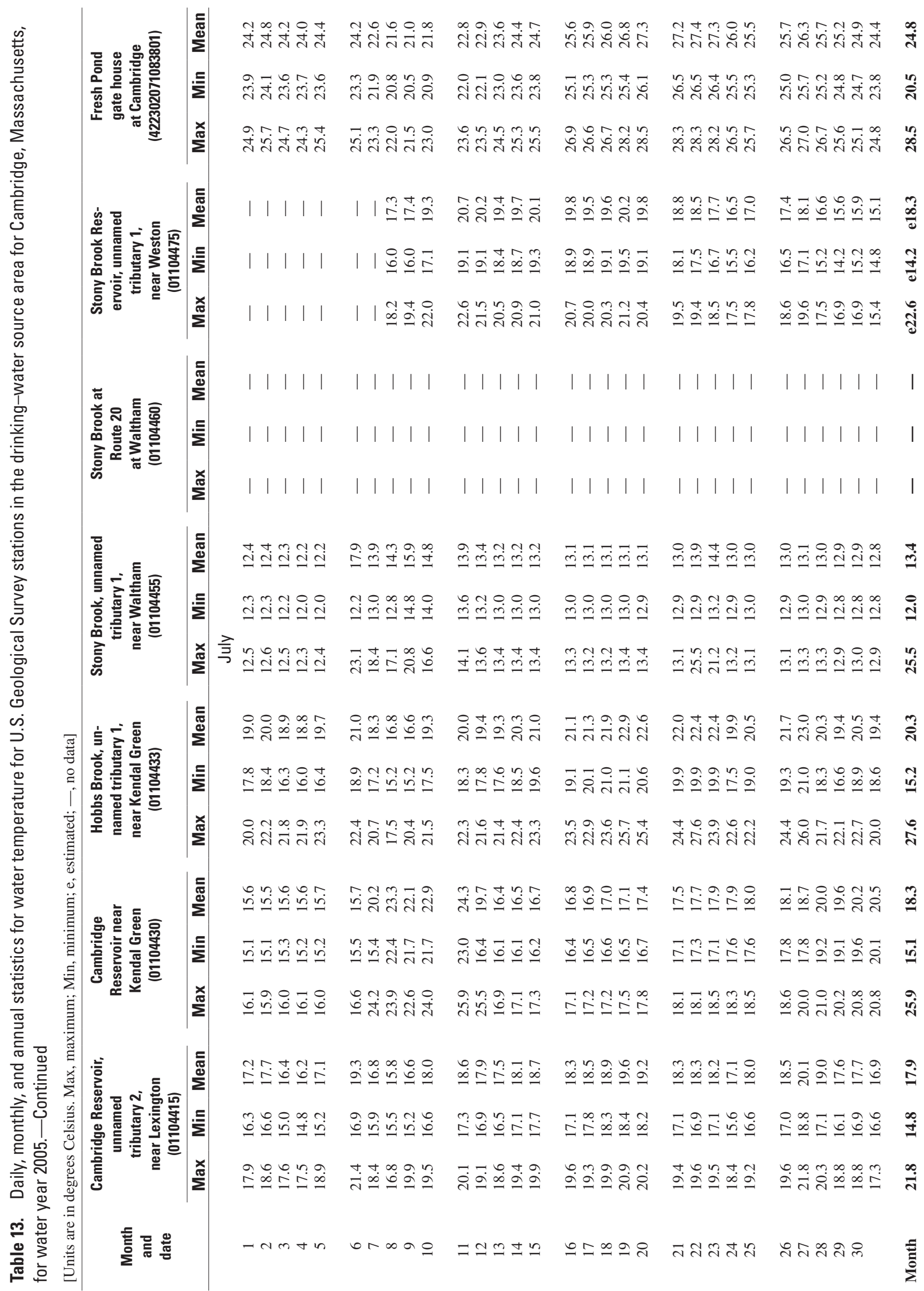




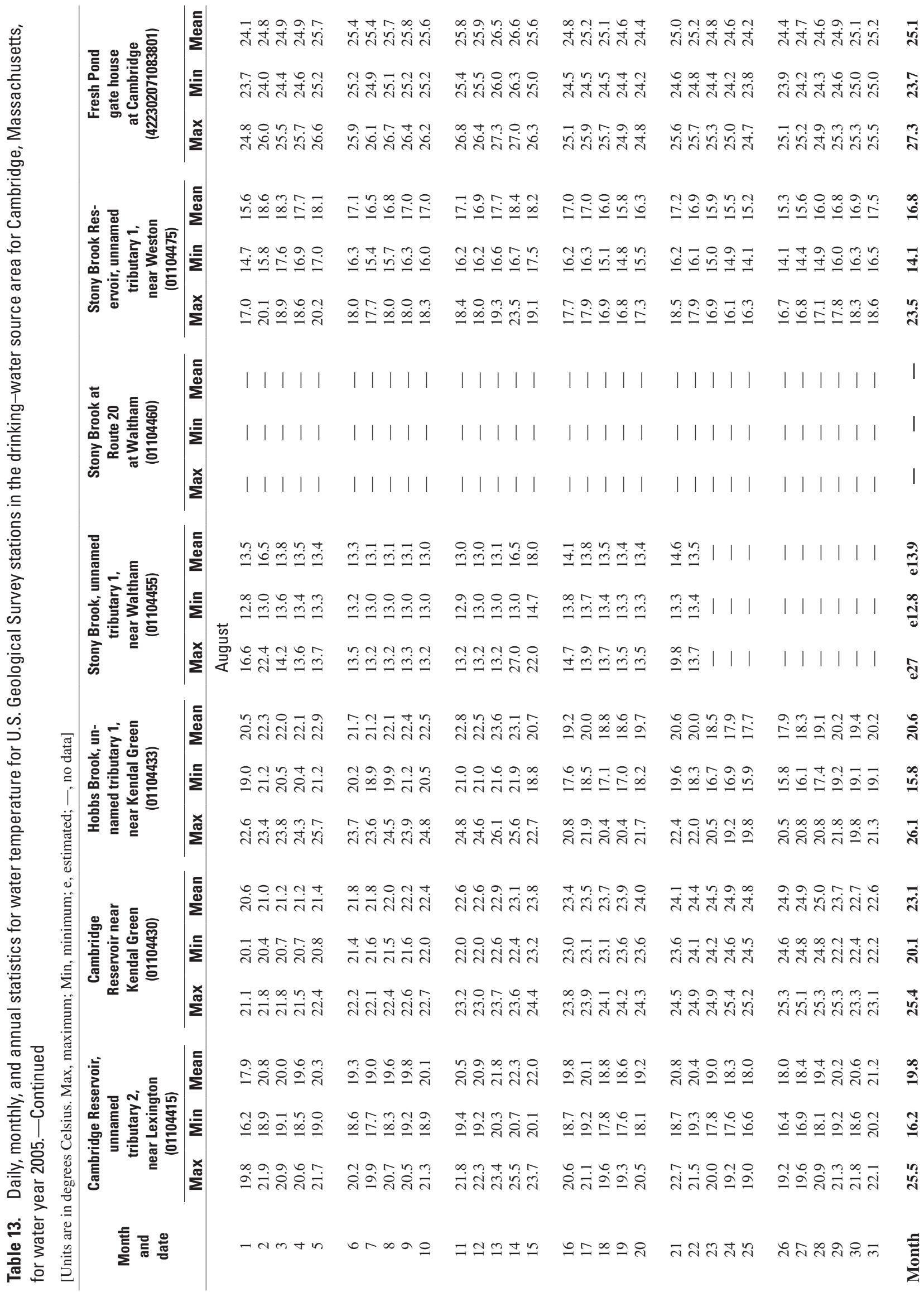




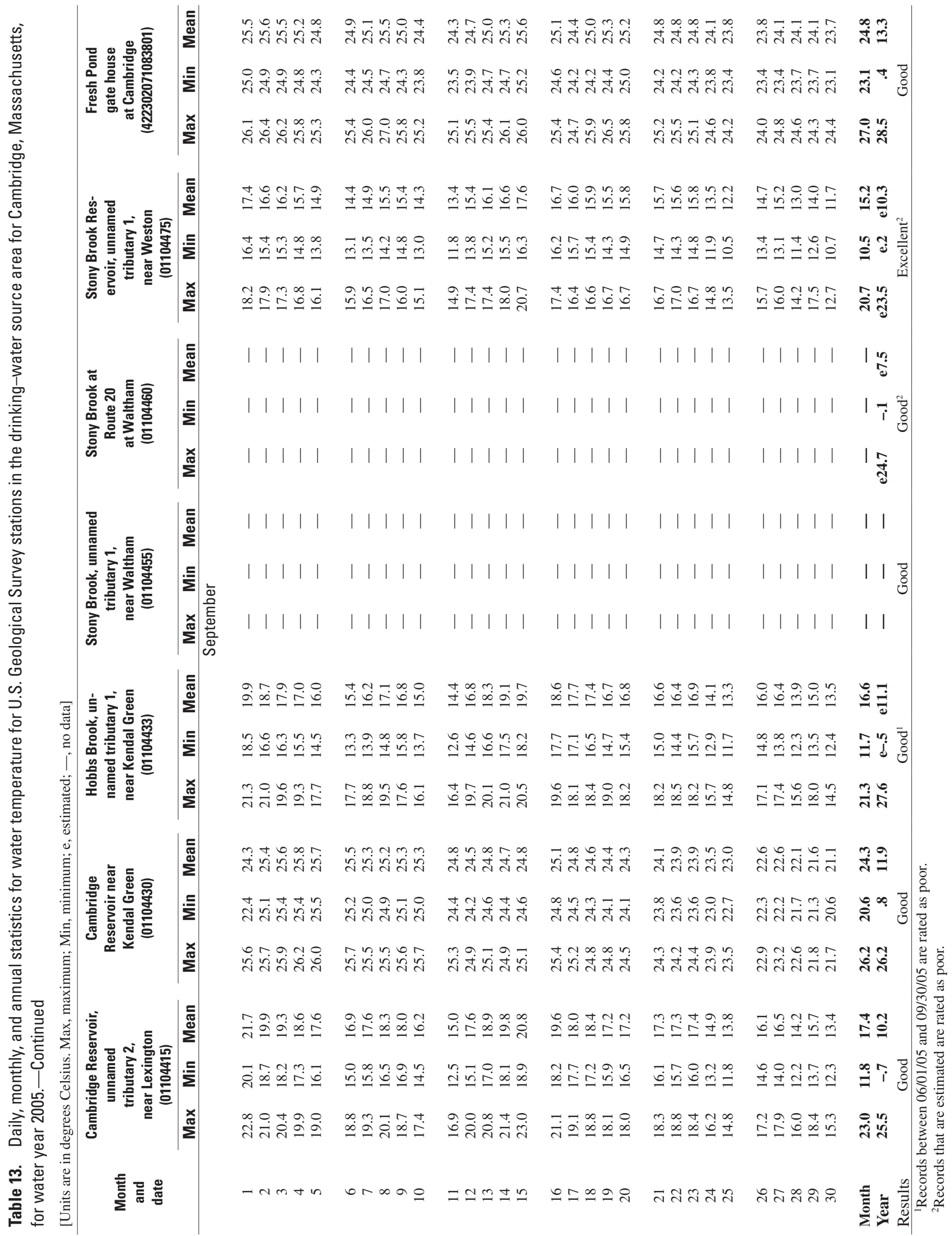




\begin{tabular}{|c|c|c|c|c|c|c|c|c|}
\hline${ }^{2}$ & 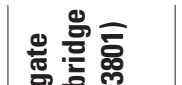 & $\sum_{\Sigma}^{\bar{\varpi}}$ & 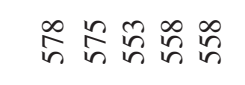 & 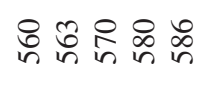 & 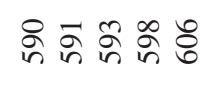 & 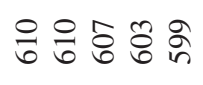 & 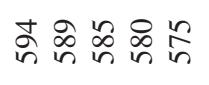 & 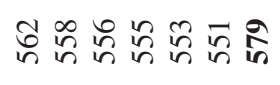 \\
\hline & 㤩 & $\underline{\underline{\Sigma}}$ & 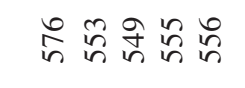 & 谣总总点 & 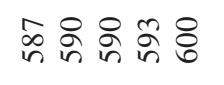 & 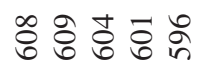 & 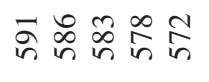 & 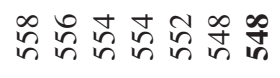 \\
\hline & 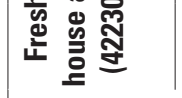 & 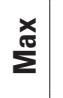 & 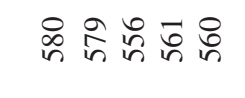 & 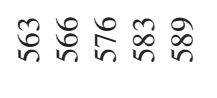 & 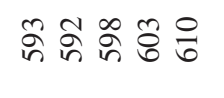 & تี تี & 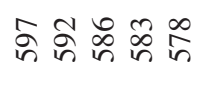 & 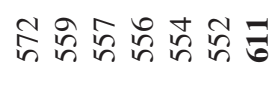 \\
\hline & 的昰离岕 & $\sum_{\Sigma}^{\overline{\mathbb{E}}}$ & 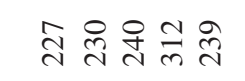 & 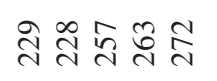 & 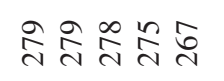 & สิ & 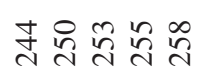 & 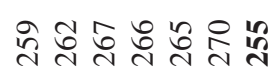 \\
\hline & 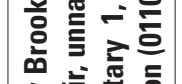 & $\underline{\Sigma}$ & 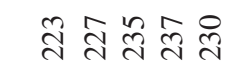 & 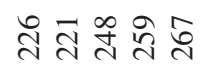 & 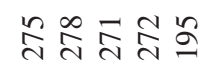 & छ & 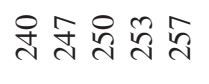 & 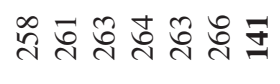 \\
\hline & 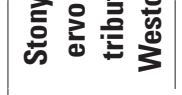 & $\begin{array}{l}\underset{x}{\mathbb{m}} \\
\sum^{\pi}\end{array}$ & సెત & 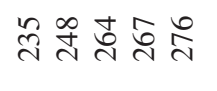 & ô & 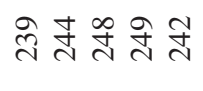 & 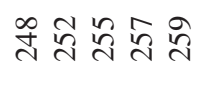 & ర్సं ठ্ণী : \\
\hline & 壳壱 & $\sum_{\Sigma}^{\bar{E}}$ & 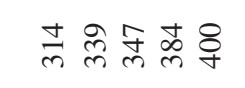 & 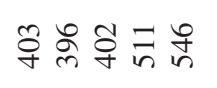 & 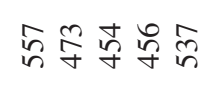 & 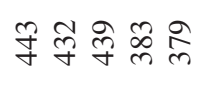 & 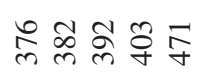 & 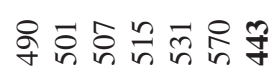 \\
\hline & & $\underline{\mathbf{E}}$ & 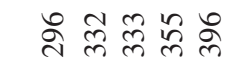 & 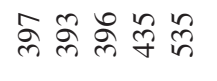 & 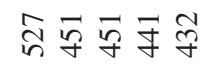 & હેને ન્ન & 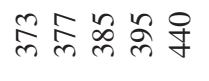 & \& \\
\hline & के & $\stackrel{\substack{x \\
\Sigma}}{m}$ & ભొ & 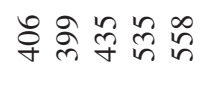 & 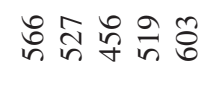 & 品品品字常 & 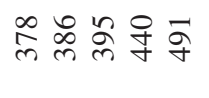 & 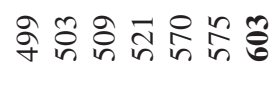 \\
\hline & 主总 & 离 & क & 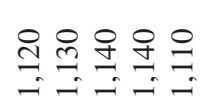 & 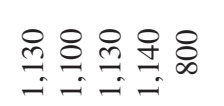 & 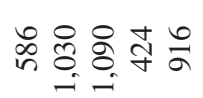 & 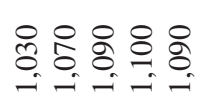 & 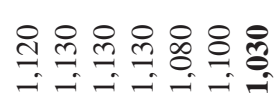 \\
\hline & 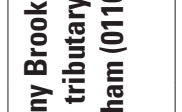 & $\underline{\Sigma}$ & œ & 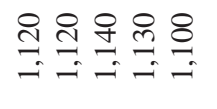 & 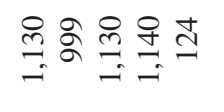 & 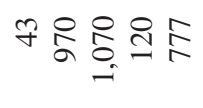 & 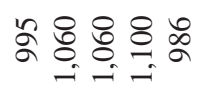 & 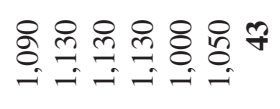 \\
\hline & 总 & 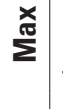 & 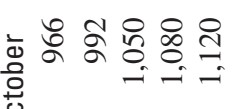 & 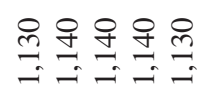 & 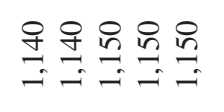 & 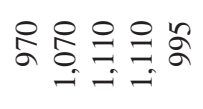 & $\begin{array}{l}8: 8 \% \\
8: 0\end{array}$ & 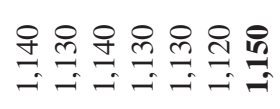 \\
\hline 䇂 & 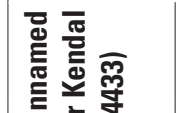 & 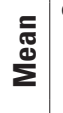 & 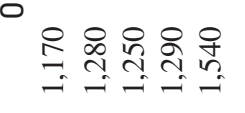 & 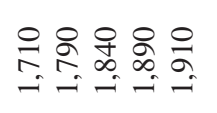 & 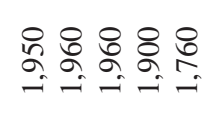 & 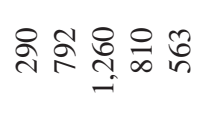 & 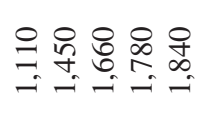 & 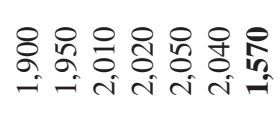 \\
\hline 音 & 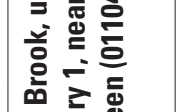 & E & 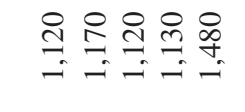 & 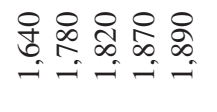 & 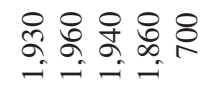 & 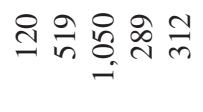 & 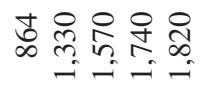 & 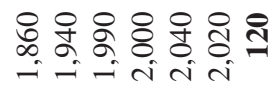 \\
\hline 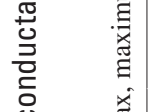 & 을 & $\stackrel{\substack{x \\
\Sigma}}{x}$ & 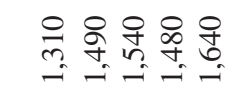 & 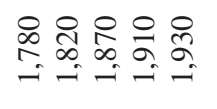 & 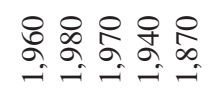 & 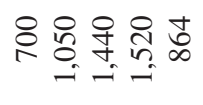 & 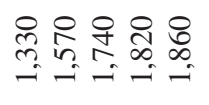 & 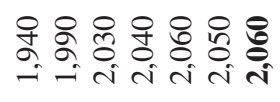 \\
\hline 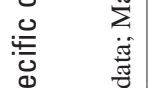 & 迹 즐 君 & $\sum_{\Sigma}^{\bar{\varpi}}$ & 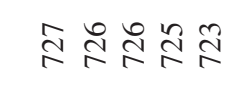 & 초ㅅㅠㅛ & 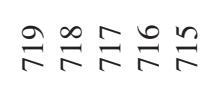 & ミㅠミミ & 융영 & 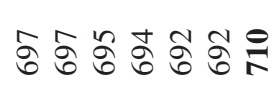 \\
\hline $\begin{array}{l}\stackrel{2}{\rightleftarrows} \\
i\end{array}$ & & $\underline{\Sigma}$ & ন্ন & 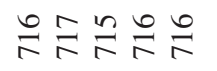 & 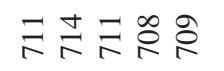 & 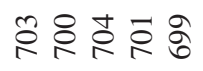 & 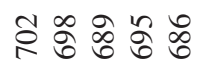 & $\hat{\sigma} \delta: \& \&$ \\
\hline 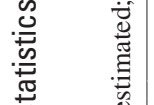 & & 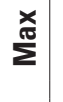 & 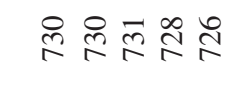 & 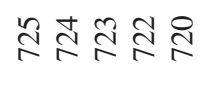 & 촗윳 $\stackrel{\infty}{*}$ & $\stackrel{2}{2} \stackrel{2}{2} \stackrel{2}{2}$ & 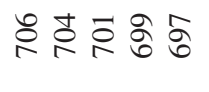 & 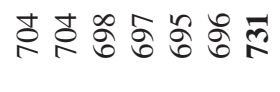 \\
\hline $\begin{array}{l}0 \\
\dot{0} \\
\ddot{\vec{\Delta}} \\
\dot{\Xi}\end{array}$ & 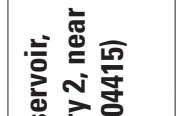 & $\stackrel{\bar{\varpi}}{\Sigma}$ & 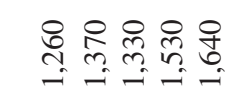 & 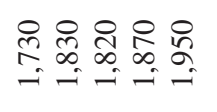 & 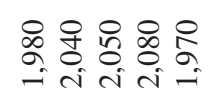 & 등요용요 & 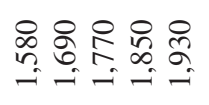 & 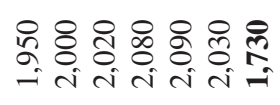 \\
\hline 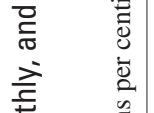 & 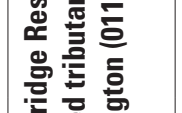 & $\bar{\Sigma}$ & 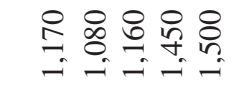 & 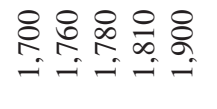 & 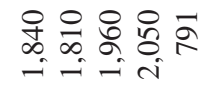 & 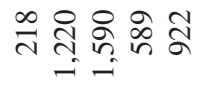 & 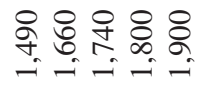 & 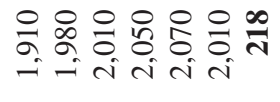 \\
\hline 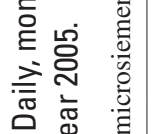 & 言密 & 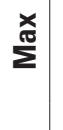 & 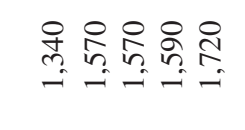 & 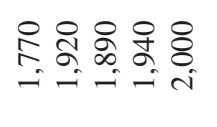 & 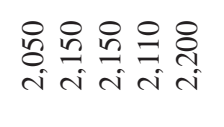 & 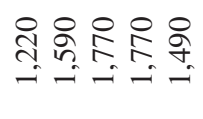 & $\begin{array}{l}0 \\
0 \\
0\end{array}$ & 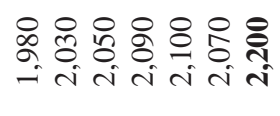 \\
\hline 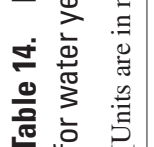 & 部营 & & & & $=s$ & & +2 & i \\
\hline
\end{tabular}




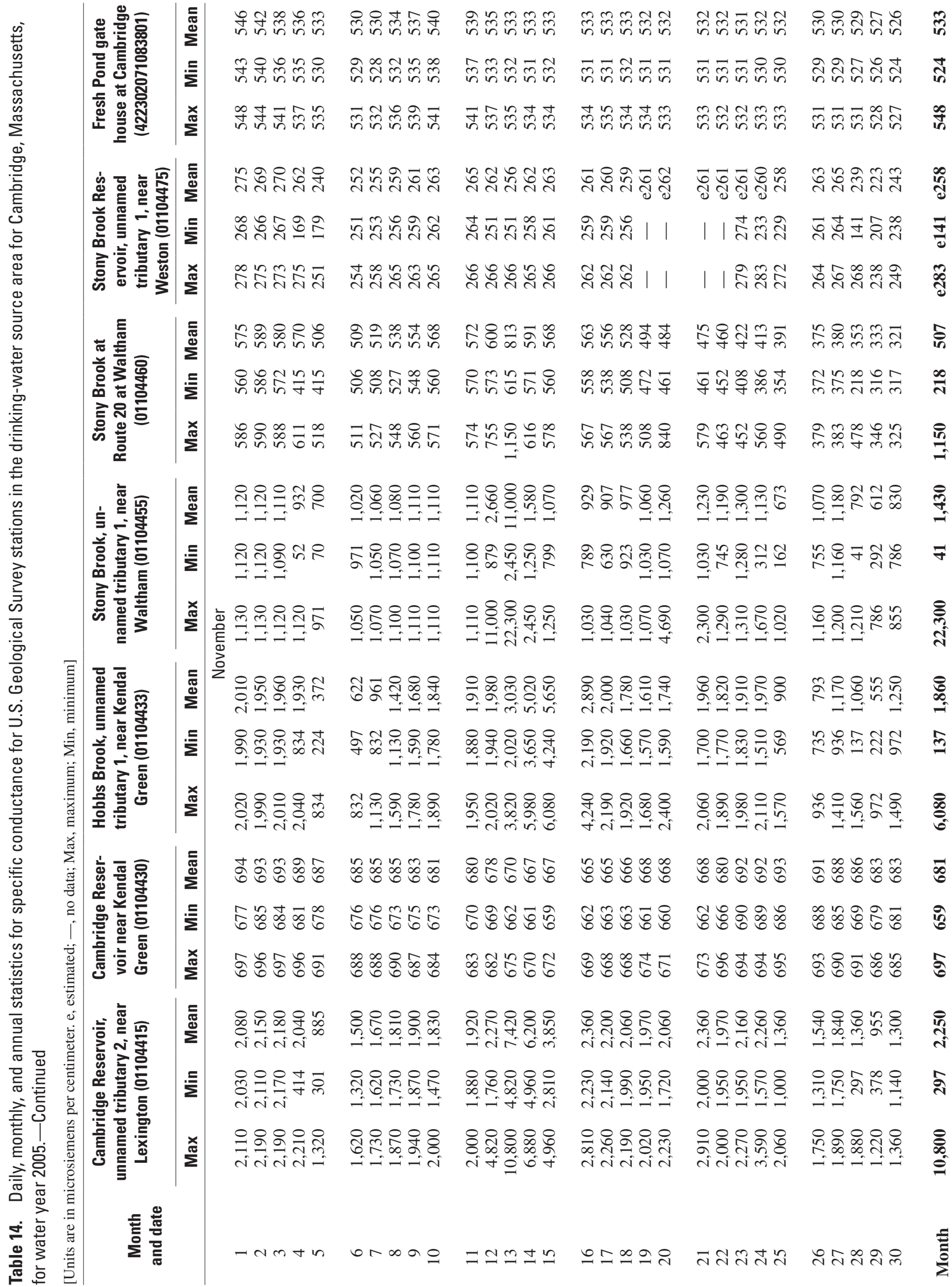




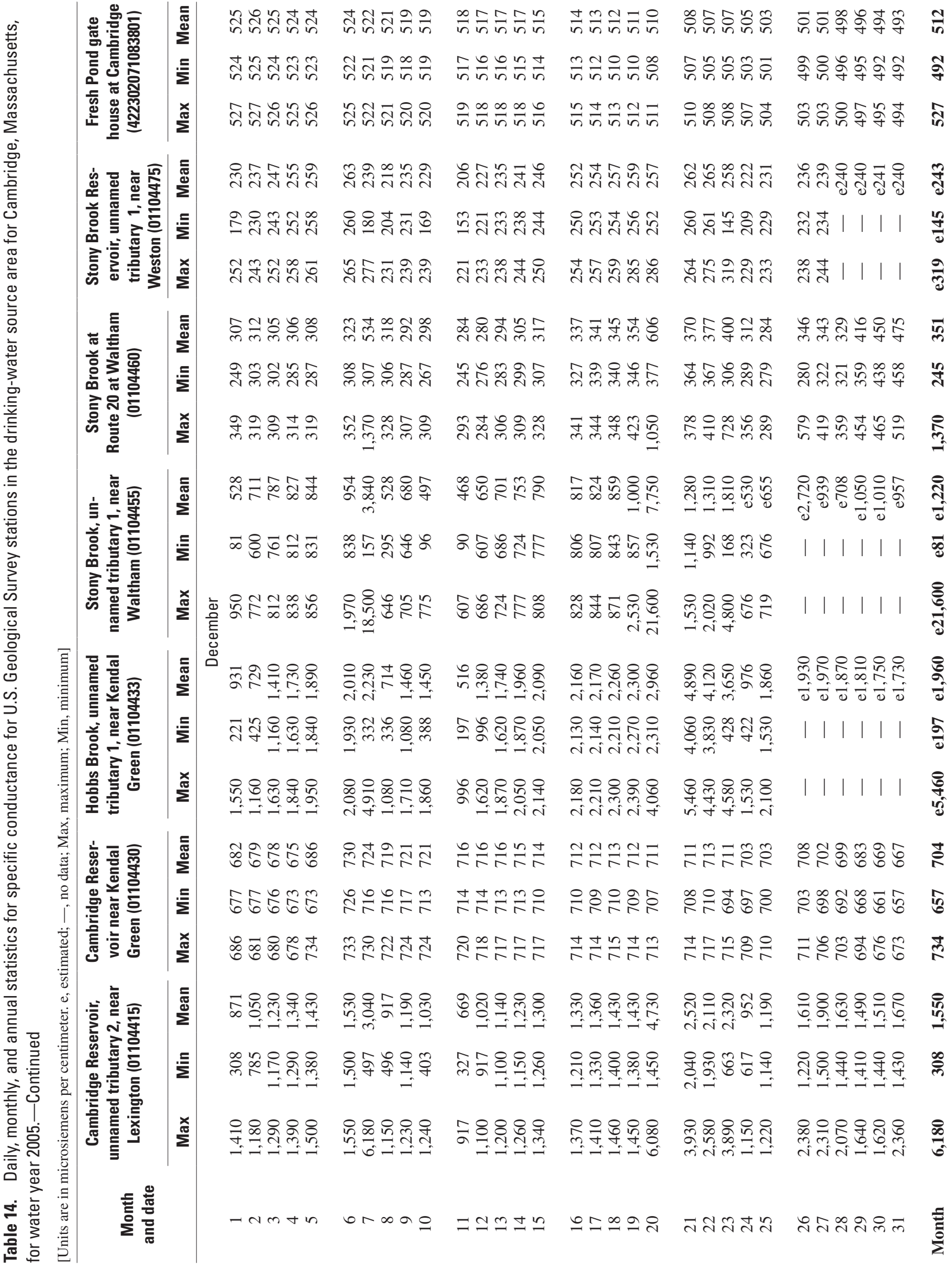




\begin{tabular}{|c|c|c|c|c|c|c|c|c|}
\hline 5 & 莺 淧 & 恶 & 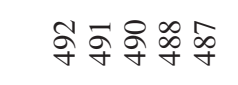 & 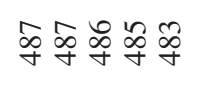 & ஜ̂ & 各宇宇守寺 & 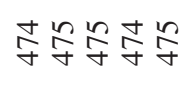 & 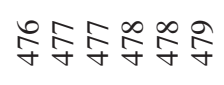 \\
\hline & 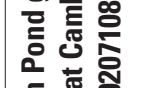 & E⿳亠二口犬 & 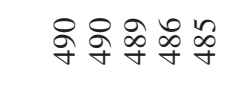 & \& & 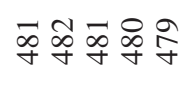 & 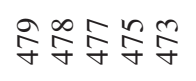 & 孚孛孛夺孚 & 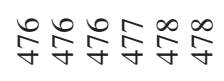 \\
\hline & 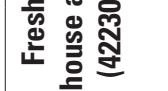 & $\stackrel{x}{\stackrel{x}{\Sigma}}$ & 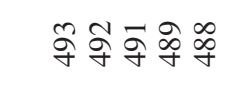 & 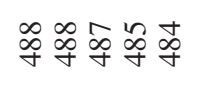 & 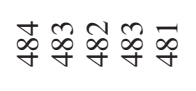 & 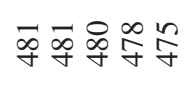 & 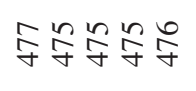 & 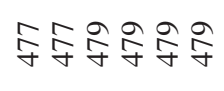 \\
\hline & 的㤩点 & 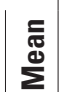 & 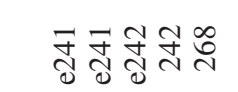 & 오윯초솣 & 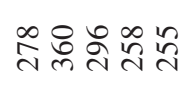 & 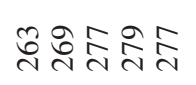 & 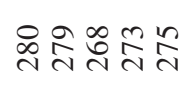 & 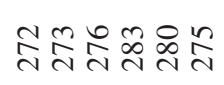 \\
\hline & 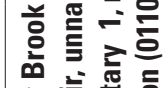 & 产 & 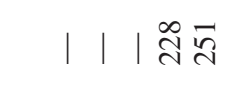 & 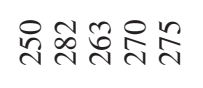 & 유용 & 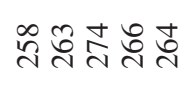 & 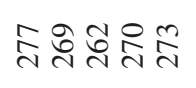 & 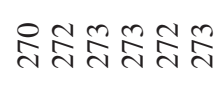 \\
\hline & 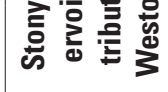 & $\begin{array}{l}x \\
\prod^{x} \\
\Sigma\end{array}$ & | | | 弅 & mi & 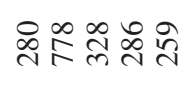 & 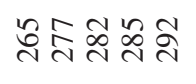 & $\underset{\infty}{\mathbb{N}} \stackrel{\infty}{\sim} \vec{\sim} \underset{\sim}{\infty} \underset{\sim}{\infty}$ & 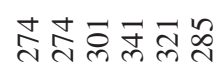 \\
\hline & ๘ & 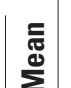 & 号守寻专等 & 守尽乩方京 & 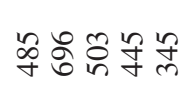 & 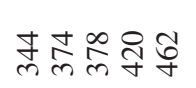 & 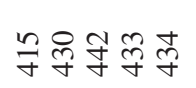 & 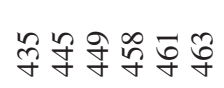 \\
\hline & 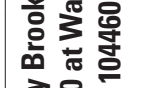 & $\bar{\Sigma}$ & 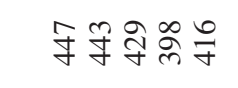 & 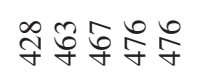 & 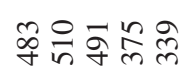 & 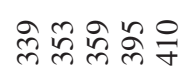 & 악 & 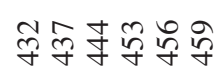 \\
\hline & 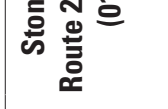 & $\stackrel{x}{\underset{m}{\Sigma}}$ & 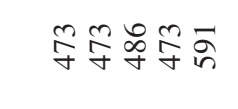 & 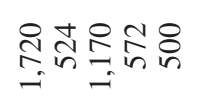 & 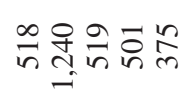 & 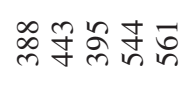 & 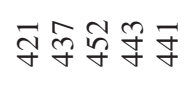 & 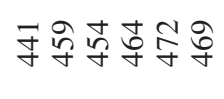 \\
\hline & 息总疍 & 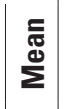 & 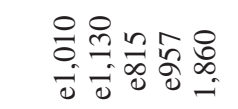 & 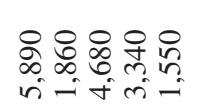 & 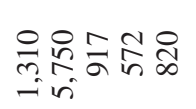 & 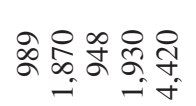 & 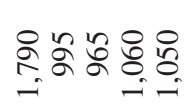 & 스용의 \\
\hline & & $\underline{\Sigma}$ & || $\mid \stackrel{\infty}{\stackrel{\infty}{\infty}}$ & 웅응ㅇㅇㅇ영 & 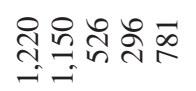 & 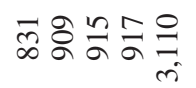 & 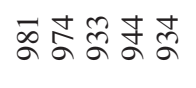 & 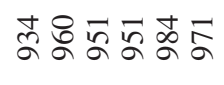 \\
\hline & 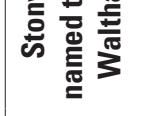 & $\stackrel{x}{\underset{m}{\Sigma}}$ & 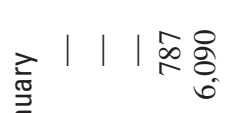 & 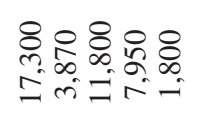 & 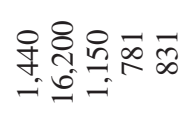 & 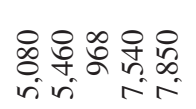 & 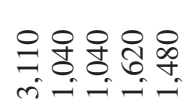 & 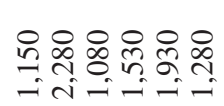 \\
\hline 㹂 & 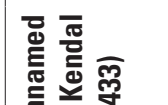 & $\underset{\bar{\varpi}}{\stackrel{\Xi}{\Sigma}}$ & 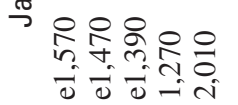 & 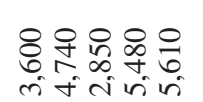 & 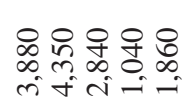 & 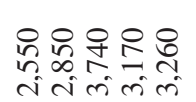 & 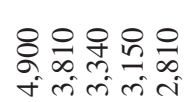 & 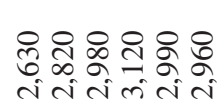 \\
\hline$\sum_{\dot{E}}$ & 尊 & $\underline{\mathbf{E}}$ & | 1 | & 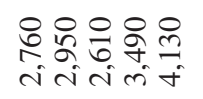 & 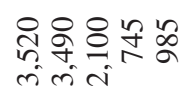 & 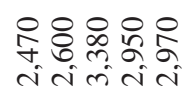 & 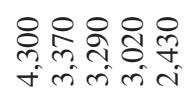 & 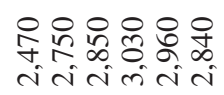 \\
\hline 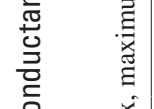 & 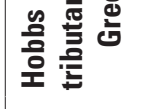 & $\stackrel{x}{\coprod^{m}}$ & 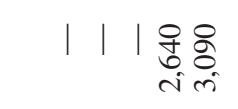 & 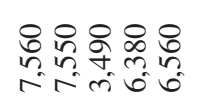 & 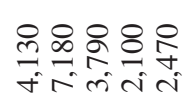 & 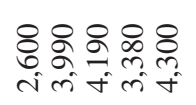 & 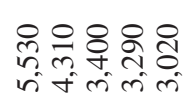 & 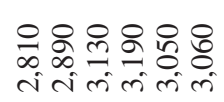 \\
\hline 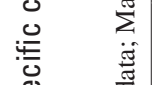 & 迹 즐 웜 & 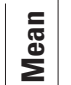 & 창융융융 & : & : & 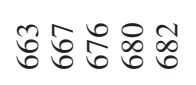 & 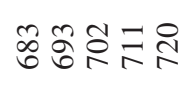 & 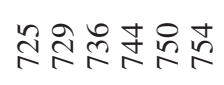 \\
\hline & 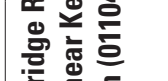 & $\underline{\Sigma}$ & 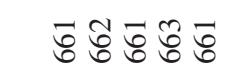 & 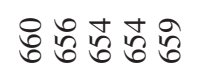 & 8 & 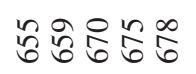 & 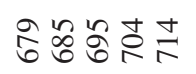 & 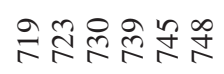 \\
\hline 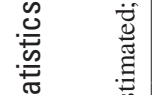 & 言 & $\stackrel{x}{\substack{m \\
\Sigma}}$ & โิธิ์ธำ & 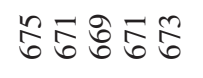 & 苂递茴志芯 & 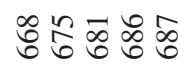 & 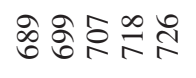 & 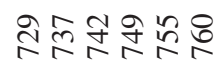 \\
\hline $\begin{array}{l}0 \\
\dot{0} \\
\ddot{\vec{u}} \\
\dot{0}\end{array}$ & 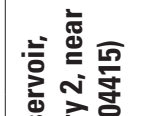 & 离 & 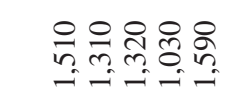 & 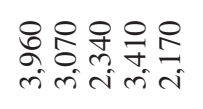 & 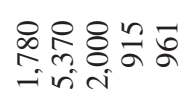 & 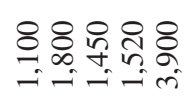 & 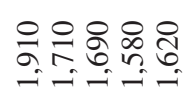 & 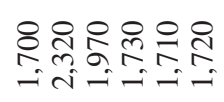 \\
\hline 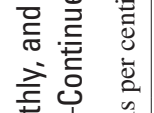 & 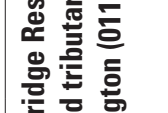 & 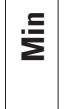 & శ్లిల్లిః方 రి & 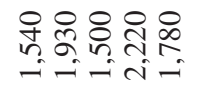 & 응요 & 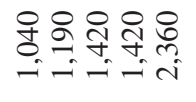 & & $\begin{array}{ll}8 & 0 \\
0 & 0\end{array}$ \\
\hline 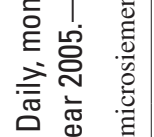 & 㤩高 & 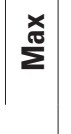 & 규ㅇㅛㅠ유유. & 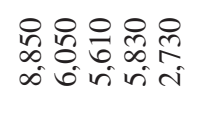 & 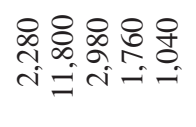 & 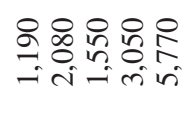 & 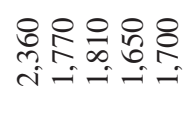 & 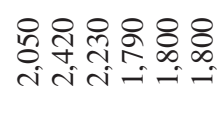 \\
\hline 总 & ¿̄ & & & & & & & \\
\hline
\end{tabular}




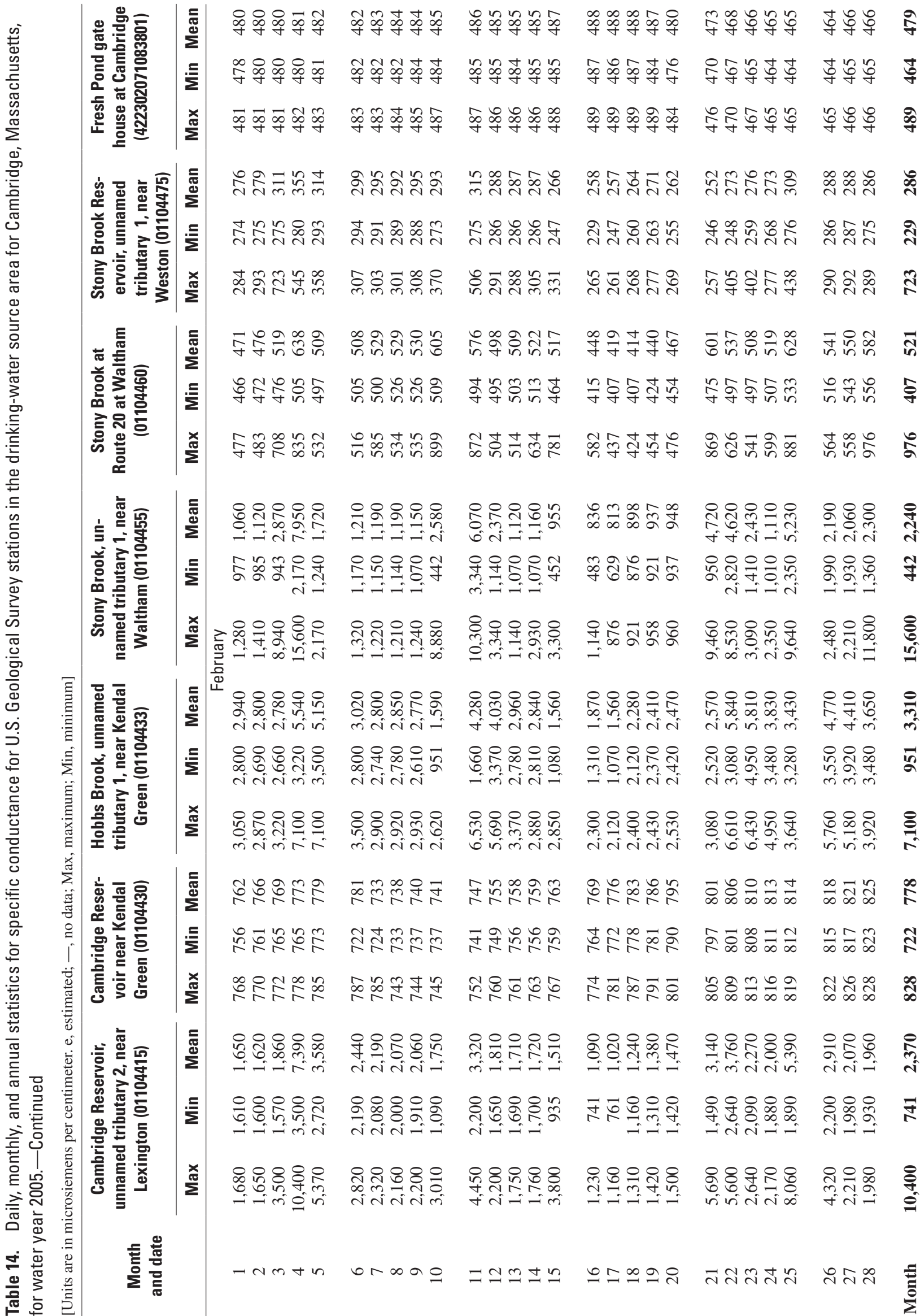




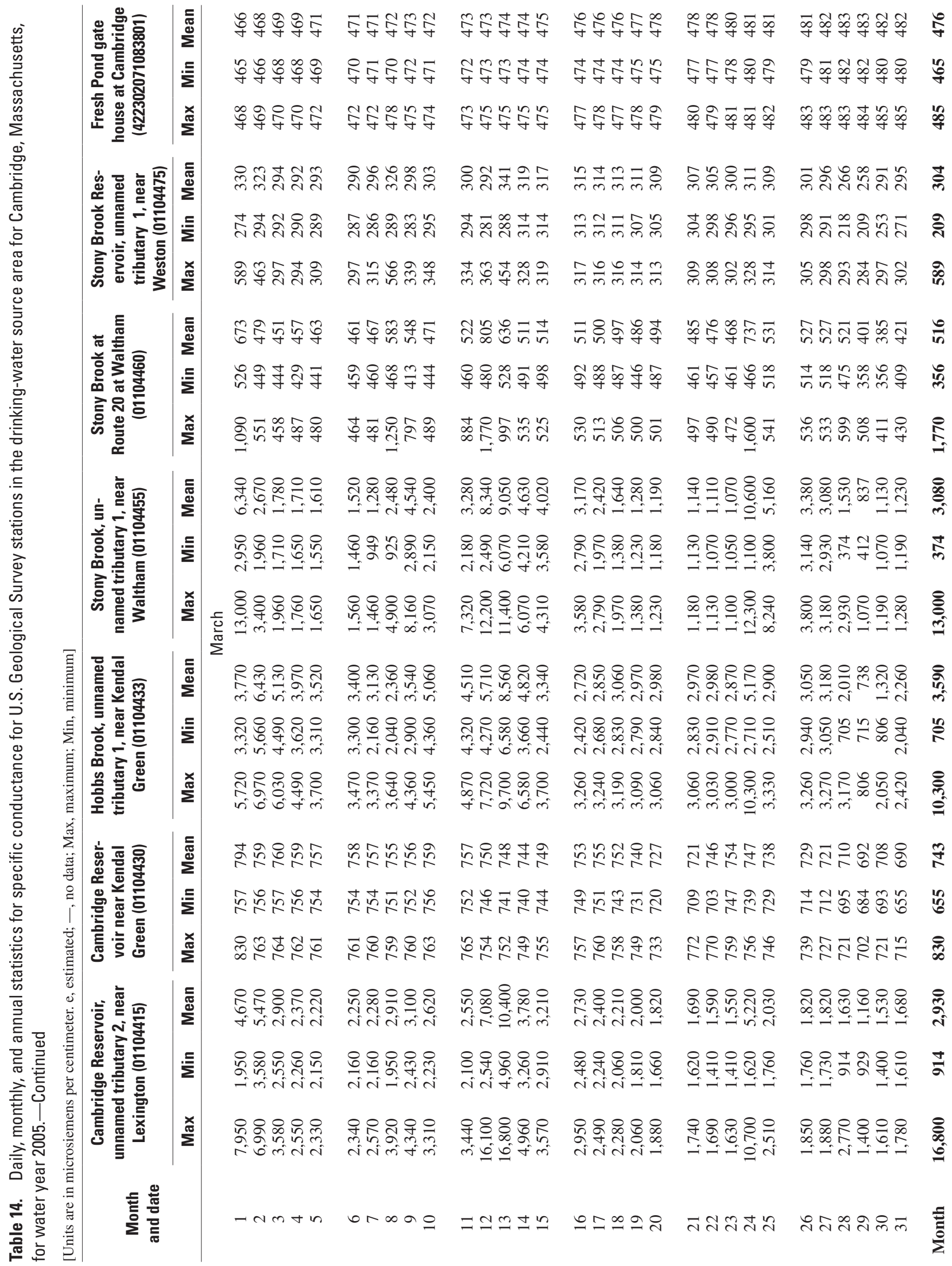




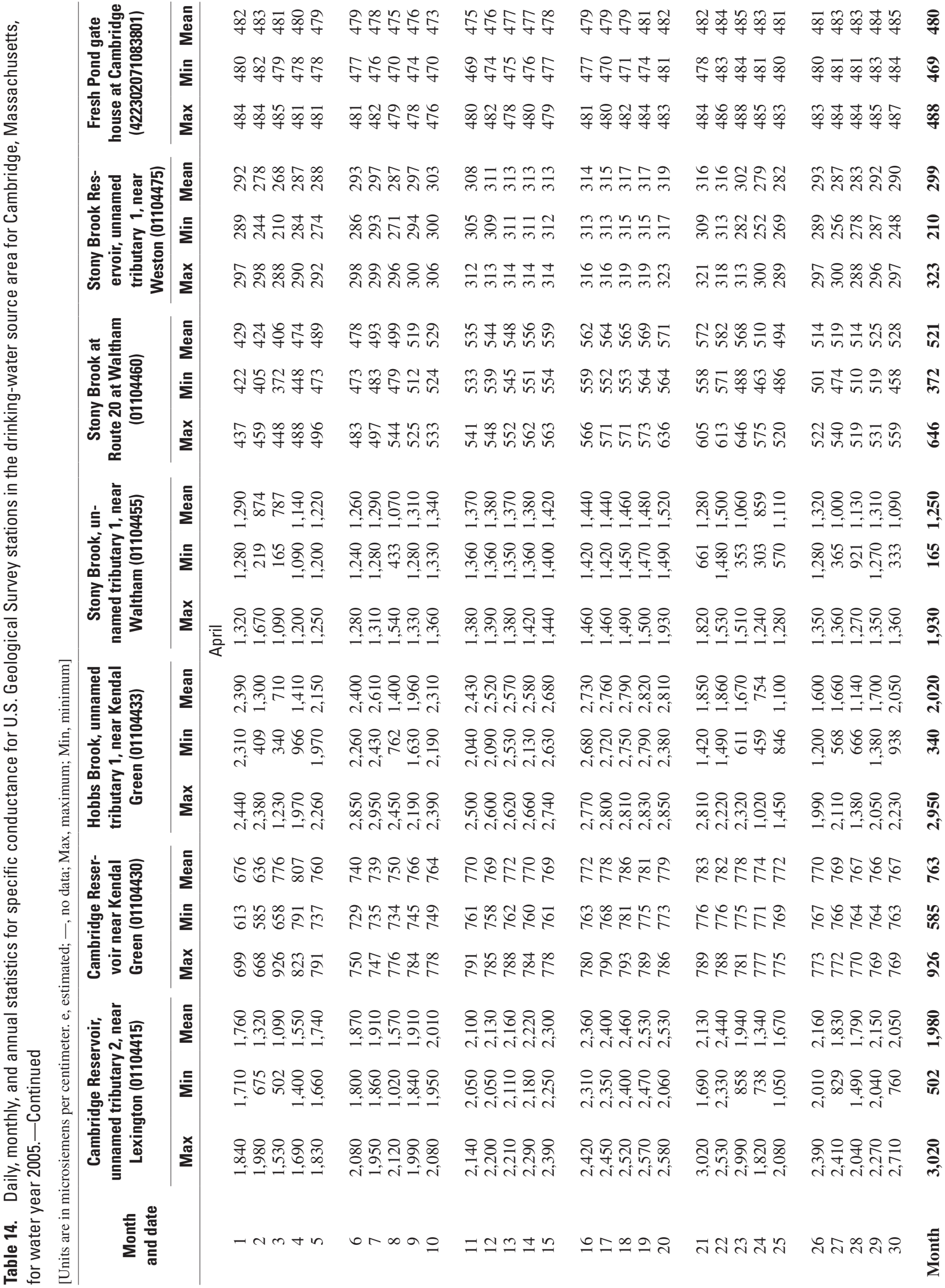




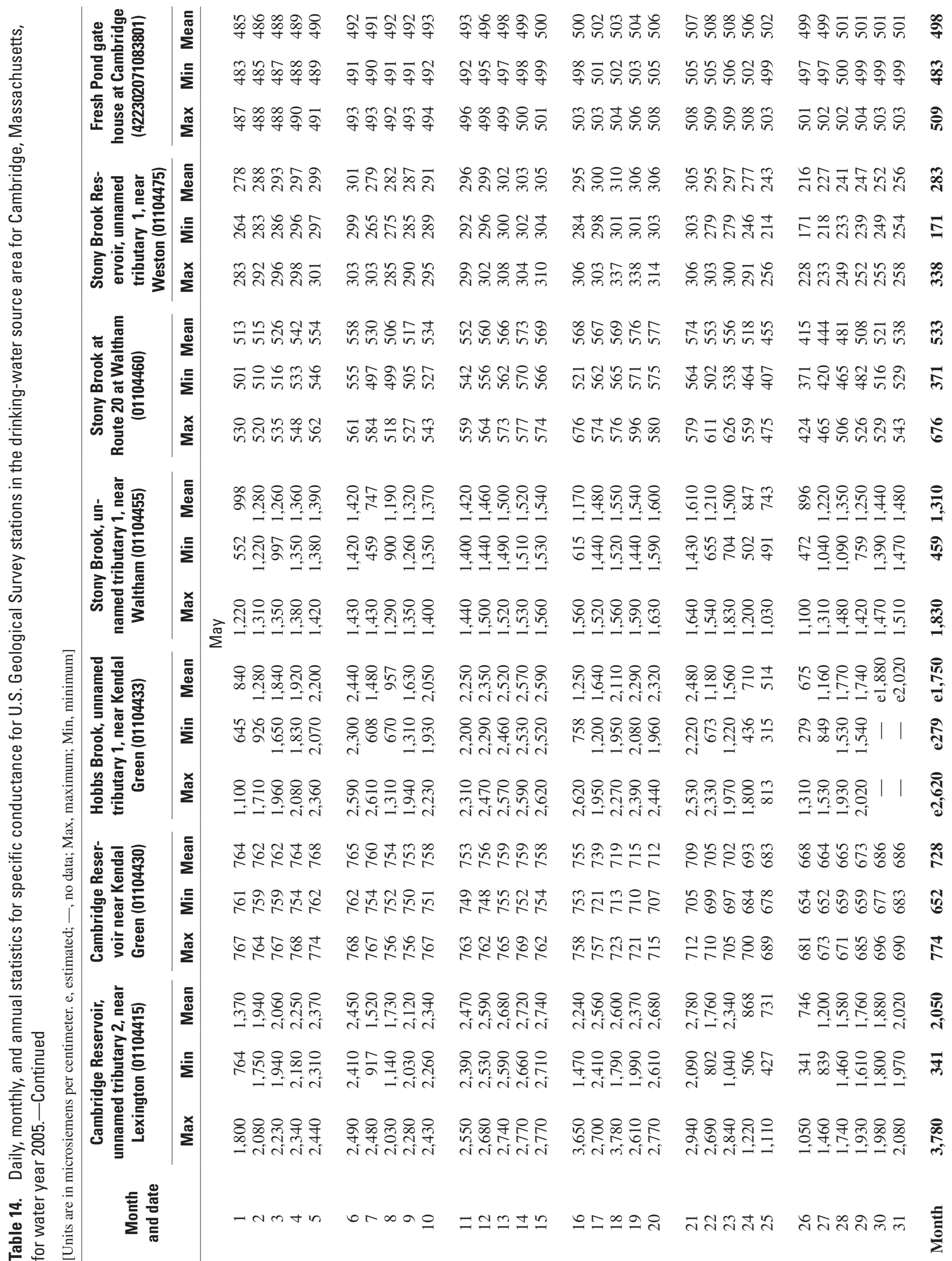




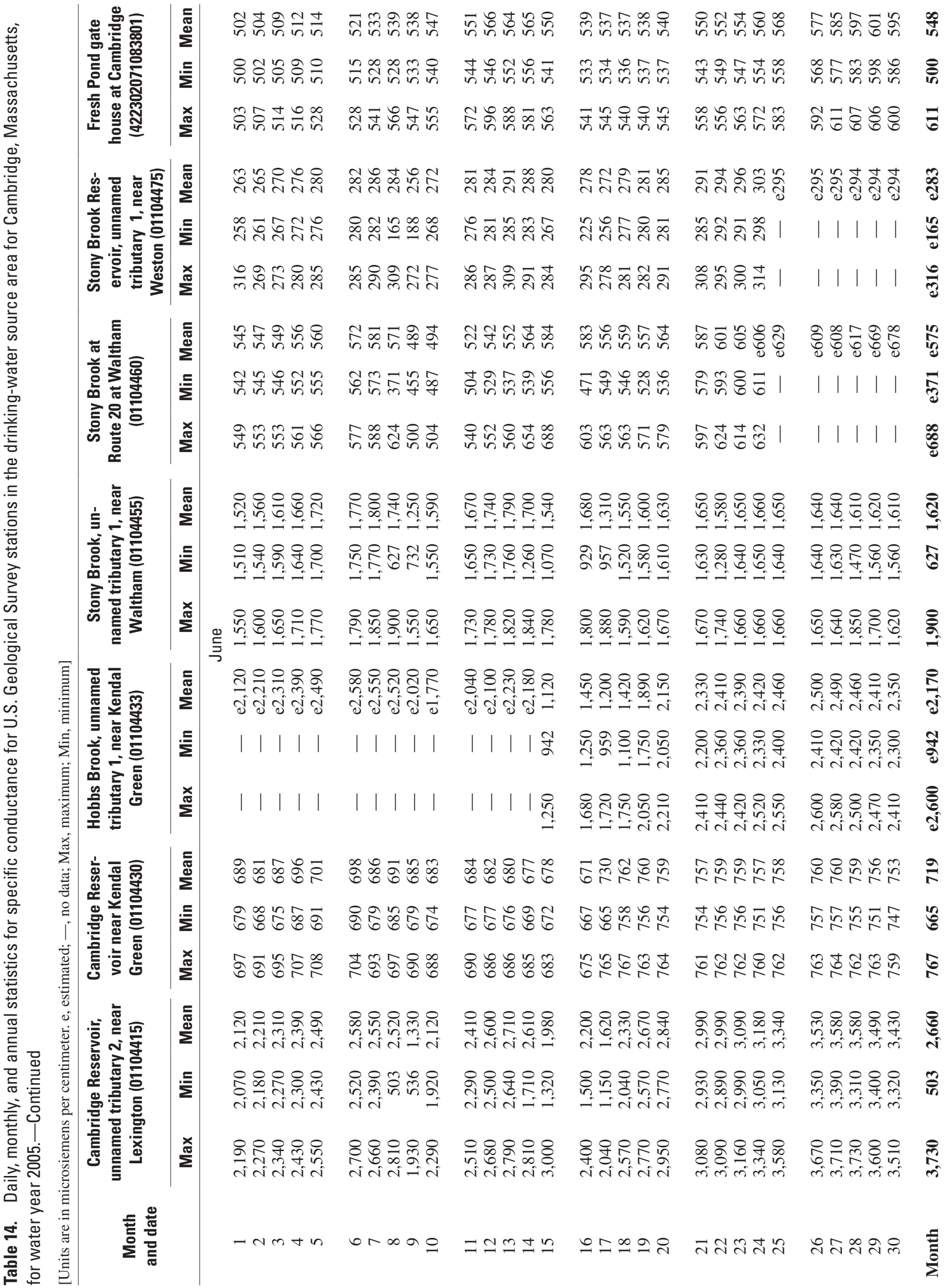




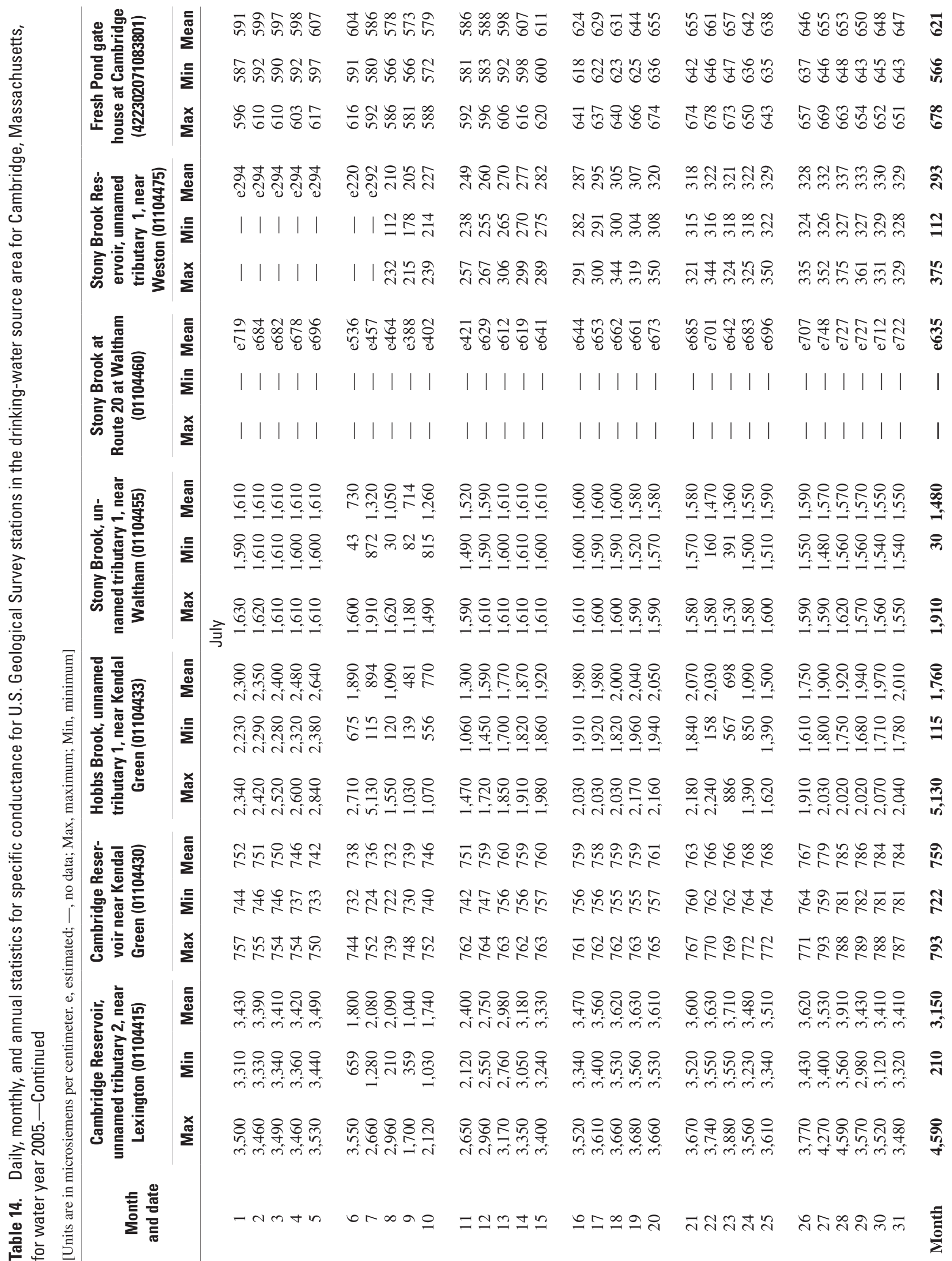




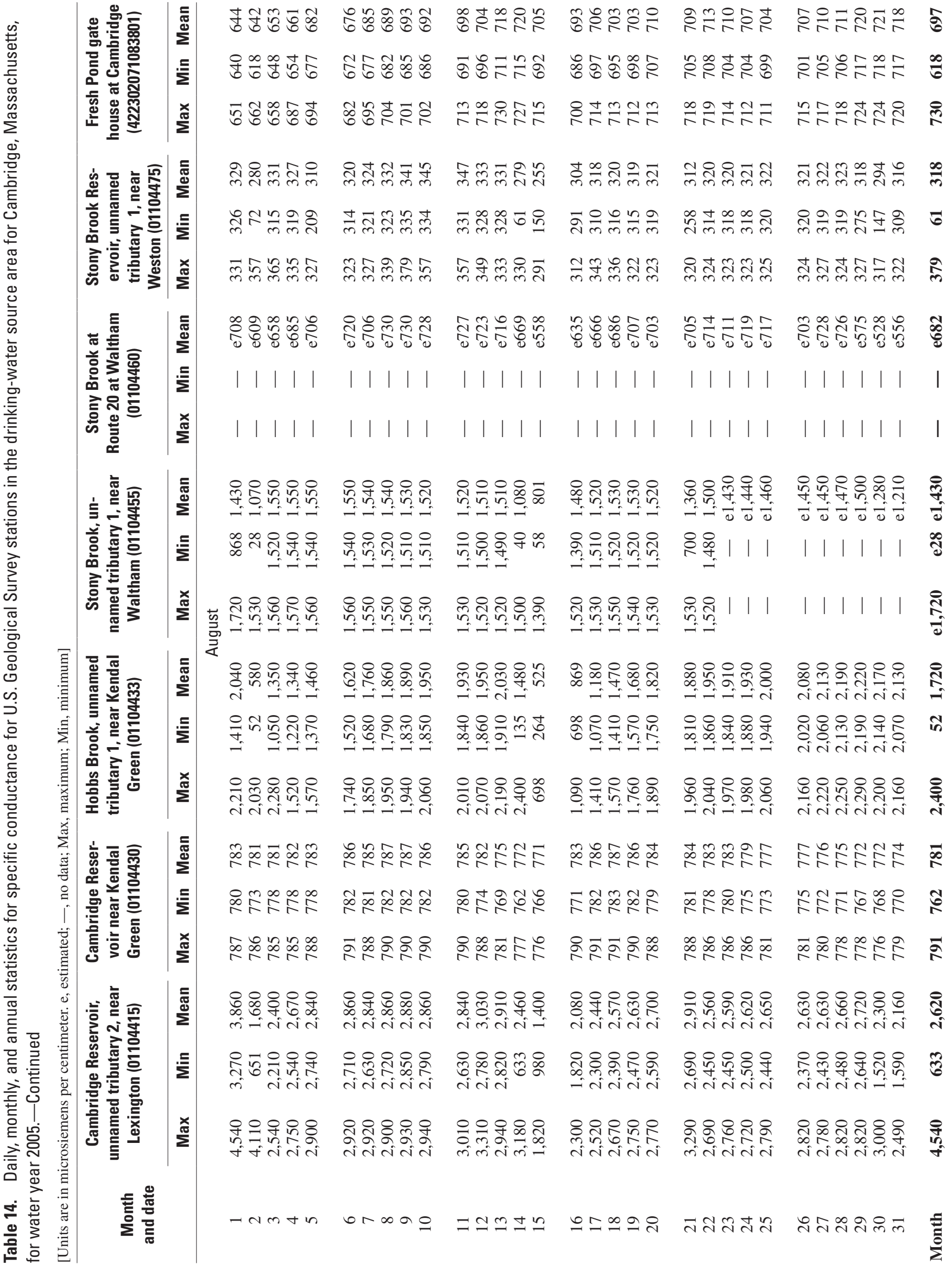




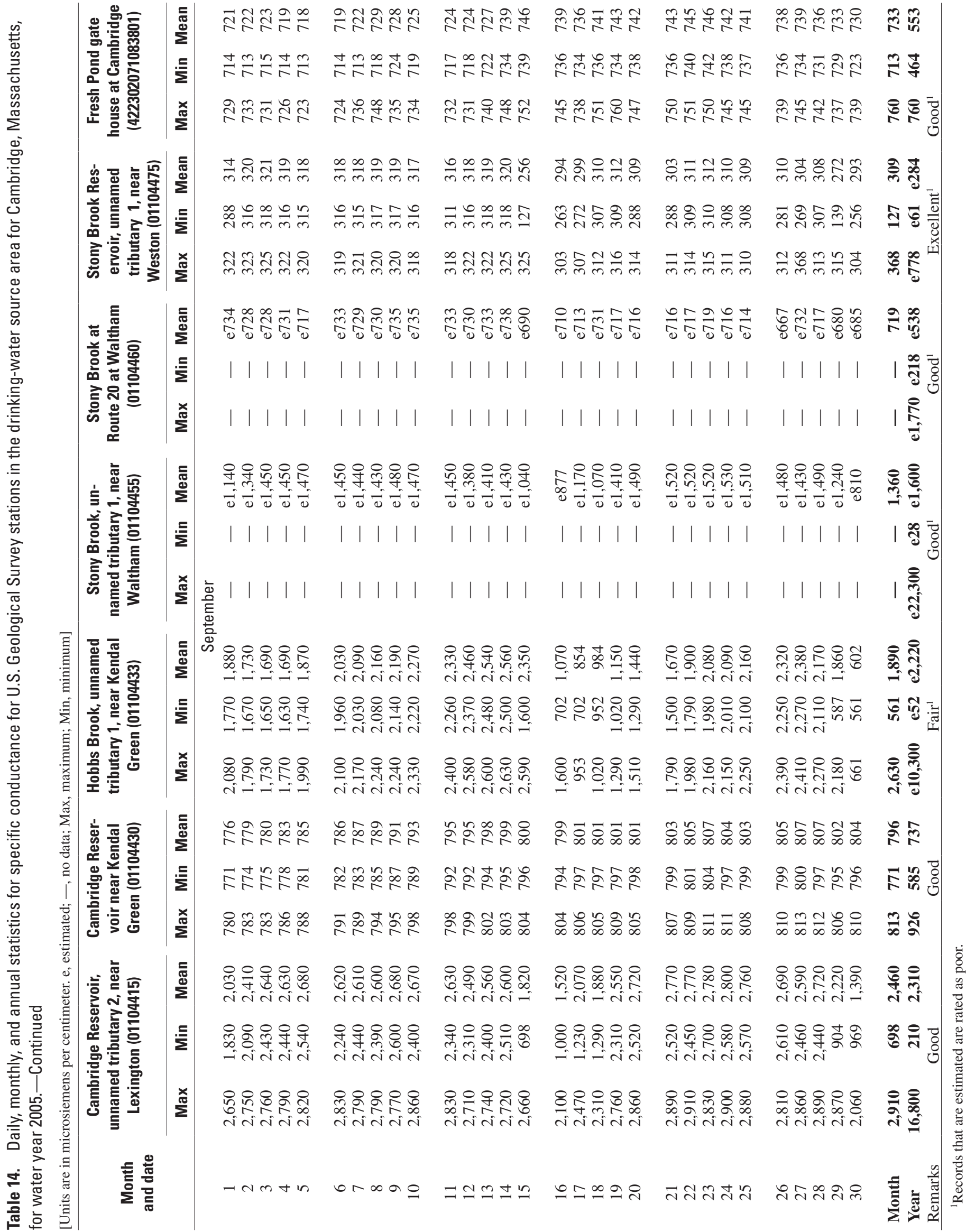


Table 15. Physical properties and concentrations of major inorganic constituents, nutrients, trace metals, suspended sediments, Escherichia coli bacteria, polyaromatic hydrocarbons, and polar pesticides and metabolites for base-flow and stormflow water samples collected in four subbasins and the Fresh Pond intake structure in the Cambridge, Massachusetts, drinking-water source area for water year 2005.

[Escherichia coli concentrations were mathematically estimated from subcomposites. PXXXXX, National Water Quality Laboratory parameter code; ${ }^{\circ} \mathrm{C}$, degree Celsius; e, estimated; NTRU, nephelometric turbidity ratio units; USGS-MA-LAB, U.S. Geological Survey-Massachusetts Water Science Center laboratory; <, concentration is less than value shown; id, insufficient data to estimate composite value; - , no data]

\begin{tabular}{|c|c|c|c|c|c|c|c|c|}
\hline $\begin{array}{l}\text { USGS station } \\
\text { number }\end{array}$ & $\begin{array}{l}\text { Sample } \\
\text { type }\end{array}$ & Begin date & Begin time & End date & End time & $\begin{array}{c}\text { Discharge, } \\
\text { instantaneous } \\
\text { (million gal- } \\
\text { lons per day) } \\
\text { P50051 }\end{array}$ & $\begin{array}{l}\text { Turbidity, } \\
\text { water, unfil- } \\
\text { tered (NTRU) } \\
\text { P63676 }\end{array}$ & $\begin{array}{c}\text { Dissolved } \\
\text { oxygen, wa- } \\
\text { ter, unfiltered } \\
\text { (milligrams } \\
\text { per liter) } \\
\text { P00300 }\end{array}$ \\
\hline 01104415 & Base flow & 20041215 & 1025 & - & - & 0.47 & 1.7 & 11.4 \\
\hline 01104415 & Stormflow & 20050708 & 1037 & 20050709 & 759 & - & 40 & - \\
\hline 01104415 & Base flow & 20050726 & 1210 & - & - & .01 & 1.1 & 5.3 \\
\hline 01104415 & Stormflow & 20050814 & 1751 & 20050815 & 1157 & - & 79 & - \\
\hline 01104433 & Base flow & 20041215 & 1205 & - & - & .13 & 1.8 & 8.9 \\
\hline 01104433 & Stormflow & 20050708 & 1126 & 20050709 & 900 & - & 20 & - \\
\hline 01104433 & Base flow & 20050726 & 1245 & - & - & & 3.2 & 4.2 \\
\hline 01104433 & Stormflow & 20050915 & 1008 & 20050916 & 215 & - & 45 & - \\
\hline 01104455 & Base flow & 20041215 & 1355 & - & - & .63 & .6 & 9.7 \\
\hline 01104455 & Stormflow & 20050708 & 1005 & 20050709 & 816 & - & 20 & - \\
\hline 01104455 & Base flow & 20050726 & 1500 & - & - & .19 & .6 & 6.9 \\
\hline 01104455 & Stormflow & 20050814 & 1555 & 20050814 & 1848 & - & 70 & - \\
\hline 01104475 & Base flow & 20041215 & 1455 & - & - & 1.2 & 1.3 & 12.3 \\
\hline 01104475 & Stormflow & 20050708 & 1027 & 20050709 & 851 & - & 10 & - \\
\hline 01104475 & Base flow & 20050726 & 1530 & - & - & .13 & .6 & 7.4 \\
\hline 01104475 & Stormflow & 20050915 & 1012 & 20050915 & 1607 & - & 150 & - \\
\hline 422302071083802 & $\begin{array}{l}\text { Untreated in- } \\
\text { take water }\end{array}$ & 20050720 & 1300 & - & - & - & 1.5 & - \\
\hline 422302071083803 & $\begin{array}{l}\text { Finished pro- } \\
\text { duction water }\end{array}$ & 20050720 & 1330 & - & - & - & .2 & - \\
\hline \multicolumn{9}{|l|}{ Blanks } \\
\hline 01104455 & Field blank & 20050726 & 1501 & - & - & - & - & - \\
\hline USGS-MA-LAB & Source water & 20040601 & 1200 & - & - & - & - & - \\
\hline
\end{tabular}


Table 15. Physical properties and concentrations of major inorganic constituents, nutrients, trace metals, suspended sediments, Escherichia coli bacteria, polyaromatic hydrocarbons, and polar pesticides and metabolites for base-flow and stormflow water samples collected in four subbasins and the Fresh Pond intake structure in the Cambridge, Massachusetts, drinking-water source area for water year 2005.-Continued

[Escherichia coli concentrations were mathematically estimated from subcomposites. PXXXXX, National Water Quality Laboratory parameter code; ${ }^{\circ} \mathrm{C}$, degree Celsius; e, estimated; NTRU, nephelometric turbidity ratio units; USGS-MA-LAB, U.S. Geological Survey-Massachusetts Water Science Center laboratory; <, concentration is less than value shown; id, insufficient data to estimate composite value; - , no data]

\begin{tabular}{|c|c|c|c|c|c|c|c|c|}
\hline $\begin{array}{c}\text { USGS station } \\
\text { number }\end{array}$ & $\begin{array}{l}\text { pH, water, } \\
\text { unfiltered, } \\
\text { field } \\
\text { (standard } \\
\text { units) } \\
\text { P00400 }\end{array}$ & $\begin{array}{l}\text { pH, water, } \\
\text { unfiltered, } \\
\text { laboratory } \\
\text { (standard } \\
\text { units) } \\
\text { P00403 }\end{array}$ & $\begin{array}{c}\text { Specific } \\
\text { conductance, } \\
\text { water, } \\
\text { unfiltered, } \\
\text { laboratory } \\
\text { (microsiemens } \\
\text { per centimeter } \\
\text { at } 25^{\circ} \mathrm{C} \text { ) } \\
\mathrm{P} 90095\end{array}$ & $\begin{array}{c}\text { Specific } \\
\text { conduc- } \\
\text { tance, } \\
\text { water, } \\
\text { unfiltered } \\
\text { (microsie- } \\
\text { mens per } \\
\text { centimeter } \\
\text { at } 25^{\circ} \mathrm{C} \text { ) } \\
\text { P00095 }\end{array}$ & $\begin{array}{c}\text { Tempera- } \\
\text { ture, water } \\
\left({ }^{\circ} \mathrm{C}\right) \\
\text { P00010 }\end{array}$ & $\begin{array}{c}\text { Calcium, wa- } \\
\text { ter, filtered } \\
\text { (milligrams } \\
\text { per liter) } \\
\text { P00915 }\end{array}$ & $\begin{array}{c}\text { Magnesium, } \\
\text { water, } \\
\text { filtered (mil- } \\
\text { ligrams } \\
\text { per liter) } \\
\text { P00925 }\end{array}$ & $\begin{array}{c}\text { Potassium, } \\
\text { water, filtered } \\
\text { (milligrams } \\
\text { per liter) } \\
\text { P00935 }\end{array}$ \\
\hline 01104415 & 6.1 & 7.2 & 1,530 & 1500 & 3.0 & 47.2 & 6.74 & 4.16 \\
\hline 01104415 & 6.8 & 6.7 & e439 & 450 & - & 10.8 & 1.42 & 1.72 \\
\hline 01104415 & 6.5 & 7.3 & 3160 & 3310 & 18.5 & 88.0 & 12.0 & 7.11 \\
\hline 01104415 & 6.3 & 6.9 & 601 & 614 & - & 16.9 & 1.87 & 3.44 \\
\hline 01104433 & 6.5 & 7.2 & 1,980 & 1,960 & 4.1 & 85.0 & 14.0 & 7.32 \\
\hline 01104433 & 6.5 & - & - & 511 & - & - & - & - \\
\hline 01104433 & 6.8 & 7.0 & 1,870 & 1,920 & 22.6 & 78.4 & 13.5 & 7.16 \\
\hline 01104433 & 7.0 & 7.0 & 686 & 827 & - & 30.2 & 4.69 & 4.04 \\
\hline 01104455 & 6.6 & 7.2 & 852 & 843 & 8.4 & 34.4 & 6.28 & 2.43 \\
\hline 01104455 & 6.7 & 6.7 & e218 & 241 & - & 8.20 & 1.47 & 0.88 \\
\hline 01104455 & 6.3 & 7.1 & 1,610 & 1660 & 12.7 & 70.4 & 13.5 & 3.35 \\
\hline 01104455 & 6.1 & 6.9 & 155 & 170 & - & 6.69 & 1.03 & 2.23 \\
\hline 01104475 & 7.1 & 7.2 & 233 & 229 & 4.0 & 17.1 & 3.48 & 2.27 \\
\hline 01104475 & 6.6 & 7.2 & - & 221 & - & 12.9 & 2.39 & 2.44 \\
\hline 01104475 & 7.3 & 7.7 & 332 & 326 & 18.6 & 21.3 & 3.32 & 1.94 \\
\hline 01104475 & 7.0 & 7.3 & 144 & 150 & - & 7.69 & 1.35 & 3.63 \\
\hline 422302071083802 & 6.8 & 7.3 & 526 & 540 & - & 24.0 & 4.39 & 2.26 \\
\hline 422302071083803 & 8.6 & 9.2 & 596 & 612 & - & 23.9 & 4.46 & 19.4 \\
\hline \multicolumn{9}{|l|}{ Blanks } \\
\hline 01104455 & - & 7.9 & $<3$ & 3 & - & $<.02$ & $<.008$ & $<.16$ \\
\hline USGS-MA-LAB & 5.8 & 6.0 & $<3$ & 0 & - & $<.01$ & $<.008$ & $<.16$ \\
\hline
\end{tabular}


Table 15. Physical properties and concentrations of major inorganic constituents, nutrients, trace metals, suspended sediments, Escherichia coli bacteria, polyaromatic hydrocarbons, and polar pesticides and metabolites for base-flow and stormflow water samples collected in four subbasins and the Fresh Pond intake structure in the Cambridge, Massachusetts, drinking-water source area for water year 2005.-Continued

[Escherichia coli concentrations were mathematically estimated from subcomposites. PXXXXX, National Water Quality Laboratory parameter code; ${ }^{\circ} \mathrm{C}$, degree Celsius; e, estimated; NTRU, nephelometric turbidity ratio units; USGS-MA-LAB, U.S. Geological Survey-Massachusetts Water Science Center laboratory; <, concentration is less than value shown; id, insufficient data to estimate composite value; - , no data]

\begin{tabular}{|c|c|c|c|c|c|c|c|c|}
\hline $\begin{array}{c}\text { USGS station } \\
\text { number }\end{array}$ & $\begin{array}{c}\text { Sodium, } \\
\text { water, } \\
\text { filtered } \\
\text { (milligrams } \\
\text { per liter) } \\
\text { P00930 }\end{array}$ & $\begin{array}{c}\text { Alkalinity, } \\
\text { water, filtered, } \\
\text { fixed endpoint } \\
\text { (pH 4.5) titra- } \\
\text { tion, laborato- } \\
\text { ry (milligrams } \\
\text { per liter as } \\
\text { calcium } \\
\text { carbonate) } \\
\text { P29801 }\end{array}$ & $\begin{array}{l}\text { Chloride, } \\
\text { water, } \\
\text { filtered } \\
\text { (milligrams } \\
\text { per liter) } \\
\text { P00940 }\end{array}$ & $\begin{array}{c}\text { Sulfate, } \\
\text { water, } \\
\text { filtered } \\
\text { (milligrams } \\
\text { per liter) } \\
\text { P00945 }\end{array}$ & $\begin{array}{c}\text { Total nitro- } \\
\text { gen (nitrate } \\
\text { + nitrite + } \\
\text { ammonia } \\
\text { + organic- } \\
\text { N), water, } \\
\text { unfiltered, } \\
\text { analytically } \\
\text { determined } \\
\text { (milligrams } \\
\text { per liter) } \\
\text { P62855 }\end{array}$ & $\begin{array}{l}\text { Phosphorus, } \\
\text { water, unfiltered } \\
\text { (milligrams } \\
\text { per liter) } \\
\text { P00665 }\end{array}$ & $\begin{array}{c}\text { Escherichia } \\
\text { coli, m-TEC } \\
\text { MF } \\
\text { method, } \\
\text { water } \\
\text { (colonies per } \\
100 \text { millili- } \\
\text { ters) } \\
\text { P31663 }\end{array}$ & $\begin{array}{c}\text { Cadmium, } \\
\text { water, } \\
\text { unfiltered } \\
\text { (micrograms } \\
\text { per liter) } \\
\text { P01027 }\end{array}$ \\
\hline 01104415 & 254 & 47 & 449 & 25.9 & 2.02 & $\mathrm{e} 0.02$ & 30 & 0.14 \\
\hline 01104415 & 59.6 & 13 & 110 & 6.2 & 1.5 & .31 & id & .06 \\
\hline 01104415 & 538 & 47 & 970 & 28.5 & 1.57 & $<.02$ & 190 & .35 \\
\hline 01104415 & 92.7 & 13 & 160 & 13.4 & 2.38 & .5 & 18,000 & .89 \\
\hline 01104433 & 281 & 64 & 553 & 31.8 & 2.08 & e. 02 & 21 & .29 \\
\hline 01104433 & - & - & - & - & 1.22 & .12 & id & .16 \\
\hline 01104433 & 254 & 58 & 542 & 27.6 & 1.58 & .03 & 89 & .09 \\
\hline 01104433 & 88.9 & 28 & 176 & 18.2 & 2.21 & .18 & id & .31 \\
\hline 01104455 & 121 & 44 & 216 & 24.8 & 1.76 & e. 01 & 53 & .10 \\
\hline 01104455 & 27.2 & 11 & 51.7 & 5.8 & 0.93 & .10 & id & .17 \\
\hline 01104455 & 212 & 47 & 469 & 26.8 & 2.37 & $<.02$ & 1,400 & .18 \\
\hline 01104455 & 20.3 & 9 & 33.7 & 9.2 & 2.96 & .45 & 9,000 & .69 \\
\hline 01104475 & 24.3 & 31 & 37.7 & 20.0 & 1.96 & e. 01 & 4 & $<.04$ \\
\hline 01104475 & 18.2 & 24 & 31.3 & 11.4 & 1.28 & .08 & id & .05 \\
\hline 01104475 & 33.9 & 30 & 66.8 & 18.0 & 1.97 & e. 02 & 310 & $<.04$ \\
\hline 01104475 & 14.9 & 15 & 22.8 & 8.0 & 5.13 & .80 & 43,000 & .44 \\
\hline 422302071083802 & 66.4 & 32 & 134 & 12.9 & .74 & $<.02$ & - & $<.04$ \\
\hline 422302071083803 & 69.7 & 38 & 138 & 25.9 & .94 & $<.02$ & - & $<.04$ \\
\hline \multicolumn{9}{|l|}{ Blanks } \\
\hline 01104455 & $<.20$ & $<5$ & $<.20$ & $<.2$ & e.04 & $<.02$ & $<1$ & $<.04$ \\
\hline USGS-MA-LAB & $<.10$ & $<2$ & $<.20$ & $<.2$ & $<.03$ & $<.01$ & - & $<.04$ \\
\hline
\end{tabular}


Table 15. Physical properties and concentrations of major inorganic constituents, nutrients, trace metals, suspended sediments, Escherichia coli bacteria, polyaromatic hydrocarbons, and polar pesticides and metabolites for base-flow and stormflow water samples collected in four subbasins and the Fresh Pond intake structure in the Cambridge, Massachusetts, drinking-water source area for water year 2005.-Continued

[Escherichia coli concentrations were mathematically estimated from subcomposites. PXXXXX, National Water Quality Laboratory parameter code; ${ }^{\circ} \mathrm{C}$, degree Celsius; e, estimated; NTRU, nephelometric turbidity ratio units; USGS-MA-LAB, U.S. Geological Survey-Massachusetts Water Science Center laboratory; <, concentration is less than value shown; id, insufficient data to estimate composite value; - , no data]

\begin{tabular}{|c|c|c|c|c|c|c|c|c|}
\hline $\begin{array}{c}\text { USGS station } \\
\text { number }\end{array}$ & $\begin{array}{c}\text { Chromium, } \\
\text { water, } \\
\text { unfiltered, } \\
\text { recoverable } \\
\text { (micrograms } \\
\text { per liter) } \\
\text { P01034 }\end{array}$ & $\begin{array}{c}\text { Copper, } \\
\text { water, } \\
\text { unfiltered, } \\
\text { recoverable } \\
\text { (micrograms } \\
\text { per liter) } \\
\text { P01042 }\end{array}$ & $\begin{array}{l}\text { Iron, water, } \\
\text { unfiltered, } \\
\text { recoverable } \\
\text { (micrograms } \\
\text { per liter) } \\
\text { P01045 }\end{array}$ & $\begin{array}{c}\text { Lead, } \\
\text { water, } \\
\text { unfiltered, } \\
\text { recover- } \\
\text { able (mi- } \\
\text { crograms } \\
\text { per liter) } \\
\text { P01051 }\end{array}$ & $\begin{array}{c}\text { Manga- } \\
\text { nese, water, } \\
\text { unfiltered, } \\
\text { recoverable } \\
\text { (micrograms } \\
\text { per liter) } \\
\text { P01055 }\end{array}$ & $\begin{array}{l}\text { Nickel, water, } \\
\text { unfiltered, } \\
\text { recoverable } \\
\text { (micrograms } \\
\text { per liter) } \\
\text { P01067 }\end{array}$ & $\begin{array}{l}\text { Zinc, water, } \\
\text { unfiltered, } \\
\text { recoverable } \\
\text { (micrograms } \\
\text { per liter) } \\
\text { P01092 }\end{array}$ & $\begin{array}{c}2,4,5-T \text {, sur- } \\
\text { rogate, Sched- } \\
\text { ule } 9060 / 2060 \text {, } \\
\text { water, filtered } \\
\text { (percent } \\
\text { recovery) } \\
\text { P99958 }\end{array}$ \\
\hline 01104415 & 2.9 & 3.4 & 300 & 0.45 & 216 & 2.05 & 18 & 99.4 \\
\hline 01104415 & 1.3 & 2.9 & 170 & 1.12 & 96 & .85 & 12 & 84 \\
\hline 01104415 & $<.8$ & 1.6 & 130 & .33 & 332 & .97 & 23 & e87.7 \\
\hline 01104415 & 12.5 & 28.0 & 11,500 & 86.2 & 1,260 & 9.99 & 151 & e168 \\
\hline 01104433 & $<.8$ & 6.2 & 430 & .50 & 283 & 4.05 & 58 & 95.6 \\
\hline 01104433 & 3.3 & 8.0 & 1,340 & 9.14 & 146 & 2.08 & 50 & e153 \\
\hline 01104433 & $<.8$ & 4.2 & 530 & .70 & 406 & 4.24 & 34 & e87.1 \\
\hline 01104433 & 7.5 & 18.4 & 2,740 & 15.0 & 176 & 4.65 & 120 & - \\
\hline 01104455 & $<.8$ & 3.0 & 100 & .27 & 153 & 2.45 & 19 & 89.1 \\
\hline 01104455 & 13.6 & 32.5 & 1,640 & 17.5 & 148 & 3.21 & 70 & e118 \\
\hline 01104455 & $<.8$ & 2.2 & 170 & .64 & 487 & 4.51 & 16 & e93.0 \\
\hline 01104455 & 28.8 & 82.8 & 6,060 & 95.3 & 343 & 9.82 & 284 & e172 \\
\hline 01104475 & $<.8$ & 1.9 & 160 & .44 & 21 & .87 & 4 & 94.5 \\
\hline 01104475 & .9 & 3.0 & 940 & 4.19 & 93 & 1.46 & 8 & 108 \\
\hline 01104475 & $<.8$ & .8 & 80 & .29 & 11 & .91 & e1 & e79.9 \\
\hline 01104475 & 6.7 & 22.8 & 7,350 & 42.6 & 1,120 & 7.87 & 71 & e175 \\
\hline 422302071083802 & $<.8$ & 2.8 & 90 & .28 & 44 & .68 & 3 & e123 \\
\hline 422302071083803 & $<.8$ & $<.6$ & e6 & $<.06$ & 2 & .58 & $<2$ & e127 \\
\hline \multicolumn{9}{|l|}{ Blanks } \\
\hline 01104455 & $<.8$ & $<.6$ & e3 & $<.06$ & 1 & e.09 & $\mathrm{e} 2$ & e80.1 \\
\hline USGS-MA-LAB & $<.8$ & $<.6$ & $<9.0$ & $<.06$ & $<.2$ & $<.16$ & $<2$ & 67.7 \\
\hline
\end{tabular}


Table 15. Physical properties and concentrations of major inorganic constituents, nutrients, trace metals, suspended sediments, Escherichia coli bacteria, polyaromatic hydrocarbons, and polar pesticides and metabolites for base-flow and stormflow water samples collected in four subbasins and the Fresh Pond intake structure in the Cambridge, Massachusetts, drinking-water source area for water year 2005.-Continued

[Escherichia coli concentrations were mathematically estimated from subcomposites. PXXXXX, National Water Quality Laboratory parameter code; ${ }^{\circ} \mathrm{C}$, degree Celsius; e, estimated; NTRU, nephelometric turbidity ratio units; USGS-MA-LAB, U.S. Geological Survey-Massachusetts Water Science Center laboratory; <, concentration is less than value shown; id, insufficient data to estimate composite value; - , no data]

\begin{tabular}{|c|c|c|c|c|c|c|c|c|}
\hline $\begin{array}{l}\text { USGS station } \\
\text { number }\end{array}$ & $\begin{array}{c}\text { 2,4-D methyl } \\
\text { ester, water, } \\
\text { filtered, } \\
\text { recoverable } \\
\text { (micrograms } \\
\text { per liter) } \\
\text { P50470 }\end{array}$ & $\begin{array}{c}\text { 2,4-D, water, } \\
\text { filtered, } \\
\text { recoverable } \\
\text { (micrograms } \\
\text { per liter) } \\
\text { P39732 }\end{array}$ & $\begin{array}{c}\text { 2,4-DB, water, } \\
\text { filtered } \\
\text { (0.7-micron } \\
\text { glass- } \\
\text { fiber filter), } \\
\text { recoverable } \\
\text { (micrograms } \\
\text { per liter) } \\
\text { P38746 }\end{array}$ & $\begin{array}{c}\text { 2-Chloro-4- } \\
\text { isopropyl- } \\
\text { amino6-amino- } \\
\text { s-triazine, } \\
\text { water, } \\
\text { filtered, re- } \\
\text { coverable } \\
\text { (micro- } \\
\text { grams per } \\
\text { liter) } \\
\text { P04040 }\end{array}$ & $\begin{array}{c}\text { 2-Chloro-6- } \\
\text { ethylamino-4- } \\
\text { amino- } \\
\text { s-triazine, wa- } \\
\text { ter, filtered, } \\
\text { recoverable } \\
\text { (micrograms } \\
\text { per liter) } \\
\text { P04038 }\end{array}$ & $\begin{array}{c}\text { 2-Hydroxy-4- } \\
\text { isopropyl- } \\
\text { amino-6-ethyl- } \\
\text { amino-s-triazine, } \\
\text { water, filtered, } \\
\text { recoverable } \\
\text { (micrograms } \\
\text { per liter) } \\
\text { P50355 }\end{array}$ & $\begin{array}{c}\text { 3-Hydroxy } \\
\text { carbofu- } \\
\text { ran, water, } \\
\text { filtered } \\
\text { (0.7-micron } \\
\text { glass- fiber } \\
\text { filter), } \\
\text { recoverable } \\
\text { (micrograms } \\
\text { per liter) } \\
\text { P49308 }\end{array}$ & $\begin{array}{c}\text { Ketocarbo- } \\
\text { furan, water, } \\
\text { filtered, } \\
\text { recoverable } \\
\text { (micrograms } \\
\text { per liter) } \\
\text { P50295 }\end{array}$ \\
\hline 01104415 & $<0.016$ & $\mathrm{e} 0.02$ & $<0.02$ & $<0.03$ & $<0.08$ & $<0.032$ & $<0.008$ & $<0.02$ \\
\hline 01104415 & $<.016$ & $<.04$ & $<.02$ & $<.03$ & $<.08$ & $<.032$ & $<.008$ & - \\
\hline 01104415 & $<.016$ & $<.04$ & $<.02$ & $<.03$ & $<.08$ & $<.032$ & $<.008$ & $<.02$ \\
\hline 01104415 & $<.214$ & e7.92 & $<.02$ & $<.03$ & $<.08$ & $<.032$ & $<.008$ & $<.02$ \\
\hline 01104433 & $<.016$ & $<.04$ & $<.02$ & $<.03$ & $<.08$ & $<.032$ & $<.008$ & $<.02$ \\
\hline 01104433 & $<.016$ & $<.04$ & $<.02$ & $<.03$ & $<.08$ & $<.032$ & $<.008$ & - \\
\hline 01104433 & $<.016$ & $<.04$ & $<.02$ & $<.03$ & $<.08$ & $<.032$ & $<.008$ & $<.02$ \\
\hline 01104433 & $<.016$ & e. 22 & $<.02$ & $<.03$ & $<.08$ & $<.032$ & $<.008$ & $<.02$ \\
\hline 01104455 & $<.016$ & $<.04$ & $<.02$ & $<.03$ & e.01 & $<.032$ & $<.008$ & $<.02$ \\
\hline 01104455 & $<.016$ & $<.04$ & $<.02$ & $<.03$ & $<.08$ & $<.032$ & $<.008$ & - \\
\hline 01104455 & $<.016$ & $<.04$ & $<.02$ & $<.03$ & $<.08$ & $<.032$ & $<.008$ & $<.02$ \\
\hline 01104455 & $<.016$ & e.04 & $<.02$ & $<.03$ & $<.08$ & $<.032$ & $<.008$ & e.08 \\
\hline 01104475 & $<.016$ & $<.04$ & $<.02$ & $<.03$ & $<.08$ & $<.032$ & $<.008$ & $<.02$ \\
\hline 01104475 & $<.016$ & $<.04$ & $<.02$ & $<.03$ & $<.08$ & $<.032$ & $<.008$ & - \\
\hline 01104475 & $<.016$ & $<.04$ & $<.02$ & $<.03$ & $<.08$ & $<.032$ & $<.008$ & $<.02$ \\
\hline 01104475 & $<.139$ & $\mathrm{e} 3.41$ & $<.02$ & $<.03$ & $<.08$ & $<.032$ & $<.008$ & $<.02$ \\
\hline 422302071083802 & $<.016$ & $<.04$ & $<.02$ & $<.03$ & $<.08$ & $<.032$ & $<.008$ & $<.25$ \\
\hline 422302071083803 & $<.016$ & $<.04$ & $<.02$ & $<.03$ & $<.08$ & $<.032$ & $<.008$ & $<.25$ \\
\hline \multicolumn{9}{|l|}{ Blanks } \\
\hline 01104455 & $<.016$ & $<.04$ & $<.02$ & $<.03$ & $<.08$ & $<.032$ & $<.008$ & $<.02$ \\
\hline USGS-MA-LAB & $<.009$ & $<.02$ & $<.02$ & $<.03$ & $<.04$ & $<.008$ & $<.006$ & $<1.50$ \\
\hline
\end{tabular}


Table 15. Physical properties and concentrations of major inorganic constituents, nutrients, trace metals, suspended sediments, Escherichia coli bacteria, polyaromatic hydrocarbons, and polar pesticides and metabolites for base-flow and stormflow water samples collected in four subbasins and the Fresh Pond intake structure in the Cambridge, Massachusetts, drinking-water source area for water year 2005. - Continued

[Escherichia coli concentrations were mathematically estimated from subcomposites. PXXXXX, National Water Quality Laboratory parameter code; ${ }^{\circ} \mathrm{C}$, degree Celsius; e, estimated; NTRU, nephelometric turbidity ratio units; USGS-MA-LAB, U.S. Geological Survey-Massachusetts Water Science Center laboratory; <, concentration is less than value shown; id, insufficient data to estimate composite value; - , no data]

\begin{tabular}{|c|c|c|c|c|c|c|c|c|}
\hline $\begin{array}{c}\text { USGS station } \\
\text { number }\end{array}$ & $\begin{array}{l}\text { 9H-Fluorene, } \\
\text { water, unfil- } \\
\text { tered, recover- } \\
\text { able (micro- } \\
\text { grams per liter) } \\
\text { P34381 }\end{array}$ & $\begin{array}{c}\text { Acenaph- } \\
\text { thene, } \\
\text { water, } \\
\text { unfiltered, } \\
\text { recoverable } \\
\text { (micrograms } \\
\text { per liter) } \\
\text { P34205 }\end{array}$ & $\begin{array}{c}\text { Acenaph- } \\
\text { thylene, } \\
\text { water, } \\
\text { unfiltered, } \\
\text { recoverable } \\
\text { (micrograms } \\
\text { per liter) } \\
\text { P34200 }\end{array}$ & $\begin{array}{c}\text { Acifluorfen, } \\
\text { water, filtered } \\
\text { (0.7-micron } \\
\text { glass-fiber } \\
\text { filter), } \\
\text { recoverable } \\
\text { (micrograms } \\
\text { per liter) } \\
\text { P49315 }\end{array}$ & $\begin{array}{l}\text { Aldicarb } \\
\text { sulfone, } \\
\text { water, } \\
\text { filtered } \\
\text { (0.7-micron } \\
\text { glass-fiber } \\
\text { filter), } \\
\text { recoverable } \\
\text { (micro- } \\
\text { grams per } \\
\text { liter) } \\
\text { P49313 }\end{array}$ & $\begin{array}{c}\text { Aldicarb sulf- } \\
\text { oxide, water, } \\
\text { filtered(0.7-micron } \\
\text { glass-fiber filter), } \\
\text { recoverable } \\
\text { (micrograms per } \\
\text { liter) } \\
\text { P49314 }\end{array}$ & $\begin{array}{c}\text { Aldicarb, } \\
\text { water, } \\
\text { filtered } \\
\text { (0.7-micron } \\
\text { glass- } \\
\text { fiber filter), } \\
\text { recoverable } \\
\text { (micrograms } \\
\text { per liter) } \\
\text { P49312 }\end{array}$ & $\begin{array}{c}\text { Anthracene, } \\
\text { water, } \\
\text { unfiltered, } \\
\text { recoverable } \\
\text { (micrograms } \\
\text { per liter) } \\
\text { P34220 }\end{array}$ \\
\hline 01104415 & $\mathrm{e} 0.005$ & $<2.0$ & $<2.0$ & $<0.028$ & $<0.02$ & $<0.022$ & $<0.04$ & $\mathrm{e} 0.007$ \\
\hline 01104415 & e.03 & e. 02 & $<2$ & $<.028$ & $<.02$ & $<.022$ & $<.04$ & e.05 \\
\hline 01104415 & $<2$ & $<2$ & $<2$ & $<.028$ & $<.02$ & $<.022$ & $<.04$ & $<2$ \\
\hline 01104415 & $<2$ & $<2$ & e. 2 & $<.028$ & $<.02$ & $<.022$ & $<.04$ & e. 2 \\
\hline 01104433 & $<2$ & e. 004 & $<2$ & $<.028$ & $<.02$ & $<.022$ & $<.04$ & e.01 \\
\hline 01104433 & e.02 & $<2$ & $<2$ & $<.028$ & $<.02$ & $<.022$ & $<.04$ & $<2$ \\
\hline 01104433 & $<2$ & $<2$ & $<2$ & $<.028$ & $<.02$ & $<.022$ & $<.04$ & $<2$ \\
\hline 01104433 & e.02 & e. 02 & e.02 & $<.028$ & $<.02$ & $<.022$ & $<.04$ & e. 05 \\
\hline 01104455 & $<2$ & $<2$ & $<2$ & $<.028$ & $<.02$ & $<.022$ & $<.04$ & $<2$ \\
\hline 01104455 & e. 2 & e. 1 & e.03 & $<.028$ & $<.02$ & $<.022$ & $<.04$ & e. 4 \\
\hline 01104455 & $<2$ & $<2$ & $<2$ & $<.028$ & $<.02$ & $<.022$ & $<.04$ & $<2$ \\
\hline 01104455 & e. 3 & e. 2 & $<2$ & $<.028$ & $<.02$ & $<.022$ & $<.04$ & e. 6 \\
\hline 01104475 & $<2$ & $<2$ & $<2$ & $<.028$ & $<.02$ & $<.022$ & $<.04$ & $<2$ \\
\hline 01104475 & e.03 & e.02 & $<2$ & $<.028$ & $<.02$ & $<.022$ & $<.04$ & e.05 \\
\hline 01104475 & $<2$ & $<2$ & $<2$ & $<.028$ & $<.02$ & $<.022$ & $<.04$ & $<2$ \\
\hline 01104475 & e. 1 & e.07 & e.09 & $<.028$ & $<.02$ & $<.022$ & $<.04$ & e. 3 \\
\hline 422302071083802 & $<2$ & $<2$ & $<2$ & $<.028$ & $<.02$ & $<.022$ & $<.04$ & $<2$ \\
\hline 422302071083803 & $<2$ & $<2$ & $<2$ & $<.028$ & $<.02$ & $<.022$ & $<.04$ & $<2$ \\
\hline \multicolumn{9}{|l|}{ Blanks } \\
\hline 01104455 & $<2$ & $<2$ & $<2$ & $<.028$ & $<.02$ & $<.022$ & $<.04$ & $<2$ \\
\hline USGS-MA-LAB & $<2$ & $<2$ & $<2$ & $<.007$ & $<.02$ & $<.008$ & $<.04$ & $<2$ \\
\hline
\end{tabular}


Table 15. Physical properties and concentrations of major inorganic constituents, nutrients, trace metals, suspended sediments, Escherichia coli bacteria, polyaromatic hydrocarbons, and polar pesticides and metabolites for base-flow and stormflow water samples collected in four subbasins and the Fresh Pond intake structure in the Cambridge, Massachusetts, drinking-water source area for water year 2005. - Continued

[Escherichia coli concentrations were mathematically estimated from subcomposites. PXXXXX, National Water Quality Laboratory parameter code; ${ }^{\circ} \mathrm{C}$, degree Celsius; e, estimated; NTRU, nephelometric turbidity ratio units; USGS-MA-LAB, U.S. Geological Survey-Massachusetts Water Science Center laboratory; <, concentration is less than value shown; id, insufficient data to estimate composite value; - , no data]

\begin{tabular}{|c|c|c|c|c|c|c|c|c|}
\hline $\begin{array}{l}\text { USGS station } \\
\text { number }\end{array}$ & $\begin{array}{c}\text { Atrazine, } \\
\text { water, } \\
\text { filtered, } \\
\text { recoverable } \\
\text { (micrograms } \\
\text { per liter) } \\
\text { P39632 }\end{array}$ & $\begin{array}{c}\text { Barban, } \\
\text { surrogate, } \\
\text { Schedules } \\
2060 / 9060, \\
\text { water, } \\
\text { filtered } \\
\text { (percent } \\
\text { recovery) } \\
\text { P90640 }\end{array}$ & $\begin{array}{c}\text { Bendio- } \\
\text { carb, water, } \\
\text { filtered, } \\
\text { recoverable } \\
\text { (micrograms } \\
\text { per liter) } \\
\text { P50299 }\end{array}$ & $\begin{array}{l}\text { Benomyl, } \\
\text { water, } \\
\text { filtered, re- } \\
\text { coverable } \\
\text { (micro- } \\
\text { grams per } \\
\text { liter) } \\
\text { P50300 }\end{array}$ & $\begin{array}{l}\text { Bensulfu- } \\
\text { ron, water, } \\
\text { filtered, } \\
\text { recoverable } \\
\text { (micro- } \\
\text { grams } \\
\text { per liter) } \\
\text { P61693 }\end{array}$ & $\begin{array}{c}\text { Bentazon, } \\
\text { water, filtered } \\
\text { (0.7-micron } \\
\text { glass- } \\
\text { fiber filter), } \\
\text { recoverable } \\
\text { (micrograms } \\
\text { per liter) } \\
\text { P38711 }\end{array}$ & $\begin{array}{c}\text { Benzo[a] } \\
\text { anthracene, } \\
\text { water, } \\
\text { unfiltered, } \\
\text { recoverable } \\
\text { (micrograms } \\
\text { per liter) } \\
\text { P34526 }\end{array}$ & $\begin{array}{c}\text { Benzo[a] } \\
\text { pyrene, } \\
\text { water, } \\
\text { unfiltered, } \\
\text { recoverable } \\
\text { (micrograms } \\
\text { per liter) } \\
\text { P34247 }\end{array}$ \\
\hline 01104415 & $<0.008$ & e92.0 & $<0.02$ & $<0.022$ & $<0.02$ & $<0.01$ & $<2.0$ & $<1.0$ \\
\hline 01104415 & $<.008$ & e33.9 & $<.02$ & $<.022$ & $<.02$ & $<.01$ & e. 4 & e. 6 \\
\hline 01104415 & $<.008$ & $\mathrm{e} 44.4$ & $<.02$ & $<.022$ & $<.02$ & $<.01$ & $<2$ & $<1$ \\
\hline 01104415 & $<.008$ & $\mathrm{e} 42.6$ & $<.02$ & $<.022$ & $<.02$ & $<.01$ & e. 8 & e1 \\
\hline 01104433 & $<.008$ & e76 & $<.02$ & $<.022$ & $<.02$ & $<.01$ & $<2$ & $<1$ \\
\hline 01104433 & $<.008$ & e18 & $<.02$ & $<.022$ & $<.02$ & $<.01$ & e. 1 & e. 2 \\
\hline 01104433 & $<.008$ & e15.6 & $<.02$ & $<.022$ & $<.02$ & $<.01$ & $<2$ & $<1$ \\
\hline 01104433 & $<.008$ & 66.2 & $<.02$ & $<.022$ & $<.02$ & $<.01$ & e. 3 & e. 4 \\
\hline 01104455 & $<.008$ & e83.8 & $<.02$ & $<.022$ & $<.02$ & $<.01$ & $<2$ & $<1$ \\
\hline 01104455 & $<.008$ & e16.6 & $<.02$ & $<.022$ & $<.02$ & $<.01$ & e2 & e2 \\
\hline 01104455 & $<.008$ & 90.7 & $<.02$ & $<.022$ & $<.02$ & $<.01$ & $<2$ & $<1$ \\
\hline 01104455 & $<.008$ & e389 & $<.02$ & $<.022$ & $<.02$ & $<.01$ & e2 & 3 \\
\hline 01104475 & $<.008$ & e108 & $<.02$ & .03 & $<.02$ & $<.01$ & $<2$ & $<1$ \\
\hline 01104475 & $<.008$ & $\mathrm{e} 23.5$ & $<.02$ & e. 176 & $<.02$ & $<.01$ & e. 2 & e. 3 \\
\hline 01104475 & $<.008$ & 81.9 & $<.02$ & e. 159 & $<.02$ & $<.01$ & $<2$ & $<1$ \\
\hline 01104475 & $<.008$ & 89.7 & $<.02$ & $<.022$ & $<.02$ & $<.01$ & $\mathrm{e} 1$ & 2 \\
\hline 422302071083802 & $<.008$ & e39 & $<.02$ & $<.022$ & $<.02$ & $<.01$ & $<2$ & $<1$ \\
\hline 422302071083803 & $<.008$ & 118 & $<.02$ & $<.022$ & $<.02$ & $<.01$ & $<2$ & $<1$ \\
\hline \multicolumn{9}{|l|}{ Blanks } \\
\hline 01104455 & $<.008$ & 92.2 & $<.02$ & $<.022$ & $<.02$ & $<.01$ & $<2$ & $<1$ \\
\hline USGS-MA-LAB & $<.009$ & 84.9 & $<.03$ & $<.004$ & $<.02$ & $<.01$ & $<2$ & $<1$ \\
\hline
\end{tabular}


Table 15. Physical properties and concentrations of major inorganic constituents, nutrients, trace metals, suspended sediments, Escherichia coli bacteria, polyaromatic hydrocarbons, and polar pesticides and metabolites for base-flow and stormflow water samples collected in four subbasins and the Fresh Pond intake structure in the Cambridge, Massachusetts, drinking-water source area for water year 2005.-Continued

[Escherichia coli concentrations were mathematically estimated from subcomposites. PXXXXX, National Water Quality Laboratory parameter code; ${ }^{\circ} \mathrm{C}$, degree Celsius; e, estimated; NTRU, nephelometric turbidity ratio units; USGS-MA-LAB, U.S. Geological Survey-Massachusetts Water Science Center laboratory; <, concentration is less than value shown; id, insufficient data to estimate composite value; - , no data]

\begin{tabular}{|c|c|c|c|c|c|c|c|c|}
\hline $\begin{array}{l}\text { USGS station } \\
\text { number }\end{array}$ & $\begin{array}{l}\text { Benzo }[b] \text { fluor- } \\
\text { anthene, water, } \\
\text { unfiltered, } \\
\text { recoverable } \\
\text { (micrograms per } \\
\text { liter) } \\
\text { P34230 }\end{array}$ & $\begin{array}{c}\text { Benzo[ghi] } \\
\text { perylene, } \\
\text { water, } \\
\text { unfiltered, } \\
\text { recoverable } \\
\text { (micrograms } \\
\text { per liter) } \\
\text { P34521 }\end{array}$ & $\begin{array}{c}\text { Benzo[k] } \\
\text { fluoran- } \\
\text { thene, water, } \\
\text { unfiltered, } \\
\text { recoverable } \\
\text { (micrograms } \\
\text { per liter) } \\
\text { P34242 }\end{array}$ & $\begin{array}{l}\text { Bromacil, } \\
\text { water, } \\
\text { filtered, re- } \\
\text { coverable } \\
\text { (micro- } \\
\text { grams per } \\
\text { liter) } \\
\text { P04029 }\end{array}$ & $\begin{array}{c}\text { Bromoxynil, } \\
\text { water, } \\
\text { filtered } \\
\text { (0.7-micron } \\
\text { glass-fiber } \\
\text { filter), } \\
\text { recoverable } \\
\text { (micro- } \\
\text { grams per } \\
\text { liter) } \\
\text { P49311 }\end{array}$ & $\begin{array}{l}\text { Caffeine, } \\
\text { water, filtered, } \\
\text { recoverable } \\
\text { (micrograms } \\
\text { per liter) } \\
\text { P50305 }\end{array}$ & $\begin{array}{c}\text { Caffeine-13C, } \\
\text { surrogate, } \\
\text { schedule } \\
\text { 9060/2060, } \\
\text { water, } \\
\text { filtered } \\
\text { (percent } \\
\text { recovery) } \\
\text { P99959 }\end{array}$ & $\begin{array}{c}\text { Carbaryl, water, } \\
\text { filtered } \\
\text { (0.7-micron } \\
\text { glass- fiber } \\
\text { filter), } \\
\text { recoverable } \\
\text { (micrograms } \\
\text { per liter) } \\
\text { P49310 }\end{array}$ \\
\hline 01104415 & $<2.0$ & $<3.0$ & $<2.0$ & $<0.02$ & $<0.03$ & 0.02 & 112.0 & $<0.02$ \\
\hline 01104415 & e1 & e. 6 & e. 3 & $<.02$ & $<.03$ & .77 & 83.9 & .03 \\
\hline 01104415 & $<2$ & $<3$ & $<2$ & $<.02$ & $<.03$ & $<.018$ & e118 & $<.02$ \\
\hline 01104415 & $\mathrm{e} 2$ & e. 5 & e. 6 & $<.02$ & $<.03$ & e. 773 & e124 & e.03 \\
\hline 01104433 & $<2$ & $<3$ & $<2$ & $<.02$ & $<.03$ & .48 & 91.6 & $<.02$ \\
\hline 01104433 & e. 4 & e. 2 & e. 1 & $<.02$ & $<.03$ & .26 & 98.9 & $<.02$ \\
\hline 01104433 & $<2$ & $<3$ & $<2$ & $<.02$ & $<.03$ & e. 678 & e117 & $<.02$ \\
\hline 01104433 & e. 7 & e. 3 & e. 3 & $<.02$ & $<.03$ & e1.69 & 97.5 & e.01 \\
\hline 01104455 & $<2$ & $<3$ & $<2$ & $<.02$ & $<.03$ & .18 & 104 & $<.02$ \\
\hline 01104455 & e1 & $\mathrm{e} 2$ & $\mathrm{e} 1$ & $<.02$ & $<.03$ & .23 & 97.7 & $<.02$ \\
\hline 01104455 & $<2$ & $<3$ & $<2$ & $<.02$ & $<.03$ & $<.018$ & e117 & $<.02$ \\
\hline 01104455 & 5 & $\mathrm{e} 1$ & $\mathrm{e} 2$ & $<.02$ & $<.03$ & e. 583 & e360 & $<.02$ \\
\hline 01104475 & $<2$ & $<3$ & $<2$ & $<.02$ & $<.03$ & e. 009 & 102 & $<.02$ \\
\hline 01104475 & e. 4 & e. 3 & e. 1 & $<.02$ & $<.03$ & .04 & 88.2 & $<.02$ \\
\hline 01104475 & $<2$ & $<3$ & $<2$ & $<.02$ & $<.03$ & $<.018$ & e105 & $<.02$ \\
\hline 01104475 & 3 & e2 & $\mathrm{e} 1$ & $<.02$ & $<.03$ & .23 & 100 & e.01 \\
\hline 422302071083802 & $<2$ & $<3$ & $<2$ & $<.02$ & $<.03$ & .02 & 98.6 & $<.02$ \\
\hline 422302071083803 & $<2$ & $<3$ & $<2$ & $<.02$ & $<.03$ & $<.018$ & 116 & $<.02$ \\
\hline \multicolumn{9}{|l|}{ Blanks } \\
\hline 01104455 & $<2$ & $<3$ & $<2$ & $<.02$ & $<.03$ & $<.018$ & e154 & $<.02$ \\
\hline USGS-MA-LAB & $<2$ & $<3$ & $<2$ & $<.03$ & $<.02$ & $<.010$ & 127 & $<.03$ \\
\hline
\end{tabular}


Table 15. Physical properties and concentrations of major inorganic constituents, nutrients, trace metals, suspended sediments, Escherichia coli bacteria, polyaromatic hydrocarbons, and polar pesticides and metabolites for base-flow and stormflow water samples collected in four subbasins and the Fresh Pond intake structure in the Cambridge, Massachusetts, drinking-water source area for water year 2005.-Continued

[Escherichia coli concentrations were mathematically estimated from subcomposites. PXXXXX, National Water Quality Laboratory parameter code; ${ }^{\circ} \mathrm{C}$, degree Celsius; e, estimated; NTRU, nephelometric turbidity ratio units; USGS-MA-LAB, U.S. Geological Survey-Massachusetts Water Science Center laboratory; <, concentration is less than value shown; id, insufficient data to estimate composite value; - , no data]

\begin{tabular}{|c|c|c|c|c|c|c|c|c|}
\hline $\begin{array}{l}\text { USGS station } \\
\text { number }\end{array}$ & $\begin{array}{c}\text { Carbofuran, } \\
\text { water, filtered } \\
\text { (0.7-micron } \\
\text { glass- } \\
\text { fiber filter), } \\
\text { recoverable } \\
\text { (micrograms per } \\
\text { liter) } \\
\text { P49309 }\end{array}$ & $\begin{array}{c}\text { Chloramben } \\
\text { methyles- } \\
\text { ter, water, } \\
\text { filtered, } \\
\text { recoverable } \\
\text { (micrograms } \\
\text { per liter) } \\
\text { P61188 }\end{array}$ & $\begin{array}{l}\text { Chlorimuron, } \\
\text { water, } \\
\text { filtered, } \\
\text { recoverable } \\
\text { (micrograms } \\
\text { per liter) } \\
\text { P50306 }\end{array}$ & $\begin{array}{l}\text { Chlorodi- } \\
\text { amino-s- } \\
\text { triazine, } \\
\text { water, } \\
\text { filtered, re- } \\
\text { coverable } \\
\text { (micro- } \\
\text { grams per } \\
\text { liter) } \\
\text { P04039 }\end{array}$ & $\begin{array}{c}\text { Chlorotha- } \\
\text { Ionil, water, } \\
\text { filtered } \\
\text { (0.7-micron } \\
\text { glass- fi- } \\
\text { ber filter), } \\
\text { recoverable } \\
\text { (micro- } \\
\text { grams per } \\
\text { liter) } \\
\text { P49306 }\end{array}$ & $\begin{array}{l}\text { Chrysene, wa- } \\
\text { ter, unfiltered, } \\
\text { recoverable } \\
\text { (micrograms } \\
\text { per liter) } \\
\text { P34320 }\end{array}$ & $\begin{array}{c}\text { Clopy- } \\
\text { ralid, water, } \\
\text { filtered } \\
\text { (0.7-micron } \\
\text { glass-fi- } \\
\text { ber filter), } \\
\text { recoverable } \\
\text { (micrograms } \\
\text { per liter) } \\
\text { P49305 }\end{array}$ & $\begin{array}{c}\text { Cycloate, wa- } \\
\text { ter, filtered, } \\
\text { recoverable } \\
\text { (micrograms } \\
\text { per liter) } \\
\text { P04031 }\end{array}$ \\
\hline 01104415 & $<0.016$ & $<0.02$ & $<0.032$ & $<0.04$ & $<0.04$ & $<3.0$ & $<0.02$ & $<0.01$ \\
\hline 01104415 & $<.016$ & $<.02$ & $<.032$ & $<.04$ & $<.04$ & e.7 & $<.02$ & $<.01$ \\
\hline 01104415 & $<.016$ & $<.02$ & $<.032$ & $<.04$ & $<.04$ & $<3$ & $<.02$ & $<.01$ \\
\hline 01104415 & $<.016$ & $<.02$ & $<.032$ & $<.04$ & $<.04$ & e. 9 & $<.02$ & $<.01$ \\
\hline 01104433 & $<.016$ & $<.02$ & $<.032$ & $<.04$ & $<.04$ & $<3$ & $<.02$ & $<.01$ \\
\hline 01104433 & $<.016$ & $<.02$ & $<.032$ & $<.04$ & $<.04$ & e. 3 & $<.02$ & $<.01$ \\
\hline 01104433 & $<.016$ & $<.02$ & $<.032$ & $<.04$ & $<.04$ & $<3$ & $<.02$ & $<.01$ \\
\hline 01104433 & $<.016$ & $<.02$ & $<.032$ & $<.04$ & $<.04$ & e. 5 & $<.02$ & $<.01$ \\
\hline 01104455 & $<.016$ & $<.02$ & $<.032$ & $<.04$ & $<.04$ & $<3$ & $<.02$ & $<.01$ \\
\hline 01104455 & $<.016$ & $<.02$ & $<.032$ & $<.04$ & $<.04$ & e2 & $<.02$ & $<.01$ \\
\hline 01104455 & $<.016$ & $<.02$ & $<.032$ & $<.04$ & $<.04$ & $<3$ & $<.02$ & $<.01$ \\
\hline 01104455 & $<.016$ & $<.02$ & $<.032$ & $<.04$ & $<.04$ & 4 & $<.02$ & $<.01$ \\
\hline 01104475 & $<.016$ & $<.02$ & $<.032$ & $<.04$ & $<.04$ & $<3$ & $<.02$ & $<.01$ \\
\hline 01104475 & $<.016$ & $<.02$ & $<.032$ & $<.04$ & $<5.54$ & e. 3 & $<.02$ & $<.01$ \\
\hline 01104475 & $<.016$ & $<.02$ & $<.032$ & $<.04$ & $<.04$ & $<3$ & $<.02$ & $<.01$ \\
\hline 01104475 & $<.016$ & $<.02$ & $<.032$ & $<.04$ & $<.04$ & e2 & $<.02$ & $<.01$ \\
\hline 422302071083802 & $<.016$ & $<.02$ & $<.032$ & $<.04$ & $<.04$ & $<3$ & $<.02$ & $<.01$ \\
\hline 422302071083803 & $<.016$ & $<.02$ & $<.032$ & $<.04$ & $<.04$ & $<3$ & $<.02$ & $<.01$ \\
\hline \multicolumn{9}{|l|}{ Blanks } \\
\hline 01104455 & $<.016$ & $<.02$ & $<.032$ & $<.04$ & $<.04$ & $<3$ & $<.02$ & $<.01$ \\
\hline USGS-MA-LAB & $<.006$ & $<.02$ & $<.010$ & $<.01$ & $<.04$ & $<3$ & $<.01$ & $<.01$ \\
\hline
\end{tabular}


Table 15. Physical properties and concentrations of major inorganic constituents, nutrients, trace metals, suspended sediments, Escherichia coli bacteria, polyaromatic hydrocarbons, and polar pesticides and metabolites for base-flow and stormflow water samples collected in four subbasins and the Fresh Pond intake structure in the Cambridge, Massachusetts, drinking-water source area for water year 2005.-Continued

[Escherichia coli concentrations were mathematically estimated from subcomposites. PXXXXX, National Water Quality Laboratory parameter code; ${ }^{\circ} \mathrm{C}$, degree Celsius; e, estimated; NTRU, nephelometric turbidity ratio units; USGS-MA-LAB, U.S. Geological Survey-Massachusetts Water Science Center laboratory; <, concentration is less than value shown; id, insufficient data to estimate composite value; - , no data]

\begin{tabular}{|c|c|c|c|c|c|c|c|c|}
\hline $\begin{array}{c}\text { USGS station } \\
\text { number }\end{array}$ & $\begin{array}{c}\text { Dacthal mono- } \\
\text { acid, water, } \\
\text { filtered (0.7-mi- } \\
\text { cron glass- fiber } \\
\text { filter), recover- } \\
\text { able (micro- } \\
\text { grams per liter) } \\
\text { P49304 }\end{array}$ & $\begin{array}{c}\text { Dibenzo[a,h] } \\
\text { anthracene, } \\
\text { water, } \\
\text { unfiltered, } \\
\text { recoverable } \\
\text { (micrograms } \\
\text { per liter) } \\
\text { P34556 }\end{array}$ & $\begin{array}{c}\text { Dicamba, } \\
\text { water, filtered } \\
\text { (0.7-micron } \\
\text { glass-fiber } \\
\text { filter), } \\
\text { recoverable } \\
\text { (micrograms } \\
\text { per liter) } \\
\text { P38442 }\end{array}$ & $\begin{array}{c}\text { Dichlor- } \\
\text { prop, water, } \\
\text { filtered } \\
\text { (0.7-micron } \\
\text { glass- } \\
\text { fiber filter), } \\
\text { recover- } \\
\text { able (mi- } \\
\text { crograms } \\
\text { per liter) } \\
\text { P49302 }\end{array}$ & $\begin{array}{c}\text { Dinoseb, } \\
\text { water, } \\
\text { filtered } \\
\text { (0.7-micron } \\
\text { glass- } \\
\text { fiber filter), } \\
\text { recoverable } \\
\text { (micro- } \\
\text { grams } \\
\text { per liter) } \\
\text { P49301 }\end{array}$ & $\begin{array}{c}\text { Diphenamid, } \\
\text { water, filtered, } \\
\text { recoverable } \\
\text { (micrograms } \\
\text { per liter) } \\
\text { P04033 }\end{array}$ & $\begin{array}{c}\text { Diuron, wa- } \\
\text { ter, filtered } \\
\text { (0.7-micron } \\
\text { glass-fiber } \\
\text { filter), } \\
\text { recoverable } \\
\text { (micrograms } \\
\text { per liter) } \\
\text { P49300 }\end{array}$ & $\begin{array}{c}\text { Fenuron, wa- } \\
\text { ter, filtered } \\
\text { (0.7-micron } \\
\text { glass-fiber } \\
\text { filter), } \\
\text { recoverable } \\
\text { (micrograms } \\
\text { per liter) } \\
\text { P49297 }\end{array}$ \\
\hline 01104415 & $<0.03$ & $<3.0$ & $<0.04$ & $<0.03$ & $<0.04$ & $<0.01$ & $<0.01$ & $<0.02$ \\
\hline 01104415 & $<.03$ & $<3$ & $<.04$ & $<.03$ & $<.04$ & $<.01$ & $<.01$ & $<.02$ \\
\hline 01104415 & $<.03$ & $<3$ & $<.04$ & $<.03$ & $<.04$ & $<.01$ & .02 & $<.02$ \\
\hline 01104415 & $<.03$ & $<3$ & $<.09$ & $<.03$ & $<.04$ & $<.01$ & $<.01$ & $<.02$ \\
\hline 01104433 & $<.03$ & $<3$ & $<.04$ & $<.03$ & $<.04$ & $<.01$ & .08 & $<.02$ \\
\hline 01104433 & $<.03$ & $<3$ & $<.04$ & $<.03$ & $<.04$ & $<.01$ & $<.01$ & $<.02$ \\
\hline 01104433 & $<.03$ & $<3$ & $<.04$ & $<.03$ & $<.04$ & $<.01$ & e. 03 & $<.02$ \\
\hline 01104433 & $<.03$ & e. 09 & $<.04$ & $<.03$ & $<.04$ & $<.01$ & e. 14 & $<.02$ \\
\hline 01104455 & $<.03$ & $<3$ & $<.04$ & $<.03$ & $<.04$ & $<.01$ & $<.01$ & $<.02$ \\
\hline 01104455 & $<.03$ & e. 5 & $<.04$ & $<.03$ & $<.04$ & $<.01$ & $<.01$ & $<.02$ \\
\hline 01104455 & $<.03$ & $<3$ & $<.04$ & $<.03$ & $<.04$ & $<.01$ & $<.01$ & $<.02$ \\
\hline 01104455 & $<.03$ & e. 4 & $<.04$ & $<.03$ & $<.04$ & $<.01$ & $<.01$ & $<.02$ \\
\hline 01104475 & $<.03$ & $<3$ & $<.04$ & $<.03$ & $<.04$ & $<.01$ & $<.01$ & $<.02$ \\
\hline 01104475 & $<.03$ & e. 08 & $<.04$ & $<.03$ & $<.04$ & $<.01$ & $<.01$ & $<.02$ \\
\hline 01104475 & $<.03$ & $<3$ & $<.04$ & $<.03$ & $<.04$ & $<.01$ & $<.01$ & $<.02$ \\
\hline 01104475 & $<.03$ & e. 4 & $<.04$ & $<.03$ & $<.04$ & $<.01$ & $<.01$ & $<.02$ \\
\hline 422302071083802 & $<.03$ & $<3$ & $<.04$ & $<.03$ & $<.04$ & $<.01$ & $<.01$ & $<.02$ \\
\hline 422302071083803 & $<.03$ & $<3$ & $<.04$ & $<.03$ & $<.04$ & $<.01$ & $<.01$ & $<.02$ \\
\hline \multicolumn{9}{|l|}{ Blanks } \\
\hline 01104455 & $<.03$ & $<3$ & $<.04$ & $<.03$ & $<.04$ & $<.01$ & $<.01$ & $<.02$ \\
\hline USGS-MA-LAB & $<.01$ & $<3$ & $<.01$ & $<.01$ & $<.01$ & $<.03$ & $<.01$ & $<.03$ \\
\hline
\end{tabular}


Table 15. Physical properties and concentrations of major inorganic constituents, nutrients, trace metals, suspended sediments, Escherichia coli bacteria, polyaromatic hydrocarbons, and polar pesticides and metabolites for base-flow and stormflow water samples collected in four subbasins and the Fresh Pond intake structure in the Cambridge, Massachusetts, drinking-water source area for water year 2005.-Continued

[Escherichia coli concentrations were mathematically estimated from subcomposites. PXXXXX, National Water Quality Laboratory parameter code; ${ }^{\circ} \mathrm{C}$, degree Celsius; e, estimated; NTRU, nephelometric turbidity ratio units; USGS-MA-LAB, U.S. Geological Survey-Massachusetts Water Science Center laboratory; <, concentration is less than value shown; id, insufficient data to estimate composite value; - , no data]

\begin{tabular}{|c|c|c|c|c|c|c|c|c|}
\hline $\begin{array}{l}\text { USGS station } \\
\text { number }\end{array}$ & $\begin{array}{l}\text { Flumetsulam, } \\
\text { water, } \\
\text { filtered, } \\
\text { recoverable } \\
\text { (micrograms } \\
\text { per liter) } \\
\text { P61694 }\end{array}$ & $\begin{array}{c}\text { Fluometuron, } \\
\text { water, filtered } \\
\text { (0.7-micron } \\
\text { glass-fiber } \\
\text { filter) recover- } \\
\text { able (micro- } \\
\text { grams } \\
\text { per liter) } \\
\text { P38811 }\end{array}$ & $\begin{array}{c}\text { Fluoran- } \\
\text { thene, water, } \\
\text { unfiltered, } \\
\text { recoverable } \\
\text { (micrograms } \\
\text { per liter) } \\
\text { P34376 }\end{array}$ & $\begin{array}{l}\text { Imazaquin, } \\
\text { water, } \\
\text { filtered, re- } \\
\text { coverable } \\
\text { (micro- } \\
\text { grams per } \\
\text { liter) } \\
\text { P50356 }\end{array}$ & $\begin{array}{l}\text { Imazetha- } \\
\text { pyr, water, } \\
\text { filtered, } \\
\text { recoverable } \\
\text { (micro- } \\
\text { grams } \\
\text { per liter) } \\
\text { P50407 }\end{array}$ & $\begin{array}{l}\text { Imidacloprid, } \\
\text { water, filtered, } \\
\text { recoverable } \\
\text { (micrograms } \\
\text { per liter) } \\
\text { P61695 }\end{array}$ & $\begin{array}{c}\text { Indeno[1,2,3- } \\
\text { cd] pyrene, } \\
\text { water, } \\
\text { unfiltered, } \\
\text { recoverable } \\
\text { (micrograms } \\
\text { per liter) } \\
\text { P34403 }\end{array}$ & $\begin{array}{c}\text { Linuron, } \\
\text { water, filtered } \\
\text { (0.7-micron } \\
\text { glass-fi- } \\
\text { ber filter), } \\
\text { recoverable } \\
\text { (micrograms } \\
\text { per liter) } \\
\text { P38478 }\end{array}$ \\
\hline 01104415 & $<0.04$ & $<0.02$ & $\mathrm{e} 0.01$ & $<0.04$ & $<0.04$ & $\mathrm{e} 0.011$ & $<3.0$ & $<0.01$ \\
\hline 01104415 & $<.04$ & $<.02$ & e1 & $<.04$ & $<.04$ & .18 & e. 6 & $<.01$ \\
\hline 01104415 & $<.04$ & $<.02$ & $<2$ & $<.04$ & $<.04$ & $<.020$ & $<3$ & $<.01$ \\
\hline 01104415 & $<.04$ & $<.02$ & e2 & $<.04$ & $<.04$ & e. 375 & e. 5 & $<.01$ \\
\hline 01104433 & $<.04$ & $<.02$ & e. 05 & $<.04$ & $<.04$ & $<.020$ & $<3$ & $<.01$ \\
\hline 01104433 & $<.04$ & $<.02$ & e. 5 & $<.04$ & $<.04$ & .23 & e. 2 & $<.01$ \\
\hline 01104433 & $<.04$ & $<.02$ & e. 1 & e.01 & $<.04$ & e. 067 & $<3$ & $<.01$ \\
\hline 01104433 & $<.04$ & $<.02$ & e. 9 & e.06 & $<.04$ & $<.020$ & e. 3 & $<.01$ \\
\hline 01104455 & $<.04$ & $<.02$ & $<2$ & $<.04$ & $<.04$ & $<.020$ & $<3$ & $<.01$ \\
\hline 01104455 & $<.04$ & $<.02$ & 5 & $<.04$ & $<.04$ & $<.020$ & e1 & $<.01$ \\
\hline 01104455 & $<.04$ & $<.02$ & e.04 & e.01 & $<.04$ & $<.020$ & $<3$ & $<.01$ \\
\hline 01104455 & $<.04$ & $<.02$ & 9 & $<.04$ & $<.04$ & e. 113 & e1 & $<.01$ \\
\hline 01104475 & $<.04$ & $<.02$ & e.02 & $<.04$ & $<.04$ & .52 & $<3$ & $<.01$ \\
\hline 01104475 & $<.04$ & $<.02$ & e.7 & $<.04$ & $<.04$ & $\mathrm{e} 1.00$ & e. 2 & $<.01$ \\
\hline 01104475 & $<.04$ & $<.02$ & $<2$ & $<.04$ & $<.04$ & .36 & $<3$ & $<.01$ \\
\hline 01104475 & $<.04$ & $<.02$ & 4 & $<.19$ & $<.04$ & .34 & $\mathrm{e} 1$ & $<.01$ \\
\hline 422302071083802 & $<.04$ & $<.02$ & e.01 & $<.04$ & $<.04$ & .02 & $<3$ & $<.01$ \\
\hline 422302071083803 & $<.04$ & $<.02$ & $<2$ & $<.04$ & $<.04$ & $<.020$ & $<3$ & $<.01$ \\
\hline \multicolumn{9}{|l|}{ Blanks } \\
\hline 01104455 & $<.04$ & $<.02$ & $<2$ & $<.04$ & $<.04$ & $<.020$ & $<3$ & $<.01$ \\
\hline USGS-MA-LAB & $<.01$ & $<.03$ & $<2$ & $<.02$ & $<.02$ & $<.007$ & $<3$ & $<.01$ \\
\hline
\end{tabular}


Table 15. Physical properties and concentrations of major inorganic constituents, nutrients, trace metals, suspended sediments, Escherichia coli bacteria, polyaromatic hydrocarbons, and polar pesticides and metabolites for base-flow and stormflow water samples collected in four subbasins and the Fresh Pond intake structure in the Cambridge, Massachusetts, drinking-water source area for water year 2005. - Continued

[Escherichia coli concentrations were mathematically estimated from subcomposites. PXXXXX, National Water Quality Laboratory parameter code; ${ }^{\circ} \mathrm{C}$, degree Celsius; e, estimated; NTRU, nephelometric turbidity ratio units; USGS-MA-LAB, U.S. Geological Survey-Massachusetts Water Science Center laboratory; <, concentration is less than value shown; id, insufficient data to estimate composite value; - , no data]

\begin{tabular}{|c|c|c|c|c|c|c|c|c|}
\hline $\begin{array}{l}\text { USGS station } \\
\text { number }\end{array}$ & $\begin{array}{c}\text { MCPA, } \\
\text { water, filtered } \\
\text { (0.7-micron } \\
\text { glass-fiber } \\
\text { filter), recover- } \\
\text { able (micro- } \\
\text { grams per liter) } \\
\text { P38482 }\end{array}$ & $\begin{array}{c}\text { MCPB, wa- } \\
\text { ter, filtered } \\
\text { (0.7-micron } \\
\text { glass-fiber } \\
\text { filter), } \\
\text { recoverable } \\
\text { (micrograms } \\
\text { per liter) } \\
\text { P38487 }\end{array}$ & $\begin{array}{c}\text { Metal- } \\
\text { axyl, water, } \\
\text { filtered, } \\
\text { recoverable } \\
\text { (micrograms } \\
\text { per liter) } \\
\text { P50359 }\end{array}$ & $\begin{array}{c}\text { Methio- } \\
\text { carb, water, } \\
\text { filtered } \\
\text { (0.7-micron } \\
\text { glass- } \\
\text { fiber filter), } \\
\text { recover- } \\
\text { able (mi- } \\
\text { crograms } \\
\text { per liter) } \\
\text { P38501 }\end{array}$ & $\begin{array}{l}\text { Methomyl, } \\
\text { water, } \\
\text { filtered } \\
\text { (0.7-micron } \\
\text { glass- } \\
\text { fiber filter), } \\
\text { recoverable } \\
\text { (micro- } \\
\text { grams per } \\
\text { liter) } \\
\text { P49296 }\end{array}$ & $\begin{array}{l}\text { Metsulfuron, } \\
\text { water, filtered, } \\
\text { recoverable } \\
\text { (micrograms } \\
\text { per liter) } \\
\text { P61697 }\end{array}$ & $\begin{array}{c}\text { N-(4-Chlo- } \\
\text { rophenyl)- } \\
\text { N'-methy- } \\
\text { lurea, water, } \\
\text { filtered, re- } \\
\text { coverable } \\
\text { (micrograms } \\
\text { per liter) } \\
\text { P61692 }\end{array}$ & $\begin{array}{c}\text { Neburon, } \\
\text { water, filtered } \\
\text { (0.7-micron } \\
\text { glass-fi- } \\
\text { ber filter), } \\
\text { recoverable } \\
\text { (micrograms } \\
\text { per liter) } \\
\text { P49294 }\end{array}$ \\
\hline 01104415 & - & $<0.01$ & $<0.01$ & $<0.010$ & $<0.020$ & $<0.03$ & $<0.04$ & $<0.01$ \\
\hline 01104415 & $<0.03$ & $<.01$ & $<.01$ & $<.010$ & $<.020$ & $<.30$ & $<.04$ & $<.01$ \\
\hline 01104415 & $<.03$ & $<.01$ & $<.01$ & $<.010$ & - & $<.03$ & $<.04$ & $<.01$ \\
\hline 01104415 & $<.05$ & $<.01$ & $<.01$ & $<.010$ & $<.020$ & $\mathrm{e} 1.40$ & $<.04$ & $<.01$ \\
\hline 01104433 & - & $<.01$ & $<.01$ & $<.010$ & $<.020$ & $<.03$ & $<.04$ & $<.01$ \\
\hline 01104433 & $<.06$ & $<.01$ & $<.01$ & $<.010$ & $<.020$ & $\mathrm{e} 1.26$ & $<.04$ & $<.01$ \\
\hline 01104433 & $<.03$ & $<.01$ & $<.01$ & $<.010$ & - & e3.90 & $<.04$ & $<.01$ \\
\hline 01104433 & $<.03$ & $<.01$ & $<.01$ & $<.010$ & $<.020$ & $<.03$ & $<.04$ & $<.01$ \\
\hline 01104455 & - & $<.01$ & $<.01$ & $<.010$ & $<.020$ & $<.03$ & $<.04$ & $<.01$ \\
\hline 01104455 & $<.03$ & $<.01$ & $<.01$ & $<.010$ & $<.020$ & $<.41$ & $<.04$ & $<.01$ \\
\hline 01104455 & $<.03$ & $<.01$ & $<.01$ & $<.010$ & - & $<.03$ & $<.04$ & $<.01$ \\
\hline 01104455 & $<.09$ & $<.01$ & $<.01$ & $<.010$ & $<.020$ & $\mathrm{e} 2.52$ & $<.04$ & $<.01$ \\
\hline 01104475 & - & $<.01$ & .04 & $<.010$ & $<.020$ & $<.03$ & $<.04$ & $<.01$ \\
\hline 01104475 & $<.03$ & $<.01$ & .02 & $<.010$ & $<.020$ & $<.03$ & $<.04$ & $<.01$ \\
\hline 01104475 & $<.03$ & $<.01$ & e.01 & $<.010$ & - & $<.03$ & $<.04$ & $<.01$ \\
\hline 01104475 & e. 16 & $<.01$ & $<.01$ & $<.010$ & $<.020$ & $<.03$ & $<.04$ & $<.01$ \\
\hline 422302071083802 & $<.03$ & $<.01$ & $<.01$ & $<.010$ & $<.020$ & $<.03$ & $<.04$ & $<.01$ \\
\hline 422302071083803 & $<.03$ & $<.01$ & $<.01$ & $<.010$ & $<.020$ & $<.03$ & $<.04$ & $<.01$ \\
\hline \multicolumn{9}{|l|}{ Blanks } \\
\hline 01104455 & $<.03$ & $<.01$ & $<.01$ & $<.010$ & - & $<.03$ & $<.04$ & $<.01$ \\
\hline USGS-MA-LAB & $<.02$ & $<.01$ & $<.02$ & $<.008$ & $<.004$ & $<.03$ & $<.02$ & $<.01$ \\
\hline
\end{tabular}


Table 15. Physical properties and concentrations of major inorganic constituents, nutrients, trace metals, suspended sediments, Escherichia coli bacteria, polyaromatic hydrocarbons, and polar pesticides and metabolites for base-flow and stormflow water samples collected in four subbasins and the Fresh Pond intake structure in the Cambridge, Massachusetts, drinking-water source area for water year 2005.-Continued

[Escherichia coli concentrations were mathematically estimated from subcomposites. PXXXXX, National Water Quality Laboratory parameter code; ${ }^{\circ} \mathrm{C}$, degree Celsius; e, estimated; NTRU, nephelometric turbidity ratio units; USGS-MA-LAB, U.S. Geological Survey-Massachusetts Water Science Center laboratory; <, concentration is less than value shown; id, insufficient data to estimate composite value; - , no data]

\begin{tabular}{|c|c|c|c|c|c|c|c|c|}
\hline $\begin{array}{l}\text { USGS station } \\
\text { number }\end{array}$ & $\begin{array}{l}\text { Nicosulfuron, } \\
\text { water, filtered, } \\
\text { recoverable } \\
\text { (micrograms per } \\
\text { liter) } \\
\text { P50364 }\end{array}$ & $\begin{array}{c}\text { Nitroben- } \\
\text { zene, water, } \\
\text { unfiltered, } \\
\text { recoverable } \\
\text { (micrograms } \\
\text { per liter) } \\
\text { P34447 }\end{array}$ & $\begin{array}{c}\text { Norflura- } \\
\text { zon, water, } \\
\text { filtered } \\
\text { (0.7-micron } \\
\text { glass- } \\
\text { fiber filter), } \\
\text { recoverable } \\
\text { (micrograms } \\
\text { per liter) } \\
\text { P49293 }\end{array}$ & $\begin{array}{c}\text { Oryzalin, } \\
\text { water, } \\
\text { filtered } \\
\text { (0.7-micron } \\
\text { glass- } \\
\text { fiber filter), } \\
\text { recover- } \\
\text { able (mi- } \\
\text { crograms } \\
\text { per liter) } \\
\text { P49292 }\end{array}$ & $\begin{array}{c}\text { Oxamyl, } \\
\text { water, } \\
\text { filtered } \\
\text { (0.7-micron } \\
\text { glass- } \\
\text { fiber filter), } \\
\text { recoverable } \\
\text { (micro- } \\
\text { grams } \\
\text { per liter) } \\
\text { P38866 }\end{array}$ & $\begin{array}{c}\text { Phenan- } \\
\text { threne, water, } \\
\text { unfiltered, } \\
\text { recoverable } \\
\text { (micrograms } \\
\text { per liter) } \\
\text { P34461 }\end{array}$ & $\begin{array}{c}\text { Picloram, } \\
\text { water, } \\
\text { filtered (0.7- } \\
\text { micron } \\
\text { glass- } \\
\text { fiber filter), } \\
\text { recoverable } \\
\text { (micrograms } \\
\text { per liter) } \\
\text { P49291 }\end{array}$ & $\begin{array}{c}\text { Propham, } \\
\text { water, filtered } \\
\text { (0.7-micron } \\
\text { glass- fi- } \\
\text { ber filter), } \\
\text { recoverable } \\
\text { (micrograms } \\
\text { per liter) } \\
\text { P49236 }\end{array}$ \\
\hline 01104415 & $<0.04$ & $<2$ & $<0.02$ & $<0.01$ & $<0.03$ & $<2.0$ & $<0.03$ & $<0.030$ \\
\hline 01104415 & $<.04$ & $<2$ & $<.02$ & $<.01$ & $<.03$ & e.6 & $<.03$ & $<.030$ \\
\hline 01104415 & $<.04$ & $<2$ & $<.02$ & $<.01$ & $<.03$ & $<2$ & $<.03$ & $<.030$ \\
\hline 01104415 & $<.04$ & $<2$ & $<.02$ & $<.01$ & $<.03$ & e. 6 & $<.03$ & $<.030$ \\
\hline 01104433 & $<.04$ & $<2$ & $<.02$ & $<.01$ & $<.03$ & e. 02 & $<.03$ & $<.030$ \\
\hline 01104433 & $<.04$ & $<2$ & $<.02$ & $<.01$ & $<.03$ & e. 2 & $<.03$ & $<.030$ \\
\hline 01104433 & $<.04$ & $<2$ & $<.02$ & $<.01$ & $<.03$ & $<2$ & $<.03$ & $<.030$ \\
\hline 01104433 & $<.04$ & $<2$ & $<.02$ & $<.01$ & $<.03$ & e. 3 & $<.03$ & $<.030$ \\
\hline 01104455 & $<.04$ & $<2$ & .15 & $<.01$ & $<.03$ & $<2$ & $<.03$ & $<.030$ \\
\hline 01104455 & $<.04$ & $<2$ & .12 & $<.01$ & $<.03$ & 2 & $<.03$ & $<.030$ \\
\hline 01104455 & $<.04$ & $<2$ & .08 & $<.01$ & $<.03$ & $<2$ & $<.03$ & $<.030$ \\
\hline 01104455 & $<.04$ & $<2$ & $<.02$ & $<.01$ & $<.03$ & 4 & $<.03$ & $<.030$ \\
\hline 01104475 & $<.04$ & $<2$ & $<.02$ & $<.01$ & $<.03$ & $<2$ & $<.03$ & $<.030$ \\
\hline 01104475 & $<.04$ & $<2$ & $<.02$ & $<.01$ & $<.03$ & e. 3 & $<.03$ & $<.030$ \\
\hline 01104475 & $<.04$ & $<2$ & $<.02$ & $<.01$ & $<.03$ & $<2$ & $<.03$ & $<.030$ \\
\hline 01104475 & $<.04$ & $<2$ & $<.03$ & $<.01$ & $<.03$ & $\mathrm{e} 2$ & $<.03$ & $<.030$ \\
\hline 422302071083802 & $<.04$ & $<2$ & $<.02$ & $<.01$ & $<.03$ & e. 01 & $<.03$ & $<.030$ \\
\hline 422302071083803 & $<.04$ & $<2$ & $<.02$ & $<.01$ & $<.03$ & $<2$ & $<.03$ & $<.030$ \\
\hline \multicolumn{9}{|l|}{ Blanks } \\
\hline 01104455 & $<.04$ & $<2$ & $<.02$ & $<.01$ & $<.03$ & $<2$ & $<.03$ & $<.030$ \\
\hline USGS-MA-LAB & $<.01$ & $<2$ & $<.02$ & - & $<.01$ & $<2$ & $<.02$ & $<.010$ \\
\hline
\end{tabular}


Table 15. Physical properties and concentrations of major inorganic constituents, nutrients, trace metals, suspended sediments, Escherichia coli bacteria, polyaromatic hydrocarbons, and polar pesticides and metabolites for base-flow and stormflow water samples collected in four subbasins and the Fresh Pond intake structure in the Cambridge, Massachusetts, drinking-water source area for water year 2005. - Continued

[Escherichia coli concentrations were mathematically estimated from subcomposites. PXXXXX, National Water Quality Laboratory parameter code; ${ }^{\circ} \mathrm{C}$, degree Celsius; e, estimated; NTRU, nephelometric turbidity ratio units; USGS-MA-LAB, U.S. Geological Survey-Massachusetts Water Science Center laboratory; <, concentration is less than value shown; id, insufficient data to estimate composite value; - , no data]

\begin{tabular}{|c|c|c|c|c|c|c|c|c|}
\hline $\begin{array}{l}\text { USGS station } \\
\text { number }\end{array}$ & $\begin{array}{l}\text { Propicon- } \\
\text { azole, water, } \\
\text { filtered, } \\
\text { recoverable } \\
\text { (micrograms } \\
\text { per liter) } \\
\text { P50471 }\end{array}$ & $\begin{array}{c}\text { Propoxur, } \\
\text { water, } \\
\text { filtered } \\
\text { (0.7-micron } \\
\text { glass- } \\
\text { fiber filter), } \\
\text { recoverable } \\
\text { (micrograms } \\
\text { per liter) } \\
\text { P38538 }\end{array}$ & $\begin{array}{c}\text { Pyrene, } \\
\text { water, } \\
\text { unfiltered, } \\
\text { recoverable } \\
\text { (micrograms } \\
\text { per liter) } \\
\text { P34469 }\end{array}$ & $\begin{array}{c}\text { Siduron, } \\
\text { water, } \\
\text { filtered, re- } \\
\text { coverable } \\
\text { (micro- } \\
\text { grams per } \\
\text { liter) } \\
\text { P38548 }\end{array}$ & $\begin{array}{c}\text { Sulfome- } \\
\text { turon, wa- } \\
\text { ter, filtered, } \\
\text { recoverable } \\
\text { (micro- } \\
\text { grams } \\
\text { per liter) } \\
\text { P50337 }\end{array}$ & $\begin{array}{c}\text { Tebuthiuron, } \\
\text { water, filtered } \\
\text { (0.7-micron } \\
\text { glass-fiber } \\
\text { filter), recover- } \\
\text { able (micro- } \\
\text { grams per } \\
\text { liter) } \\
\text { P82670 }\end{array}$ & $\begin{array}{l}\text { Terbacil, wa- } \\
\text { ter, filtered, } \\
\text { recoverable } \\
\text { (micrograms } \\
\text { per liter) } \\
\text { P04032 }\end{array}$ & $\begin{array}{c}\text { Triclopyr, wa- } \\
\text { ter, filtered } \\
\text { (0.7-micron } \\
\text { glass-fiber } \\
\text { filter), } \\
\text { recoverable } \\
\text { (micrograms } \\
\text { per liter) } \\
\text { P49235 }\end{array}$ \\
\hline 01104415 & $<0.01$ & $<0.008$ & e0.009 & $<0.02$ & $<0.038$ & $<0.026$ & $<0.016$ & $<0.03$ \\
\hline 01104415 & $<.01$ & $<.008$ & e1 & e.01 & $<.038$ & $<.026$ & $<.016$ & $<.03$ \\
\hline 01104415 & $<.01$ & $<.008$ & $<2$ & $<.02$ & $<.038$ & $<.026$ & $<.016$ & $<.03$ \\
\hline 01104415 & $<.01$ & $<.008$ & $\mathrm{e} 2$ & e.06 & $<.038$ & $<.026$ & $<.016$ & $\mathrm{e} 1.44$ \\
\hline 01104433 & $<.01$ & $<.008$ & e.03 & $<.02$ & $<.038$ & $<.026$ & $<.016$ & $<.03$ \\
\hline 01104433 & $<.01$ & e. 246 & e. 4 & e.01 & $<.038$ & $<.026$ & $<.016$ & $<.03$ \\
\hline 01104433 & $<.01$ & $<.008$ & e.09 & $<.02$ & $<.038$ & $<.026$ & $<.016$ & $<.03$ \\
\hline 01104433 & $<.01$ & $<.008$ & e. 6 & e.04 & $<.038$ & $<.026$ & $<.016$ & $<.03$ \\
\hline 01104455 & $<.01$ & $<.008$ & $<2$ & $<.02$ & $<.038$ & $<.026$ & $<.016$ & $<.03$ \\
\hline 01104455 & $<.01$ & $<.008$ & 3 & e.01 & $<.038$ & $<.026$ & $<.016$ & $<.03$ \\
\hline 01104455 & $<.01$ & $<.008$ & $<2$ & $<.02$ & $<.038$ & $<.026$ & $<.016$ & $<.03$ \\
\hline 01104455 & $<.01$ & $<.008$ & 7 & e.03 & $<.038$ & $<.026$ & $<.016$ & $<.03$ \\
\hline 01104475 & .18 & $<.008$ & e.01 & $<.02$ & $<.038$ & $<.026$ & $<.016$ & $<.03$ \\
\hline 01104475 & .54 & $<.008$ & e.5 & e.01 & $<.038$ & $<.026$ & $<.016$ & .25 \\
\hline 01104475 & .12 & $<.008$ & $<2$ & $<.02$ & $<.038$ & $<.026$ & $<.016$ & $<.03$ \\
\hline 01104475 & $<.01$ & $<.008$ & 3 & $<.02$ & $<.038$ & $<.026$ & $<.016$ & $<.03$ \\
\hline 422302071083802 & $<.01$ & $<.008$ & e.005 & e.004 & $<.038$ & $<.026$ & $<.016$ & $<.03$ \\
\hline 422302071083803 & $<.01$ & $<.008$ & $<2$ & $<.02$ & $<.038$ & $<.026$ & $<.016$ & $<.03$ \\
\hline \multicolumn{9}{|l|}{ Blanks } \\
\hline 01104455 & $<.01$ & $<.008$ & $<2$ & $<.02$ & $<.038$ & $<.026$ & $<.016$ & $<.03$ \\
\hline USGS-MA-LAB & $<.02$ & $<.008$ & $<2$ & $<.02$ & $<.009$ & $<.006$ & $<.010$ & $<.02$ \\
\hline
\end{tabular}


Table 15. Physical properties and concentrations of major inorganic constituents, nutrients, trace metals, suspended sediments, Escherichia coli bacteria, polyaromatic hydrocarbons, and polar pesticides and metabolites for base-flow and stormflow water samples collected in four subbasins and the Fresh Pond intake structure in the Cambridge, Massachusetts, drinking-water source area for water year 2005. - Continued

[Escherichia coli concentrations were mathematically estimated from subcomposites. PXXXXX, National Water Quality Laboratory parameter code; ${ }^{\circ} \mathrm{C}$, degree Celsius; e, estimated; NTRU, nephelometric turbidity ratio units; USGS-MA-LAB, U.S. Geological Survey-Massachusetts Water Science Center laboratory; <, concentration is less than value shown; id, insufficient data to estimate composite value; - , no data]

\begin{tabular}{|c|c|c|c|c|c|c|c|}
\hline $\begin{array}{l}\text { USGS station } \\
\text { number }\end{array}$ & $\begin{array}{l}\text { Naphtha- } \\
\text { lene, water, } \\
\text { unfiltered, } \\
\text { recoverable } \\
\text { (micrograms } \\
\text { per liter) } \\
\text { P34696 }\end{array}$ & $\begin{array}{c}\text { 2-Fluoro- } \\
\text { biphenyl, } \\
\text { surrogate } \\
\text { (percent } \\
\text { recovery) } \\
\text { P49279 }\end{array}$ & $\begin{array}{l}\text { Terphenyl- } \\
\text { d14, surro- } \\
\text { gate (percent } \\
\text { recovery) } \\
\text { P49278 }\end{array}$ & $\begin{array}{l}\text { Suspended } \\
\text { sediment, } \\
\text { sieve } \\
\text { diameter } \\
\text { (percent } \\
\text { smaller } \\
\text { than } 0.063 \\
\text { millime- } \\
\text { ters) } \\
\text { P70331 }\end{array}$ & $\begin{array}{l}\text { Suspended } \\
\text { sediment, } \\
\text { sieve } \\
\text { diameter } \\
\text { (percent } \\
\text { smaller } \\
\text { than } 0.25 \\
\text { millimeters) } \\
\text { P70333 }\end{array}$ & $\begin{array}{c}\text { Suspended } \\
\text { sediment } \\
\text { concentration } \\
\text { (milligrams } \\
\text { per liter) } \\
\text { P80154 }\end{array}$ & $\begin{array}{c}\text { Perylene, } \\
\text { water, } \\
\text { unfiltered, } \\
\text { recoverable } \\
\text { (micrograms } \\
\text { per liter) } \\
\text { P77801 }\end{array}$ \\
\hline 01104415 & $<2.0$ & 97 & 98 & 64 & 91 & 3 & - \\
\hline 01104415 & e. 03 & 93 & 60 & 46 & 73 & 144 & $\mathrm{e} 0.1$ \\
\hline 01104415 & $<2$ & 87 & 69 & 56 & 78 & 2 & $<1$ \\
\hline 01104415 & $<2$ & 100 & 55 & 11 & 19 & 1040 & e. 2 \\
\hline 01104433 & $<2$ & 95 & 77 & 67 & 87 & 2 & - \\
\hline 01104433 & e. 03 & 95 & 60 & 82 & 93 & 29 & e. 05 \\
\hline 01104433 & $<2$ & 91 & 76 & 68 & 99 & 17 & $<1$ \\
\hline 01104433 & $<2$ & 100 & 54 & 85 & 99 & 59 & e. 08 \\
\hline 01104455 & $<2$ & 95 & 97 & 50 & 79 & 2 & - \\
\hline 01104455 & e. 04 & 91.2 & 54.6 & 67 & 93 & 54 & e. 4 \\
\hline 01104455 & $<2$ & 93 & 89 & 55 & 82 & 2 & $<1$ \\
\hline 01104455 & $<2$ & 92 & 47 & 59 & 89 & 248 & e. 6 \\
\hline 01104475 & $<2$ & 50 & 61 & 61 & 87 & 4 & - \\
\hline 01104475 & e. 01 & 99 & 72 & 50 & 80 & 29 & e. 06 \\
\hline 01104475 & $<2$ & 100 & 93 & 71 & 86 & 1 & $<1$ \\
\hline 01104475 & e. 04 & 83 & 42 & 70 & 95 & 348 & e. 4 \\
\hline 422302071083802 & $<2$ & 100 & 95 & - & - & 1 & - \\
\hline 422302071083803 & $<2$ & 98 & 32 & - & - & 1 & - \\
\hline \multicolumn{8}{|l|}{ Blanks } \\
\hline 01104455 & e.07 & 90 & 87 & 0 & 0 & $<1$ & $<1$ \\
\hline USGS-MA-LAB & $<2$ & 95 & 59 & 0 & 0 & $<1$ & - \\
\hline
\end{tabular}


Table 16. Concentrations of Escherichia coli for water samples collected during base flow and storms in four subbasins in the Cambridge, Massachusetts, drinking-water source area for water year 2005.

$[B$, water sample collected during base flow; $\mathrm{S}$, composite water sample collected during stormflow; $\mathrm{PB}$, processing equipment blank; FB, field blank; R, replicate sample; col/100 mL, colony per 100 milliliters; —, no data; <, less than; K, nonideal colony count]

\begin{tabular}{|c|c|c|c|c|c|c|}
\hline $\begin{array}{l}\text { USGS station } \\
\text { identifier }\end{array}$ & $\begin{array}{c}\text { Sample } \\
\text { type }\end{array}$ & $\begin{array}{l}\text { Begin } \\
\text { date }\end{array}$ & $\begin{array}{l}\text { Begin } \\
\text { time }\end{array}$ & $\begin{array}{l}\text { End } \\
\text { date }\end{array}$ & $\begin{array}{l}\text { End } \\
\text { time }\end{array}$ & $\begin{array}{l}\text { Escherichia coli } \\
\text { (col/100 mL) }\end{array}$ \\
\hline 01104415 & $\mathrm{~B}$ & 20041215 & 1025 & - & - & 30 \\
\hline 01104415 & $\mathrm{~S}$ & 20050708 & 1038 & 20050708 & 1935 & 36,000 \\
\hline 01104415 & $\mathrm{~S}$ & 20050708 & 1941 & 20050708 & 2338 & 11,000 \\
\hline 01104415 & B & 20050726 & 1210 & - & - & 190 \\
\hline 01104415 & $\mathrm{~S}$ & 20050814 & 1751 & 20050815 & 1157 & 18,000 \\
\hline 01104433 & $\mathrm{~B}$ & 20041215 & 1205 & - & - & 21 \\
\hline 01104433 & $\mathrm{~S}$ & 20050708 & 1127 & 20050708 & 2119 & 3,600 \\
\hline 01104433 & $\mathrm{~S}$ & 20050709 & 19 & 20050709 & 900 & 4,300 \\
\hline 01104433 & $\mathrm{~B}$ & 20050726 & 1245 & - & - & 89 \\
\hline 01104433 & $\mathrm{~S}$ & 20050915 & 1008 & 20050915 & 1443 & 6,500 \\
\hline 01104433 & $\mathrm{~S}$ & 20050915 & 1457 & 20050915 & 1919 & 5,300 \\
\hline 01104455 & B & 20041215 & 1355 & - & - & 53 \\
\hline 01104455 & $\mathrm{~S}$ & 20050708 & 1006 & 20050708 & 2018 & 9,200 \\
\hline 01104455 & $\mathrm{~S}$ & 20050708 & 2030 & 20050709 & 816 & 3,700 \\
\hline 01104455 & B & 20050726 & 1500 & - & - & 1,400 \\
\hline 01104455 & $\mathrm{~S}$ & 20050814 & 1555 & 20050814 & 1848 & 9,000 \\
\hline 01104475 & $\mathrm{~B}$ & 20041215 & 1455 & - & - & K4 \\
\hline 01104475 & $\mathrm{~S}$ & 20050708 & 1028 & 20050708 & 2031 & 5,700 \\
\hline 01104475 & S & 20050708 & 2043 & 20050709 & 632 & 1,700 \\
\hline 01104475 & B & 20050726 & 1530 & - & - & 310 \\
\hline 01104475 & $\mathrm{~S}$ & 20050915 & 1012 & 20050915 & 1607 & 43,000 \\
\hline \multicolumn{7}{|c|}{ Blanks } \\
\hline 01104415 & PB & 20050726 & 1211 & - & - & $<1$ \\
\hline 01104433 & $\mathrm{~PB}$ & 20040827 & 915 & - & - & $<1$ \\
\hline 01104455 & FB & 20050726 & 1501 & - & - & $<1$ \\
\hline 01104455 & $\mathrm{~PB}$ & 20050814 & 1749 & - & - & $<1$ \\
\hline 01104433 & $\mathrm{~PB}$ & 20050915 & 1530 & - & - & $<1$ \\
\hline \multicolumn{7}{|c|}{ Replicate samples } \\
\hline 01104433 & B & 20041215 & 1205 & - & - & 21 \\
\hline 01104433 & $\mathrm{R}$ & 20041215 & 1205 & - & - & K11 \\
\hline 01104455 & $\mathrm{~B}$ & 20041215 & 1355 & - & - & 53 \\
\hline 01104455 & $\mathrm{R}$ & 20041215 & 1355 & - & - & 84 \\
\hline 01104475 & $\mathrm{~B}$ & 20041215 & 1455 & - & - & K4 \\
\hline 01104475 & $\mathrm{R}$ & 20041215 & 1455 & - & - & K11 \\
\hline 01104415 & $\mathrm{~S}$ & 20050814 & 1751 & 20050815 & 1157 & 18,000 \\
\hline 01104415 & $\mathrm{R}$ & 20050814 & 1752 & - & - & 12,000 \\
\hline 01104433 & $\mathrm{~S}$ & 20050915 & 1008 & 20050915 & 1443 & 6,500 \\
\hline 01104433 & $\mathrm{R}$ & 20050915 & 1009 & - & - & 7,500 \\
\hline
\end{tabular}




\section{Glossary}

Definitions in this glossary are adapted from the U.S. Geological Survey Annual Water Data Report for Massachusetts and Rhode Island (Socolow and others, 2004).

A

alkalinity The capacity of solutes in an aqueous system to neutralize acid. Alkalinity is determined by titration of a filtered sample.

annual runoff The total quantity of water that is discharged (runs off) from a drainage basin in a year. This report presents annual runoff data as volumes in millions of gallons per day per square mile and as depths of water on the drainage basin in inches.

\section{B}

base flow Sustained flow of a stream in the absence of direct runoff. It includes natural and human-induced stream flows. Natural base flow is sustained largely by ground-water discharge.

C

capacity The volume of water in a reservoir or lake. Unless otherwise indicated, volume is computed on the basis of a level pool and does not include bank storage.

control A feature in the channel that physically affects the water-surface elevation and thereby determines the stage-discharge relation at the station. This feature may be a constriction of the channel, a bedrock outcrop, a gravel bar, an artificial structure, or a uniform cross section over a long reach of the channel.

D

datalogger A microprocessor-based dataacquisition system designed specifically to acquire, process, and store data. Most dataloggers are capable of transmitting data by phone modem, cellular modem, radio, or satellitecommunication systems.

datum A surface or point relative to which measurements of height and horizontal position are reported. A vertical datum is a horizontal surface used as the zero point for measurements of stream stage or altitude; a horizontal datum is a reference for positions given in terms of latitude-longitude, state plane coordinates, or Univeral Transverse Mercado coordinates.

discharge or flow The rate at which matter passes through a cross section of a stream channel or other water body per unit of time. The term commonly refers to the volume of water (including, unless otherwise stated, any sediment or other constituents suspended or dissolved in the water) that passes a cross section in a stream channel, canal, or pipeline within a given period of time.

dissolved The material in a representative water sample that passes through a 0.45 -micrometer membrane filter. This is a convenient operational definition used by Federal and State agencies that collect waterquality data. Determinations of dissolved constituent concentrations are made on sample water that has been filtered.

dissolved oxygen Molecular oxygen $\left(\mathrm{O}_{2}\right.$, oxygen gas) dissolved in water. The concentration in water is a function of atmospheric pressure, temperature, and dissolved-solids concentration of the water. The ability of water to retain oxygen decreases with increasing temperature or dissolved-solids concentration. Photosynthesis and respiration by plants commonly cause diurnal variations in dissolved-oxygen concentration in water from some streams.

drainage area The area measured in a horizontal plane upstream from the location from which surface runoff from precipitation normally drains by gravity to the stream at that location. Drainage areas given herein include all closed basins, or noncontributing areas, within the area unless otherwise specified.

drainage basin A part of the Earth's surface that contains a drainage system with a common outlet for its surface runoff. 


\section{E}

Escherichia coli (E. coli) Bacteria present in the intestine and feces of warm-blooded animals. E. coli are a member species of the fecal coliform group of indicator bacteria. In the laboratory, they are defined as those bacteria that produce yellow or yellow-brown colonies on a filter pad saturated with urea substrate broth after primary culturing for 22 to 24 hours at $44.5^{\circ} \mathrm{C}$ on mTEC medium (nutrient medium for bacterial growth). Their concentrations are expressed as number of colonies per $100 \mathrm{~mL}$ of sample.

estimated (E) concentration value Reported when an analyte is detected and all criteria for a positive result are met. If the concentration is less than the method detection limit (MDL), an "E" code will be reported with the value. Also, if the analyte is qualitatively identified as present, but the quantitative determination is substantially more uncertain, the USGS National Water Quality Laboratory will identify the result with an "E" code even though the measured value is greater than the MDL. A value reported with an "E" code should be used with caution. When no analyte is detected in a sample, the default reporting value is the MDL preceded by a less than sign $(<)$.

\section{G}

gill radiation shield A louvered structure which allows air to pass freely through the shield thereby serving to keep a temperature sensor at or near ambient temperature. The shield's white color reflects solar radiation.

\section{I}

inch In this report, this unit is used for the depth to which a drainage area would be covered with water if all of the runoff for a given time period were uniformly distributed over it. See also annual runoff

instantaneous discharge The discharge at a particular instant of time. See also discharge

\section{L}

laboratory reporting level (LRL) The concentration generally equal to twice the yearly determined long-term method detection level (LT-MDL). The LRL controls false negative error. The probability of falsely reporting a nondetection for a sample containing an analyte at a concentration equal to or greater than the LRL is predicted to be less than or equal to 1 percent. The value of the LRL will be reported with a "less than" $(<)$ remark code for samples in which the analyte was not detected. The USGS National Water Quality Laboratory collects quality-control data from selected analytical methods on a continuing basis to determine LT-MDLs and to establish LRLs. These values are reevaluated annually on the basis of the most current quality-control data and, therefore, may change.

\section{long-term method detection level (LT-MDL)}

A concentration derived by determining the standard deviation of a minimum of 24 methoddetection-limit spike-sample measurements over an extended period of time. LT-MDL data are collected on a continuous basis to assess year-to-year variations in the LT-MDL. The LTMDL controls false positive error. The chance of falsely reporting a concentration at or greater than the LT-MDL for a sample not containing the analyte is predicted to be less than or equal to 1 percent.

\section{M}

mean discharge The arithmetic mean of individual daily mean discharges during a specific period. See also discharge

metabolite Any substance produced, used, or remaining during or after metabolism (that is, digestion).

method detection limit The minimum concentration of a substance that can be measured and reported with 99-percent confidence that the analyte concentration is greater than zero. It is determined from the analysis of a sample in a given matrix containing the analyte. At the MDL concentration, the risk of a false positive is predicted to be less than or equal to 1 percent.

micrograms per liter A unit expressing the concentration of chemical constituents in water as mass (micrograms) of constituent per unit volume (liter) of water. One thousand micrograms per liter is equivalent to 1 milligram per liter. One microgram per liter is equivalent to 1 part per billion.

microsiemens per centimeter A unit expressing the amount of electrical conductivity of a solution as measured between opposite faces of a centimeter cube of solution at a specified temperature. Siemens is the International System of Units nomenclature. It is synonymous with mhos and is the reciprocal of resistance in ohms. 
milligrams per liter A unit for expressing the concentration of chemical constituents in water as the mass (milligrams) of constituent per unit volume (liter) of water. The concentration of suspended sediment also is expressed in milligrams per liter and is based on the mass of dry sediment per liter of water-sediment mixture.

million gallons per day The total volume of water represented by flow over a 24-hour period of time. Expressed as a rate, it is the volume of water per unit time (that is, seconds, minutes, or hours) that if held constant, would represent a flow for a 24-hour period. One $\mathrm{Mgal} / \mathrm{d}$ is equivalent to 1.547 cubic feet per second or 0.04381 cubic meters per second.

million gallons per day per square mile The average volume of water in millions of gallons from each square mile of area drained. The runoff is assumed to be distributed uniformly in time and area. See also annual runoff

minimum reporting level (MRL) The smallest measured concentration of a constituent that may be reliably reported by using a given analytical method.

$\mathbf{N}$

National Geodetic Vertical Datum of 1929 (NGVD 29) A fixed reference adopted as a standard geodetic datum for elevations determined by leveling. It formerly was called "Sea Level Datum of 1929" or "mean sea level." Although the datum was derived from the mean sea level at 26 tide stations, it does not necessarily represent local mean sea level at any particular place. See also NOAA Web site: http://www.ngs.noaa.gov/faq. shtml\#WhatVD29VD88, and North American Vertical Datum of 1988

North American Datum of 1983 (NAD 83) The horizontal control datum for the United States, Canada, Mexico, and Central America that is based on the adjustment of 250,000 points including 600 satellite Doppler stations that constrain the system to a geocentric origin. NAD 83 has been officially adopted as the legal horizontal datum for the United States by the Federal government.

\section{$\mathbf{P}$}

parameter code A five-digit number used in the USGS computerized data system, National Water Information System, to uniquely identify a specific constituent or property. particle size The diameter, in millimeters, of a particle determined by sieve methods.

pesticides Chemical compounds used to control undesirable organisms. Major categories of pesticides include insecticides, miticides, fungicides, herbicides, and rodenticides.

pH of water The negative logarithm of the hydrogen-ion activity. Solutions with $\mathrm{pH}$ less than 7.0 standard units are termed "acidic," and solutions with a $\mathrm{pH}$ greater than 7.0 are termed "basic." Solutions with a pH of 7.0 are neutral. The presence and concentration of many chemical constituents commonly dissolved in water are affected, in part, by the hydrogen-ion activity of water. Biological processes including growth, distribution of organisms, and toxicity of the water to organisms also are affected, in part, by the hydrogen-ion activity of water.

polar pesticides Pesticides that are very water soluble and tend not to be adsorbed onto soil. These pesticides are also not very soluble in tissues and tend not to bioconcentrate in biota because of their low solubility.

precipitation Falling products of water-vapor condensation in the atmosphere, such as rain, snow, sleet, and hail.

\section{S}

sea level As used in this report, refers to one of the two commonly used national vertical datums (NGVD 1929 or NAVD 1988). See separate entries for definitions of these datums. See data-statement conversion in the table page for identification of the datum used in this report.

sediment Solid material that originates mostly from disintegrated rocks; when transported by, suspended in, or deposited from water, it is referred to as "fluvial sediment." Sediment includes chemical and biochemical precipitates and decomposed organic material, such as humus. The quantity, characteristics, and origin of sediment in streams are affected by environmental and land-use factors. Some major factors are topography, soil characteristics, land cover, and depth and intensity of precipitation.

specific conductance (conductivity) A measure of the capacity of water (or other media) to conduct an electrical current. It is expressed in microsiemens per centimeter at $25^{\circ} \mathrm{C}$. Specific electrical conductance is a function of the types and quantity of dissolved substances in water and can be used for 
approximating the dissolved solids content of the water. Commonly, the concentration of dissolved solids (in milligrams per liter) is from 55 to 75 percent of the specific conductance (in microsiemens). This relation is not constant from stream to stream and may vary in the same source with changes in the composition of the water.

stage The water-surface altitude in feet above the datum. If the water surface is below the datum, the stage is negative.

stage-discharge relation The relation between the water-surface altitude, called stage (gage height), and the volume of water flowing in a channel per unit time.

streamflow The discharge that occurs in a natural channel. Although the term "discharge" can be applied to the flow of a canal, the word "streamflow" uniquely describes the discharge in a naturally flowing stream. The term "streamflow" includes sources of water in addition to "runoff," and applies to discharge affected by diversion or regulation.

streamflow-duration percentiles Values on a scale of 100 indicating the percentage of time during which a flow is not exceeded. For example, the 90th percentile of river flow is greater than or equal to 90 percent of all recorded flow rates.

suspended As used in tables of chemical analyses, undissolved material in a water-sediment mixture. It is defined operationally as the material retained when environmental water samples are processed through a 0.45-micrometer filter.

suspended sediment The sediment that is maintained in suspension by the turbulent upward components of currents or that exists in suspension as a colloid. See also sediment

suspended-sediment concentration The velocity-weighted concentration of suspended sediment in the sampled zone (from the water surface to a point approximately 0.3 foot above the bed) expressed as milligrams of dry sediment per liter of water-sediment mixture. The analytical technique uses the mass of all the sediment and the net weight of the watersediment mixture in a sample to compute the suspended-sediment concentration. See also sediment and suspended sediment
T

total discharge The quantity of a given constituent, measured as dry mass or volume, that passes a stream cross section per unit of time. When referring to constituents other than water, this term needs to be qualified, such as "total sediment discharge," "total chloride discharge," and so on.

total recoverable The amount of a given constituent in a whole-water sample after a sample has been digested (usually using a dilute acid solution) that results in dissolution of only readily soluble substances. Complete dissolution of all particulate matter is not achieved by the digestion treatment, and thus the determination represents something less than the total amount (that is, less than 95 percent) of the constituent present in the dissolved and suspended phases of the sample. To achieve comparability of analytical data for wholewater samples, equivalent digestion procedures are required of all laboratories performing such analyses because different digestion procedures may produce different analytical results.

traceable thermometer A thermometer that has been found to conform to specific requirements of construction and accuracy.

turbidity An optical property of a liquid that causes light rays to be scattered and absorbed rather than transmitted in straight lines through water. Turbidity, which can make water appear cloudy or muddy, is caused by the presence of suspended and dissolved matter, such as clay, silt, finely divided organic matter, plankton and other microscopic organisms, organic acids, and dyes (ASTM International, 2003). The color of water, whether resulting from dissolved compounds or suspended particles, can affect a turbidity measurement. To ensure that USGS turbidity data can be understood and interpreted properly within the context of the instrument used and site conditions encountered, data from each instrument type are stored and reported in the USGS National Water Information System by using parameter codes and measurement reporting units that are specific to the instrument type, with specific instruments designated by the method code. Specific reporting units used in this report are as follows:

\section{NTUR (Nephelometric Turbidity}

Units) White or broad band [400-680 nm] light source, 90 degree detection angle, multiple detectors with ratio compensation. 
FNU (Formazin Nephelometric Units) Near infrared [780-900 nm] or monochrome light source, 90 degree detection angle, one detector.

FNMU (Formazin Nephelometric Multibeam

Units) Near infrared [780-900 nm] or monochrome light source, multiple light sources, detectors at 90 degrees and possibly other angles to each beam. For more information please see Anderson (2004).

\section{W}

water year In USGS reports dealing with surface-water supply, the 12-month period October 1 through September 30. The water year is designated by the calendar year in which it ends and which includes 9 of the 12 months. Thus, the year ending September 30, 2005, is called the "2005 water year." 